\title{
Bestimmung der prägenden Wesenszüge im Sport der griechisch-römischen Antike
}

\author{
Dissertation \\ zur Erlangung des sozialwissenschaftlichen Doktorgrades \\ der Sozialwissenschaftlichen Fakultät der \\ Georg-August-Universität Göttingen
}

vorgelegt von

Dietrich Ramba

aus Duderstadt

Göttingen 2014 
1. Gutachter: Prof. Dr. Arnd Krüger

2. Gutachter: Prof. Dr. Dr. hc. Wolfgang Decker

3. Gutachter: Prof. Dr. Wolfgang Krumbein 


\section{Inhaltsverzeichnis}

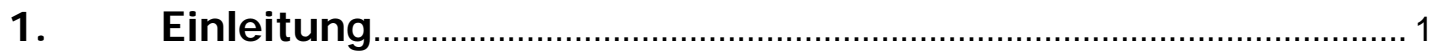

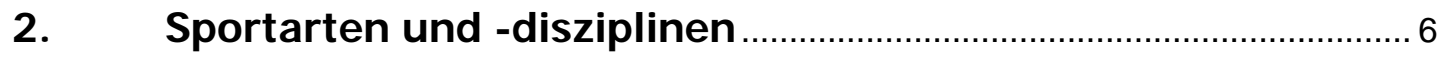

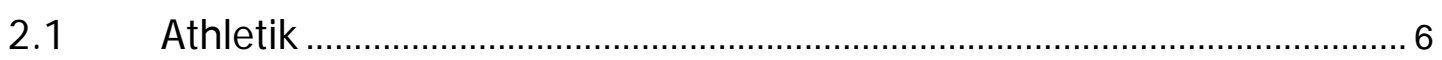

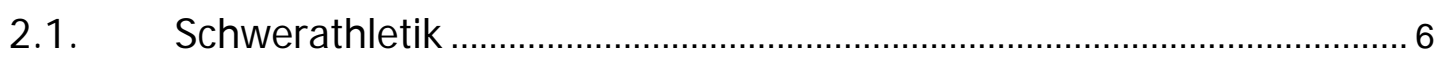

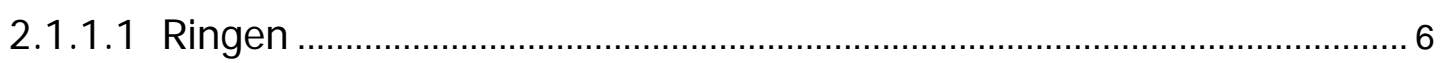

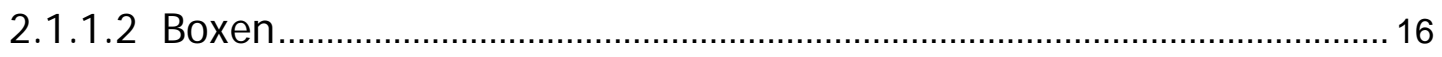

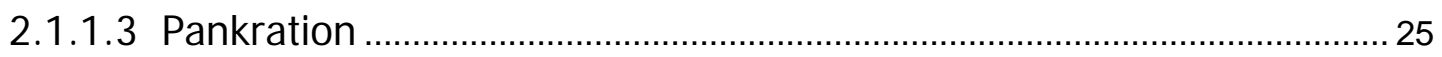

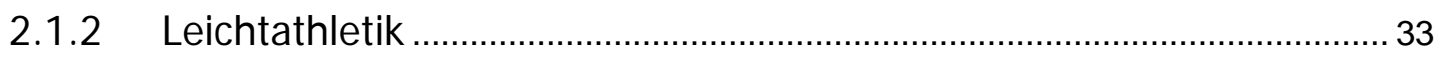

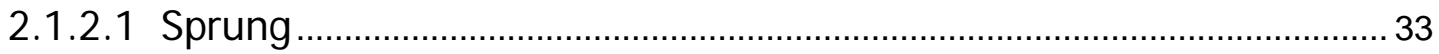

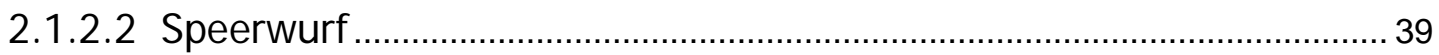

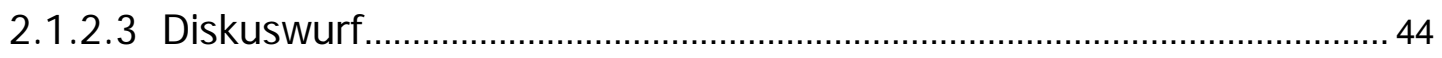

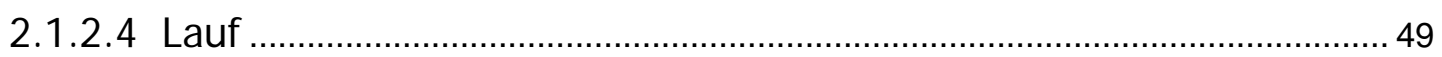

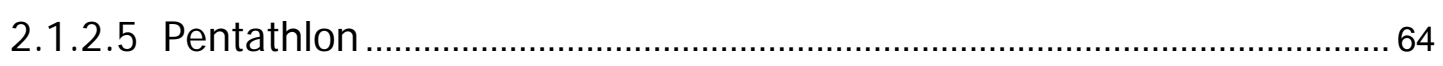

$2.2 \quad$ Ballspiele

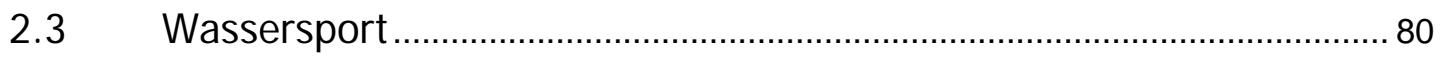

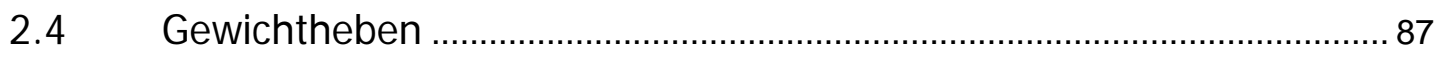

$2.5 \quad$ Bogenschießen ........................................................................................... 93

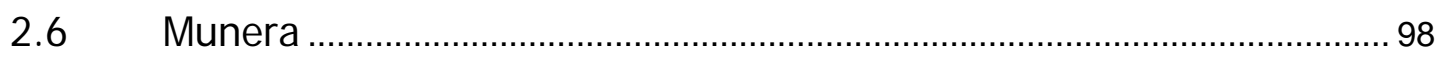

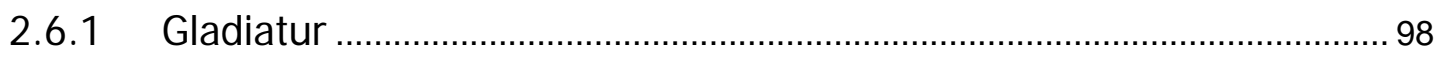

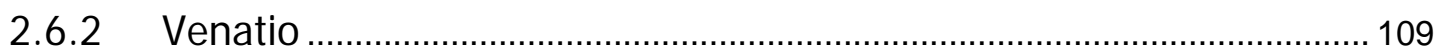

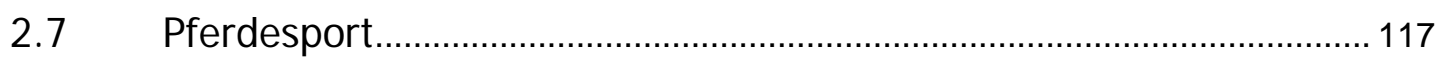

2.7.1 Hippische Wettkämpfe der Griechen....................................................117

2.7.2 Circensische Spiele der Römer ................................................................. 128

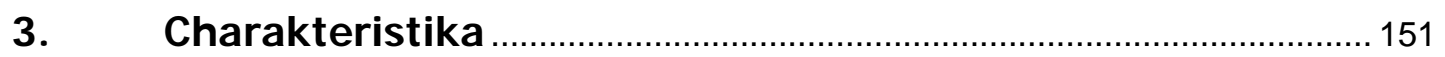

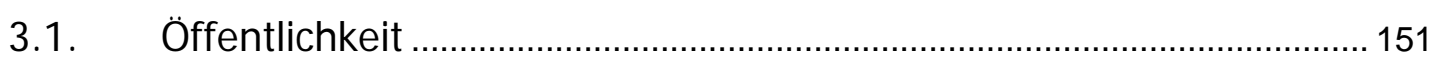

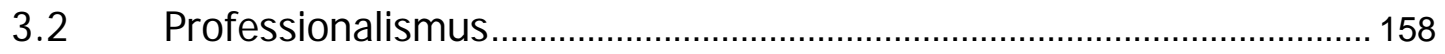

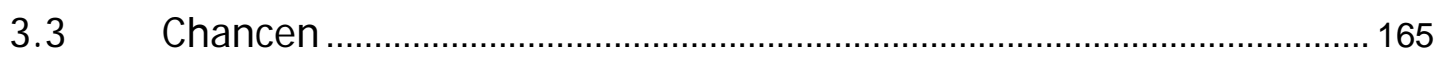

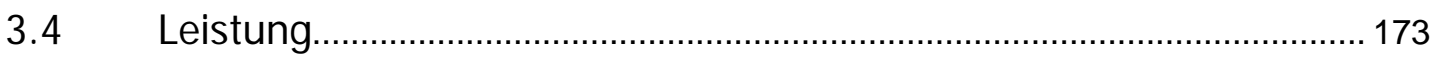




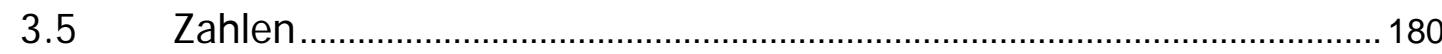

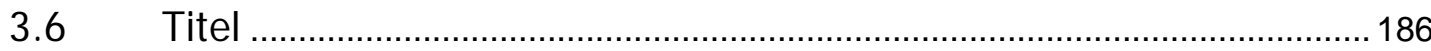

3.7 Rekorde

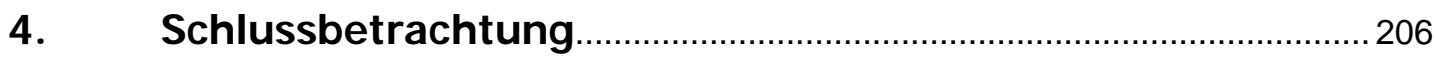

5. Abkürzungen und Literaturverzeichnis ….................................. 213

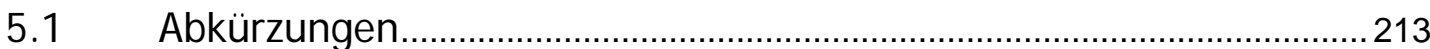

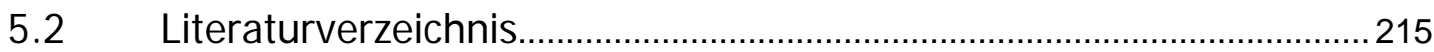




\section{Vorwort}

Die vorliegende Schrift ist die geringfügig überarbeitete Fassung meiner im Jahre 2012 von der Sozialwissenschaftlichen Fakultät der Georg-AugustUniversität Göttingen angenommenen Dissertation.

Angeregt hat die Untersuchung Herr Prof.Dr.Arnd Krüger,dem ich für seine überaus hilfreiche Kritik und fruchtbaren Ratschläge zu großem Dank verpflichtet bin.Auch seine Geduld wie auch seine Hartnäckigkeit waren wichtige Momente in meinem Schaffen.

Ferner möchte ich auch Herrn Prof. Dr. Dr .h.c.Wolfgang Decker danken, dessen Schriften zur Sportgeschichte nicht nur meine Kenntnisse über den antiken Sport erweitert haben, sondern auch meinen wissenschaftlichen Arbeitsstil maßgeblich geprägt haben.

Frau Katja Schnurr gilt mein besonderer Dank für die nicht nachlassende Mühe und Sorgfalt bei der Fertigstellung angesichts des schwierigen Manuskriptes v.a. aber für I hre eiserne Arbeitsdisziplin, die mir stets Vorbild und Antrieb war. 



\section{Einleitung}

Die vorliegende Studie dient der Herausarbeitung und Bestimmung der den Leistungs- und Wettkampfsport der griechisch-römischen Antike prägenden Charakteristika.

Die Untersuchung befasst sich mit dem Zeitraum von der ersten Austragung der Olympischen Spiele im Jahre 776 v. Chr. bis zu deren Verbot durch den christlichen Kaiser Theodosius I. im Jahre 393 n. Chr. Obwohl es in dieser Zeitspanne eine ganze Anzahl von Veränderungen im gesellschaftspolitischen als auch im sportlichen Bereich gegeben hatte, sahen wir vor allem durch die kanonisch wirkende Ausstrahlung der Olympischen Spiele, die auch noch die römischen Kaiser in ihren Bann zogen, den Sport der griechisch-römischen Antike aus sporthistorischer Sicht als eine Einheit.

Formal unterliegt die Arbeit einer Zweiteilung: Der erste Teil nennt und beschreibt die Sportarten und -disziplinen, die den Kanon des antiken Sports darstellen. ${ }^{1}$ Auf Basis dieser Untersuchung werden dann im zweiten Teil die Zuordnung und Bestimmung der erworbenen Kenntnisse hinsichtlich der prägenden Merkmale des Sports der griechisch-römischen Antike vorgenommen.

Den Anfang des ersten Teiles macht die Schwerathletik mit den Disziplinen Ringen, Boxen und Pankration (einem Kampfsport mit Elementen des Ringens und Boxens zusammen mit Beintechniken). Es folgt die Leichtathletik mit den technischen Disziplinen Weitsprung, Speer- und Diskuswurf, die gewöhnlich keine eigenständigen Konkurrenzen waren, sondern zumeist nur als Teildisziplinen des Pentathlons (Fünfkampf)durchgeführt wurden. Es schließt sich der Laufsport an, der ein breites Spektrum unterschiedlicher

II. WEI LER, Der Sport bei den Völkern der Alten Welt, Darmstadt 1988², W. DECKER, Sport in der griechischen Antike. Vom minoischen Wettkampf bis zu den Olympischen Spielen, Hildesheim $2012^{2}$; J .-P. THUI LLIER, Sport im antiken Rom, Darmstadt 1999. 
Disziplinen hinsichtlich Distanz, Austragungsmodus und Sportgerät bietet. Den Abschluss der Betrachtung zur Athletik bildet das Pentathlon, einem Mehrkampf, der sich aus den drei technischen Disziplinen (Weitsprung, Speer- und Diskuswurf), einem Kurzstreckenlauf und dem abschließenden Ringkampf zusammensetzt.

Es folgen Untersuchungen zu verschiedenen Ballspielen (Einzel-, Paar-, Kleingruppen- und Mannschaftsspiele), unterschiedlichen Formen des Wassersports (Schwimmen, Tauchen, Turmspringen, Segeln und Rudern), dem Gewichtheben und dem wettkampfmäßig betriebenen Bogenschießen (Ziel- bzw. Präzisions- und Weitenschießen).

Es schließen sich die munera, die sich aus den Gladiatorenkämpfen (/udi gladiatori) und den Tierkämpfen (venationes) zusammensetzen, an. Den Abschluss bildet der Pferdesport. Hierbei wird eine Trennung der hippischen Agone der Griechen von den circensischen Spielen der Römer vorgenommen, was vor allem aufgrund der Installation von Rennparteien im römischen Circuswesen Sinn machte.

In den Abschnitten zu den verschiedenen Sportarten und -disziplinen werden sowohl die Rahmenbedingungen wie auch die einzelnen für unsere Erhebung wichtigen Teilaspekte entsprechend dargestellt und ausgewertet, worunter z. B. Sportstätten, Sportgeräte, technische Ausführung, Kampfregeln, Schiedsrichter, Siegermittlung und Preise fallen.

Bei der Darstellung der Charakteristika im zweiten Teil der Arbeit ist bewusst auf eine weitere Unterteilung der Wesenszüge verzichtet worden, da so en bloc das einzelne Charakteristikum besser vermittelt bzw. intensiver wahrgenommen werden kann. J eder Abschnitt zu einem der Charakteristika ist in sich abgeschlossen; man wird jedoch erkennen, dass einige Wesenszüge ineinander- greifen bzw. einander bedingen (z. B. basieren einige Titel und Rekorde auf Zahlenwerten). Zu Beginn der Darstellung einiger Charakteristika ist diesen ein kurzer Exkurs über denselben Wesenszug in außersportlichen Bereichen (z. B. Politik, Militär, Wirtschaft, 
Kunst und Privatleben) vorangestellt, um die enge Verknüpfung von Gesellschaft und Sport erkennen zu lassen.

Im zweiten Teil der Arbeit beschäftigen wir uns zunächst mit den sportlichen Veranstaltungen als markantem Teil des öffentlichen Lebens der griechischrömischen Antike.

Anschließend wird hinterfragt, ob die leistungs- und wettkampfsportlichen Aktivitäten der Menschen zur damaligen Zeit einer beruflichen Tätigkeit gleichgestellt waren.

Es folgt die Auseinandersetzung mit der Frage der Chancen und Chancengleichheit im sportlichen Bereich in Anbetracht der sonstigen gesellschaftlichen Situation.

Auch liegt die Vermutung nahe, da die antike Gesellschaft sehr leistungsorientiert war, dass der Aspekt der Leistung und Leistungsmessung besonders stark im sportlichen Bereich zum Ausdruck kommt.

Danach wird ermittelt, welche Rolle Zahlen bei der Dokumentation sportlicher Leistungen spielten.

In Anlehnung an die auf Titel aufgebaute gesellschaftliche Hierarchie war zu klären, ob es im Sport ebenfalls zur Vergabe von Titeln kam und welche Bedeutung diese für den Titelträger mit sich brachte.

Den Abschluss der Arbeit bildet die Untersuchung hinsichtlich des Strebens nach Rekorden.

Anhand dieser Charakteristika soll zudem untersucht werden, inwieweit die von den Menschen der griechisch-römischen Antike betriebenen Aktivitäten bereits als Sport zu sehen sind, denn schließlich existiert die Vorstellung, Sport sei erst im 19. Jahrhundert in England entstanden. ${ }^{2}$

\footnotetext{
${ }^{2}$ Diese These ist v.a.von H.EICHBERG in zahlreichen Schriften vertreten worden und von einigen Kollegen unreflektiert übernommen worden
} 
Zur Erschließung unserer Thematik konnte aus einem sehr umfangreichen, Quellen- und Dokumentationsmaterial geschöpft werden, das aus einer Anzahl von unterschiedlichen Wissenschaften gespeist wurde: ${ }^{3}$

Hinsichtlich der Darstellung zur gesellschaftspolitischen Bedeutung des Sports in der greichisch-römischen Antike sind es vor allem Werke der Prosa ${ }^{4}$ und im Besonderen Schriften von Annalisten und Biografen, die unser Bild prägen.

Herausragende Bedeutung - in Verbindung mit der Epinikien ${ }^{5}$ - hat für uns die Epigrafik ${ }^{6}$. Neben ihrem Beitrag zur Prosopographie ${ }^{7}$ des antiken Sports sind es vor allem ihre detailreichen Informationen zu den Leistungen und Erfolgen der anitken Wettkampf- und Spitzensportler. ${ }^{8}$

Anschauliche Zeugnisse über die so zahlreich über den ganzen Mittelmeerraum verstreut gelegenen Übungs- und Wettkampfstätten liefern uns archäologische Ausgrabungsberichte. ${ }^{9}$

Allen voran waren es aber Reliefs, Mosaike, Vasen und Gegenstände der Kleinkunst, die uns dienliche Hinweise bezüglich der Sportgeräte, Technik, Regelwerk, Kampfrichterwesen, Siegermittlung, Preise u. v. m. lieferten.

\footnotetext{
3 J. JÜTHNER, Die athletischen Leibesübungen der Griechen, Bd. 1, Wien 1965, 21-41; I. WEILER, Sport, 277-288; DERS. (Hrsg.), Quellendokumentation zur Gymnastik und Agonistik im Altertum, 7 Bde., Wien u. a., 1991-2002

${ }^{4}$ H. GROOT, Zur Bedeutung der öffentlichen Spiele bei Tacitus, Sueton und Cassius Dio: Überlegungen zur Selbstbeschreibung der römischen Gesellschaft, Berlin u. a. 2008.
}

${ }^{5}$ A. P. BURNETT, Odes for Victorious Athletes, Baltimore 2010.

${ }^{6}$ M. LÄMMER, Die Bedeutung epigraphischer Zeugnisse für die Geschichte der griechischen Gymnastik und Agonistik, Köln 1968.

7 L. MORETTI, Olympionikai, i vincitori negli antichi agoni olimpici, Rom 1957; D. KYLE, Athletics in Ancient Athens, Leiden 1987; A. NEUMANN-HARTMANN, Prosopographie zu den Epinikien von Pindar und Bakchylides, in: Nikephoros 21, 2008, 81-131.

${ }^{8}$ L. MORETTI, Iscrizioni agonistiche greche, Rom 1953, J. EBERT, Griechische Epigramme auf Sieger an gymnischen und hippischen Agonen, Berlin 1972.

${ }^{9}$ A. HÖNLE/A. HENZE, Römische Amphitheater und Stadien, Zürich 1981; J. H. HUMPHREY, Roman Circuses: Arenas of Chariot Racing, London 1986, K. E. WELCH, The Roman Amphitheatre. From the Origions to the Colosseum, Cambridge 2007 
Zur Aufklärung technischer Abläufe und der Deutung inschriftlich überlieferter Leistungen halfen sportwissenschaftliche Versuche (z. B. beim Weitsprung). ${ }^{10}$

Die experimentelle Archäologie wiederum lieferte neue Einsichten in die Wettkampftechnik und -taktik der einzelnen Gladiatorengattungen, was Rückschlüsse auf das Kampfgeschehen zulässt und die zahlenmäßig erfassten Leistungen der Kämpfer erklärt. ${ }^{11}$

Forschungsergebnisse von Forensikern und Anthropologen ${ }^{12}$ erweiterten unsere Erkenntnisse über die Lebensweise (z. B. Ernährung) und das Training (z. B. Physis) bis zur Todesursache (z. B. tödliche Verletzunge durch Dreizack des Gegners) antiker SpitzensportlerBei aller Gewichtung auf die Darstellung mittels der Originalquellen bzw. -begriffe haben wir uns nicht gescheut, moderne Termini, die in wissenschaftlichen Arbeiten verwendet wurde, in unseren Beitrag aufzunehmen. So wurden auch gezielt Begriffe wie Grand Slam $^{13}$, Sportartikelindustrie ${ }^{14}$ oder Medienspezialist ${ }^{15}$ aufgegriffen, was zum einen die Sachverhalte anschaulicher macht und gleichzeitig eine Brücke vom antiken zum modernen Sport schlägt ${ }^{16}$.

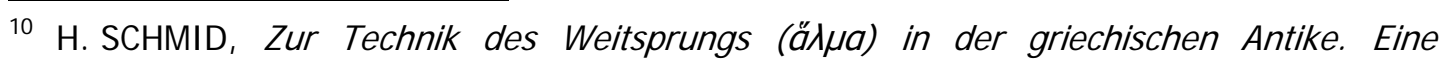
Neubewertung literarischer und bildlicher Quellen unter Berücksichtigung biomechanischer Bewegungsanalysen und volkskundlicher Tradition, Mainz 1997.

11 Eine große Hilfe zur Klärung des Ablaufs von Gladiatorenkämpfen brachten die Ergebnisse der experimentellen Archäologie. Vgl. M. JUNKELMANN, Das Spiel mit dem Tod. So kämpften Roms Gladiatoren, Mainz 2000, bes. $129 \mathrm{ff}$. und $156 \mathrm{ff}$.

${ }^{12}$ K. GROSSSCHMIDT/F. KANZ (Hrsg.), Gladiatoren in Ephesos. Tod am Nachmittag. Katalog zur Ausstellung im Ephesos Museum Selçuk, Wien 2002.

${ }^{13}$ W. DECKER, Sport, 32.

${ }^{14}$ W. BEHRINGER, Kulturgeschichte des Sports: Vom antiken Olympia bis zur Gegenwart, München 2012, 46.

${ }^{15}$ S. MÜLLER, Das Volk der Athleten. Untersuchungen zur Ideologie und Kritik des Sports in der griechisch-römischen Antike, Trier 1995, 276.

${ }^{16}$ Th. KLEMMER, Vom Siegerkranz zur Goldmedaille. Das Phänomen „Olympia“ und seine neuzeitliche Rezeption. Eine kulturhistorische Untersuchung unter besonderer Berücksichtigung der Berliner Spiele von 1936 , Bonn 2012, 3 


\section{Sportarten und -disziplinen}

In unserer Abhandlung werden neben den Sportarten und -disziplinen, die das Programm der Agone bestimmen, auch andere Sportarten und disziplinen behandelt, die man aufgrund zahlreicher Kriterien dem Leistungsund Wettkampfsport zurechnen kann.

\section{$2.1 \quad$ Athletik}

Die Athletik wird in Schwer- und Leichtathletik unterteilt. Es folgt das Pentathlon, das sich aus Disziplinen der Schwer- und Leichtathletik zusammensetzt.

\subsection{Schwerathletik}

Die Schwerathletik unterliegt einer Dreiteilung in Ringen, Boxen und Pankration.

\subsubsection{Ringen}

Der Ringkampf ${ }^{1}$ wurde als Einzeldisziplin ${ }^{2}$ und als einzige schwerathletische Übung im Rahmen des Pentathlon durchgeführt ${ }^{3}$. Die Ringer wurden nicht in

\footnotetext{
${ }^{1}$ Über Sonderformen des Ringkampfes vgl. Athen. 13, 566 c; Eustath. 331, 37; Ael. var. hist. 11, 1. Die Römer rangen nach griechischem Vorbild; vgl. L. FRIEDLÄNDER, SG 2, 149 ff.; F. MEZÖ, Geschichte der Olympischen Spiele, München 1930, 80. Zum Ringkampf bei den Etruskern vgl. J.-P. THUILLIER, in: Stadion 7.2, 1981, 178 f.; DERS., Les jeux athlétiques dans la civilisation étrusque, Rom 1985, $269 \mathrm{ff}$.

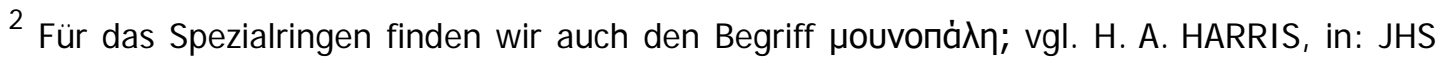
82, 1962, 22, n. 11; J. EBERT, in: WZ Halle 15.3, 1966, 381; anders L. MORETTI, IAG, 50, der darin eine Sonderform des Ringkampfes sieht, bei der es zum Sieg nur eines Niederwurfes des Gegners bedurfte.

3 Der Ringkampf im Pentathlon unterschied sich hinsichtlich des Regelwerks und der Siegerermittlung nicht von der Einzelkonkurrenz. Es wird jedoch aufgrund der unterschiedlichen Physis von Schwerathleten und Fünfkämpfern ein Unterschied in den
} 


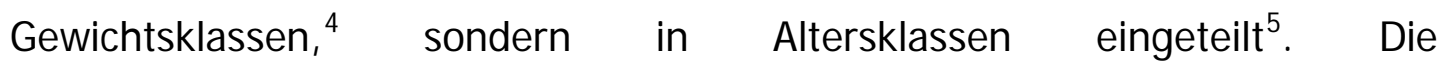
Zusammenstellung der Kampfpaare erfolgte mittels Los $^{6}$, und die Durchführung der Kämpfe bedurfte keiner Sportgeräte ${ }^{7}$. Die Wettkämpfe

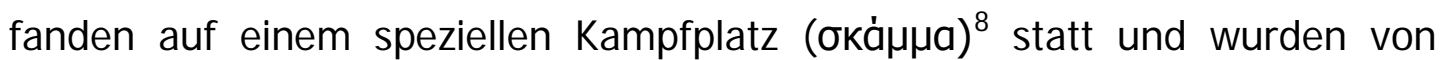
Kampfrichtern ${ }^{9}$ geleitet, die auf die Einhaltung der Kampfregeln ${ }^{10}$ achteten und über Sieg und Niederlage entschieden ${ }^{11}$. Der Sieg wurde dem Ringer zugesprochen, der seinen Gegner dreimal zu Boden gezwungen hatte ${ }^{12}$.

bevorzugten Techniken gegeben haben; vgl. W. RUDOLPH, Olympischer Kampfsport in der Antike, Berlin 1965, 57.

4 Paus. 6, 23, 2; vgl. I. WEILER, Sport , 172; W. DECKER, Sport, 70: „Da es nie eine Einteilung in Gewichtsklassen gegeben hat, was auch für den Faustkampf und das Pankration gilt.“

5 Vgl. R. PATRUCCO, Lo sport nella Grecia antica, Florenz 1972, 274; F. BREIN, Die Leibesübungen im alten Griechenland, GL 2, 111; N.-B. CROWTHER, The Age-Category of Boys at Olympia, in: Phoenix 42.4, 1988, 304 ff. (=Athletika 87 ff.).

${ }^{6}$ Paus. 6, 1, 2; Lukian. Hermot. 40; vgl. E. N. GARDINER, Wrestling I, in: JHS 25, 1905, 16; L. ROBERT, in: Hellenica 7, 1949, 107 f.; M. I. FINLEY/H. W. PLEKET, Die Olympischen Spiele der Antike, Tübingen 1976, 81; I. WEI LER, Sport, 172 f.

${ }^{7}$ Zur Bekleidung der Ringkämpfer mit dem Schurz vgl. R. PATRUCCO, Sport, 272; F. BREIN, GL 2, 110. Zum Einölen der Athleten vgl. Ch. ULF, in: Stadion 5, 1979, 220 ff. Zur Verwendung eines Kopfschutzes im Training vgl. R. PATRUCCO, Sport, 273; J. SWADDLING, The Ancient Olympic Games, London 19804, $58 \mathrm{f}$.

${ }^{8}$ Vgl. E. N. GARDINER, in: JHS 25, 1905, 18; DERS., GASF, 376; J. JÜTHNER, in: RE 3 A 1 (1927), s. v. ¿Kó $\mu \mu a, 436$ f.; DERS., in: RE 18, 3 (1949), s. v. Pale, 85; H. A. HARRIS, GAA, 104; R. PATRUCCO, Sport, 272; F. BREIN, GL 2, 112 J. SWADDLING, Games, 57.

${ }^{9}$ Bezeichnend für die relativ wenigen Darstellungen von Kampfrichtern bei Ringkämpfen ist, dass sie nie in das Kampfgeschehen eingreifen; vgl. aber auch Ael. var. hist. 2, 6.

${ }^{10}$ Plat. leg. 8, 33 e; Ael. var. hist. 11,1; vgl. J. JÜTHNER, in: RE 18,3 (1949), s. v. Pale, 83; W. RUDOLPH, Kampfsport, 37 f.; M. POLIAKOFF, Studies in the Terminology of the Greek Combat Sports, Königstein 1982, 7 ff. Vgl. auch Suet. Ner. 53.

11 In den anderen schwerathletischen Disziplinen war gewöhnlich der subjektive Verzicht eines der Kontrahenten für den Kampfentscheid ausschlaggebend.

12 Philostr. gymn. 11; Bekker, Anecd. 1, 114, 22; Sen. benef. 5, 9. H. A. HARRIS, GAA, 102: „A match was for the best of three falls." Vgl. auch L. ROBERT, Les épigrammes satiriques 
Besonderes Interesse galt neben der Zahl der Siege v. a. der Art und Weise, in der die Siege oder Siegesserien errungen wurden. Einige Athleten betonten ihre gewaltige Körperkraft ${ }^{13}$, die ihnen den Sieg gebracht hatte, andere führten ihre Erfolge auf die glänzende Technik zurück ${ }^{14}$, und wieder andere rühmten sich beider Qualitäten ${ }^{15}$. Besonders vermerkt wurden auch Siege, die durch eine kluge Taktik ${ }^{16}$ oder die Anwendung einer Spezialtechnik ${ }^{17}$ gelangen, wobei das Fingerbrechen ${ }^{18}$ nicht löblich war ${ }^{19}$. Der

de Lucillius sur les athlètes: Parodie et réalité, Genf 1968, 253; R. PATRUCCO, Sport, 298. Zu sporttechnischen Begriffen bezüglich des Ringkampfes vgl. H. A. HARRIS, GAA, 207, n. 59; R. PATRUCCO, Sport, 275 ff.; M. POLIAKOFF, Terminology, 155 ff.; W. DECKER, Sport, 70.

13 Anth. Pal. 16, 1; SEG 24, 387; vgl.J. EBERT, Griechische Epigramme auf Sieger an gymnischen und hippischen Agonen, Berlin 1972, Nr. 25; Zu yopyòs in Athletenepigrammen vgl. J. BINGEN, in: BCH 77, 1953, 639.

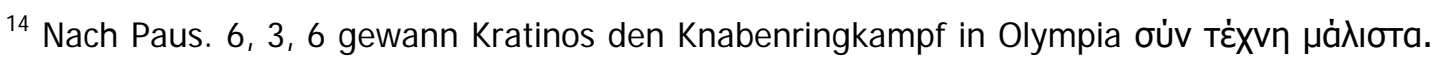

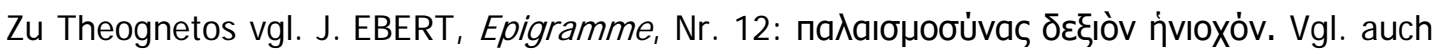
ebd. Nr. 34. Vgl. ferner F. MEZÖ, Geschichte, 83; W. RUDOLPH, Kampfsport, 59; J. SWADDLING, Games, 57; W. DECKER, Sport, 71.

15 SEG 13, 516; L. MORETTI, IAG, Nr. 48; J. EBERT, Epigramme, Nr. 73. Die Vorstellungen vom idealen Athleten waren in den einzelnen Landschaften recht verschieden und führten zu unterschiedlicher Wertschätzung von kraftvollem Stil und ausgefeilter Technik. Vgl. J. EBERT, Epigramme, 114. Jedoch allgemein verbindlich Thuk. 2, 87, 4.

${ }^{16}$ Diog. Laert. 8, 1, 12: Der schmächtige Eurymenes (Ol. Sieger 472 v. Chr.) besiegte viele mächtige Gegner dank der oopia seines Trainers Pythagoras. Ähnlich Pind. Ol. 8, 54; Nem. 4, 6; vgl. M. WOLOCH, Athenian Trainers in the Aeginetan Odes of Pindar and Bacchylides, in: CW 56, 1963, 102 ff.; K. KRAMER, Studien zur griechischen Agonistik nach den Epinikien des Pindar, Köln 1970, 70 ff.

17 Paus. 6, 4, 3; Suda s. v. tpaxndiłwv. Vgl. L. MORETTI, Olympionikai, i vincitori negli antichi agoni olimpici, Rom 1957, Nr. 607; H. BUHMANN, Der Sieg in Olympia und in den anderen panhellenischen Spielen, München 1975², 34. Philostr. gymn. 41 berichtet, dass der Ringer Mus mit der einst verletzten linken Seite nicht zu stoppende Angriffe ausführte.

${ }^{18}$ L. MOREITI, in: RFIC 34, 1956, 58 f.; S. G. MILLER, Arete, Chicago 1979, 20.

${ }^{19}$ Paus. 6, 4, 3 sagt, dass Leontiskos es nicht verstanden habe, die Gegner durch Würfe zu bezwingen und nur durch Fingerbrechen gesiegt habe. H. A. HARRIS, GAA, 103, glaubt, dass das Fingerbrechen daraufhin verboten wurde, da man später von solch einer Form des 
Kampfentscheid durch den Tod eines der Kontrahenten war eher beklagenswert, ${ }^{20}$ hatte aber keine Rechtsfolgen. ${ }^{21}$

Allein die Zulassung zum Wettkampf wurde schon als Erfolg gefeiert ${ }^{22}$, eine überzeugende Teilnahme ${ }^{23}$ oder sogar das Erreichen des Finales führte man stolz $\mathrm{an}^{24}$, und ein Unentschieden im Endkampf konnte einem Erfolg gleichgestellt sein. ${ }^{25}$ Die Wertschätzung eines Finalsieges wurde gemindert, wenn man im Laufe der Konkurrenz durch ein Freilos begünstigt worden war ( $\left.\varepsilon^{\prime} \varphi \varepsilon \delta \rho \circ \varsigma\right) .{ }^{26}$ Hatte man alle Runden mitgerungen $(a ́ v \varepsilon ́ \varphi \varepsilon \delta \rho \circ \zeta)^{27}$, dazu viele

Sieges nichts mehr hört. I. WEI LER, Sport, 173, spricht von dem „rühmenden (sic) Beinamen ,Fingerbrecher' “.

20 Syll.3 274; L. MORETTI, IAG, Nr. 29; vgl. R. BROPHY/M. BROPHY, Deaths in the PanHellenic Games, //: All Combative Sports, in: AJPh 106, 1985, 172 ff.

${ }^{21} \mathrm{~J}$. EBERT, Epigramme, 144: „Von unvorsätzlicher Tötung beim Wettkampf ist in der Literatur mehrfach die Rede; sie wurde strafrechtlich nicht verfolgt.“

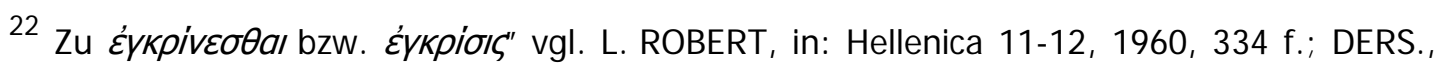
Lucillius, 186. Vgl. auch Paus. 6, 14, 1, wo der Athener Aristophon wegen seines geringen Alters nicht zum Kampf zugelassen wurde.

23 Vgl. z. B. E. LEGRAND/J . CHAMONARD, Inscriptions de Phrygie l, in: BCH 17, 1893, 256, Nr. 35: „...á

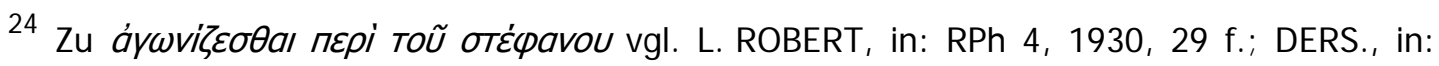
Hellenica 2, 1945, 71; 11-12, 1953-1954, 334; DERS., Études anatoliennes, Amsterdam $1970^{2}, 140$.

25 IG 14, 739; SEG 2, 745; 3, 335; vgl. L. MORETTI, IAG, Nr. 77; vgl. J. JÜTHNER, in: WS 24, 1902, 287 f.; L. ROBERT, in: RPh 4, 1930, 28; DERS., in: Hellenica 2, 1946, 6; 11-12, 1960, 356 f.; DERS., in: RA 1978, 282 f.; R. PATRUCCO, Sport, 299, n. 9; R. MERKELBACH, in: ZPE 11, 1973, 129.

26 Vgl. A. E. J. HOLWERDA, in: AZ 38, 1881, 171 f.; F. MIE, Quaestiones agonisticae imprimis ad Olympia pertinentes, Diss. Rostock 1888, 39 f.; J. JÜTHNER, in: RE 5, 2 (1905), S. v. ह̋ $\varphi \varepsilon \delta \rho o s, 2747$ f.; E. N. GARDINER, in: JHS 25, 1905, 17 f., N. YALOURIS (Hrsg.), The Olympic Games, Athen 1972, $122 \mathrm{f}$.

${ }^{27} \mathrm{Vgl}$. L. MORETTI, IAG, $171 \mathrm{ff} ., \mathrm{S}$. BRUNET, Winning the Olympics without Taking a Fall, Getting Caught in a Waistlock or Sitting out a Round, in: ZPE 172, 2010, 115. 
Gegner bezwungen ${ }^{28}$ oder auf dem Weg zum Finalsieg einen durch das Losglück begünstigten Ringer überwunden ${ }^{29}$, so wertete dies den Sieg auf. Glänzende Siege wurden mit dem Zusatz von $\varepsilon^{\prime} \delta \delta \dot{\xi} \xi \omega \varsigma$ hervorgehoben ${ }^{30}$, so wie man Siege in Wettkämpfen, die zu einem feierlichen Empfang in der

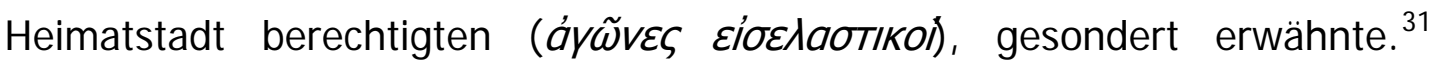

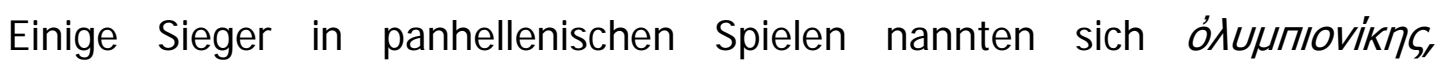

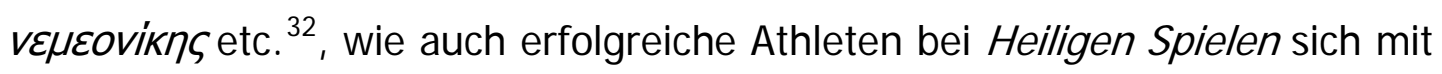

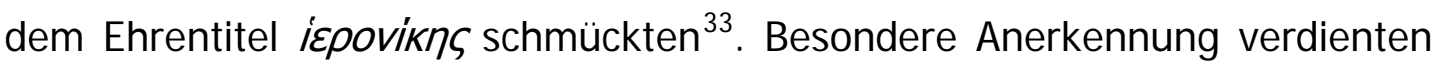
Ringer, die einen Kampf bereits nach drei von fünf möglichen Durchgängen gewonnen hatten ( Laufe der letztlich siegreich abgeschlossenen Konkurrenz man nicht zu Fall gebracht worden war (ánт $\left.\omega^{\prime}\right)^{35}$. In die gleiche Richtung zielen die Aussagen,

28 Vgl. W. PEEK, Zwei agonistische Gedichte, in: Hermes 77, 1942, 209, (3 Gegner bezwungen); IvO 164; L. MORETTI, IAG, Nr. 20; Pind. Ol. 8, 66 ff.; Pyth. 8, 81 f. (4 Gegner

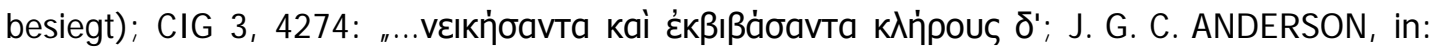

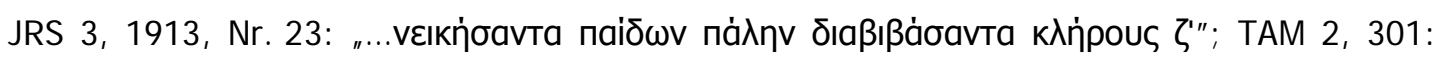

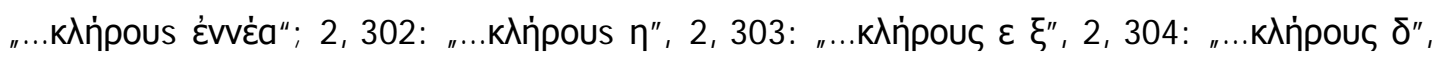
vgl. L. ROBERT, in: Hellenica 7, 1949, 108; DERS., Lucillius, 251 n. 2.

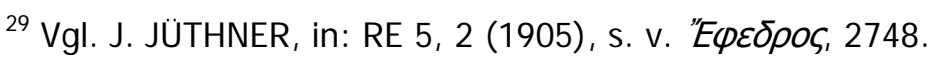

30 CIG 3503; 3951, IGR 4, 1268; TAM 2, 944; 1206; MAMA 6, 80; vgl. L. ROBERT, in: Hellenica 11-12, 1960, 351 f. u. 365 f.; DERS., Lucillius, 186 f.; R. MERKELBACH, in: ZPE 18, 2, 1975, 125.

${ }^{31}$ Vgl. L. ROBERT, in: REG 74, 1961, 149 f.; DERS., in: RPh. 61, 1967, 18 ff.; DERS., Étud. anat, 119 n. 3; P. HERRMANN, Eine Kaiserurkunde aus der Zeit Mark Aurels aus Milet, in: MDAI (I) 25, 1975, 156; H. W. PLEKET, Zur Soziologie des antiken Sports, in: MNIR 36, 1974, 71 u. 85, n. 140; DERS., in: Stadion .1. 1, 1975, 62 f.; D. C. YOUNG, The Olypmic Myth of Greek Amateur Athletics, Chicago 1985, 54.

32 Vgl. L. ROBERT, in: RA 1934, 54 f. Zu einer Wertigkeit der Siege gemäß der Festspielregion bzw. -orte vgl. L. MORETTI, in: RFIC 82, 1954, $276 \mathrm{ff}$.

${ }^{33}$ Vgl. R. MERKELBACH, in: ZPE 18, 2, 1975, 128.

34 Philostr. gymn. 11; Anth. Pal. 5, 588; Sen. benef. 5, 3; Bekker, Anecd 1, 114, 22; vgl. R. PATRUCCO, Sport, 298; J. SWADDLING, Games, 57.

35 IVO 164; Pind. Ol. 9 29; Bakchyl. 10, 22; Anth. Pal. 9, 588; TAM 2, 301; CIG 5924; L. MORETTI, IAG, Nr. 20; 47 f.; J. EBERT, Epigramme, Nr. 32; 72; vgl. R. KNAB, Die 
dass man nicht auf die Knie gezwungen worden war $^{36}$ - was Milon von Kroton bei all seinen Olympischen Spielen gelang ${ }^{37}$ - oder die Schultern nicht den Boden berührt hatten ${ }^{38}$. Einige Athleten priesen sich, nicht nur unbesiegt geblieben, sondern nicht einmal in eine bedrohliche Kampfsituation geraten

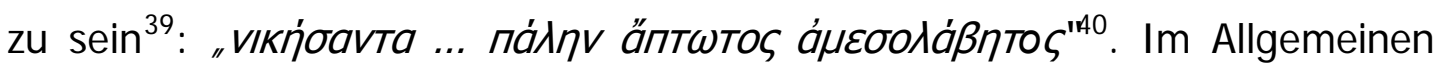
wurde mit äıвıпто $\varsigma^{41}$ angezeigt, dass man unbesiegt war oder eine Siegesserie in dieser Weise vollbracht hatte ${ }^{42}$. Die wohl anerkannteste Form des Erfolges war der kampflose Sieg, der mit ákoviti angegeben wurde ${ }^{43}$. Meistens war es die Anerkennung der Überlegenheit eines der Konkurrenten, die zu dieser Form des Sieges führte ${ }^{44}$. Eines solchen kampflos zugesprochenen Sieges rühmten sich die Athleten auch durch den Vermerk

Periodoniken, Diss. Gießen 1934, 15; J. JÜTHNER, in: Glotta 29, 1941, 75; L. ROBERT, in: Hellenica 7, 1949, 108; DERS., in: RPh 41, 1967, 23; DERS., Lucillius, 249 ff.; G. DUNST, in: ZPE 3, 1968, 139 ff.; R. MERKELBACH, in: ZPE 11, 1973, 257 ff.; W. RUDOLPH, Kampfsport, 30 n. 3; F. BREIN, GL 2, 111.S.BRUNET,in:ZPE $172,2010,115$.

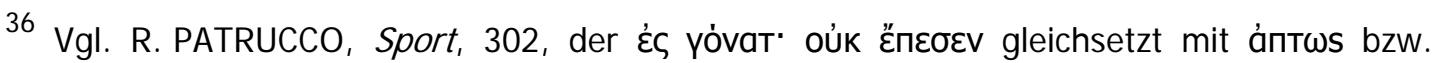

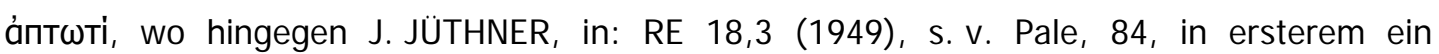
größeres Lob sieht.

${ }^{37}$ Anth. Pal. 16, 24; vgl. L. ROBERT, Lucillius, 252; N. YALOURIS, Games, $266 \mathrm{f}$.

38 IG, 14, 1107; Anth. Pal. 9, 588; 16, 25; J. EBERT, Epigramme, Nr. 67; vgl. R. KNAB, Periodoniken, 38; R. PATRUCCO, Sport, 302.

39 Vgl. R. KNAB, Periodoniken, 14; L. ROBERT, in: AC 37, 1968, 409 f.; R. MERKELBACH, Der griechische Wortschatz und die Christen, in: ZPE 18, 1975, 146 ff.; M. POLIAKOFF, in: ZPE 44, 1981, 80 n. 12; DERS., Terminology, $42 \mathrm{f}$.

40 Jul. Afric. Ol. 98; TAM 2, 741; 1206 f.; $v g l . S . B R U N E T$, in:ZPE 172 ,2010, 115.

${ }^{41}$ Jul. Afric. Ol. 118; IG 14, 1102; $1104 \mathrm{f}$.

42 Vgl. R. KNAB, Periodoniken, 13; L. ROBERT, in: Hellenica 11-12, 1960, 332 ff.; J. EBERT, Epigramme, 239; vgl. ebd. 203 allgemein zu $\lambda \varepsilon i n \varepsilon \sigma \theta a \mathrm{a}=$ unterliegen.

43 Jul. Afric. Ol. 147; Anth. Pal. 11, 316; Suda, s. v. ákovití.

44 Vgl. E. REISCH, in: RE 1 (1894), s. v. AKoviti, 1178; F. MEZÖ, Geschichte, 81; J. JÜTHNER, in: Glotta 29, 1941, 76 f.; L. ROBERT, in: Hellenica 7, 1949, 110; DERS., Al 1, 130; DERS., in: REG 79, 1966, 169; DERS., Lucillius, 247 f.; J. EBERT, Epigramme, 53; R. PATRUCCO, Sport, 298 f.; H. BUHMANN, Sieg, 18 n. 6; N. YALOURIS, Games, 123 f.; J. SWADDLING, Games, 58. 


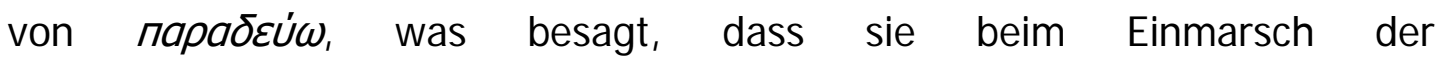
Wettkampfteilnehmer in das Stadion die einzigen Athleten waren, die in der Disziplin und Altersklasse antraten ${ }^{45}$. Von anderen Siegern wird ähnliches berichtet, dass nämlich aufgrund ihrer Eintragung in die Meldeliste die gesamte Konkurrenz ihre Meldung zum Wettkampf zurücknahm und sie dadurch den Sieg zugesprochen bekamen ${ }^{46}$. Die Überlegenheit des Ringers

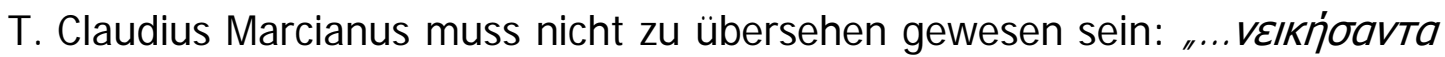

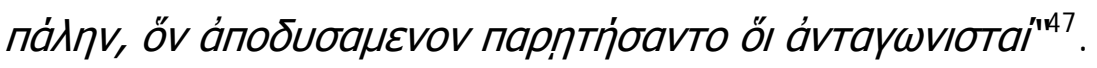

Im Bereich der Schwerathletik waren zahlreiche Athleten in allen drei Disziplinen aktiv und erfolgreich ${ }^{48}$, so dass Mehrfachsiege an einem Tag in verschiedenen Disziplinen ${ }^{49}$ und/oder in verschiedenen Altersklassen ${ }^{50}$ nicht

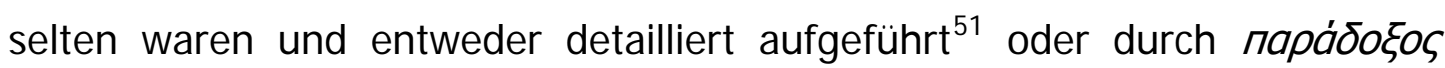

${ }^{45}$ Vgl. L. ROBERT, in: Hellenica 5, 1948,75 f.; DERS., in: REG 81, 1968, 147; G. E. BEAN, in:

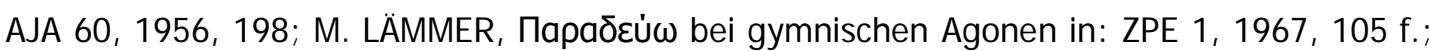
R. MERKELBACH, in: ZPE 18.2, 1975, 120.

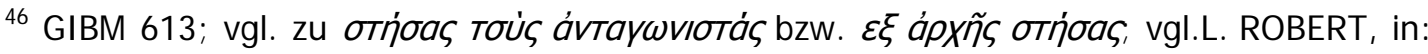
Hellenica 7, 1949, $110 \mathrm{f}$.

${ }^{47}$ Vgl. W. CRÖNERT, in: JÖAl 12, 1909, 151; J. G. C. ANDERSON, in: JRS 3, 1913, 287 Nr. 12; J. JÜTHNER, in: Glotta 29, 1941, 77; L. ROBERT, in: Hellenica 7, 1949, 110, der noch darauf hinweist, dass die Aufgabe der Athleten vor dem Losen erfolgt sein muss, da die Teilnehmer zur Auslosung entkleidet erschienen. Vgl. auch M. I. FINLEY /H. W. PLEKET, Spiele, 116.

48 Die Zahl der Siege im Ringkampf ist dabei gewöhnlich am niedrigsten; vgl. L. MORETTI, $I A G, \mathrm{Nr} .40 ; 51$. Selten waren Schwerathleten in anderen Disziplinen erfolgreich; vgl. Paus. 5,9,1; 6,15,4 f.; J. EBERT, Epigramme, Nr. 1.

49 Paus, 6,15,3 ff.; Anth. Pal. 9,588; L. MORETTI, IAG, Nr. 40; 51; 62; 68; vgl. L. ROBERT, Lucillius, 250; R. PATRUCCO, Sport, 274; H. BUHMANN, Sieg, 11.

50 IGR 4,491; vgl. L. ROBERT, Étud. anat., 50. Vgl. auch Paus. 6,14,1, wo der Knabe Nikasylos von Rhodos den Männern zugewiesen wird und dort siegt.

51 Paus. 7, 23, 5; Anth. Pal. 9, 588; IG 4, 428; vgl. Th. KLEE, Zur Geschichte der gymnischen Agone an griechischen Festen, Leipzig/Berlin 1918, 54; R. KNAB, Periodoniken, 37; L. ROBERT, in: Hellenica 11-12, 1960, 348 ff.; DERS., Lucillius, 192; R. PATRUCCO, Sport, 274; H. BUHMANN, Sieg, 41. 
bzw. парабоқоvikns vermerkt wurden ${ }^{52}$. Athleten, die in Olympia den Ringkampf und das Pankration an einem Tage gewannen, die so genannten Herakles-Sieger, gehören zu dieser Kategorie ${ }^{53}$. Siegesserien bei einem Fest oder verschiedenen Veranstaltungen in Folge wurden oft mit катá тó $\dot{\varepsilon} \xi \tilde{\eta} \varsigma$ angegeben $^{54}$. Viele Athleten pflegten die errungenen Siege - z. T. mit Hervorhebung besonderer Erfolge ${ }^{55}$ - chronologisch oder ihrer Wertigkeit nach aufzulisten ${ }^{56}$. Dabei wurden (fast) alle Siege mit Angabe des Festes bzw. des Austragungsortes und der Altersklasse, in der man gewonnen hatte, angegeben ${ }^{57}$. Die Zahl der Siege konnte auch in Bezug auf den

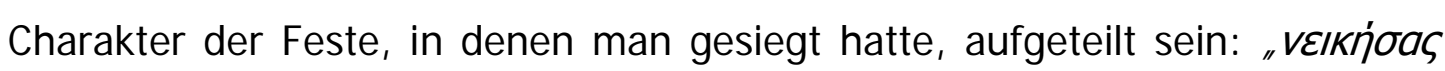

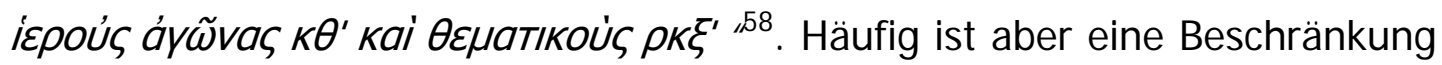
der detaillierten Angaben auf eine Reihe bedeutender Feste ${ }^{59}$ oder auf die Zyklus-Spiele ${ }^{60}$ festzustellen. Diesen Angaben konnte eine konkrete Zahl der

52 Vgl. L. ROBERT, in: RPh 4, 1930, 38 ff.; DERS., in: RA 1934, 53; R. MERKELBACH, in: ZPE 14, 1974, 91 ff.; H. BUHMANN, Sieg, 33, n. 2; Ch. HABICHT, Pausanias, München 1985, 81; vgl. auch L. MORETTI, IAG, 189.

53 Vgl. L. FRIEDLÄNDER, SG 2, 147 ff.; R. KNAB, Periodoniken, 6; C. A. Forbes, O। $a \varphi^{\prime}$ Hpakléous in Epictetus and Lucianus, in: AJ Ph 60, 1939, 473 f.; W. DECKER, Sport, 68.

54 Paus. 7, 27, 7; in: IGR 4, 1344; vgl. L. ROBERT, in: RPh 4, 1930, 42.

55 Dazu wurden gewöhnlich die oben und nachfolgend aufgeführten Epitheta verwandt. Zu yaũpos in Athletenepigrammen vgl. L. ROBERT, in: Hellenica 2, 1946, 139 ff.; zu áxıvĩ̌os in Athletenepigrammen vgl. L. ROBERT, in: Hellenica 11-12, 1960, 358.

${ }^{56}$ Vgl. z. B. Th. KLEE, Geschichte, 55.

${ }^{57}$ Vgl. z. B. L. MORETTI, IAG, Nr. 65; 68.

58 IG 14, 739; in: IGR 1, 444; vgl. L. ROBERT, in: RPh 4, 1930, 42 ff.; zu der in dieser Inschrift angegebenen erstaunlich hohen Zahl von 19 Unentschieden vgl. L. MORETTI, IAG,

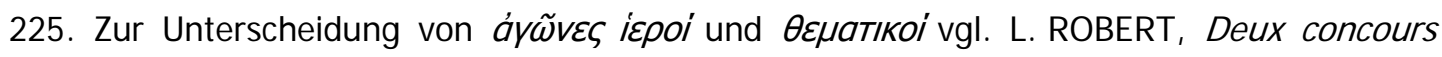
grecs à Rome, in: CRAl 1970, 6 ff.

${ }^{59}$ Vgl. P. ROUSSEL, in: BCH 31, 1907, 432 ff.; Th. KLEE, Geschichte, 56 f.; St. Dow, Hesperia

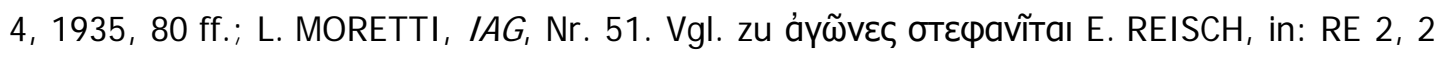

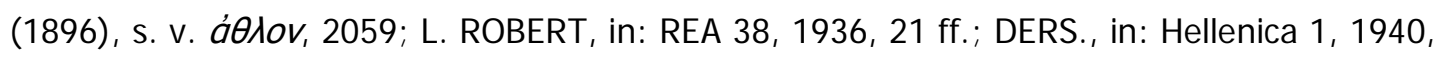
114 ff.; DERS., in: CRAl 1970, 6 ff.; J. EBERT, in: WZ Halle, 15, 3, 1966, 382; H. W. PLEKET, Games, Prizes, Athletes and Ideology, in: Stadion 1.1, 1975, $56 \mathrm{ff}$.

${ }^{60}$ Vgl. z. B. J. EBERT, Epigramme, Nr. 34; 36. 
gesamten Siege folgen, ${ }^{61}$ wie auch eine solch genaue Zahlenangabe im Zusammenhang mit den Erfolgen des Vaters ${ }^{62}$ oder des Geschlechts ${ }^{63}$ zu finden war oder auf den Trainer allein bezogen sein konnte. ${ }^{64}$ War keine genaue Angabe gemacht, so wurde versucht, die Zahl der Siege als

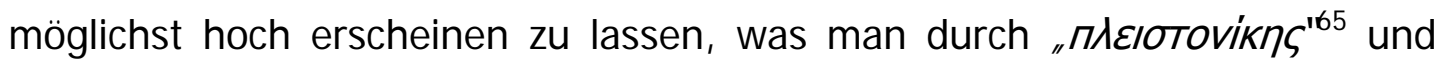

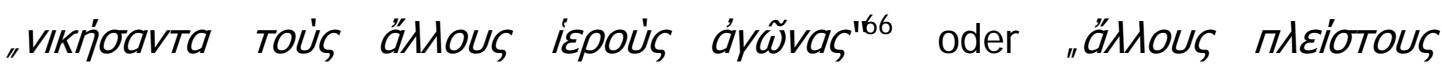

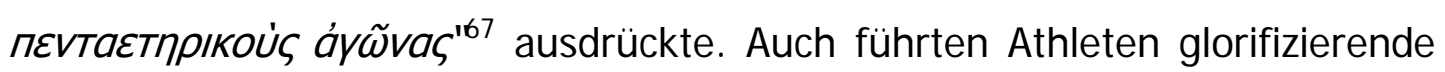
Schlusssätze in ihren Siegerepigrammen an, die das Ermessen ihrer

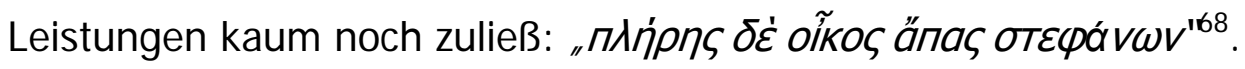

Als besonders erfolgreiche Ringer galten die Periodoniken ${ }^{69}$, jene Athleten, die bei den Olympien, Pythien, Isthmien ${ }^{70}$ und Nemeen gesiegt hatten, wobei es zusätzlichen Ruhm brachte, wenn man den Zyklus in dem kürzest

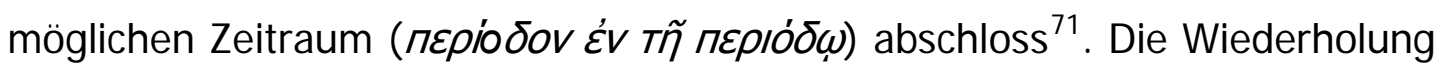

${ }^{61}$ Vgl. z. B. L. MORETTI, IAG, Nr. 21; J. EBERT, Epigramme, Nr. 37.

62 Paus. 3, 13, 9, vgl. auch L. MORETTI, IAG, Nr. 52; H. A. HARRIS, GAA, 123; J. EBERT, Epigramme, Nr. 74.

63 Pind. Ol. 8,65; Nem. 6,58 ff.; vgl. K. KRAMER, Studien, 90.

${ }^{64}$ Pind. Ol. 8, 65 f.; vgl. J. MI LLER, in: RE 15, 1 (1931), s. v. Melesias 1, 495.

65 IG 5, 1554; vgl. R. KNAB, Periodoniken, 14 n.41 ; L. ROBERT, in: RPh 4, 1930, 47; DERS., in: Hellenica 13, 1965, 140; L. MORETTI, IAG, 163; R. MERKELBACH, in: ZPE 14, 1974, 95 f. ${ }^{66}$ Vgl. Th. KLEE, Geschichte, 56 f.; R. KNAB, Periodoniken, 36; L. MORETTI, IAG, Nr 51.

67 IG 14, 747; in: IGR 1, 446; vgl. L. MORETTI, IAG, Nr. 68.

${ }^{68}$ Vgl. J. EBERT, Epigramme, Nr. 74; L. MORETTI, IAG, Nr. 52; vgl. auch Pind Nem. $10,45 \mathrm{f}$.

69 Vgl. R. KNAB, Periodoniken, 4 ff.; H. C. MONTGOMERY, in: RE 19,1 (1937), s. v. Periodonikai, 813 ff.; L. MORETTI, Note sugli antichi periodonikai, in: Athenaeum 32, 1954, 115 ff. Zur neuen Periodos der Kaiserzeit vgl. J. u. L. ROBERT, in: REG 67, 1954, 114 f.; K. KRAMER, Studien, 29; H. W. PLEKET, in: Stadion 1.1, 1975, 65 ff.; W. DECKER, Sport, 32. 70 Die Eleer waren von den isthmischen Spielen ausgeschlossen (Paus. 5, 2), so dass innen nicht die Möglichkeit gegeben war, Periodonike zu werden, vgl. R. KNAB, Periodoniken, 9; J. EBERT, Epigramme, 34.

${ }^{71}$ Vgl. L. ROBERT, in: RPh 4, 1930, 49; R. KNAB, Periodoniken, 9; L. MORETTI, IAG, 35. 
eines Zyklussieges gelang nicht einmal einem halben Dutzend von Ringern ${ }^{72}$, so dass die Leistung des berühmtesten Athleten der Antike ${ }^{73}$ nicht hoch genug eingeschätzt werden kann: Milon von Kroton war sechsfacher Periodonike im Ringkampf ${ }^{74}$.

Neben der feinen Unterteilung der Siege bzw. ihrer Wertigkeit, einer zahlenmäßigen Erfassung der Leistungen und häufig einer Kombination dieser Aspekte finden sich zahlreich die Angaben über die Erst- und

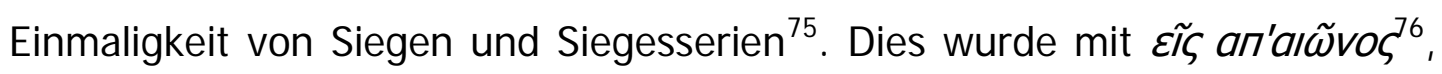

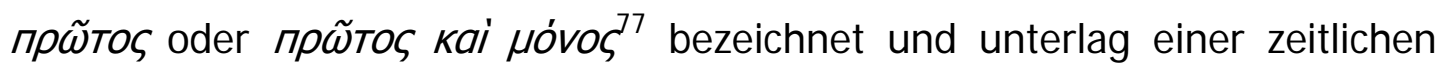
oder personellen Beschränkung ${ }^{78}$. Verständlich erscheint so ein Verweis bei sehr erfolgreichen Ringern, wie etwa bei einem Olympioniken aus Mylana:

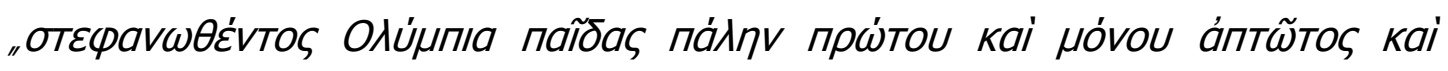

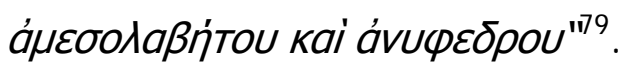

\footnotetext{
72 Vgl. R. KNAB, Periodoniken, Nr.43, 52; 55; L. MORETTI, in: Athenaeum 32, 1954, 116 Nr. 8; Vgl. auch H. A. HARRIS, GAA, 123.

73 Vgl. F. MEZÖ, Geschichte, 85 ff.; A. MODRZE, in: RE 15,2 (1932), s. v. Milon 2, 1672 ff.; L. MORETTI, Olympionikai, Nr. 122; W. DECKER, Sport, 65.

74 Vgl. J. EBERT, Epigramme, 182; D. C. YOUNG, Myth, 136. Falsch H. A. HARRIS, GAA, 111, und J. SWADDLI NG, Games, 59, die Milon nur als fünffachen Periodoniken sehen.

75 J. EBERT, Epigramme, 139, setzt die statistische Erfassung der Sieger als sachliche Vorbedingung voraus.

76 L. ROBERT, Études épigraphiques et philologiques, Paris 1938, 111: „Cette innocente formule signifie: le premier de tous les temps passés, l'unique, l'inégalé; le supérieur à tous ses prédécesseurs. Tout homme qui vient de battre un record est, en ce domaine et jusqu'à

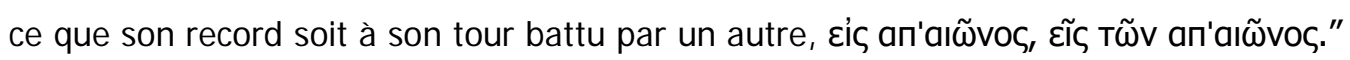

77 M. N. TOD, Greek Record-Keeping and Record-Breaking, in: CQ 53, 1949, 112: „... Hóvo

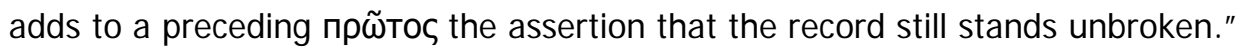

78 Pind. Nem. 6, 17 f.; IG 12,1, 841; IGR 4, 1344; SEG 13, 516; 19,6811; vgl.L. MORETTI, IAG, Nr. 47 f.; J. EBERT, Epigramme, Nr. 72 f.; L. ROBERT, in: RPh 4, 1930, 42; DERS., in: REG 79, 1966, 112 ff.; DERS., Monnais grecques, Paris 1967, 114 f.; H. BUHMANN, Sieg, 75. 79 L. MORETTI, Olympionikai, Nr. 957;vgl.L. ROBERT, Étud. anat., 537; DERS., Lucillius, $250 \mathrm{f}$.
} 


\subsubsection{Boxen}

Der Faustkampf ${ }^{1}$ kannte im Gegensatz zum Ringen und dem Pankration ${ }^{2}$ die wettkampfmäßige Verwendung eines Sportgerätes ${ }^{3}$, nämlich der Faustwehr. Die Jahrhunderte währende historische Entwicklung der Boxhandschuhe ${ }^{4}$ führte zu einer allgemeinen Verschärfung des Kampfes $^{5}$ und brachte so zwangsläufig eine Veränderung der Kampftaktik mit sich ${ }^{6}$. Da die

${ }^{1}$ Zu Boxkampfdarstellungen in der Situlenkunst vgl. J. JÜTHNER, AL 1, 58 ff.; R. PATRUCCO,
Sport, 245 ff. Zum Boxkampf bei den Etruskern vgl. J.-P. THUI LLIER, in: MEFRA 86, 1974,
41 ff.; DERS., in: Stadion 7.2, 1981, 177 f.; DERS., Jeux athlétiques, 181 ff. Zu Suet. Aug.
45,5, wo von pugiles, latinischen Boxern und von Catervakämpfen die Rede ist, vgl.
E. MÄHL, Gymnastik und Athletik im Denken der Römer, Amsterdam 1974, 33; G. PFISTER,
Die Erneuerung der römischen iuventus durch Augustus, Diss. Regensburg 1977, 14 f.; Vgl. auch I. WEILER, Sport, 251 (pugiles Latini). CIL 10, 1074 führt pugiles catervarios und pyctas im Rahmen eines munus an.

${ }^{2}$ Vgl. aber H. A. HARRIS, GAA, Fig. 21, wo scheinbar Pankratiasten mit einer Faustwehr ausgerüstet sind.

3 Zur Bekleidung mit einem Schurz und der Verwendung eines Kopfschutzes vgl. E. N. GARDINER, $A A W$, Fig. 182; J. JÜTHNER, $A L$ 2, Abb. 2 u. 3; R. PATRUCCO, Sport, 230 u. 264; F. BREIN, GL 2, 114; I. WEILER, Sport, 251. Vgl. allgemein zu weniger bekannten Sportgeräten W. RUDOLPH, Antike Sportgeräte, in: Klio 48, 1967, 81 ff.

${ }^{4}$ Hom. II. 23; Paus. 8, 40, 3; Philostr. gymn. 10; vgl. J. JÜTHNER, Antike Turngeräthe, Wien 1896, 65 ff.; E. N. GARDINER, GASF, 402 ff.; S. MENDNER, Boxhandschuhe im Altertum, in: Gymnasium 60, 1953, 20 ff.; E. K. BORTHWICK, A Note on Boxing Gloves, in: CR 14, 1964, 142; W. RUDOLPH, Kampfsport, 8 ff.; L. ROBERT, Lucillius, 211 f.; R. PATRUCCO, Sport, 245 f.; M. POLIAKOFF, Terminology, 11 ff.; T. F. SCANION, in: Stadion 8-9, 1982-3, 31 ff. Nach Plat. leg. 8, 830e und Poll. 3, 148; 150 fügten Faustkämpfer zur Verstärkung der Wirkung ihrer Schläge in die Fausthandschuhe Bleikugeln ein.

5 Der Faustkampf mit dem caestus wird dem Gladiatorenwesen zugewiesen; vgl. E. N. GARDINER, GASF, 410; DERS., AAW, 210 f.; J. JÜTHNER, in: Die Antike 15, 1939, 255; DERS./E. MEHL, in: RE Suppl. 9 (1962), s. v. Pygme, 1323 f.; W. RUDOLPH, Kampfsport, 10, n. 21.

${ }^{6}$ Vgl. E. N. GARDINER, GASF, 414; DERS., AAW, 199; W. RUDOLPH, Kampfsport, 8. Einen ausführlichen Vergleich der Darstellungen des Faustkampfes bei Homer, bietet J. BÖHM, Die Leibesübungen im Dionysosepos des Nonnos von Panopolis, Diss. Wien 1947, 161 ff. 
Kontrahenten gleich ausgerüstet waren ${ }^{7}$, blieb zumindest die Chancengleichheit gewahrt. Im Organisatorischen gab es viele Gemeinsamkeiten mit Ringkampf und Pankration: Man kannte nur eine Einteilung in Alterklassen und unterschied nicht in Gewichtsklassen. Vor jeder Wettkampfrunde wurden die Paarungen stets neu ausgelost ${ }^{9}$ Das Kampfgeschehen fand in einem begrenzten Raum statt ${ }^{10}$ und wurde gemäß dem Regelwerk ${ }^{11}$ von den Kampfrichtern überwacht ${ }^{12}$. Der Faustkampf kannte weder Runden noch Pausen ${ }^{13}$ und auch keine Punktsiege. ${ }^{14}$ Der Sieg wurde durch das Anzeigen der Aufgabe, ${ }^{15}$ oder den K. o. ${ }^{16}$ ermittelt. Im

${ }^{7}$ Vgl. aber H. SCHÖBEL, Olympia und seine Spiele, Gütersloh $1984^{6}$, Abb. 65, wo einer der beiden Boxer mit bloßen Händen kämpft.

${ }^{8}$ Vgl. E. N. GARDINER, GASF, 416; W. RUDOLPH, Kampfsport, 28; R. PATRUCCO, Sport, 247; I. WEILER, Sport, 251, hält es für möglich, dass man in Rom schon Gewichtsklassen kannte.

9 Paus. 6, 23, 2. Vgl. F. MEZÖ, Geschichte, 191 ff.; N. B. CROWTHER, Rounds and Byes in Greek Athletics, in: Stadion 18.1, 1992, 68 ff. (=Athletica 215 ff.).

${ }^{10}$ Vgl. E. N. GARDINER, AAW, 132; R. PATRUCCO, Sport, Fig. 114; H. W. PLEKET, in: MNIR 36, 1974, 66; W. DECKER, Sport, 73 f., Abb. 32 (Begrenzung des Kampfraumes).

11 Jul. Afric. Ol. 23; Philostr. gymn. 12; vgl. L. MORETTI, IAG, 86 f.; J. EBERT, Epigramme, 168; R. PATRUCCO, Sport, 229. Zu einer Disqualifikation vgl. M. I. FINLEY/H. W. PLEKET, Spiele, 133.

12 Vgl. E. N. GARDINER, AAW, Fig. 180 u. 183; H. A. HARRIS, GAA, Fig. 15 u. 16; R. PATRUCCO, Sport, Fig. 116 u. 118.

${ }^{13}$ Vgl. aber Apoll. Rhod. 2, 85 ff.

14 Vgl. E. N. GARDINER, GASF, 415; H. A. HARRIS, SGR, 22; W. RUDOLPH, Kampfsport, $14 \mathrm{f}$.

${ }^{15}$ Philostr. imag. 2, 6; Paus. 6, 10, 2. Vgl. K. SITTL, Die Gebärden der Griechen und Römer, Leipzig 1890, 219. Zur bildlichen Darstellung der Aufgabe vgl. B. SCHRÖDER, Der Sport im Altertum, Berlin 1927, Taf. 90; E. N. GARDINER, AAW, Fig. 186; C. BLÜMEL, Sport und Spiel bei Griechen und Römern, Berlin 1934, Taf. 11; DERS., Sport der Hellenen, Berlin 1936, Nr. 76 u. 99; H. A. HARRIS, GAA, Fig. 19 a; R. PATRUCCO, Sport, Fig. 100 u. 108; V. OLIVOVÁ, Sport und Spiele im Altertum, München 1984, 100 u. 116. Nach Plut. Lykurg. 19 und Philostr. gymn. 9 war das Eingestehen der Niederlage der Grund für die Nichtteilnahme der Spartaner am Faustkampf und dem Pankration. 
ärgsten Fall fand einer der Kontrahenten den Tod, ${ }^{17}$ was, wenn es nicht als Folge eines Regelverstoßes geschehen war, für den Urheber keine strafrechtlichen Konsequenzen mit sich brachte. ${ }^{18}$

Für den hohen Stellenwert eines Sieges im Faustkampf spricht schon die Tatsache, dass man allein die Zulassung zum Wettkampf, ein Vordringen ins Finale und ein Unentschieden in demselben als Auszeichnung empfand ${ }^{19}$. Bei den siegreichen Athleten finden wir neben dem Lob der Stärke ${ }^{20}$ bzw. der Härte der Schläge ${ }^{21}$ eine Würdigung guter Technik $^{22}$ und einer guten Abwehrarbeit, was v. a. den Ruhm Melankomas begründete, da er durch seine überragenden Defensivkünste die Gegner zur Verzweiflung brachte, indem er extrem lange in der Verteidungsstellung ausharren konnte ${ }^{23}$. Eine

${ }^{16}$ Einen technischen K.o. kannte man nicht; vgl. W. DECKER, Sport, 73.

17 Paus. 6, 9, 6 ff.; vgl. F. MEZÖ, Geschichte, 94 f. u. 189; C. A. FORBES, Accidents and Fatalities in Greek Athletics, in: Classical Studies in Honour of W. A. Oldfather, Urbana 1943, 50; L. MORETTI, Olympionikai, Nr. 174; H. W. PLEKET, in: MNIR 36, 1974, 72; H. BUHMANN, Sieg, 55; R. MERKELBACH, in: ZPE 18, 2, 1975, 110 n. 27; M. BROPHY /R. BROPHY, in: AJPh 106, 1985, $177 \mathrm{ff}$.

18 Vgl. J. JÜTHNER/E. MEHL, in: RE Suppl. 9 (1962), s. v. Pygme, 1351; J. EBERT, Epigramme, 143; vgl. auch Dig. 9, 2, 3.

19 IGR 3, 1012; CIG 4240; TAM 2,586; SEG 3, 335; vgl. L. MORETTI, IAG, Nr. 83; L. ROBERT, in: RPh 4, 1930, 28 f.; DERS., Inscriptions grecques d'Asie mineure, in: Anatolian Studies presented to W. H. Buckler, Manchester 1939, 334 ff.; DERS., in: Hellenica 11-12, 1960, 334 ff.; DERS., Lucillius, $186 \mathrm{ff.}$

20 Anth. Pal. 6, 256; SEG 11, 1239; 24, 387; vgl.J. EBERT, Epigramme, Nr. 36, 63, 67; W. PEEK, in: MDAl (A) 67, 1942, 240 ff.; L. ROBERT, Lucillius, 268 u. 288; M. I. FINLEY/H. W. PLEKET, Spiele, 82.

21 Paus. 6, 10, $1 \mathrm{f}$.

22 Pind. Ol. 7, 15 f.; Diog. Laert. 8, 47; vgl. J. G. C. ANDERSON, in: JHS 18, 1898, 90, Nr. 26; J. EBERT, Epigramme, Nr. 55; vgl. Nonn. 37, 526 f. zu flinker Beinarbeit.

23 Dion. Chrys. 27, 121; vgl J. H. KRAUSE, Die Gymnastik und Agonistik der Hellenen, Wiesbaden 1832 (ND 1971), 510; N. YALOURIS, Games, 270 f.; M. I. FINLEY/H. W. PLEKET, Spiele, 84 f.; J. JÜTHNER/E. MEHL, in: RE Suppl. 9 (1962) s. v. Pygme, 1340, sprechen in diesem Zusammenhang von einer messbaren Leistung. 
besondere Auszeichnung stellte es dar, wenn man als Boxer keine Schläge

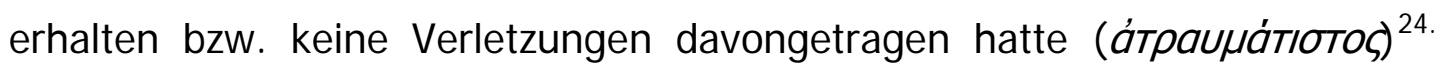
Der Olympionike im Faustkampf der Knaben, Hippomachos, hatte seine drei Gegner besiegt, ohne einen Schlag erhalten zu haben ${ }^{25}$, der Boxer Kleoxenos

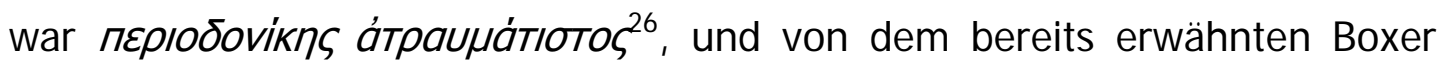
Melankomas wird berichtet, dass er seine gesamte Wettkampflaufbahn unverletzt geblieben sei und wie ein Läufer ausgesehen habe ${ }^{27}$. Seine Überlegenheit spiegelte sich auch darin wider, dass er nicht nur keine Schläge einstecken musste, sondern dass er, ohne selbst Schläge auszuteilen, seine Gegner zur Aufgabe brachte ${ }^{28}$.

Bei den vielen Gemeinsamkeiten der drei schwerathletischen Kampfsportdisziplinen ist es verständlich, dass wir viele aus dem Ringkampf bekannte lobende Worte (Ëníreta ópvavtía/Epitheta ornantia) auch im Boxsport und Pankration antreffen. Die Überlegenheit eines Faustkampfsieges konnte durch den Sieg ohne Kampf (ákoviti) unterstrichen

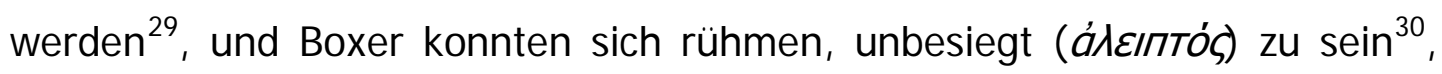

24 Vgl. R. KNAB, Periodoniken, 12; L. ROBERT, Lucillius, 234 f.; H. W. PLEKET, in: Stadion 1.1, 1975, 76; M. I. FINLEY/H. W. PLEKET, Spiele, 83; W. DECKER, Sport, 74 spricht in diesem Zusammenhang von Prädikat und Ehrentitel.

25 Paus. 6, 12, 6.

26 J ul. Afric. Ol. 135; vgl. R. KNAB, Periodoniken, 12; L. MORETTI, Olympionikai, Nr. 569.

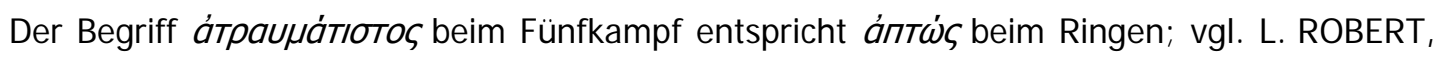
Lucillius, 251.

27 Dion. Chrys. 28, 7; vgl. L. ROBERT, in: Hellenica 11-12, 1960, 338 f. u. 442; DERS., Lucillius, 235; J. JÜTHNER, $A L$ 2, 22; Vgl. im Gegensatz dazu Anth. Pal. 11, 75 ff. einen bis zur Unkenntlichkeit entstellten Boxer.

28 Dion. Chry. 28,7.

29 IvO. 153; Paus. 5, 21, 14; 6, 7, 4; vgl. R. KNAB, Periodoniken, 11; J. JÜTHNER/E. MEHL, in: RE Suppl. 9 (1962), s. v. Pygme, 1348. Quint. Smyrn. 4, 329 bezeichnet einen solchen

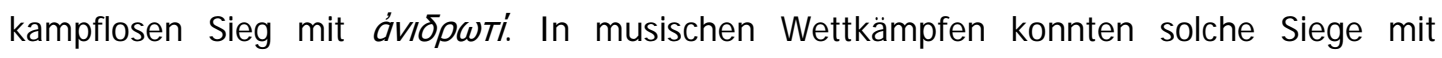

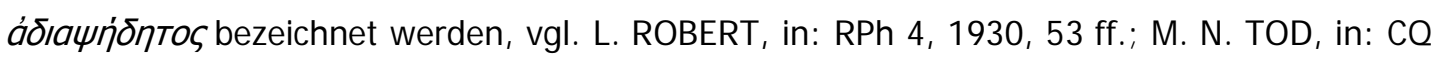
53, 1949, 110; L. MORETTI, IAG, $238 \mathrm{f}$. 


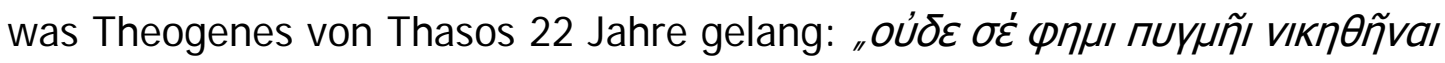

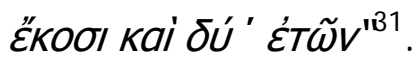

Besonders brillanten Siegen fügte man ein $\varepsilon^{\prime} v \delta o ́ \xi \omega \varsigma$ bei ${ }^{32}$. Bei ungerader Teilnehmerzahl kam es auch beim Boxen zu Finalsiegen mit und ohne

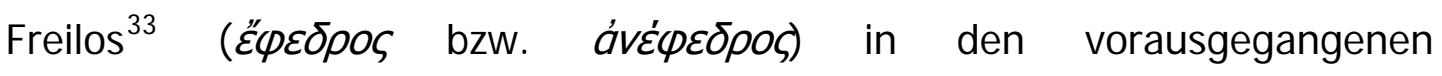
Ausscheidungsrunden ${ }^{34}$, und auch die Zahl der Runden, die man gekämpft hatte, bzw. die Zahl der überwundenen Gegner finden sich des Öfteren bei erfolgreichen Boxern vermerkt ${ }^{35}$. Ein Ausdruck anerkannter Überlegenheit war es, wenn man nach einer oder mehreren Ausscheidungsrunden, zumindest vor Erreichen des Finales, die Gegner zu einem freiwilligen Verzicht auf die Fortführung der Kämpfe (und somit auf den Sieg) bewegte:

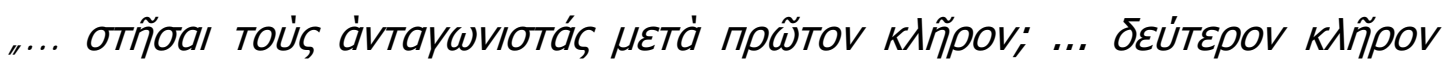
$\kappa T \lambda .{ }^{136}$

30 IG 14,1105; Dion. Chrys. 28, 9; vgl. R. KNAB, Periodoniken, 13; L. ROBERT, in: Hellenica 11-12, 1960, $338 \mathrm{f}$.

31 Syll.3 36; IG 12, 8; vgl. W. PEEK, MDAl (A) 67, 1942, 240 ff.; L. MORETTI, IAG, Nr. 21; J. EBERT, Epigramme, Nr. 37. Vgl. auch D. C. YOUNG, Myth, $96 \mathrm{f.}$

32 Vgl. zahlreiche Beispiele bei L. ROBERT, in: Hellenica 11-12, 1960, 351 ff.; vgl auch J. JÜTHNER, WS 24, 1902, 290.

33 Zu der Häufigkeit, mit der man in den Genuss eines Freiloses innerhalb einer Konkurrenz kommen konnte; J. EBERT, Epigramme, 228 f.; N. B. CROWTHER, in: Stadion 18.1, 1992, $72 \mathrm{ff}$. (=Athletika $218 \mathrm{ff}$.).

34 Offen bleibt, ob ein dritter Mann bei Boxkampfdarstellungen auf Vasen ein Ephedros ist oder ob damit lediglich Turnieratmosphäre angezeigt werden soll; vgl. E. N. GARDINER, in:

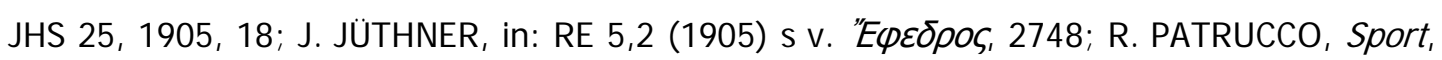
260.

35 IvO 174; vgl. L. MORETTI, IAG, Nr. 33; J. EBERT, Epigramme, Nr. 5; vgl. L. ROBERT, in: Hellenica 7, 1949, 108; N. YALOURIS, Games, 222.

${ }^{36}$ Vgl. L. ROBERT, in: Hellenica 7, 1949, 105 ff.; J. EBERT, Epigramme, 54 n. 1. 
Faustkämpfer waren häufig auch im Ringen und Pankration erfolgreich ${ }^{37}$ und z. T. auch in anderen Disziplinen, wobei die Laufkonkurrenzen überwogen ${ }^{38}$. Mehrfachsieger an einem Tag in verschiedenen Disziplinen und/oder verschiedenen Altersklassen wurden detailliert hervorgehoben ${ }^{39}$ oder durch

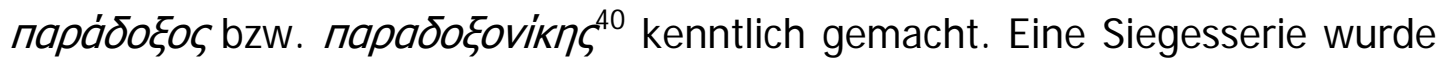
meist mit катá тó $\dot{\xi} \xi \tilde{\zeta}$ zum Ausdruck gebracht ${ }^{41}$.

Die zahlenmäßige Erfassung der Siege war vielfältig und reichte vom Verweis auf einen oder einige Siege über die Angabe der Siege bei den großen panhellenischen Spielen bis zu ausführlichen Siegeslisten - gelegentlich in augenfällig tabellarischer Forrn ${ }^{42}$-, die kaum einen Sieg ausließen und mit den verschiedensten Ruhmesbekundungen versehen waren ${ }^{43}$. Oft wurden

37 L. MORETTI, IAG, Nr. 21; 29; 40; 47; 55; 62; 68; 84; J. EBERT, Epigramme, Nr. 37; 47; 67; 70.

${ }^{38} \mathrm{CIG}$ 4472; IGR 3, 1012; Paus. 6, 11, 5; vgl. L. MORETTI, IAG, Nr. 21; 85.

${ }^{39}$ Anth. Pal. 9, 588; IG 12, 3, 390; vgl. Th. REINACH, in: RA 1916, 354 ff.; L. MORETTI, IAG, Nr. 40; 55; 69; L. ROBERT, in: Hellenica 11-12, 1960, 347 f.; J. EBERT, in: WZ Halle 15,

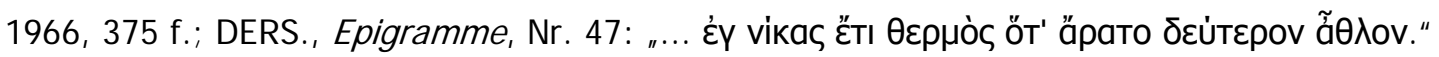
Natürlich war es auch im Boxkampf eine außergewöhnliche Leistung, wenn man als Knabe den Männern zugeteilt wurde und siegte, vgl. Jul. Afric. Ol. 48; L. ROBERT, in: Hellenica 7, 1949, 113. Vgl. auch Paus. 6, 3, 1 zu einem außergewöhnlich jungen Olympioniken im Faustkampf der Knaben.

40 IG 14, 110, 5; IGR 4, 1519; vgl. L. MORETTI, IAG, Nr. 69; 83 f.; vgl. Th. REINACH, in: RA 1916, 354 ff.; R. KNAB, Periodoniken, 13; L. ROBERT, in: RPh 4, 1930; 44 ff. Hier sei auch an die Siege dreier Brüder am selben Tag in Olympia erinnert, Gell. 3, 15, 3; vgl. C. A. FORBES, in: Class. Stud. Oldfather, $50 \mathrm{f}$; W. RUDOLPH, $\mathrm{Zu}$ den Formen des Berufssports zur Zeit der Poliskrise, in: E. Ch. WELSKOPF (Hrsg.), Poleis, 1481.

${ }^{41}$ IG 3, 128; vgl. L. ROBERT, in: RPh 4, 1930, 37.

${ }^{42}$ Vgl. J. EBERT, Epigramme, Nr. 37 den Prosa-Katalog.

43 Paus. 6, 10, 1 ff.; 6, 13, 8; in: SEG 16, 289; 17, 201; 23, 262; L. MORETTI, IAG, Nr. 13 f.; J. JÜTHNER, Epigramme, 16 f.; L. SEMMLINGER, Weih-, Sieger- und Ehreninschriften aus Olympia und seiner Umgebung, Diss. Erlangen-Nürnberg 1974, Nr. 64; H. BUHMANN, Sieg, 123.6. 
diese Siegeskataloge ergänzt durch den Zusatz пגعıбтоviкn $\varsigma^{44}$ oder Formeln

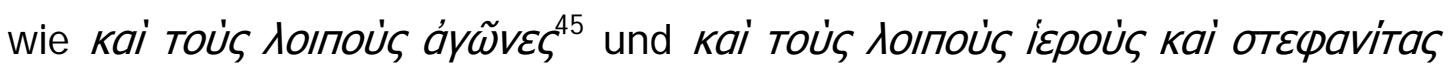

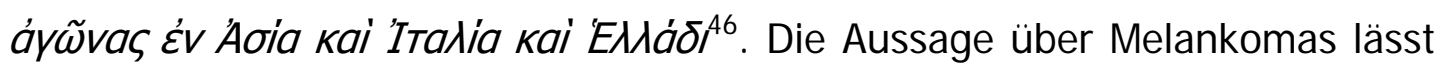

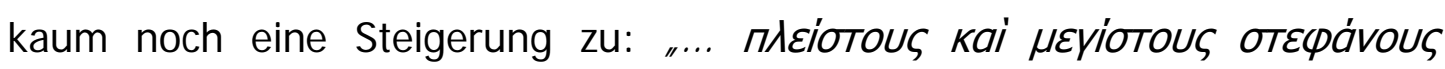

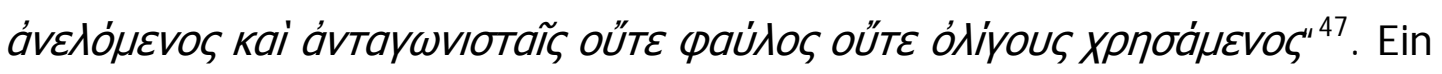
Siegeskatalog konnte aber auch mit einer klaren Zahlenangabe abschließen:

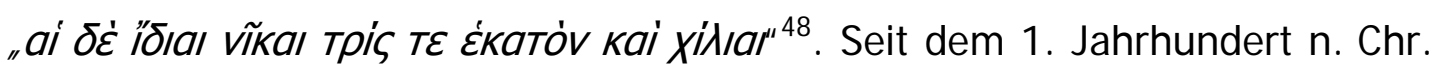
findet sich häufig die Angabe der Zahl der Siege entsprechend dem

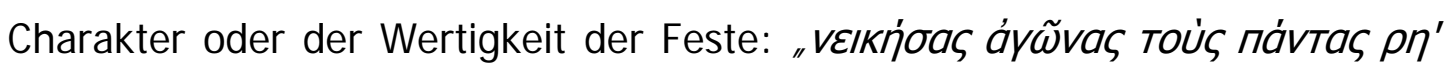

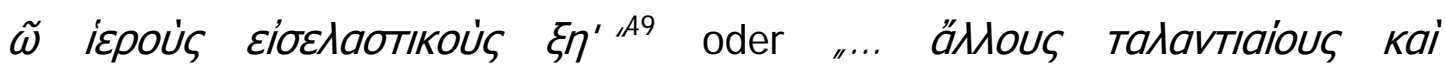

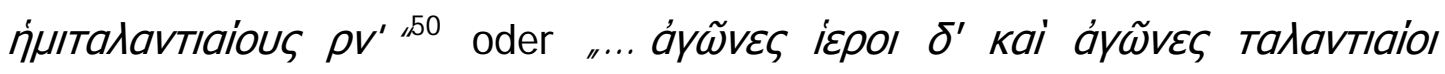
$\mu a^{\prime \prime 51}$. Eine besonders hohe Summe an Siegen hatten oft die Periodoniken aufzuweisen, von denen uns ein knappes Dutzend aus dem Bereich des Boxsports bekannt ist ${ }^{52}$. Wie schwierig es war, dies zu erreichen, wird am

44 CIG 4240; TAM 2, 586; vgl. L. MORETTI, IAG, Nr. 83 f.; L. ROBERT, in: RPh 4, 1930, 44 ff.; DERS., in: Hellenica 5, 1948, 63.

${ }^{45}$ GIBM 929; vgl. L. MORETTI, IAG, Nr. 49 u. 62; vgl. Th. KLEE, Geschichte, 76.

46 Vgl. R. KNAB, Periodoniken, 37; L. MORETTI, Olympionikai, Nr. 725. Zum Titel

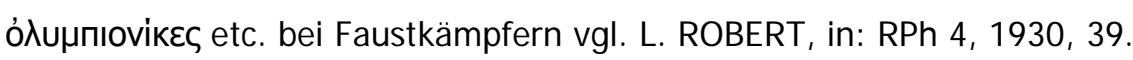

47 Dion. Chrys. 28, 9. Nach Pind. Nem. 6, 24 ff. hatte das Haus der Bassiden die meisten Faustkampfsiege zu verzeichnen; vgl. K. KRAMER, Studien, 89; W. DECKER, Sport, 75.

48 W. PEEK, in: MDAl (A) 67, 1942, 240 ff.; L. MORETTI, IAG, Nr. 21; J. EBERT, Epigramme, 37. Über die Glaubwürdigkeit dieser Angabe vgl. L. MORETTI, IAG, 53. Paus. 6, 11, 5 spricht von 1400 Siegen; Plut. praec. rei publ. ger. 15, 7 gibt 1200 Siege an. 49 IGR 4, 1519; vgl. L. ROBERT, in: RPh 4, 1930, 44 ff.; L. MORETTI, IAG, Nr. 84. In Teil C der Inschrift werden die Siege bei den Heiligen Wettkämpfen unter Knaben und Männer aufgesplittet; zu den dabei sich nicht schlüssig ergänzenden Zahlenangaben vgl. L. MORETTI, IAG, 247.

${ }^{50}$ Vgl. Th. REINACH, in: RA 1916, 354 ff.; L. MORETTI, IAG, Nr. 69. H. BUHMANN, Sieg, 32.

51 IGR 3, 1012; vgl. L. ROBERT, in: Hellenica 2, 1946, 70 f.

${ }^{52}$ Vgl. R. KNAB, Periodoniken, Nr. 2; 4; 8; 9; 12; 16; 22; 26; 27; 28; 30; 33; 43; 58; $59 ; 63$. Vgl. auch Paus. 6, 15, 1 zu einem erst zwanzigjährigen Periodoniken im Faustkampf. 
Beispiel des oben erwähnten Ausnahmeathleten Theogenes ${ }^{53}$ deutlich. Dieser überaus erfolgreiche Faustkämpfer, der insgesamt 1.300 Siege vorzuweisen hatte, war lediglich zweifacher Periodonike ${ }^{54}$. Im Bereich des Boxsports finden wir mit Moschos von Kolophon eine einzigartige Erscheinung im

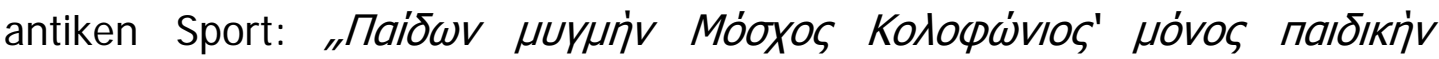

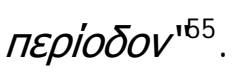

Der Hinweis auf die Erst- und/oder Einmaligkeit der Leistung oder bestimmter Umstände - häufig mit án' aí̃vos verbunden - wurde in äußerst vielfältiger Weise dargeboten. Der Kreis der angesprochenen Personen, denen man durch seine Leistung voranstand, konnte sich auf die Heimatgemeinde beschränken ${ }^{56}$, zielte aber häufiger auf die Athletengemeinschaft ${ }^{57}$, alle Hellenen ${ }^{58}$, alle Knaben, Bartlosen und Männer ${ }^{59}$ oder die gesamte Menschheit ${ }^{60}$ ab. Dreifachen Ruhm in letzterer Weise wurde Theogenes von Thasos in dem inm gewidmeten Siegerepigramm

53 Vgl. M. LAUNEY, in: RA 1941, 22 ff.; N. YALOURIS, Games, 264 f.

${ }^{54}$ Vgl. L. MORETTI, IAG, 53; W. DECKER, Sport, 110.

55 Jul. Afric. Ol. 145; vgl. R. KNAB, Periodoniken, 8 u. 35; L. MORETTI, Olympionikai, Nr. 602.

${ }^{56}$ CIG 4472; IGR 3, 1012; vgl. L. MORETTI, IAG, Nr. 85; L. ROBERT, Lucillius, 275 n. 2. Zu

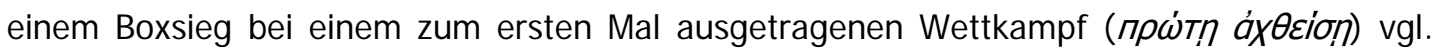
L. MORETTI, IAG, Nr. 85; vgl. auch L. ROBERT, in: RPh 4, 1930, 28 u. 46 n. 1; DERS., in: Hellenica 11-12, 1960, 360.

${ }^{57}$ Vgl. L. MORETTI, IAG, Nr. 62; ebd. 163 versteht L. MORETTI darunter nicht die Gesamtheit der Sterblichen, sondern die Athletengemeinschaft.

58 Anth. Pal. 9, 588; vgl. L. ROBERT, in: RPh 77, 1967, 25 f.; DERS., Lucillius, 195 f.

${ }^{59}$ Vgl. Th. REINACH, in: RA 1916, 254 ff.; L. MORETTI, IAG, Nr. 69.

60 IGR 4, 1519; L. ROBERT, in: RPh 4, 1930, 44 ff.; L. MORETTI, IAG, Nr. 84; W. DECKER, Sport, 111. 
zugestanden: Er hatte sowohl in Olympia als auch bei den Isthmien und

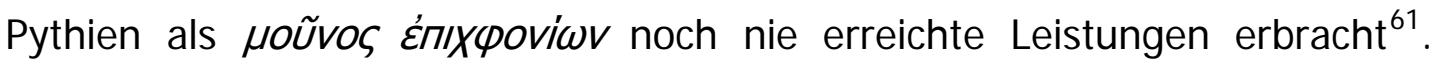

${ }^{61}$ Vgl. W. PEEK, in: MDAI (A) 67, 1942, 240 ff.; L. MORETTI, IAG, Nr. 21; J. EBERT, Epigramme, Nr. 37. J. JÜTHNER/E. MEHL, in: RE Suppl. 9 (1962), s. v. Pygme, 1348, sprechen hier von einem Weltrekord in der heutigen Sprache." 


\subsubsection{Pankration}

Das Pankration erfreute sich im vielfältigen Kanon der Sportarten besonderer Beliebtheit ${ }^{1}$. Diese schwerathletische Disziplin aus Elementen des Ring- und Boxkampfes $^{2}$ mit dem Zusatz von Beintechniken ${ }^{3}$ kannte nur eine Einteilung in Altersklassen ${ }^{4}$. Die Zuordnung der Kampfpaare erfolgte durch das Los, ${ }^{5}$ und die Kämpfe fanden ohne Sportgeräte ${ }^{6}$ im $\sigma \kappa a ́ \mu \mu a^{7}$ statt. Mit Ruten ausgestattete Kampfrichter ${ }^{8}$ sorgten für die Einhaltung der Regeln, ${ }^{9}$ und wie beim Boxkampf wurde ohne Pause bis zur Aufgabe oder Kampfunfähigkeit einer der Gegner gekämpft ${ }^{10}$.

\footnotetext{
${ }^{1}$ Philostr. imag. 2, 6; Pind. Nem. 11, 21; Thuk. 5, 49; Cass. Dio 59, 13; Pindar verfasste acht Siegeroden für Pankratiasten, in denen er stets das athletische Ideal der Kämpfer gerühmt hat. Zu hohen Siegprämien im Pankration vgl. L. ZEHEN, in: RE 18,3 (1949), s. v. Panathenaia, 476; H. A. HARRIS, GAA, 109; J. SWADDLING, Games, 60. Vgl. auch M. I. FINLEY/H. W. PLEKET, Spiele, $89 \mathrm{f}$.

2 Plut. quaest. conv. 2, 4, 638 d; Philostr. gymn. 11. Vgl. W. RUDOLPH, Kampfsport, 66. Zur

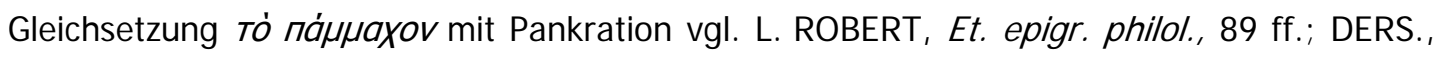
in: Hellenica 2, 1946, 140 f.; DERS., Lucillius, 261.

3 Theokr. 22, 66; Suda s. v. паратıаoтаї

${ }^{4}$ Vgl. W. RUDOLPH, Kampfsport, 3; J. EBERT, in: Stadion 5, 1, 1981, 16.

${ }^{5}$ Lukian. Hermot. 40; vgl. J. JÜTHNER, in: RE 18, (1949), s. v. Pankration, 621.

6 Gelegentlich finden sich Darstellungen von Pankratiasten, die mit einer Faustwehr ausgerüstet sind; vgl. E. N. GARDINER, AAW, Fig. 200; H. A. HARRIS, GAA, Fig. 21; M. POLIAKOFF, Terminology, 11 u. 19.
}

${ }^{7}$ Vgl. L. MORETTI, IAG, 233; F. BREIN, GL 2,117.

${ }^{8}$ Vgl. E. N. GARDINER, in: JHS 26, 1906, Fig. 1; DERS., GASF, Fig. 151 f.; DERS., AAW, Fig. 188 f.; H. A. HARRIS, GAA, Taf. 13 a; 14 a; 15 f; 18; 19c; 20; R. PATRUCCO, Sport, Fig. 150 u. 157.

9 Lukian. Demot. 49; Paus. 3, 14, 10; Philostr. imag. 2, 6; vgl. E. N. GARDINER, GASF, 435 f.; J. JÜTHNER, in: RE 18, 3 (1949), s. v. Pankration, 621; H. A. HARRIS, GAA, 106; W. RUDOLPH, Kampfsport, 65 f.; R. PATRUCCO, Sport, 310; R. MERKELBACH, Pankration, in: ZPE 5, 1970, 30 f.; W. DECKER, Sport, 76.

10 Philostr. gymn. 9; vgl. J. JÜTHNER, Philostratus über Gymnastik, Leipzig/Berlin 1909, 203 f.; DERS., in: RE 18.3 (1949), s. v. Pankration, 620; J. SWADDLING, Games, 60; 
Bei dem hohen Stellenwert des Pankration war es manchen Athleten schon

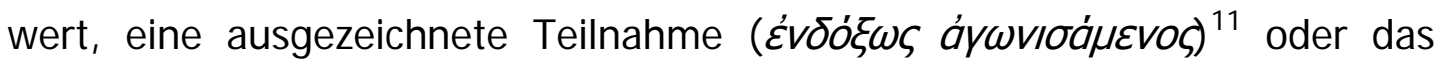

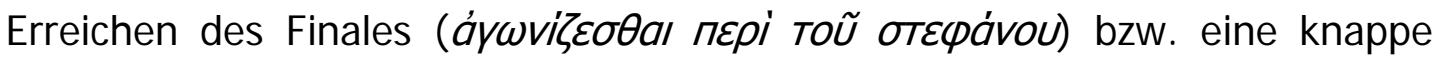

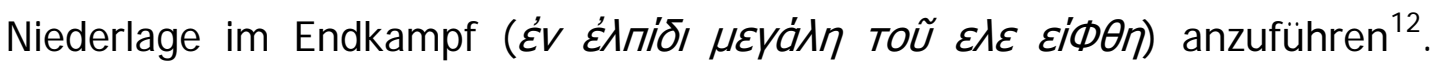
Konnte auch ein Unentschieden Anerkennung finden ${ }^{13}$, so galt doch besondere Wertschätzung den Siegern, die mit ihrer Kraft ${ }^{14}$ oder besonderen Techniken ${ }^{15}$ oder ihrem aufopferungsvollen Mut bis zum Tod $^{16}$ erfolgreich waren. Die aus dem Ringen und Boxen bekannten Zusatzbemerkungen zu

M. POLIAKOFF, Terminology, 10. Vgl. auch Plut. Lykurg. 19 betreffs der Nichtteilnahme der Spartaner an Wettkämpfen im Pankration.

11 Vgl. L. ROBERT, Anat. Stud. Buckler, 237 f.; DERS., in: Hellenica 11-12, 1960, 357; DERS., Lucillius, $186 \mathrm{f}$.

12 Syll. 3 1073; vgl. L. ROBERT, in: RPh 4, 1930, 28 f.; DERS., Anat. Stud. Buckler, 239 ff.; DERS., in: Hellenica 11-12,1960, $334 \mathrm{ff.}$

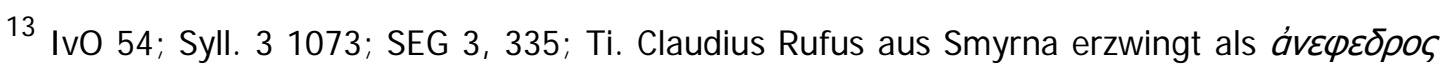
gegen einen zuvor durch das Losglück Begünstigten im Finale ein Unentschieden. Die Eleer preisen inn wie einen Sieger; vgl. L. ROBERT, in: RPh 4, 1930, 27 f.; DERS., Anat. Stud. Buckler, 232; DERS., in: Hellenica 11-12, 1960, 336; DERS., Lucillius, 199; R. MERKELBACH, in: ZPE 15, 1974, 99 ff.; M. I. FI NLEY/H. W. PLEKET, Spiele 190.

14 MAMA 8, 417; SEG 24, 387; L. MORETTI, IAG, Nr. 22; J. EBERT, Epigramme, Nr. 40; 67; vgl. L. ROBERT, in: Hellenica 2, 1946, 139 f., DERS., in: Hellenica 13, 1965, 134 ff. (der Athlet übertrifft alle Vorgänger an Körperkraft und auch psychischer Stärke); R. MERKELBACH, in: ZPE 18.2, 1975, 110.

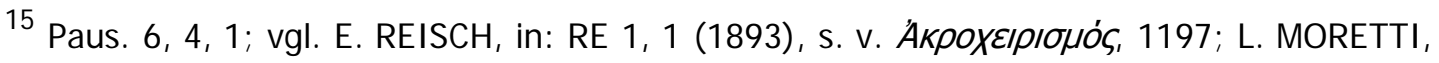
IAG, Nr. 25; R. PATRUCCO, Sport, 322. Zu anderen Spezialtechniken vgl. H. A. HARRIS, GAA, 107; K. KRAMER, Studien zur griechischen Agonistik nach den Epinikien Pindars, Diss. Köln 1970, 98 ff.; N. YALOURIS, Games, 226; J. SWADDLING, Games, 61.

${ }^{16}$ Paus. 8, 40, 1 f.; Philostr. gymn. 2, 6; vgl. F. MEZÖ, Geschichte, 106; C. A. FORBES, in: Class. Stud. Oldfather, 50 ff., L. MORETTI, IAG, 73 f.; M. I. FINLEY/H. W. PLEKET, Spiele, 90; R. H. BROPHY, in: AJPh 99, 1978, 370 ff.; S. G. MILLER, Arete, 27 f.; Vgl. Paus. 8, 40, 1 ff. zu dem legendären Kampf des Arrhachion, der den Sieg mit dem Tod bezahlte. Nach Paus. 6, 5, 4 ff. hatte Pulydamas von Skotussa, ein erfolgreicher Pankratiast, bei einem Schaukampf am Hof des Perserkönigs Dareios II. drei Perser von den sogenannten Unsterblichen, die zusammen gegen inn allein kämpften, getötet. 
den Siegern finden wir ebenso beim Pankration. So wurde ein Sieg durch

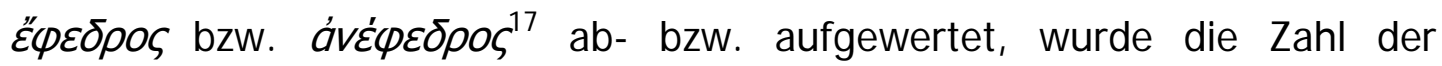
besiegten Kontrahenten angegeben ${ }^{18}$, und Siege, die man in Folge (ката́ тó $\dot{\varepsilon} \xi \tilde{\zeta})$ erzielt hatte, wurden besonders vermerkt ${ }^{19}$. Ruhmvolle Siege wurden als solche mit $\varepsilon^{\prime} v \delta o ́ \xi \omega \varsigma$ herausgehoben ${ }^{20}$, und natürlich schmückten sich auch

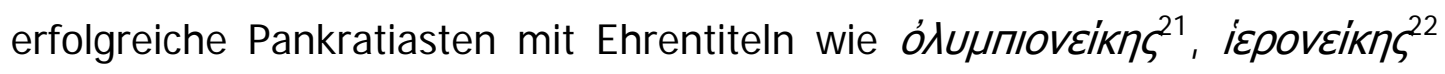
etc. Ebenso galt es im Pankration als besonders gut, wenn der Sieg dem Athleten aufgrund anerkannter Überlegenheit ohne Kampf (ákoVITi) ${ }^{23}$ zufiel. Was dem staublosen Sieg im eigentlichen Sinne entspricht, heißt in der

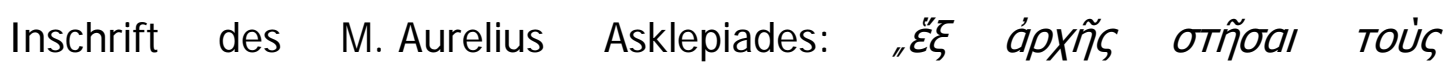

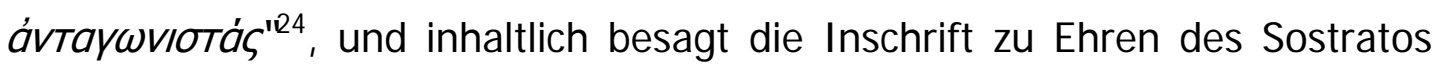

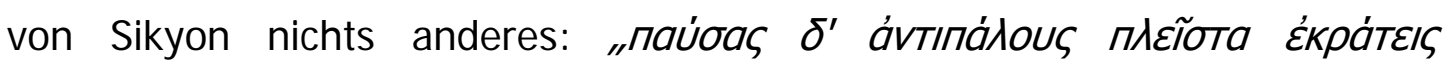
ámaxei' ${ }^{\prime 25}$. Etwas weniger galt ein Sieg, bei dem man die Kontrahenten erst ${ }^{17}$ IvO 54; 227; Syll 3 1070; vgl. B. HAUSSOULLIER, in: BCH 6, 1882, 448 Nr. 76; R. KNAB, Periodoniken, 15; L. MORETTI, Olympionikai, Nr. 822; J. EBERT, Epigramme, 228 f.; N. YALOURIS, Games, 123.

${ }^{18}$ IvO 225;vgl. J. G. C. ANDERSON, in: JHS 3, 1913, 282, Nr. 10; L. MORETTI, IAG, Nr. 64;

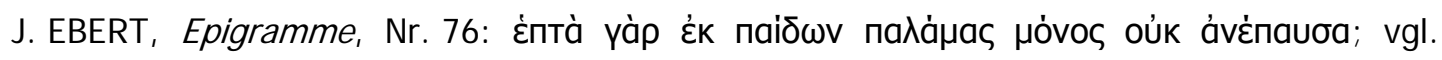
L. ROBERT, Lucillius, $196 \mathrm{f}$.; Zu bildlichen Darstellungen eines Ephedros vgl. E. N. GARDINER, in: JHS 26, 1906, Taf. 3 u. 4; R. PATRUCCO, Sport, Fig. 151 ff.

19 Paus. 8, 40, 1; vgl. L. ROBERT, in: Hellenica 7, 1949, 105; J. EBERT, Epigramme, 235.

${ }^{20}$ CIG 3503; IGR 4, 1968; 1609; MAMA 4, 132; vgl. L. ROBERT, in: Hellenica 11-12, 1960, $352 \mathrm{ff}$.

${ }^{21}$ Vgl. L. ROBERT, in: Hellenica 5, 1948, 62 f.; zu dem Titel ápıoтoveikns in der Inschrift eines erfolgreichen Pankratiasten vgl. L. ROBERT, in: Eos 48.2, 1957, $229 \mathrm{ff}$.

22 MAMA 8, 417; vgl. L. ROBERT, in: Hellenica 13, 1965, 134; K. KRAMER, Studien, 133 n. 6; R. MERKELBACH, in: ZPE 13, 1974, 276.

${ }^{23}$ Paus. 6, 11, 4; Jul. Afric. zu Ol. 118; Plin. n. h. 35, 139; IG 14, 1101; vgl. J. JÜTHNER, in: Glotta 29, 1942, 74 f.; Nach Xenophon Ages. 6, 3 hatte ein ákovití-Sieg die gleiche Geltung wie ein Sieg durch Kampf.

${ }^{24}$ IG 14,1102; IGR 1,153; vgl. L. MORETTI, IAG, 234, Nr. 79; vgl. J. JÜTHNER, in: Glotta 29, 1942, 77.

${ }^{25}$ Vgl. B. HAUSSOULLIER, in: BCH 6, 1882, 446 ff., H. POMTOW, in: Klio 9, 1909183 ff.; L. MORETTI, IAG, Nr. 25; SEG 23, 325; J. EBERT, Epigramme, Nr. 39. 
nach der ersten oder zweiten Runde zum Verzicht bewogen hatte ${ }^{26}$. Besonderes Gewicht wurde der Unbesiegbarkeit beigemessen: „દ́v бтаסioı

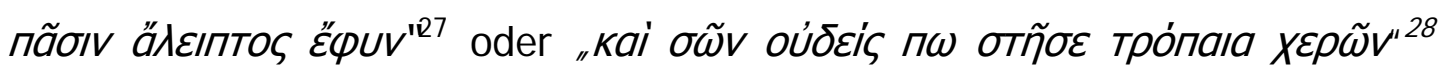

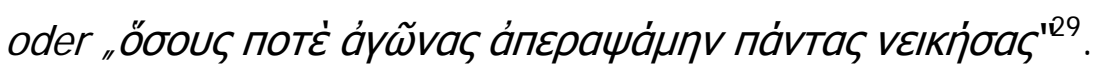

Neben der Betonung der außergewöhnlichen Siege und der Unbesiegbarkeit finden sich gerade in Darstellungen der Karrieren von Pankratiasten Häufungen von Formeln, die die Ausnahmestellung des Athleten dokumentieren sollen ${ }^{30}$. So wird von dem Pankratiasten P. Aelius Aristomachus $^{31}$ u. a. berichtet, dass er niemals unentschieden gekämpft habe, sei es durch Schiedsspruch der Kampfrichter, sei es durch Absprache

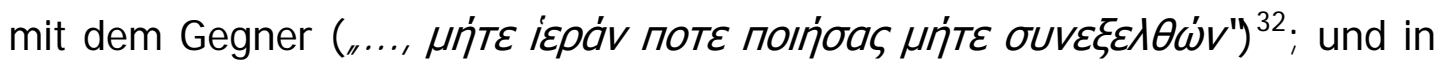
einer der interessantesten agonistischen Inschriften zu Ehren des bereits oben erwähnten Pankratiasten M. Aurelius Asklepiades ${ }^{33}$, der von seinen Gegnern nicht von der Kampffläche gedrängt werden konnte

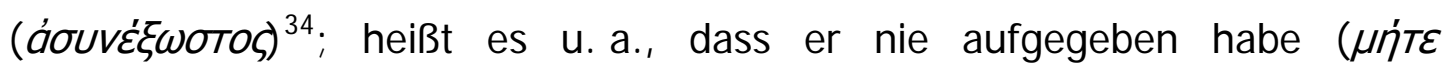

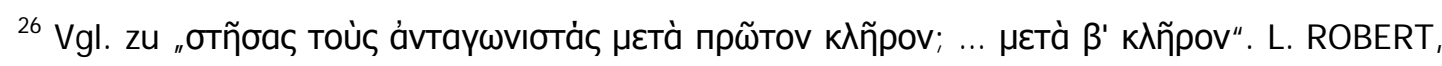
in: Hellenica 7, 1949, 105 ff.; L. MORETTI, IAG, 234; M. I. FINLEY/H. W. PLEKET, Spiele, 116.

${ }^{27}$ Vgl. L. MORETTI, IAG, Nr. 71 b; J. EBERT, Epigramme, Nr. 78; vgl. L ROBERT, in: Hellenica 11-12, 1960, 340; DERS., Étud. anat., 121 ff.; DERS., Lucillius, 185.

${ }^{28}$ Vgl. J. EBERT, Epigramme, Nr. 43; M. BROPHY/R. BROPHY, in: AJ Ph 106, 1985, 173.

29 IG 14, 1102; in: IGR 1, 153; L. MORETTI, IAG, Nr. 79; vgl. L. ROBERT, Lucillius, 183.

30 Vgl. E. N. GARDINER, $A A W, 110$ ff., der aber in einigen Interpretationen der Siegerinschriften zu korrigieren ist.

${ }^{31}$ Vgl. R. KNAB, Periodoniken, 81; L. MORETTI, in: Athenaeum 32, 1954, 115 ff.; DERS., Olympionikai, Nr. 838.

32 IVM 180; vgl. L. ROBERT, in: RPh 4, 1930, 28; DERS., in: Hellenica 6, 1948, 46; L. MORETTI, IAG, Nr. 71; G. E. BEAN, in: AJA 60, 1956, 198; J. EBERT, Epigramme, Nr. 78. 33 IG 14, 1102; IGR 1, 153; L. MORETTI, IAG, Nr. 79; W. DECKER, Sport, $141 \mathrm{f}$.

34 Vgl. R. KNAB, Periodoniken, 13; L. MORETTI, IAG, 232; G. E. BEAN, in: AJ A 60, 1956, 199; M. POLIAKOFF, A $\Sigma \Psi N E=\Omega \Sigma T O \Sigma$, in: ZPE 44, 1981, 78 ff. 


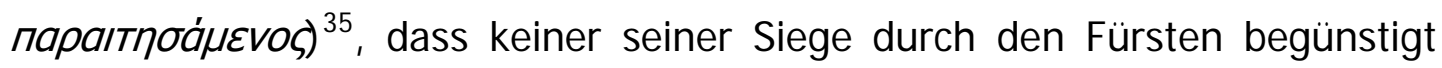

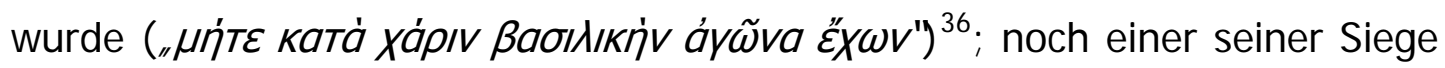

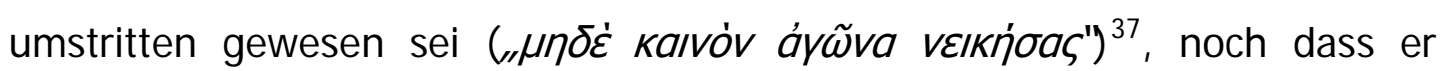
jemals zum Revanchekampf herausgefordert wurde oder ein von ihm herausgeforderter Pankratiast sich dem Kampf gestellt habe („„urite

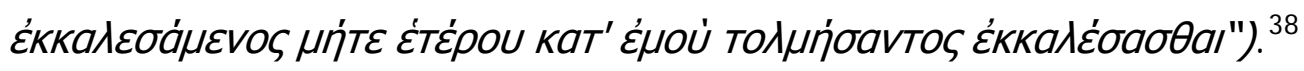

Auch unter den Pankratiasten finden wir zahlreiche Athleten, die neben Siegen in ihrer Spezialdisziplin Erfolge in anderen schwerathletischen ${ }^{39}$ oder weiteren Disziplinen ${ }^{40}$ vorweisen konnten. Mehrfachsiege an einem Tage in verschiedenen Disziplinen ${ }^{41}$ oder Altersklassen ${ }^{42}$ wurden detailliert

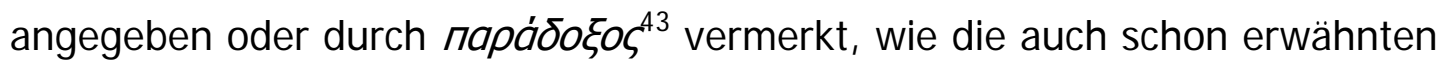
Heraklessieger ${ }^{44}$ dazu gehörten.

35 Vgl. L. ROBERT, Anat. Stud. Buckler, 244 n. 6; DERS., in: Hellenica 7, 1949, 110; J. EBERT, Epigramme, 143.

${ }^{36}$ Vgl. E. N. GARDINER, AAW, 112; L. ROBERT, Lucillius, $191 \mathrm{f}$.

${ }^{37}$ Vgl. H. A. HARRIS, in: JHS 82, 1962, 19.

38 Vgl. E. N. GARDINER, AAW, 112; L. ROBERT, in: Hellenica 7, 1946, 109 n. 2; L. MORETTI, IAG, 232; J. EBERT, in: Stadion 7.2, 1981, 207; vgl auch zu dem Schwerathleten M.Aur.Demostratos Damas J.BOUSQUET,BCH 116,Inscriptions de Delphes,in:BCH 116,1992,177ff.

39 Anth. Pal. 9, 588; IG 4, 428; 14, 747; IGR 1, 446; 4, 1519; vgl. L. ROBERT, in: RPh 4, 1930, 44 ff.; DERS., in: Hellenica 11-12, 1960, 347 f.; Th. KLEE, Geschichte, 54 ff.; L. MORETTI, IAG, Nr. 40, 51; 55, 62; 68; 84; J. EBERT, Epigramme, Nr. 37; 47; 67.

40 Paus. 6, 11, 5; 6, 14, 2 f.; L. MORETTI, IAG, Nr. 21; J. EBERT, Epigramme, Nr. 37.

${ }^{41}$ IG 12, 390; vgl. L. MORETTI, IAG, Nr. 55; L. ROBERT, in: Hellenica 11-12, 1960, 347.

42 Paus. 6, 14, 2 f.; Syll. 3, 36; L. MORETTI, IAG, Nr. 21; vgl. R. KNAB, Periodoniken, 19ff.;

L. ROBERT, Anat. Stud. Buckler, 230 ff.; DERS., in: Hellenica 7, 1949, 112; L. MORETTI, Olympionikai, Nr. 799; R. MERKELBACH, in: ZPE 14, 1974, 91 ff.

43 IG 14, 747; 1102; 1104 f.; IGR 1, 446; vgl. L. MORETTI, IAG. Nr. 68; 72; vgl. O. RIEMANN, in: BCH 1, 1877, 290, n. 75; O. LIERMANN, Analecta epigraphica et agonistica, Diss. Halle 1889, 88 ff.; L. ROBERT, in: BCH 51, 1928, 420 ff.; DERS., in: RA 1934, 53; DERS., in: Hellenica 5, 1948, 62; R. MERKELBACH, in: ZPE 14, 1974, $94 \mathrm{f}$.

${ }^{44}$ Vgl. C. A. FORBES, in: AJ Ph 60, 1939, 473. 
Die Aufzählung der Siege konnte knapp ausfallen ${ }^{45}$, sich auf die Heiligen Kranzspiele ${ }^{46}$ oder auf wenige wichtige Siege ${ }^{47}$ beschränken oder in ausführlichen Siegeskatalogen ${ }^{48}$ den Leser bestens über die jahrelang erfolgreich bestrittene Laufbahn des Athleten unterrichten. Bei altgedienten Athleten wurde der Ruhm noch gesteigert durch die Angabe der Ämter, die man in der Athletenvereinigung ${ }^{49}$ inne hatte, und durch Aufzählung der Bürgerrechte, die dem Athleten angetragen worden waren ${ }^{50}$.

Die Siegesverzeichnisse enthielten nicht selten allgemein gehaltene Aussagen über die Vielzahl der Siege und deren unterschiedlichen Charakter, wie etwa

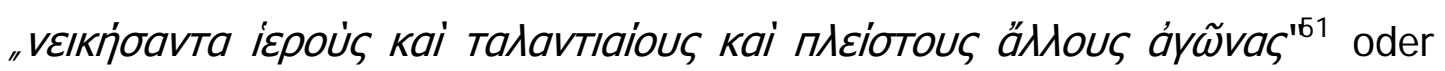

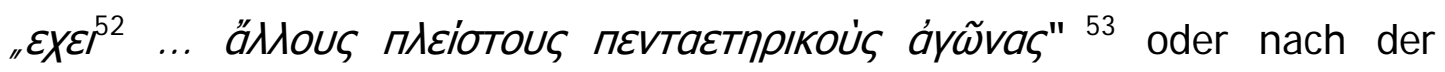
knappen Auflistung der Siege bei den Periodosspielen der Hinweis, dass die

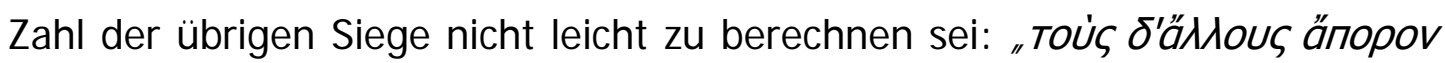

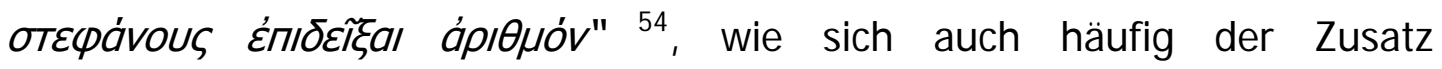

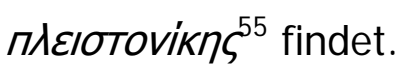

45 IG 2,1301; 7,4247; SEG 14,388; vgl. L. MORETTI, IAG, Nr. 22; 42; J. EBERT, Epigramme, Nr. 40; 70.

${ }^{46}$ P. Oxy. 2082; SEG 19, 397; 23, 325; vgl. L. MORETTI, IAG, Nr. 25; L. ROBERT, Lucillius, 192; J. EBERT, Epigramme, Nr. 39; 43.

47 Syll. 3, 69; IG 1, 2, 606; SEG 11, 290.

48 IVM 180 f.; vgl. L. MORETTI, IAG, Nr. 71; J. EBERT, Epigramme, Nr. 78.

${ }^{49} \mathrm{Vgl}$. L. MORETTI, IAG, Nr. 79; 84 . Vgl. H. W. PLEKET, Some Aspects of the History of the Athletic Guilds, in: ZPE 10, 1973, 220.

50 IG 14,1105 (Bürgerrecht von 16 Städten); vgl. L. ROBERT, in: RPh 4, 1930, 46 ff.; DERS., Anat. Stud. Buckler, 229 ff.; H. BUHMANN, Sieg, 113 f.

${ }^{51}$ Vgl. L. MORETTI, IAG, Nr. 72.

52 L. MORETTI, IAG, 184: ,... nella terminologia agonistica Ěx VIKãv."

53 IG 14, 747; IGR 1, 446; vgl. L. MORETTI, IAG, Nr. 68.

${ }^{54}$ Vgl. H. POMTOW, in: Klio 9, 1909, 183 ff.; L. MORETTI, IAG, Nr. 21; in: SEG 23, 325;

J. EBERT, Epigramme, Nr. 39. Ähnlich Pind. Nem. 2, 3 (über die Söhne des Timodemos):

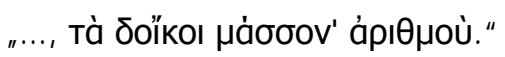


Die Siegeslisten konnten aber auch konkrete Zahlenangaben enthalten ${ }^{56}$, so etwa über die Zahl der Siege, die man bei ,Heiligen Spielen' als Knabe und

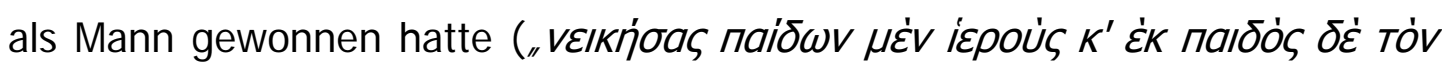

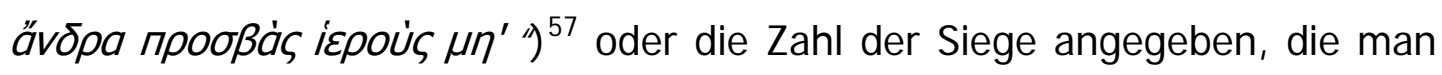

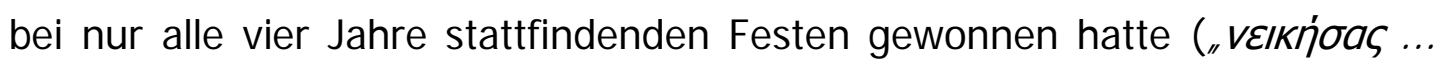

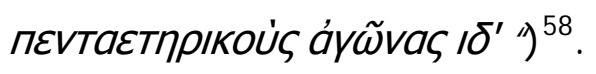

Das Pankration verzeichnet unter den hier abgehandelten schwerathletischen Disziplinen die meisten Periodoniken ${ }^{59}$. Doch auch aus dieser erlesenen Schar von gut einem Dutzend Athleten gelang es nur dem Dorieus aus der berühmten Diagoridenfamilie dreimal ${ }^{60}$, dieses zu erreichen. In Siegerepigrammen konnten dem Vermerk, dass man Periodonike war, der

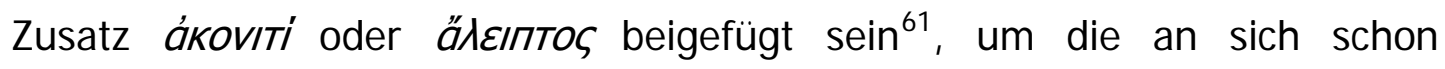
herausragende Leistung noch zu erhöhen. Die gleiche Absicht verfolgten

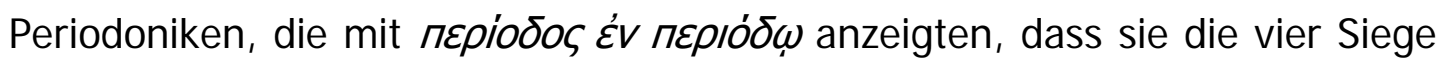
in dem kürzest möglichen Zeitraum erzielt hatten ${ }^{62}$.

55 IvO 55; vgl. L. MORETTI, IAG, Nr. 62; 72; 84; L. ROBERT, in: RA 1934, 59; DERS., in: Hellenica 5, 1948, 63.

56 Hatte man ursprünglich die Zahlen ausgeschrieben, so setzte man in der Kaiserzeit, spätestens seit dem 2. Jh. n. Chr. überwiegend Zahlzeichen; vgl L. MORETTI, IAG, 30.

57 IGR 4, 1519; vgl. L. MORETTI, IAG, Nr. 84.

58 IG 14, 746; IGR 1,445; vgl. L. MORETTI, IAG, Nr. 67.

${ }^{59}$ Vgl. R. KNAB, Periodoniken, Nr. 5, 10 f.; 13; 15; 17 f.; 21; 34 ff.; 40; 42; $45 ; 47$ f.; 51 f.; 64. Zu einem Pankratiasten, der sich als Periodonike rühmte, es aber aufgrund des Fehlens eines Sieges bei den Pythischen Spielen nicht sein konnte vgl. L. MORETTI, IAG, Nr. 71.

60 CIG 1715; Syll.3 82; vgl. R. KNAB, Periodoniken, 28 f. u. 57 f.; L. MORETTI, IAG, Nr. 23;W.Decker,Sport, 112.

61 Jul. Afric. Ol. 118; IG 14, 1104; IVM 199; vgl. R. KNAB, Periodoniken, Nr. 17 f.; 47; 64. Vgl. auch L. MORETTI, Olympionikai, Nr. 488, wo die Unkorrektkeit der Aussagen über den Pankratiasten Antenor von Milet besprochen wird.

62 IvO 231; CI G 2723; vgl. R. KNAB, Periodoniken, Nr. 35; 38. 
Auch priesen sich erfolgreiche Pankratiasten der Erst- und/oder Einmaligkeit inrer Siege oder Siegesserien, was sich auf einen geographisch oder ethnisch begrenzten Personenkreis ${ }^{63}$ wie auch durch die noch nie zuvor erbrachte Leistung auf die Gesamtheit der Athleten bzw. Menschen ${ }^{64}$ beziehen konnte.

Es zeigt sich, dass der Erfassung von Leistungen in den schwerathletischen Disziplinen Ringen, Boxen und Pankration große Beachtung zukam. Besonders in den Siegerepigrammen, die oft eine auffällig detailreiche Auflistung der Erfolge darbieten, wird die Absicht deutlich, die Erfolge der Athleten als einmalig darzustellen, z. T. in einer krampfhaften oder kuriosen Form der Suche nach Rekorden.

63 Paus. 6, 2, 1; 6, 13, 6; CIG 2810; Syll 3 274; vgl. R. KNAB, Periodoniken, 27; L. ROBERT, in: Hellenica 7, 1949, 105; DERS., Lucillius, 197; L. MORETTI, IAG, Nr. 29; 59; J. EBERT, Epigramme, Nr. 43.

64 IG 14, 746; IGR 1, 445; vgl. L. ROBERT, in: in: Hellenica 7, 1949, 105 f.; L. MORETTI, IAG, Nr. 67; J. EBERT, Epigramme, Nr. 67. 


\subsubsection{Leichtathletik}

Zu den hier behandelten leichtathletischen Disziplinen zählen wir den Sprung, den Speer- und den Diskuswurf, sowie die Laufkonkurrenzen und das Pentathlon.

\subsubsection{Sprung}

Bei der Untersuchung des Sprunges kommt im Gegensatz zum modernen Sport nur der Weitsprung in Betracht ${ }^{1}$. Für die Durchführung des Hochsprunges im Rahmen offizieller Wettkämpfe gibt es keine Zeugnisse ${ }^{2}$, und auch die wenigen Darstellungen einer Form des Stabhochsprunges geben keinen Hinweis auf eine wettkampfmäßige Durchführung ${ }^{3}$. Einen zählbaren Leistungsnachweis gab es jedoch auch bei anderen Sprungdisziplinen, wie das Beispiel eines spartanischen Sprungspieles lehrt, bei dem es galt, die Fersen gegen das Gesäß zu schlagen. ${ }^{4}$ Diese Übung wurde auch wettkampfmäßig betrieben, wobei die Zahl der Sprünge über den Sieg entschied. Pollux berichtet von einem Epigramm eines erfolgreichen

${ }^{1}$ Vgl. E. N. GARDINER, in: JHS 24, 1904, 180 f.; DERS., GASF, 296; H. A. HARRIS, GAA, 80; DERS., SGR, 35; J. JÜTHNER, AL 2, 159; R. PATRUCCO, Sport, 65; F. BREIN, GL 2, 105; M. I. FINLEY/H. W. PLEKET, Spiele, 75; D. C. YOUNG, Myth, 9, n. 3.

${ }^{2}$ Auch übte man im Rahmen der allgemeinen körperlichen Ertüchtigung Hochsprünge und Sprünge über Hindernisse; vgl. Sen. epist. 15; Lukian. Anach. 27; Veg. mil. 9. Die Verwendung eines Sprungbrettes (пе́таupov) finden wir nur bei Gauklern, vgl. E. N. GARDINER, in: JHS 24, 1904, 179; E. MEHL, Das Petauron - ein Federbrett, in: Mitt. d. Ver. kl. Phil. Wien 6, 1929, 28 ff.

3 Xen. de. re eq. 7,1, vgl. W. RUDOLPH, Antike Turngeräte, in: Klio 48, 1967, 87. Zu bildlichen Darstellungen (rotfigurige Vasenmalerei) vgl. z. B. B. SCHRÖDER, Sport, Taf. 83a; H. A. HARRIS, SGR, Taf. 29; (etruskisches Grabgemälde) F. WEEGE, in: J dl 31, 1916, Taf. 1; E. N. GARDINER, GASF, Taf. 174; DERS., AAW, Taf. 75; J. JÜTHNER, $A L$ 1, Taf. 24; F. BREIN, GL 2, Abb. 47; N. YALOURIS, Games, 186 f., Abb. 90. Ov. met. 8,366 und Anth. Pal. 9,533 erwähnen Stabsprünge bei venationes.

${ }^{4}$ Vgl. E. REISCH, in: RE 3 (1899), s. v. Bißßaбı, 390 f.; B. SCHRÖDER, Sport, 82; E. MEHL, in: RE 7, A2 (1948), s. v. Turnkunst, 254 f.; M. C. u. C. A. ROEBUCK, in: Hesperia 24, 1955, 158 ff. u. Taf. 63 f.; J. JÜTHNER, $A L$ 2, 161 n. 9; F. BREIN, GL 2,105 f. u. Abb. 30. 
Mädchens, das mit der Höchstleistung von tausend Sprüngen den Sieg

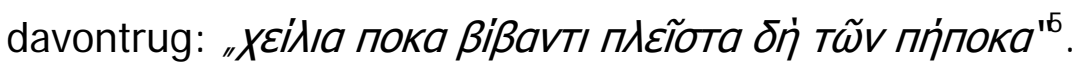

Der Weitsprung, der vorwiegend beim Fünfkampf durchgeführt wurde ${ }^{6}$ und dessen markanteste Übung war $^{7}$, ist durch umfangreiches Quellenmaterial belegt; dennoch konnte die Darstellung der antiken Sprungtechnik bis jetzt keine allseits befriedigende Lösung erfahren. Bei der Ausführung des Sprunges war den Athleten die Verwendung eigener Sprunggewichte

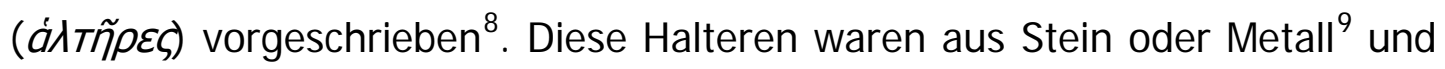
wiesen im Laufe der Zeit eine Veränderung ihrer Form auf - vom kolbenförmigen über den bauchigen bis hin zum zylindrischen Typ ${ }^{10}$ - wobei die Athleten im Wettkampf mit denselben Halteren antreten mussten. ${ }^{11}$ Die Halteren dienten dazu, eine möglichst große Sprungweite zu erzielen ${ }^{12}$ und bei der Landung einen sicheren Stand bzw. genauen Abdruck zu

\footnotetext{
5 Poll. 4,102.

${ }^{6}$ Vgl. L. MORETTI, IAG, 1 ff.; R. PATRUCCO, Sport, $65 \mathrm{ff}$.

${ }^{7}$ Vgl. E. N. GARDINER, GASF, 295; DERS., AAW, 144; W. W. HYDE, The Pentathlum J ump, in: AJ Ph 59, 1938, 406.
}

${ }^{8}$ Vgl. J. JÜTHNER, $A L$ 2, 162.

${ }^{9}$ Da Blei etwa viermal schwerer als Kalkstein ist, sind Halteren aus diesem Metall kleiner bzw. handfüllend; vgl. J. JÜTHNER, $A L$ 2, 182. Bei den starken Gewichtsunterschieden, die die erhaltenen Exemplare aufweisen, ist ein Durchschnitts- oder Idealgewicht nicht auszumachen; anders I. WEILER, Sport, 157.

10 Vgl. J. JÜTHNER, Turngeräthe, 3 ff.; DERS., Al 2, 162 ff.; E. N. GARDINER, in: JHS 24, 1904, 181; DERS., GASF, 298; DERS., AAW, 146; J. EBERT, Zum Pentathlon der Antike, Berlin 1963,46 ff.; R. PATRUCCO, Sport, 85 ff;St.G.Miller,Athletics, 115ff.

${ }^{11}$ Vgl. W. DECKER, Sport, 80.

12 Aristot. animal. incess. 3,705; vgl. F. FEDDE, Über den Fünfkampf der Hellenen, Leipzig 1889, 16 f.; J. JÜTHNER, Turngeräthe, 13; W. W. HYDE, in: AJPh 59, 1938, 409; N. YALOURIS, Games, 176. Über die dadurch veränderten physikalischen Verhältnisse vgl. E. LINDNER, Die Benutzung der Halteren im Weitsprung der Antike, AA 1956, 128 ff.; DERS., Sprung und Wurf, Schorndorf 1967, 44 ff.; J. EBERT, Pentathlon, 47. 
gewährleisten, da sonst der Sprung nicht gewertet wurde ${ }^{13}$. Der Sprung erfolgte von einer Sprungschwelle $(\beta a T r \dot{\rho})^{14}$, die als Ausgangspunkt der Weitenmessung diente und zur Vermeidung von Bevorteilungen regelgerecht betreten werden musste ${ }^{15}$. Gesprungen wurde in eine Sprunggrube ( бкá $\mu \mu a$,

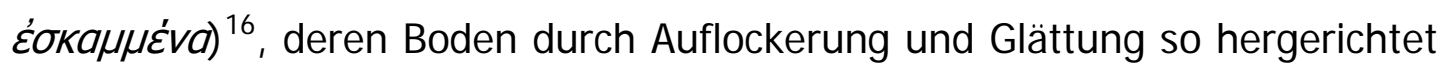
warr $^{17}$, dass der Fußabdruck des Springers gut erkenntlich war ${ }^{18}$. Ferner war die Sprunggrube auf eine Weite von 50 Fuß genormt ${ }^{19}$, was einer Sprungweite entsprach, die gewöhnlich von den Athleten nicht erreicht wurde $^{20}$. Die von den Springern erzielten Weiten wurden mittels einer in das „Aufgelockerte' gezogenen Linie markiert ${ }^{21}$. Das Ausmessen der Sprungweite $^{22}$ erfolgte mit Messlatten ${ }^{23}$, und zwar in dem üblichen

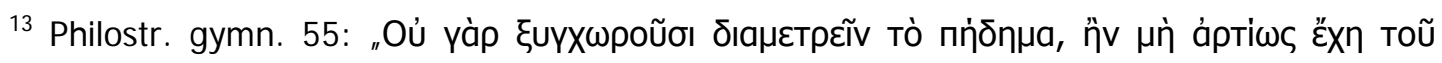
"xvouc."

14 Bekker Anecd. 224,12; vgl. E. N. GARDI NER, in: JHS 245, 1904, 74 ff.; J. JÜTHNER, $A L$ 2, 205 f. W. DECKER, Sport, 80.

15 Vgl. J. JÜTHNER, $A L$ 2, 207; R. PATRUCCO, Sport, $78 \mathrm{f}$.

16 Poll. 3,151; Zenob. 6,23; Schol., Plat. Krat. 413A; vgl. E. N. GARDINER, in: JHS 24, 1904, 70 ff.; J. EBERT, Pentathlon, 44; J. JÜTHNER, $A L$ 2, $202 \mathrm{ff}$.

${ }^{17}$ Vgl. M. HOMOLLE, in: BCH 23, 1899, 560 ff. u. $613 \mathrm{ff}$.

18 Philostr. gymn. 55; vgl. J. SWADDLING, Games, 54.

19 Zenob 6, 23; Lib, or. 69; Schol. Plat. Krat. 413A; Schol. Luc. somn. S. gall. 6; Suda s. v.

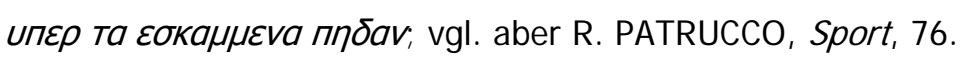

20 Vgl. J. EBERT, Pentathlon, 45. Anders W. W. HYDE, in: AJPh 59, 1938, 408.

${ }^{21}$ Schol. Pind. Nem. 5,19; Quint. Smyrn. 4,466; vgl. J.-H. KRAUSE, Gymnastik, Taf. 9, Abb. 24; J. JÜTHNER, Turngeräthe, Fig. 15; DERS., AL 1 Taf 20a; E. N. GARDINER, in: JHS 24, 1904, Fig. 4; DERS., GASF, Fig. 67, H. A. HARRIS, GAA, Fig. 106; R. PATRUCCO, Sport, Fig. 17; F. BREIN, GL 2, Abb. 31; V. OLIVOVÁ, Sport,115; E. N. GARDINER, GASF, 298; L. GRÜNDEL, in: Jdl 40, 1925, 88 und W. W. HYDE, in: AJPh 59, 1938, 413, halten Pfeile und Pflöcke zur Markierung der Weite für möglich; dagegen J. JÜTHNER, AL 2, 220 f., R. PATRUCCO, Sport, 77 ff u. J. SWADDLING, Games, 55.

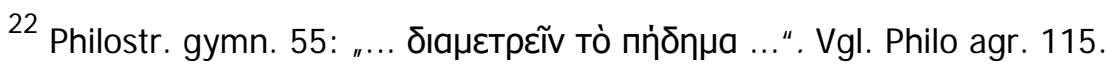

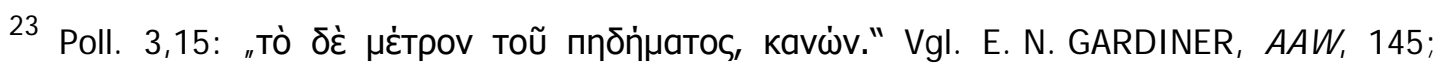

G. E. BEAN, in: AJA 60, 1956, 368; vgl. L. MORETTI, Un regolamento rodio per la gara del pentatlo, in: RFIC 34, 1956, 58: „Chiarissimo è ... l'accenno al kavóv ne quale deve 
Längenmaß, in $\mathrm{FuB}^{24}$. Was die erzielten Weiten anbelangt, so verfügen wir über konkrete Angaben ${ }^{25}$ zweier antiker Spizensportler. Dem spartanischen Olympioniken Chionis ${ }^{26}$, der auch ein ausgezeichneter Kurzstreckenläufer

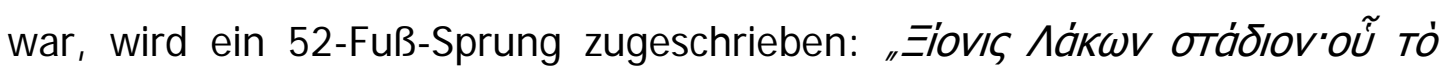

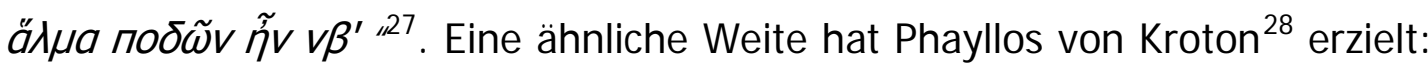

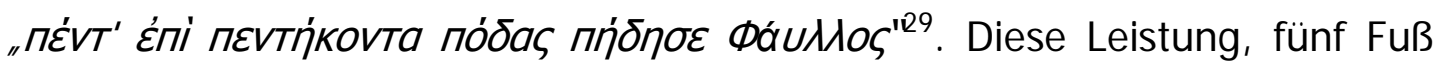
über die Sprunggrube hinaus, hat wegen ihrer Außergewöhnlichkeit schon die antiken Sportinteressierten begeistert ${ }^{30}$, fand entsprechend große

senz'altro riconoscersi l'unità di misura con cui i giudici calcolavano la lunghezza dei vari salti"; vgl. R. PATRUCCO, Sport, 90. Vgl. auch J. JÜTHNER, in: WS 53,1935, 72; DERS., $A L 2,220$, und J. EBERT, Pentathlon, 45, die unter kavóv das kanonische Maß für die Sprunggrube verstehen. Wenn J. JÜTHNER, in: WS 53, 1935, 72, bemerkt, dass es befremden müsste, wenn Pollux das Messen mit kavoves als Eigentümlichkeit der Sprungübung vermerkt, so ist dies mit den erheblich größeren Weiten bei den Wurfdisziplinen zu begründen.

${ }^{24}$ Eine wenn auch karikierte Variante des Messverfahrens bietet Aristophanes (nub. 144), wo die Distanz eines Flohsprunges mittels in Wachs modellierter Flohfüße ausgemessen wird.

${ }^{25} \mathrm{Zu}$ diesen beiden gesicherten Angaben ist nun vielleicht als ein weiteres Zeugnis, eine nur sehr fragmentarisch erhaltene delphische Inschrift eines 50-Fuß-Sprunges zu zählen: „Пદvтย́

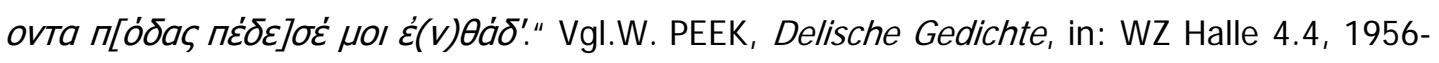
7, 572; J. EBERT, Pentathlon, 42 f.; J. JÜTHNER, $A L$ 2, 202 n. 121.

${ }^{26} \mathrm{Zu}$ Chionis und seinen sportlichen Leistungen vgl. L. MORETTI, Olympionikai, Nr. $42 \mathrm{ff}$. 27 Jul. Afric. Ol. 29.; vgl. E. N. GARDINER, in: JHS 24, 1904, 80; W. W. HYDE, in: AJ Ph 59, 1938,409; J. EBERT, Pentathlon, 39; R. PATRUCCO, Sport, 71.

${ }^{28}$ Vgl. H. E. STIER, in: RE 19,2 (1939), s. v. Phayllos 2, 1903 ff.; M. ROMANO, L'épigraphe ateniese à Phayllos (IG, I , 2, 823), in: ZPE 123, 1998, 105-116;

${ }^{29}$ Vgl. Th. PREGER, Inscriptiones Graecae metricae, Leipzig 1881, Nr. 142; vgl. E. N. GARDI NER, in: JHS 24, 1904, 70; W. W. HYDE, in: AJPh 59, 1938, 407; L. MORETTI, IAG, 27; J. EBERT, Pentathlon, 35; R. PATRUCCO, Sport, 70.

${ }^{30}$ Vgl. R. PATRUCCO, Sport, 69 n. I: „Sono queste due prove dell'interesse dei Greci per il record sportivo, e ciò costituisce un notevole punto d'incontro tra lo sporto greco e quello moderno." 
Resonanz bei den Scholiasten und Lexikographen ${ }^{31}$ und wurde auch später noch als Höchstleistung deklariert. ${ }^{32}$ Auch neuzeitliche Gelehrte haben wiederholt diese 55-Fuß-Weite als Rekordsprung betitelt ${ }^{33}$. Die Leistungen des Chionis und Phayllos haben aber auch unterschiedlichste Interpretationen erfahren, zumal eine Deutung der antiken Weitsprungtechnik sich stets konfrontiert sah mit diesen Sprungweiten. Die Leistung des Phayllos, die bei den Pythien erzielt wurde, ergibt nach Umrechnung mit dem dort wahrscheinlich zu Grunde gelegten salonischattischen Fuß $(0,296 \mathrm{~m})$ eine Weite von $16,28 \mathrm{~m}^{34}$. Die in Olympia erzielte Weite des Chionis könnte nach dem äginätisch-attischen Fuß (0,328 m) oder dem olympischen Fuß $(0,32045 \mathrm{~m})$ berechnet worden sein, was in jenem Falle eine Weite von 17,06 m, in diesem von 16,66 m ergibt ${ }^{35}$. Angesichts dieser Werte wurden die Sprungweiten als Erfindung ${ }^{36}$ und Verzerrung ohne Wahrheitsgehalt ${ }^{37}$, als fehlerhafte Übermittlung ${ }^{38}$ oder als reine

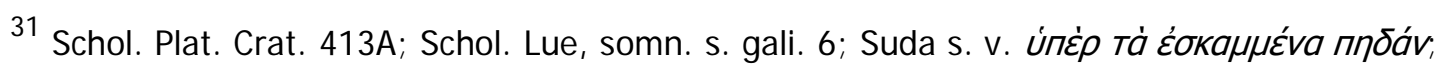
Eustath. Od. 8, 1591.

32 Zenob. 6,23.

33 Vgl. E. N. GARDINER, in: JHS 24, 1904, 70 ff., R. L. HOWLAND, Phayllos and the Long Jump Record, in: PCPhS 181, 1950-1, 30. Diese Autoren bestreiten allerdings trotz des Titels die Glaubwürdigkeit der Angabe. Ferner steht der Titel eines Rekordspringers eher dem Chionis zu, der ja nach Umrechnung in Meter (s. u.) eine absolut größere Weite erzielt hat.

34 Vgl. W DÖRPFELD, Beiträge zur antiken Metrologie, 1, Das solonisch-attische Maßsystem, in: MDAl (A) 7, 1882, 277 ff.; C. F. LEHMANN-HAUPT, in: RE 3 A2 (1929), s. v. Stadion (Metrologie), 919; J. EBERT, Pentathlon, 39.

${ }^{35}$ Vgl. W. DÖRPFELD, Beiträge zur antiken Metrologie, 5, Das äginäisch-attische Maßsystem, in: MDAI (A) 15, 1890, 167 ff.; E. N. GARDINER, Olympia. Its History and Remains, Oxford 1925, 235 n. l; J. EBERT, Pentathlon, 42.

${ }^{36}$ Vgl. z. B. E. N. GARDINER, $A A W, 152$ : "It is much more probable that the record is pure fiction."

37 Vgl. R. L. HOWLAND, in: PCPhS 181, 1950-1, 30: ,... the feet in which Phayllus's jump was measured may have been less than normal feet - perhaps the feet of his small son. We may like to assume that some wag composed the epigram for the amusement of those who knew what happened and the baffling of those who did not." 
Zahlenspielerei ${ }^{39}$ abgetan; andererseits ist bei Anerkennung der Richtigkeit der Angaben die Mehrzahl der Interpretationen bezüglich der Sprungtechnik zu verwerfen ${ }^{40}$. Die überzeugendste Lösung liefert J. EBERT, der an einen fünffachen Standsprung denkt, was der Gebrauch der Halteren und die im Pentathlon so bedeutungsvolle Zahl Fünf nahelegt ${ }^{41}$.

38 Die armenische Übersetzung der Eusebiuschronik hatte in ihrer lateinischen Übertragung (vgl. A. SCHOENE, Eusebi Chronicorum liber prior, Berlin 1875, 197) die Weitenangabe mächtig gedrückt: „Khionis Lakonius in stadio. Cuius unus saltus erat XXII cubitorum.“ Bei Gleichsetzung von cubitus und noúç wären 22 Fuß für einen einfachen Sprung im Bereich der menschlichen Leistungsfähigkeit, so dass man als Verschreibung für - hinstellte. Vgl. auch F. MEZÖ, Das Altertum 4,3, 1958, 170 ff.

${ }^{39}$ Vgl. E. N. GARDINER, in: JHS 24, 1904, 79: „It (i. e. the epigram) is artificial from beginning to end. Mark the alliteration, the constant repetition of number five, the symmetrical contrast between 50 feet +5 and 100 feet -5 ! ... But the artificiality is an argument against such a record ever having been made." B. SCHRÖDER, Sport, 144: „ ... Spielerei mit den Zahlen ... müßige Erfindung eines unbekannten Verseschmiedes ...“

40 Die Sprunghypothesen sind äußerst zahlreich, z. B. einfacher Tief-Weitsprung, einfacher Sprung mit Sprungbrett, verschiedenste Mehrfachsprünge mit und ohne Anlauf; vgl. die Zusammenstellung bei E. MEHL, in: RE 7 A2 (1948) s. v. Turnkunst, 2523 ff.; J. EBERT, Pentathlon, 54 ff.; H. A. HARRIS, GAA, 81. Auch neuere Lösungsvorschläge zum Weitsprung in der Antike (H. SCHMID, Der Weitsprung in der griechischen Antike. Eine Neubewertung unterschiedlicher Theorien unter Berücksichtigung eigener biomechanischer Versuche, Mainz 1997), die von einem Weitsprung mit Gewichten und einem Anlauf von etwa 30 m ausgehen oder (H. M. LEE, The Halma: A Running or Standing Jump?, in: G. P. SCHAUS u. a., Onward to the Olympics, Waterloo 2005, 153-163) einen "running long jump with weights" mit einem Anlauf von etwa zehn Metern annehmen, lassen noch Fragen offen.

${ }^{41}$ Vgl. J. EBERT, Pentathlon, 61 ff.; H. SCHOEBEL, Olympia, 75; M. I. FINLEY/H. W. PLEKET, Spiele, 75; W. DECKER, Sport, 81: „Trotz gewisser Unsicherheiten bei der Deutung etlicher bildlicher Darstellungen des Weitsprunges ist das von J. EBERT auch durch Versuche mit modernen Athleten flankierte Ergebnis überzeugend zu nennen. “ 


\subsubsection{Speerwurf}

Der Speerwurf konnte als Fernwurf ${ }^{1}$ und als Zielwurf zu Fuß ${ }^{2}$ und zu Pferde ${ }^{3}$ betrieben werden. Als Fernwurf - nur in sagenhaften Wettkämpfen als eigenständige Disziplin ausgetragen ${ }^{4}$ - war er lediglich als leichte Übung ${ }^{5}$ Bestandteil des Fünfkampfes ${ }^{6}$. Eine Untersuchung der sportlichen Leistungen im Speerwurf bezieht sich auf die Kriterien Weite und Zielgenauigkeit ${ }^{7}$.

Der im sportlichen Wettkampf benutzte Speer, der leichter als der Jagd- und Kriegsspeer war ${ }^{8}$, ist aus Holz gewesen ${ }^{9}$ und nach Ausweis der zahlreichen bildlichen Darstellungen etwa von Fingerdicke und 1,5 - 2 m Länge ${ }^{10}$. Die meisten dargestellten Speere sind stumpf und nur wenige eindeutig spit ${ }^{11}$,

\footnotetext{
${ }^{1}$ Pind. Pyth. 1,44; Isth. 2,35; Nem. 7,70; Bakchyl. 8,32; Lukian. Anach. 27; Hor. carm. 1,8, $10 \mathrm{ff}$.
}

2 Pind. Ol. 10,71; Nem. 9,55; Antiph. 2,2,7; Sil. Ital. 16, 567 ff.; Plut. Per. 36; Syll. 31059. Vgl. aber M. FABER, in: Philologus 50, 1891, 473 ff., der sich gänzlich gegen ein Zielwerfen ausspricht.

${ }^{3}$ Vgl. L. ZIEHEN, in: RE 18,3 (1949), s.v. Panathenaia, 479.

${ }^{4}$ Hom. II. 2,774; 24,884 ff., wobei Agamemnon den Sieg ohne Wettkampf davontrug.

${ }^{5}$ Vgl. I. WEI LER, Sport, 189.

${ }^{6}$ E. REISCH, in: RE 1,1 (1893), s. v. Akontion, 1184, und A. de RIDDER, in: DS 3,1 (1899), s. v. Jaculum, 601, sprechen fälschlicherweise beim Speerkampf im Fünfkampf von einer Kombination aus Weit- und Zielwurf.

${ }^{7}$ Sportliches Speerwerfen in die Höhe ist auszuschließen; vgl. E. N GARDINER, GASF, 352 u. 355.

${ }^{8}$ Tac. dial. 10; Lukian. Anach. 32.

${ }^{9}$ Bakchyl. 8,33; Theophr. hist. plant. 4,11,13.

10 Vgl. J. JÜTHNER, Turngeräthe, 36 f; DERS., AL 2, 309 ff.; E. N. GARDINER, GASF, 338; DERS., AAW, 169; F. MEZÖ, Geschichte, 127 f.; E. LINDNER, Sprung, 141; R. PATRUCCO, Sport, 172; I. WEILER, Sport, 166; W. DECKER, Sport, 81 (etwa Körperlänge).

${ }^{11}$ Vgl. J. JÜTHNER, Turngeräthe, Fig. 40 u. 42; DERS., AL 2, Fig. 70; E. N. GARDINER, in: JHS 27, 1907, Fig. 15; DERS., GASF, Fig. 105; DERS., AAW, Fig. 144; C. BLÜMEL, Spiel, Nr. 7; DERS., Hellenen, Nr. 111; R. PATRUCCO, Sport, Fig. 86 u. 89. 
z. T. mit langer Metallspitze ${ }^{12}$. Da die Szenen aber überwiegend dem Übungsbetrieb entnommen sind, ist es nahe liegend, dass man aus Sicherheitsgründen das Training damit bestritt ${ }^{13}$. Im Wettkampf werden spitze Speere verwendet worden sein, was nicht nur beim Zielwurf, sondern auch beim Weitwurf sinnvoll erscheint ${ }^{14}$.

Die Sportgeräte sind wohl vom Veranstalter gestellt worden ${ }^{15}$. Die Wurftechnik ist der unsrigen vergleichbar ${ }^{16}$, mit dem Unterschied, dass der

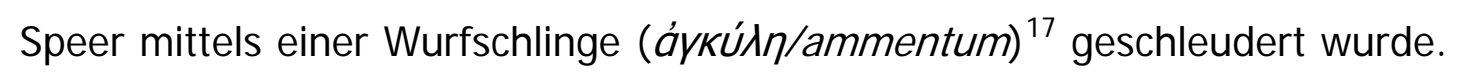
Wie viele Würfe der einzelne Wettkämpfer ausführte, ist unklar ${ }^{18}$. Da Athleten oft mit zwei Speeren dargestellt sind, könnte man auf zwei Würfe schließen ${ }^{19}$, der Siegpreis von drei Speeren legt einen dreifachen Wurf $\overline{12}$ Vgl. J. JÜTHNER, Turngeräthe, Fig. 21 f. u. 33a u. b; DERS., AL 2, Taf. 68 f.; F. MEZÖ, Geschichte, Fig. 65; C. BLÜMEL, Spiel, Nr. 40; DERS., Hellenen, Nr. 149; R. PATRUCCO, Sport, 49b.

13 Vgl. J. JÜTHNER, AL 2, 311; F. BREIN, GL 2, 109. M. POLIAKOFF, Terminology, 89, spricht von einer Schutzkappe, die beim Training an der Speerspitze angebracht wurde.

${ }^{14}$ Antiph. 2,2; vgl. M. FABER, in: Philologus 50, 1891, 471; H. A. HARRIS, Greek Javelin Throwing, in: G\&R 10, 1963, 27; DERS., GAA, 92; N. YALOURIS, Games, 196; H. SCHÖBEL, Olympia, 75; R. PATRUCCO, Sport, 172, denkt auch an die leichtere Überwindung des Luftwiderstandes. E. N. GARDINER, in: JHS 27, 1907, 250 und GASF, 339, irrt, wenn er stumpfe Speere für den Weitwurf, spitze für den Zielwurf annimmt.

${ }^{15}$ Es wird sich ähnlich verhalten haben wie mit den Disken, vgl. Paus. 6,19,4.

${ }^{16}$ Vgl. J. JÜTHNER, AL 2, 319 ff., R. PATRUCCO, Sport, 179. Zum Anlauf der Speerwerfer vgl. J. JÜTHNER, $A L$ 2, 344 ff., und H. A. HARRIS, GAA, Abb. 32, wo ein Speerwerfer seinen Anlauf ausmisst.

17 Vgl. A. v. DOMASZEWSKI, in: RE 1,2 (1894), s. v. Ammentum, 2901 f.; J. JÜTHNER, Turngeräthe, 39 ff.; DERS., AL 2, 315 ff., W. DECKER, Sport, 81.Zur Diskussion, ob die Ankyle fest am Speer angebracht war oder sich während des Fluges löste vgl. auch H. A. HARRIS, GAA, $93 \mathrm{f}$.

${ }^{18}$ Von nur einem Wurf zur Ermittlung des Siegers geht keiner der Gelehrten aus.

${ }^{19}$ Vgl. J. JÜTHNER, AL 2, 349 u. Taf. 63 u. 99, auch Taf. 90a (3 Speere), Taf. 75 (4 Speere) u. Taf. 94b (5 oder mehr Speere); R. PATRUCCO, Sport, 185 u. Figs. 93 u. 95; I. WEILER, Sport, 168. 
nahe ${ }^{20}$, und in Anlehnung an den Fünfkampf ist auch an fünf Versuche zu denken ${ }^{21}$. Der Wurf erfolgte von einer Abwurfstelle ${ }^{22}$, die nicht überschritten werden durfte ${ }^{23}$, und der Speer durfte aus Sicherheitsgründen einen seitlich begrenzten Sektor nicht verlassen ${ }^{24}$. Der Speer musste stecken bleiben ${ }^{25}$ oder zumindest einen deutlichen Einschlagspunkt hinterlassen, um korrektes Ausmessen der Wurfdistanz zu ermöglichen ${ }^{26}$. Die Wurfweite von der Abwurfstelle bis zum Auftreffpunkt wurde mit Hilfe von Messeinrichtungen, die im Stadion an den Längsseiten angebracht waren, ermittelt ${ }^{27}$. Der weiteste Wurf entschied über den Sieg $^{28}$.

20 Syll. 3,958; vgl. E. REISCH, in: RE 1,1 (1893), s. v. Akontion, 1184; A. de RIDDER, in: DS 3,1 (1899), s. v. Jaculum, 1194; F. MEZÖ, Geschichte, 129 f.; J. SWADDLING, Games, 54.

${ }^{21}$ Vgl. J. EBERT, Pentathlon, 13.

22 Vgl. E. N. GARDINER, GASF; 356; R. PATRUCCO, Sport, 180, u. N. YALOURIS, Games, 196, halten die Ablaufschranken im Stadion für die Abwurfstelle der Speerwerfer; dagegen J. JÜTHNER, $A L$ 2, $346 \mathrm{f}$.

23 Pind. Nem. 7, 70 ff.; Antiph. 2,2,3; vgl. J. JÜTHNER, Turngeräthe, 56 f.; DERS. (zu Pindar Nem. 7,70 ff.), in: WS 50, 1932, 166 ff.; C. A. FORBES, in: CJ 47, 1952, 173; E. D. FLOYD, Pindar's Oath to Sogenes, in: TAPhA 96, 1965, 139 ff.; R. PATRUCCO, Sport, $180 \mathrm{ff}$; H. M. LEE, The TEPMA and the Javelin in Pindar, Nemean 7,70-3, and Greek Athletics, in: JHS 96, 1976, $70 \mathrm{ff}$.

24 Vgl. E. N. GARDINER, GASF, 356; J. JÜTHNER, $A L$ 2, 346 f.; R. PATRUCCO, Sport, 180;W.Decker,Sport,81.

${ }^{25}$ Eur. Or. 1241; vgl. C. BLÜMEL, Hellenen, 83; H. A. HARRIS, in: G \& R 10, 1963, Fig. 1; R. PATRUCCO, Sport, Fig. 92, wo ein Athlet seinen Speer aus dem Boden zieht.

${ }^{26}$ Vgl. R. PATRUCCO, Sport, 184: “... il giavellotto si conficasse a terra o almeno vi lasciasse un segno evidente, per consentire la misurazione e il confronto con gli altri lanci dei concorrenti."

${ }^{27}$ Vgl. E. N. GARDINER, GASF, 356; DERS., AAW, 129; H. A. HARRIS, G\& R 10, 1963, 34 f.; E. K. BORTHWICK, The Gymnasium of Bromius, in: JHS 84, 1964, 50; R. PATRUCCO, Sport, 184.

28 Vgl. L. MORETTI, IAG, Nr. 75, wo ein Fünfkämpfer geehrt wird, der im Speerwurf unbesiegbar (а́парако́vтıбтоৎ) war. 
Bei den Menschen der griechisch-römischen Antike hat eine Vorstellung von einer durchschnittlichen Speerwurfleistung bestanden, da eine solche Weite als durchschnittliches Längenmaß benutzt wurde ${ }^{29}$. Eine konkrete Wurfweite ist uns nicht überliefert ${ }^{30}$. Wir haben aber eine Nachricht, die uns einen Anhaltspunkt über erzielte Speerwurfleistungen gibt. Statius sagt in seiner Beschreibung der Leichenspiele zu Nemea, dass die Pferderennbahn drei Bogenschuss- oder vier Speerwurfweiten betrage: „finem iacet inter utrumque, quale quater iaculo spatium, ter harudine vincas'is. Da beim Bogenschießen eine Weite von etwa 150 - $200 \mathrm{~m}$ als durchschnittliche Leistung angesehen werden kann ${ }^{32}$, müsste die Pferderennbahn etwa 450 $600 \mathrm{~m}$ Länge aufgewiesen haben, was sich in etwa mit den archäologischen Funden deckt ${ }^{33}$. Übertragen auf den Speerwurf ergäbe das eine Weite zwischen 100 und $150 \mathrm{~m}^{34}$. Da die antiken Sportspeere recht leicht waren etwa den heutigen Frauenspeeren vergleichbar - und durch die Verwendung der Ankyle eine Verlängerung des Impulsweges und damit eine erhöhte Abwurfgeschwindigkeit erreicht wurde ${ }^{35}$, liegt eine solche Weite absolut im Bereich des Möglichen.

29 Hom. II. 16, 589; Od. 23, 529; Hor. carm. 1,8,12: saepe trans finem iaculo nobilis expedito; vgl. M. FABER, in: Philologus 50, 1891, 477; J. JÜTHNER, $A L$ 2, 343; F. BREIN, GL 2, 110.

${ }^{30}$ Vgl. H. A. HARRIS, SGR, 37; I. WEI LER, Sport, 168.

${ }^{31}$ Stat. Theb. 4, $353 \mathrm{f}$.

32 Z. B. Strab. Mith. 14,1,23.

${ }^{33}$ Vgl. H. A. HARRIS, in: G \& R 10, 1963, 34; DERS., GAA, 95 ff.

34 Irrig M. VOGT, Der antike Sport, München 1934, 27, und E. MEHL, in: RE 7 A2 (148), s. v. Turnkunst, 2548, die beide von einer Laufstrecke und nicht von einer Pferderennbahn ausgehen und somit auf 46 bzw. $45 \mathrm{~m}$ kommen.

35 E. LINDNER, Sprung, $141 \mathrm{ff}$., hatte bei seinen Wurfversuchen mit Ankyle eine Steigerung bei etwa $70 \mathrm{~m}$ - Würfen um 10 m erreicht, was die Angaben J. JÜTHNERs, Turngeräthe, 54, von einer Steigerung der Wurfweite durch die Benutzung der Wurfschlinge um das Doppelte und die Annahme U. POPPLOWS, Leibesübungen und Leibeserziehung in der griechischen Antike, Schorndorf 19725, 155, dass die Wurfweite mittels Ankyle verdoppelt oder 
Was das Zielwerfen mit dem Speer anbelangt, so finden wir es literarisch wie inschriftlich erwähnt, jedoch ohne genaue Zielbeschreibung ${ }^{36}$. Diese liefern uns bedingt Vasenbilder, die alle ein Zielwerfen vom Pferd aus darstellen ${ }^{37}$. Als Ziel diente in den drei bekannten Darstellungen ein Schild, der an einem Pfosten erhöht aufgehängt war und dessen Zentrum ein Kranz bildete. Den bildlichen Darstellungen lässt sich aber nicht eindeutig entnehmen, wie der Sieger ermittelt wurde. Der Kranz im Zentrum legt ein sogenanntes InsSchwarze-Treffen nahe, wobei es offen bleibt, ob Treffer außerhalb des Zentrums gewertet wurden und ob es mehrere Versuche für den einzelnen Werfer gab und somit eine Art Punktwertung angewendet wurde ${ }^{38}$. Da auch jeweils mehrere z. T. zerbrochene Speere unter der Zielscheibe liegen, könnte man auch darauf schließen, dass schon das Steckenbleiben oder das Durchdringen des Schildes - was einen deutlich militärischen Bezug hätte ${ }^{39}$ bei der Siegerermittlung eine Rolle spielte. ${ }^{40}$

verdreifacht wurde, eindeutig widerlegt. Vgl. H. A. HARRIS, in: $G \& R$ 10, 1963, 30; DERS., GAA, 93 ff.; W. RUDOLPH, in: Klio 48, 1967, 81 n. 3.

${ }^{36}$ Es ist auch nicht bekannt, ob der Zielwurf zu Fuß mit Anlauf oder aus dem Stand erfolgte und wie groß die Distanz zum Ziel war. Beim Speerwurf zu Pferde scheinen die Wettkämpfer die Zielscheibe in vollem Galopp passiert zu haben.

37 Vgl. P. WOLTERS, Zu griechischen Agonen, Würzburg 1901, Taf. 1; B. SCHRÖDER, Sport, Abb. 37; S. REINACH, in: DS 3,1 (1900), s. v. Panathenaeen, Fig. 3752; E. N. GARDI NER, in: JHS 27, 1907, Taf. 20; DERS., GASF, Fig. 106; DERS., AAW, Fig. 12; R. PATRUCCO, Sport, Fig. 94; N. YALOURIS, Games, Fig. 105; F. BREIN, GL 2, Abb. 43; J. SWADDLING, Games, 53; V. OLIVOVÁ,Sport, 129.

38 Vgl. P. WOLTERS, Agone, 10 n. 14. Über die außergewöhnliche Treffsicherheit des Commodus berichtet Herod. I,15, 3 ff. Demzufolge soll der Kaiser in der Arena Tiere mit dem Speer erlegt haben, wobei er nie einen zweiten Wurf für dasselbe Tier brauchte und beliebig Herz oder Kopf treffen konnte.

39 Vgl. D. J. KYLE, Athletics in Ancient Athens, Leiden 1987, $186 \mathrm{f.}$

40 Vgl. E. REISCH, in: RE 1 (1894), s. v. Akontion, 1185; P. WOLTERS, Agone, 21; B. SCHRÖDER, Sport, 116 u. 140; L. ZIEHEN, in: RE 18,3 (1949), s. v. Panathenaia, 479; R. PATRUCCO, Sport, 187; I. WEI LER, Sport, 169. 


\subsubsection{Diskuswurf}

Der Diskuswurf ${ }^{1}$ wurde vorwiegend im Rahmen des Pentathlons ${ }^{2}$ und nur bei wenigen lokalen Veranstaltungen als eigenständige Disziplin durchgeführt ${ }^{3}$. Allgemein galt es, den Diskus ${ }^{4}$ möglichst weit zu werfen ${ }^{5}$; ein Hoch $^{6}$ - oder Zielwerfen mit dem Diskus als Wettkampfform ist nicht bekannt ${ }^{7}$.

Über die Wurfscheiben erhalten wir von Originalstücken ${ }^{8}$ und den bildlichen und plastischen Darstellungen ${ }^{9}$ mannigfaltige Anschauung. Erstaunt ist man dabei über die doch erheblichen Abweichungen, was Material, aber vor allem

1 Zum Ursprung des Diskuswurfes vgl. W. DECKER, in: Stadion 2, 1976, 196 ff. Wenig überzeugend L. CASTIGLIONE, Die Diskobolia - ein Agrarritus?, in: AAntHung 15, 1967, 409 ff.; M. K. LANGDON, Throwing the Discus in Antiquity. The Literary Evidence, in: Nikephoros 3, 1990, 177-182.

${ }^{2}$ Der Diskuswurf wurde nach Philostr. gymn. 3 zu den schweren Übungen des Fünfkampfes gezählt. Vgl. J. JÜTHNER, $A L$ 2, 256; R. PATRUCCO, Sport, 139.

${ }^{3}$ Philostr. gymn. 3; vgl. E. N. GARDINER, GASF, 337; L. MORETTI, IAG, 12; R. PATRUCCO, Sport, 138 f.; I. WEILER, Sport, 162.

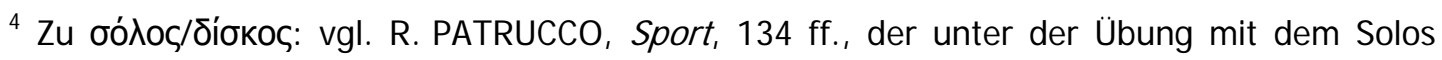
„moderno getto del peso“ versteht. Anders J. JÜTHNER, $A L$ 2, 225 ff., u. W. DECKER, in: Stadion 2, 1976, $203 \mathrm{f}$.

${ }^{5}$ Hom. II. 23, 843; 847; Od. 8, 189 ff.; Pind. Ol. 10, 72; Schol. Aristoph. Ach. 215; Lukian. Anach. 27; Philostr. her. 144, 32; Hor. carm 1,8,12; Stat. Theb. 6,70 ff.; Nonn. 27, 683 ff. ${ }^{6}$ Lukian. Anach. 27; DERS., dial. deor. 14,2; Stat. Theb. 6,681.

7 Poll. 8,72 berichtet von einem Sphalos-Spiel, bei dem ein an einem Seil befestigter Diskus (nach Art des Hammerwerfens) um die Wette geschleudert wurde; vgl. E. N. GARDINER, in: JHS 27, 1907, 5; DERS., GASF, 314; DERS., AAW, 154; J. JÜTHNER, $A L$ 2, 229 f.; R. PATRUCCO, Sport, 145 n. 3; N. YALOURIS, Games, 188; F. BREIN, GL 2, 108.

${ }^{8}$ Vgl. J. JÜTHNER, Turngeräthe, 18 ff.; DERS., $A L$ 2, 239 u. 241; E. N. GARDINER, in: JHS 27, 1907, 6; DERS., GASF, 316; DERS., AAW, 155.

9 Vgl. W. v. MASSOW, in: MDAl (A) 51, 1926, 44 f.; J. JÜTHNER, $A L$ 2, 261 ff.; ebd. 246 verweist Jüthner darauf, dass sich mit der Verwendung von Bronze statt Stein seit 500 v. Chr. nach Ausweis der Vasenbilder die Disken nicht im Umfang, sondern in der Dicke auf etwa ein Drittel verringerten. Vgl. auch DERS., Verzeichnete Athletendarstellungen auf Vasen, in: JÖAl 31, 1939, 6 ff. 
Gewicht, Durchmesser und Dicke der Wurfscheiben anbelangt ${ }^{10}$. Allein bei den in Olympia gefundenen Exemplaren bewegt sich der Durchmesser zwischen 17 bis $34 \mathrm{~cm}$ und das Gewicht von 1,268 bis $6,63 \mathrm{~kg}^{11}$, so dass Rückschlüsse auf ein Normgewicht ${ }^{12}$ anhand dieser Disken schwerlich möglich sind ${ }^{13}$. Es ist jedoch dabei zu bedenken, dass die erhaltenen Disken überwiegend Votivgaben waren ${ }^{14}$ und nicht im sportlichen Wettkampf Verwendung fanden ${ }^{15}$. Ferner lässt sich anführen, dass man beim Training mit unterschiedlichen Wurfscheiben übte ${ }^{16}$ und beim Wettkampf entsprechend der Altersklasse verschiedene Disken gebraucht wurden ${ }^{17}$. Sicher ist, dass - zumindest bei den großen Agonen $^{18}$ - die Teilnehmer einer Konkurrenz alle mit dem gleichen Diskus oder mit Wurfscheiben, die sich

${ }^{10}$ Vgl. I. WEI LER, Sport, 164.

11 Vgl. J. JÜTHNER, AL 2, 239 u. 241; H. A. HARRIS, GAA, 91, vermutet, dass die konservativen Olympier an den alten, schweren Disken festgehalten haben, wo andernorts schon leichtere verwandt wurden.

12 F. MEZÖ, Geschichte, 118 n. 6, vermerkt, dass das Gewicht der kleinsten und größten Sprunghantel fast genau übereinstimmt mit dem des kleinsten und größten Diskus.

13 Vgl. R. PATRUCCO, Sport, 141 f.; F. BREIN, GL 2, 108.

${ }^{14}$ Die beim Wettkampf gebrauchten Disken waren Eigentum des Veranstalters und blieben wie die Schilde für den Waffenlauf im Besitz der Ausrichter; vgl. J. JÜTHNER, $A L$ 2, 241; F. BREIN, GL 2, 108. Zu Disken, die als Weihegeschenke Verwendung fanden, vgl. L. E. ROLLER, in: Stadion 7, 1981, $3 \mathrm{f}$.

${ }^{15}$ Vgl. E. N. GARDI NER, GASF, 316, u. R. PATRUCCO, Sport, $141 \mathrm{f}$., halten den Diskus des Exoidas für den einzigen, der im Wettkampf Verwendung gefunden hat; anders J. JÜTHNER, $A L 2,239$.

${ }^{16}$ Vgl. B. SCHRÖDER, Sport, 118; J. JÜTHNER, in: RE 5,1 (1903), s. v. Diskos, 1188; DERS., Turngeräthe, 26; DERS., AL 2, 241.

17 Paus. 1,35,5.

${ }^{18}$ Philostr. her. 219 f. Bei der Vielzahl der lokalen Agone und dementsprechend lokaler unterschiedlicher Gewichtseinheiten muss man mit einer Vielzahl verschiedener Disken rechnen. Vgl. B. SCHRÖDER, Sport, 118; H. A. HARRIS, GAA, 86; R. PATRUCCO, Sport, 142 f.; vgl. auch E. MEHL, in: RE 7, A2 (1948), s. v. Turnkunst, 2526, der für die Wettkampfvorbereitung die Kenntnis des Gewichtes für wichtig erachtet. 
vollkommen glichen, warfen ${ }^{19}$ und dass es ein offizielles Normgewicht gab ${ }^{20}$. Der Diskuswurf ${ }^{21}$ erfolgte von einer Abwurfstelle $(\beta a \lambda \beta i)^{22}$, die aus Sicherheitsgründen vermutlich in der Mitte des Stadions $\operatorname{lag}^{23}$ und bei der Ausführung des Wurfes nicht überschritten werden durfte ${ }^{24}$. Fünf Versuche wird jeder Werfer gehabt haben. ${ }^{25}$ Anfangs wurde die Weite von der Abwurfstelle bis zu dem Punkt gemessen, an dem der Diskus zur Ruhe kam, später bis zu der Stelle, an der die Wurfscheibe zum ersten Mal aufkam²6. Der betreffende Punkt wurde mit einem Pflock ${ }^{27}$ markiert und dann

19 Paus. 6,19,4, vgl. aber E. N. GARDINER, GASF, 318; N. YALOURIS, Games, 188; H. SCHÖBEL, Olympia, 73; W. BACKHAUS, GL 2, 108; W. DECKER, Sport, 79.

20 Hom. Od. 8, 186 ff.; Philostr. her. 145,1 ff.; vgl. J. JÜTHNER, AL 2, 236 ff.; 244; W. DECKER, Sport, 96.

${ }^{21}$ Zu der vieldiskutierten Wurftechnik vgl. J. JÜTHNER, AL 2, 254 ff.; R. PATRUCCO, Sport, 151 ff.; J. SWADDLING, Games, 52.

22 Philostr. imag. 1,24; vgl. J. JÜTHNER, in: WS 53, 1935, 73 ff.; DERS., AL 2, 251 ff.; E. N. GARDINER, in: JHS 27, 1907, 9 f.; DERS., GASF, 318 f.; DERS., AAW, 155 ff., H. A. HARRIS, in: CR 75, 1961, 3 ff.; DERS.; GAA, 88; R. PATRUCCO, Sport, 147 ff.

${ }^{23}$ Vgl. R. PATRUCCO, Sport, 151, Fig. 56.

${ }^{24}$ Vgl. E. N. GARDINER, $A A W$, 157. Nicht zu belegen ist die Behauptung von H. SCHÖBEL, Olympia, 74, dass der Diskuswerfer ausschied, wenn er dreimal die Abwurflinie übertreten hatte.

25 Vgl. L. MORETTI, in: RFIC 34, 1956, 57; J. EBERT, Pentathlon, 13; R. PATRUCCO, Sport, 164; H. A. HARRIS, in: CR 88, 1974, 4 f., glaubt trotz der rhodischen Inschrift an drei Versuche.

${ }^{26}$ Vgl. J. JÜTHNER, AL 2, 260; I. WEI LER, Sport, 165.

${ }^{27}$ Hom. Od. 8,192; Stat. Theb. 6, 703; Eustath. Od. 8, 1591; vgl. J. JÜTHNER, Turngeräthe, 31 f. u. Fig. 27; DERS., $A L$ 2, 302 f. u. Abb. 65a u. Taf. 32b.;

B. SCHRÖDER, Sport, Taf. 60; E. N. GARDINER, in: JHS 27, 1907, Fig. 2; DERS., GASF, Fig. 74; DERS., AAW, Fig. 115; H. A. HARRIS, GAA, Fig. Ila; R. PATRUCCO, Sport, Fig. 74 ff.; M. I. FINLEY/H. W. PLEKET, Spiele, Abb. 33. 
ausgemessen $^{28}$. Zur Vermessung der Wurfweite dienten Messleisten, die an den Längsseiten des Stadions angebracht waren ${ }^{29}$.

Die durchschnittliche Diskuswurfweite galt in der Antike als allgemein bekanntes Längenmaß ${ }^{30}$. Die uns überlieferten Leistungen ${ }^{31}$ werden dieses Maß überschritten haben: Phlegias soll den Diskus über den Alpheios an seiner breitesten Stelle geworfen haben ${ }^{32}$. Genauer sind jedoch die Angaben des Heroen Protesilaos, der den Diskus, der zweimal so schwer war wie der olympische, $^{33}$ über 100 Ellen geworfen haben soll ${ }^{34}$. Die bekannteste Weitenangabe eines Diskuswurfes entnehmen wir dem berühmten Phayllos-

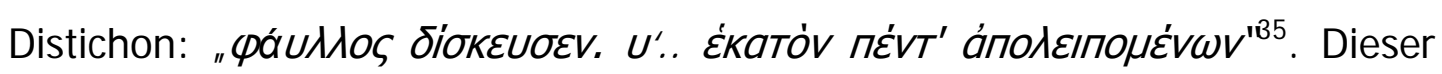

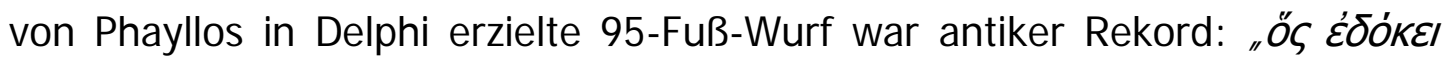

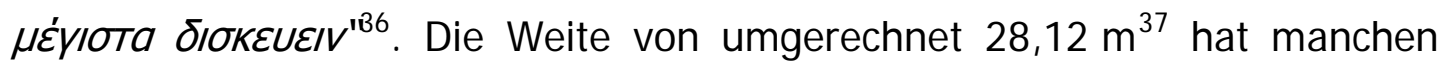

${ }^{28}$ Vgl. R. PATRUCCO, Sport, 134.

${ }^{29}$ Vgl. E. N. GARDINER, GASF, 319 f., Fig. 43; R. PATRUCCO, Sport, Fig. 56: „Indici fissi per la misurazione dei lanci"; DERS., Lo stadio di Epidauro, Florenz 1976, Fig. 39 f.

30 Hom. II. 2,774; 23, 826 ff.; Od. 4,626; 8,97 ff.; 17,168; Hor. carm. 1,8; vgl. E. N. GARDINER, in: JHS 27, 1907, 1; J. JÜTHNER, AL 2, 2565; L. CASTIGLIONE, in: AAntHung 15, 1967, 409; R. PATRUCCO, Sport, 133.

${ }^{31}$ W. DECKER, Sport, 79: „Absolute Wurfweiten sind nur zufällig überliefert, da es auf die hic et nunc erzielte Leistung ankam.“ Zu einem Fünfkämpfer, der im Diskuswurf unbesiegt

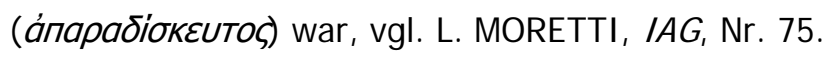

32 Stat. Theb. 6,668 ff.( Das entspräche einer Weite von 50 - $60 \mathrm{~m}$.)

33 Vgl. F. MEZÖ, Geschichte, 123 n. 22, der fälschlicherweise darunter eine Weitenangabe versteht, die doppelt so hoch ist, wie die in Olympia erzielte, und demzufolge die olympische Höchstleistung im Diskuswurf mit 24,03 m angibt.

34 Philostr. her. 3,8; vgl. H. A. HARRIS, GAA, 9; K. KRAMER, Studien, 51.

${ }^{35}$ Vgl. J. JÜTHNER, $A L$ 2, 261; R. PATRUCCO, Sport, 168.

${ }^{36}$ Zenob. 3, 23; vgl. R. PATRUCCO, Sport, 168.

37 Davon ausgehend, dass die Weite nach dem solonisch-attischen Fuß $(0,296 \mathrm{~m})$ ausgemessen wurde, vgl. J. EBERT, Pentathlon, 35. 
Zweifel an der Glaubwürdigkeit der Aussage geweckt ${ }^{38}$. Dieser ist aber insofern unbegründet, da wir nicht die technischen Daten des Sportgerätes kennen, womit auch ein Vergleich mit heutigen Leistungen unsinnig erscheint ${ }^{39}$. Bei der technischen Ausführung des Diskuswurfs werden zwei Varianten bevorzugt:ganze Körperdrehung oder Wurf aus der Haltung, wie sie uns der Diskobulus des Myron zeigt. ${ }^{40}$

Man kann festhalten, dass die erzielten Weiten in den drei technischen Disziplinen, die meistens nur als Teildisziplinen des Pentathlons stattfanden, ausgemessen wurden. Besonderer Erwähnung wert waren Weiten, die sich von durchschnittlichen Leistungen abhoben oder gar Rekorde darstellten.

\footnotetext{
${ }^{38}$ Vgl. H. A. HARRIS, in: G \& R 7, 1960, 8, glaubt, dass der Epigrammatiker aufzeigen wollte, dass Phayllos ein ausgezeichneter Weitspringer, aber ein dürftiger Diskuswerfer war, und spricht inm nur ein ,junior schoolboy's Standard in the discus“ zu; vgl. DERS., GAA, 91.

39 J. JÜTHNER, $A L$ 2, 246, hat durch Vergleich mit modernen Wurfleistungen den von Phayllos benutzten Diskus auf etwa $4 \mathrm{~kg}$ geschätzt. E. MEHL, in: RE 7 A2 (1984), s. v. Turnkunst, 2548, spricht von einer $5 \mathrm{~kg}$ schweren Wurfscheibe.
}

${ }^{40}$ Vgl. J. J ÜTHNER, $A L$ 2, 267 ff.; R. PATRUCCO, Sport, 152 ff; W. DECKER, Sport,79f. 


\subsubsection{Lauf}

Der Wettlauf $^{1}$ gehörte zu den ältesten und am meisten geschätzten Disziplinen des antiken Sports ${ }^{2}$. Die zahlreichen Laufkonkurrenzen unterschieden sich in erster Linie durch die Distanz - was sich auch deutlich in den verschiedenen Laufstilen zeigt $^{3}$ - und die bei bestimmten Konkurrenzen benötigten Utensilien ${ }^{4}$. Als Grundlage für die einzelnen Laufdisziplinen galt die Strecke eines Stadions ${ }^{5}$. Für den Kurzstreckenlauf

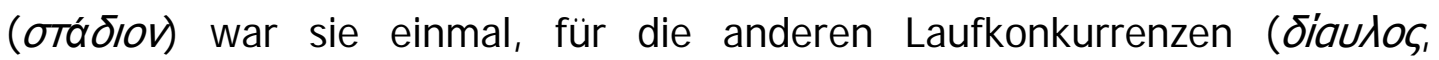

1 Nicht berücksichtigt werden hier außergewöhnliche Laufwettbewerbe, die im Zusammenhang mit speziellen lokalen Festlichkeiten stattfanden. Zum spartanischen Weintraubenlauf, einem Lauf mit gefülten Wasserkrügen bei den De/phinien u. ä., vgl. L. DEUBNER, Attische Feste, Hildesheim 1959², 143 ff.; H. JACHOWSKI, in: Leibeserziehung 10, 1963, 283; K. KRAMER, Studien, 28; N. YALOURIS, Games, 36; I. WEILER, Sport, 146 f.

2 Vgl. H. JACHOWSKI, in: Leibeserziehung 10, 1963, 281 u. 283 ff.; zum Stellenwert der einzelnen Laufdisziplinen vgl. L. ZEEHEN, in: RE 18,3 (1949), s. v. Panathenaia, 476 f.; H. A. HARRIS, GAA, 65; J. EBERT, in: WZ Halle 15, 1966; 3, 375 ff.; J. JÜTHNER, $A L$ 2, 36; H. BUHMANN, Sieg, 51. Zum Laufsport der Frauen Plat. leg. 8,833c-e; Theokr. 18, 22; Paus., 3,13,7; Philostr. gymn. 27; vgl. R. PATRUCCO, Sport, 100 f.; T. F. SCANI ON, The Footrace of the Heraia at Olympia, in: AncW 9, 1984, 77 ff.; DERS. The Heraia at Olympia Revisited, in: Nikephoros 21, 2008, 159-196.

3 Philostr. gymn. 32 f.; vgl. L. GRÜNDEL, Die Darstellung des Laufes in der griechischen Kunst, Würzburg 1934; J. JÜTHNER, AL 2, 22 ff.; F. BREIN, GL 2, 102.

${ }^{4}$ Ob die Antike den Hürdenlauf kannte, bleibt fraglich. Vgl. B. SCHRÖDER, Sport, 106 u. Abb. 24 f.; F. MEZÖ, Geschichte, 69 u. Abb. 31; E. MEHL, in: RE 7, 2 (1948), s. v. Turnkunst, 2521; R. PATRUCCO, Sport, 129 u. Fig. 45 f. Gegen Hürdenlauf sprechen sich M. I. FINLEY/ H. W. PLEKET, Spiele, 75, aus.

5 Zu den unterschiedlichen Stadionlängen vgl. W. ZSCHIETZSCHMANN, Wettkampf- und Übungsstätten in Griechenland 1, Schondorf 1960, 7 ff.; F. KRINZINGER, Untersuchungen zur Entwicklungsgeschichte des griechischen Stadions, Diss. Innsbruck 1968, 18 ff.; O. BRONEER, Isthmia I, Princeton 1978, 174 ff.; S. G. MILLER, in: Hesperia 48, 1979, 95; C. K. WILLIAMS/P. RUSSELL, Corinth Excavations of 1980, in: Hesperia 50, 1981, $1 \mathrm{ff.;}$ D. G. ROMANO, The Ancient Stadium. Athletics and Arete, in: AncW 7, 1983, $9 \mathrm{ff}$. 


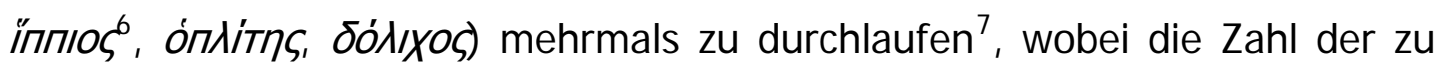
absolvierenden Stadien nur im Langlauf (zwischen 7 und 24 Stadien) bei den verschiedenen Veranstaltungen differierte ${ }^{8}$. Abweichend von diesem Maßsystem fand z. B. der Waffenlauf von Plataiai statt ${ }^{9}$ sowie die Fackelläufe (Аанпабпброніa), die nicht in einer offiziellen Wettkampfstätte, sondern meistens zwischen zwei Altären ausgetragen wurden ${ }^{10}$, wobei am Zielaltar das Opfer angezündet werden musste ${ }^{11}$. Für die im Stadion stattfindenden Laufkonkurrenzen gab es verschiedene Startvorrichtungen, z.T. feinst ausgeklügelte Systeme ${ }^{12}$, die die Chancengleicheit wahrten ${ }^{13}$ bzw. einen

6 J. SWADDLING, Games, 45, gibt statt vier Stadien fälschlicherweise sechs Stadien für den Hippios an.

7 Vgl. J. JÜTHNER, $A L$ 2, 95 ff.; vgl. ebd. 105 n. 220 zu anderen außergewöhnlichen Laufdistanzen auf der Basis des Stadionmaßes.

8 Vgl. J. JÜTHNER, in: RE 5,1 (1903), s. v. dolichos 4, 1282 f.; DERS., AL 2, 108 f.; L. MORETTI, IAG, 20; H. A. HARRIS, GAA, 73; R. PATRUCCO, Sport, 95 f.; J. EBERT, in: Stadion 5,1, 1979, 16. Die Vermutung von J. JÜTHNER, in: RE 5,1 (1903), s. v. dolichos 4 , 1282, und B. SCHRÖDER, Sport, 104, dass die Strecke des Dolichos je nach den Verhältnissen des Ortes und den Fähigkeiten der gemeldeten Bewerber bemessen wurde, hat schon F. MEZÖ, Geschichte, 74, zurückgewiesen.

${ }^{9}$ Der Waffenlauf wurde über eine Distanz von zwei Stadien (Aristoph. av. 292; Poll. 3,151; Paus. 2, 11, 8), in Nemea über vier Stadien (Philostr. gymn. 7), in Plataiai über 15 Stadien (Paus 9,2,6; Philostr. gymn. 8) ausgetragen. Vgl. L. ROBERT, in: REA 31, 1929, 15; A. MITSOS, in: MDAl (A) 65, 1940, 51; J. JÜTHNER, AL 2, 124 n. 283; G. NEUMANN, Der Waffenlauf im antiken Griechenland, in: U. HAUSMANN (Hrsg.), Der Tübinger Waffenläufer (Tübinger Studien zur Archäologie und Kunstgeschichte 4, 1977), 38; W. DECKER, Sport, 62.

10 Schol. Aristoph. ran. 131; vesp. 1203; Paus, 1,30,2. Davon abweichend Plaut. Sol. 1,4; Plat. Phaidr. 231.

${ }^{11}$ Das setzte natürlich voraus, dass man mit brennender Fackel den Zelaltar erreichte, andernfalls auch nicht siegen konnte( Paus. 1,30,2.).

${ }^{12}$ Vgl. O. BRONEER, in: Hesperia 27, 1958, 10 ff.; DERS., in: Klio 39, 1962, 262 ff.; DERS., in: AJA 76, 1972, 205 ff.; H. A. HARRIS, GAA, 67 ff.; DERS., SGR, 27 ff.; DERS., in: G\& R 7, 1960, 25 ff.; J. JÜTHNER, $A L$ 2, 51 ff.; F. KRINZI NGER, Stadion, 204 ff. ;B. RIEGER, Von der Linie (gramme)zur Hysplex. Startvorrichtungen in den panhellenischen Stadien Grichenlands 
Frühstart ${ }^{14}$ verhindern sollten. Bei Läufen über mehr als ein Stadion hatten die Teilnehmer einmal bzw. mehrmals ein Wendemal (канпти́p)zu umlaufen $^{15}$. Der Sieger wurde beim Überqueren der Ziellinie ${ }^{16}$ durch die Kampfrichter ${ }^{17}$, die auch auf die korrekte Durchführung des Wettkampfes achteten ${ }^{18}$, mittels Augenschein bestimmt ${ }^{19}$.

Seit dem 6. Jh. v. Chr. liefen die Wettkämpfer gewöhnlich unbekleidet ${ }^{20}$ und ohne Schuhzeug ${ }^{21}$. Für den Waffenlauf, bei dem eine schrittweise

(Nikephoros Beihefte 9) Zum Startzeichen vgl. H. A. HARRIS, GAA, 66; J. JÜTHNER, $A L 2$, 90.

13 Vgl. R. PATRUCCO, Sport, 110.

${ }^{14}$ Nach Herodot 8,59 wurde der Frühstart mit Auspeitschen bestraft. Zum Startvorgang vgl. P. ROOS, The Start of the Greek Foot Race, in: OAth 6, 1965, 149 ff.;W. DECKER, Sport, 59. 15 Über das Problem eines einzigen Wendemals oder zahlreicher individueller Wendemarken vgl. H. A. HARRIS, in: G\&R 7, 1960, 33 f.; DERS., GAA, 31 f.; J. JÜTHNER, $A L$ 2, 104 f.; R. PATRUCCO, Sport, 106 ff.; S. G. MILLER, in: AJA 84, 1980, 159 ff.; I. WEILER, Sport, 151 f.; B. RIEGER, Hysplex, 49 ff.

${ }^{16}$ Vgl. B. SCHRÖDER, Sport, 106; I. WEI LER, Sport, 150.

17 Paus. 6,3,1 berichtet von einem Zwischenfall bei den Ol. Sp. 96 (= 396 v. Chr.), bei dem von den drei Hellanodiken zwei dem Läufer Eupolemos aus Elis den Sieg zugesprochen hatten, ein Kampfrichter aber den Leon von Ambrakia vorn gesehen hatte. Vgl. F. MEZÖ, Geschichte, 70; L. MORETTI, Olympionikai, Nr. 367; H. BUHMANN, Sieg, 12.

${ }^{18}$ Zu den bei den Laufkonkurrenzen zu beachtenden Regeln vgl. J. JÜTHNER, $A L$ 2, 87 f.; M. I. FINLEY/H. W. PLEKET, Spiele, 80. Zu Unsportlichkeiten bei Wettläufen vgl. C. A. FORBES, in: CJ 47; 1952, 172 f.; J. SWADDLING, Games, 48.

19 Eine Art Zelband war den Alten fremd; vgl. B. SCHRÖDER, Sport, 106; vgl. auch R. MERKELBACH, in: ZPE 11, 1973, 129.

20 In homerischer Zeit (II. 23,683) lief man noch bekleidet. Von einem nicht genau bestimmbaren Zeitpunkt an liefen die Athleten nackt. Vgl. J. JÜTHNER, $A L$ 2, 48 ff.; J. SWADDLING, Games, 49; I. WEILER, Sport, 116 f.; vgl. auch die haltlosen Spekulationen von J. A. ARIETI, in: CW 68, 1975, 431 ff. Zu den Läufern mit Perizoma vgl. J. JÜTHNER, $A L$ 2, Abb. 2 u. 4; R. PATRUCCO, Sport, Fig. 24. 
Reduzierung der Ausrüstung Helm und Beinschienen verschwinden ließ ${ }^{22}$, galt der Schild später als Erkennungszeichen dieser außergewöhnlichen Laufkonkurrenz ${ }^{23}$. Diese Schilde müssen (zumindest bei bedeutenden Wettkämpfen) genormt gewesen sein, was die Aufbewahrung von 25 identischen Schilden im Schatzhaus zu Olympia nahe legt ${ }^{24}$. Die bei der ৯анпабпброніa verwendeten Fackeln hatten nach Ausweis der bildlichen Zeugnisse in etwa die gleiche Gestalt ${ }^{25}$. Wie bei den anderen Konkurrenzen waren auch die Teilnehmer der verschiedenen Laufdisziplinen entsprechend ihres Alters in Klassen unterteilt ${ }^{26}$, wobei häufig der Langlauf ${ }^{27}$ und der Waffenlauf ausschließlich den Männern vorbehalten blieben ${ }^{28}$. Für den Fackellauf ${ }^{29}$, der als Einzellauf ${ }^{30}$ wie auch als Stafettenlauf ausgetragen

21 Gegen Poll. 3,155. Vgl. H. J. KRAUSE, Gymnastik, 362; E. N. GARDINER, AAW, 273; J. JÜTHNER, $A L$ 2, 47; I. WEILER, Sport, 149. Nach Strabo $(11,5,6)$ kannte die Antike schon Spikes, die man im Kaukasus bei Eis und Schnee trug; vgl. H. A. HARRIS, GAA, 66.

22 Pind. Ol. 4, 22; Pyth. 9, 1; 10,13 f.; Isth. 1,23; Philostr. gymn. 8; Paus. 2,11,8; 6,10,4. Vgl. F. HAUSER, in: Jdl 10, 1895, 195 ff.; G. v. BRAUCHITSCH, Die panathenäischen Preisamphoren, Leipzig 1910, 137 f.; W. W. HYDE, Olympic Victor Monuments, Washington 1921, 161 ff.; J. D. BEAZLEY, in: ABSA 46, 1951.; G. NEUMANN, Waffenlauf, 37 ff.

${ }^{23}$ Heliodor $(4,3)$ lässt noch im 3. Jh. n. Chr. seinen Helden Theagenes bei den pythischen Spielen die volle Rüstung anlegen. Zur Unterscheidung bildlicher Darstellungen des Waffenlaufs von militärischen Szenen vgl. J. JÜTHNER, $A L$ 2, 132; F. BREIN, GL 2, 104; I. WEI LER, Sport, 154.

${ }^{24}$ Paus. 5,12,8. Vgl. J. JÜTHNER, $A L$ 2, 123; G. NEUMANN, Waffenlauf, 38; J. SWADDLING, Games, 44.

${ }^{25}$ Vgl. J. JÜTHNER, $A L$ 2, 137 ff. Zum Kopfschmuck der Waffenläufer vgl. ebd. $141 \mathrm{f}$.

${ }^{26}$ Vgl. E. N. GARDINER, GASF, $271 \mathrm{ff}$. Zu unterschiedlichen Distanzen bei verschiedenen Altersklassen bzw. weiblichen Teilnehmern vgl. Plat. leg. 8,833c-d; Paus. 5,16,2 f.; vgl. auch R. PATRUCCO, Sport, $100 \mathrm{f}$.

27 Vgl. Th. KLEE, Agone, 27 ff. u. 51; J. EBERT, in: Stadion 5.1, 1979, 16. Vgl. aber Paus. $10,7,5$.

28 Vgl. L. MORETTI, IAG, 128; J. JÜTHNER, $A L$ 2, 115; L. ROBERT, in: RA 1978, 279; J. EBERT, in: Stadion 5.1, 1979, 14.

${ }^{29}$ Man kannte auch Fackelwettrennen zu Pferde; Plat. rep. 1,328a; IG 9,2,528 u. 531. Vgl. E. N. GARDINER, AAW, 143 u. Taf. 35,1; J. JÜTHNER, in: RE 12,1 (1924), s. v. 
wurde $^{31}$, lässt sich nicht genau bestimmen, wie viele Läufer ein Team bildeten ${ }^{32}$. Sonst war die Zahl der Läufer pro Rennen durch die Zahl der Startplätze ${ }^{33}$ oder Ausrüstungsgegenstände ${ }^{34}$ beschränkt, so dass bei einer größeren Zahl gemeldeter Teilnehmer ${ }^{35}$ Vorläufe ausgetragen wurden ${ }^{36}$. Die Startplätze wurden ausgelost ${ }^{37}$.

Die Reihe der Hinweise auf die Leistung setzte bereits bei einem guten Abschneiden $e^{38}{ }^{38}$ und ein Unentschieden fand schon entsprechende Anerkennung ${ }^{39}$. Ein Sieg, der nicht durch ein Freilos begünstigt worden war,

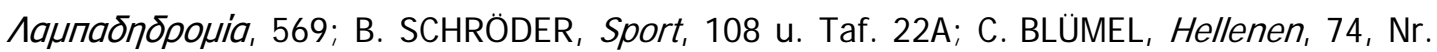
70.

30 Vgl. J. R. SITLINGTON STERRETT, in: AJPh 22, 1901, 406; J. EBERT, in: Stadion 5.1, 1979, $10 \mathrm{ff}$.

31 IG 2 2/3,956 (Palästren stellen Mannschaften); IG 2 2/3,2311 u. 3019 (Phylen stellen Mannschaften).

${ }^{32}$ Vgl. R. PATRUCCO, Sport, 127 ff.; J. SWADDLING, Games, 45.

33 Das Stadion in Epidauros war für 11, das von Delphi für 17 oder 18, das von Olympia für 20 Läufer eingerichtet, vgl. C. GASPAR, in: DS 4.1 (1907), s.v. Olympia, 176; E. N. GARDINER, GASF, 253; DERS., Olympia, 285; F. KRINZINGER, Stadion, $204 \mathrm{ff}$. et passim; R. PATRUCCO, Sport, $103 \mathrm{f}$; ; I. WEILER, Sport, $148 \mathrm{f}$.

${ }^{34}$ So etwa durch die Anzahl der genormten Schilde für den Waffenlauf in Olympia.

35 Bei Xenophon (anab. 4,27) treten 60 Kreter zum Langlauf an. Gewöhnlich werden es aber weniger gewesen sein; z. B. Anth. Pal. 6,259 erwähnt neun Läufer; bei Strabo (geogr. 6, 1, 12) waren es wenigstens acht Teilnehmer; vgl. J. JÜTHNER, $A L$ 2, 86; R. PATRUCCO, Sport, 201.

${ }^{36}$ Paus. 6,13,4. Vgl. I. WEI LER, Sport, 150. Nach E. N. GARDINER, in: JHS 23, 1903, 266 f., gab es keine Qualifikationsrennen beim Langlauf.

37 Heliod. ait. 4,3. Vgl. F. MEZÖ, Geschichte, 69; E. N.GARDI NER, AAW, 277 f.; J. JÜTHNER, $A L 2,87$.

38 Vgl. G. RADET, Inscription de la région du Méandre, in: $\mathrm{BCH}$ 10, 1890, 239 n.15;

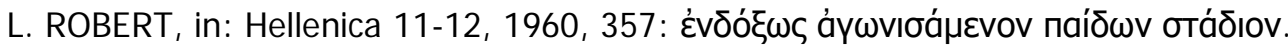

${ }^{39}$ Vgl. M. GOUGH, in: Anat. Stud. 2, 1952, 127; in: SEG 12, 1955, 112; L. MORETTI, IAG, Nr. 86; J. EBERT, Pentathlon, 10. Vgl. F. MEZÖ, Geschichte, 205 n. 18; H. A. HARRIS, GAA, 126;

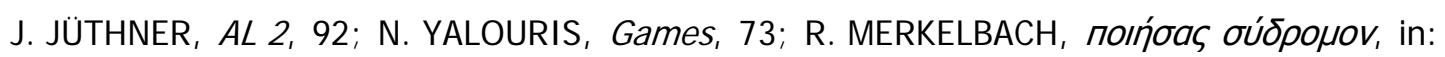


wurde herausgehoben $^{40}$. Prächtige Siege wurden besonders vermerkt ${ }^{41}$, und

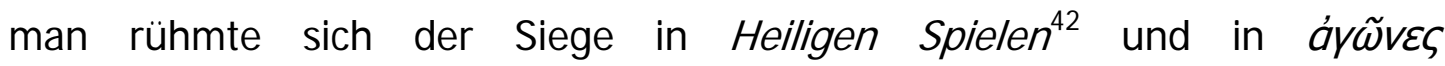

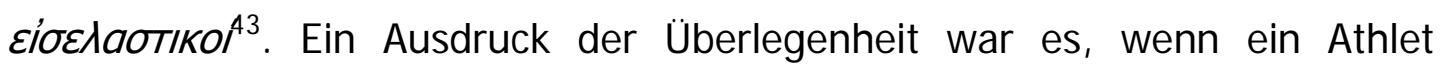
ákovití gewann ${ }^{44}$ bzw. die Gegner zurücktraten, nachdem sich der vermeintlich hoch überlegene Favorit in die Teilnehmerliste eingeschrieben

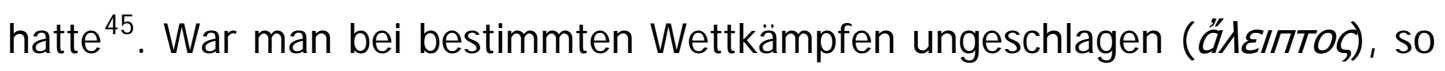
wurde dies herausgestrichen ${ }^{46}$.

Als Zeichen der Vielseitigkeit galt es, nicht nur in verschiedenen Laufkonkurrenzen zu glänzen ${ }^{47}$, sondern auch in anderen Disziplinen erfolgreich zu sein. So kennen wir siegreiche Läufer, die auch in

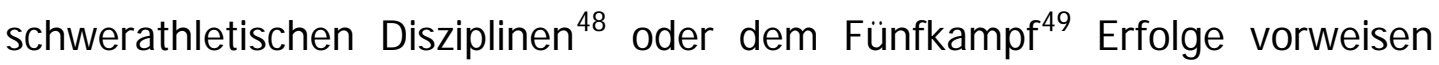
konnten. Gewann man bei einer Veranstaltung zwei Laufentscheidungen -

ZPE 11, 1973, 128 ff. Vgl. auch Herod. 5,22; Sen. epist. 83. Ferner CIL 6, 33950=ILS 5278: „una palma rev(ocatus)".

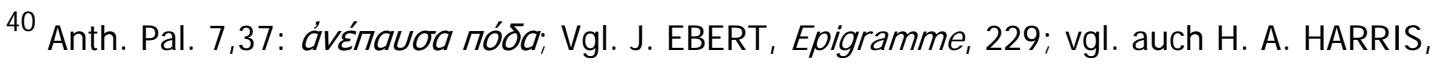
GAA, 66; J. JÜTHNER, $A L$ 2, 99; zweifelnd E. N. GARDINER, in: JHS 23, 1903, 267 n. 33.

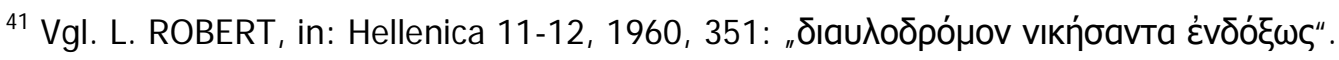

3 2IGR 3, 370; vgl. K. LATTE, in: Gnomon 7, 1931, 117; L. MORETTI, IAG, Nr. 76; vgl. C. A. FORBES, in: CPh 50, 1955, $238 \mathrm{ff}$.

43 Diod. 12,82. Vgl. L. MORETTI, Olympionikai, Nr. 346; L. ROBERT, in: REG 741961149 n. 221; DERS., Rev. Phil. 41, 1967, $17 \mathrm{f}$.

${ }^{44}$ Vgl. L. MORETTI, IAG, Nr. 76; L. ROBERT, in: AE 1966, 110; vgl. J. JÜTHNER, $A L$ 2, 89, n. 182.

${ }^{45}$ Vgl. L. ROBERT, in: Hellenica 7, 1949, 111; DERS., in: AE 1966, 110; L. MORETTI, IAG, Nr. 76; vgl. auch J. JÜTHNER, $A L$ 2, 89 u. 92 n. 182; M. I. FI NLEY/H W PLEKET, Spiele, 116. ${ }^{46}$ Vgl. L. ROBERT, in: Hellenica 7, 1949, 111; DERS., Étud. anat., $138 \mathrm{ff}$.

47 Pind. Ol. 13,37; vgl.L. MORETTI, IAG, Nr. 76, 78; L. ROBERT, in: RA 1934, 1,52 ff.;

R. KNAB, Periodoniken, 5 f.; H. A. HARRIS, GAA, 75; J. EBERT, Epigramme, 123;

R. PATRUCCO, Sport, 95.

${ }^{48}$ Paus. 6,11,5;vgl. J. EBERT, Epigramme, Nr. 37.

49 IG 7,530; vgl. L. MORETTI, IAG, Nr. 26; DERS., Olympionikai, Nr. 850 ff.; vgl. auch DERS., $I A G$, Nr. 38, wo ein erfolgreicher Läufer auch im Heroldwettbewerb Sieger war. 
vielleicht sogar in zwei verschiedenen Alterklassen $^{50}$ - so wurde dies stolz vermerkt ${ }^{51}$. Besondere Wertschätzung genossen Läufer, die an einem Tage ${ }^{52}$ drei Laufkonkurrenzen für sich entscheiden konnten ${ }^{53}$. Solch erfolgreiche Athleten konnten sich mit dem Ehrentitel Dreifachsieger (трıaoтrís) schmücken ${ }^{54}$, was noch an Bedeutung gewann, wenn man diesen Erfolg in Olympia erzielte ${ }^{55}$. Diese Meisterleistung gelang Astylos von Kroton dreimal hintereinander (OI. Sp. 73-75 = 488-480 v. Chr. $)^{56}$ und dem wohl besten Läufer der Antike, Leonidas von Rhodos ${ }^{57}$ viermal (Ol. Sp. 154-157 $=164-$ 152 v. Chr. $)^{58}$. An dieser Stelle sei auch auf Polites aus Keramos in Karien verwiesen, der als einziger olympischer Triastes-Sieger (OI. Sp. $212=69$ n. Chr. $)^{59}$ neben den beiden Kurzstrecken statt des Waffenlaufes den

\footnotetext{
${ }^{50}$ Vgl. L. ROBERT, in: Hellenica 7, 1949, 112. Nach Paus. 6,2,10 f. hatte Damiskus von Messana mit 12 Jahren den Stadionlauf der Knaben in Olympia gewinnen können; vgl. L. MORETTI, Olympionikai, Nr. 417; H. BUHMANN, Sieg, 72.

${ }^{51}$ Vgl. L. MORETTI, IAG, Nr. 76, 78, 80.

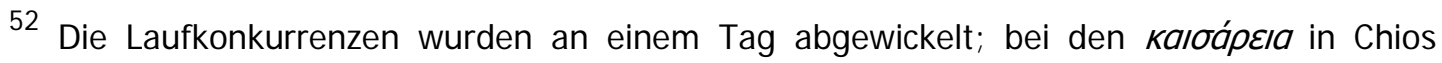
waren sie jedoch auf drei Tage verteilt; vgl. J. EBERT, Epigramme, 243.

${ }^{53}$ Vgl. L. MORETTI, IAG, Nr. 54, 57, 61.

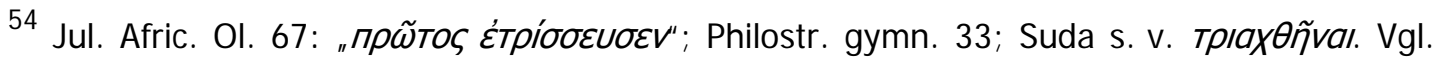
F. MEZÖ, Geschichte, 73; L. ROBERT, in: REG 71, 1958, 160; L. MORETTI, IAG, 155; J. EBERT, Pentathlon, 5; J. JÜTHNER, AL 2, 94; H. A. HARRIS, in: JHS 88, 1968, 138 f.; R. PATRUCCO, Sport, 124; J. SWADDLING, Games, 44; M. I. FINLEY/H. W. PLEKET, Spiele, 122; F. BREIN, GL 2, 105: „wenn wir von antiken Rekorden sprechen ... ist der Ehrentitel Triastes überliefert."

55 Der erste Triastes-Sieger in Olympia war Phanas von Pellene (OI. Sp. 67=512 v. ehr.). Vgl. L. MORETTI, Olympionikai, Nr. 142 ff.

${ }^{56}$ Vgl. L. MORETTI, Olympionikai, Nr. 178 f.; 186 f.; 196 ff.

${ }^{57}$ Paus. 6.13,4; vgl. F. MEZÖ, Geschichte, 73.

58 Jul. Afric. Ol. 157; Philostr. gymn. 33;vgl. L. MORETTI, Olympionikai, Nr. 618 ff; 622 ff; $626 \mathrm{ff} . ; 633 \mathrm{ff}$.

59 Paus. 6,13,3 f.; vgl. L. MORETTI, Olympionikai, Nr. 796 ff.
} 
Dolichos gewann und somit als der größte Allroundläufer der Antike gepriesen wurde ${ }^{60}$.

Einen anderen hochgeschätzten Ehrentitel konnte man in Plataiai beim bedeutendsten Waffenlauf erlangen. Dieser Wettkampf zeichnete sich dadurch aus, dass er über eine außergewöhnlich lange Distanz von etwa 15 Stadien (etwa 2.700 m) ging ${ }^{61}$, dass man bis in die Kaiserzeit im Gegensatz zu anderen Waffenläufen in voller Rüstung laufen musste ${ }^{62}$, aber auch höchste Preise ausgesetzt waren ${ }^{63}$. Der Sieger konnte sich mit der

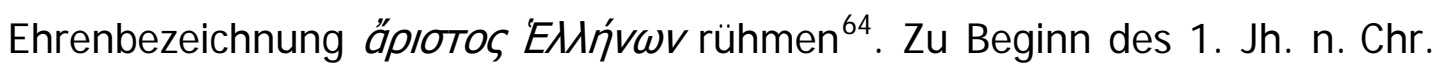
vermochte ein uns unbekannter Milesier diese Meisterleistung zu wiederholen ${ }^{65}$, was inm einen Sonderpreis einbrachte ${ }^{66}$. Siege in Folge bei

${ }^{60}$ Vgl. H. A. HARRIS, GAA, 75.

${ }^{61}$ Philostr. gymn. 8; Paus. 9,2,6;vgl. B. SCHRÖDER, Sport, 107, spricht fälschlicherweise von Mannschaftsrennen beim Waffenlauf im Zusammenhang mit den Darstellungen auf den Schilden.

62 Vgl. J. JÜTHNER, $A L$ 2, 122.

63 Paus. 9,2,6.

64 Vgl. L. ROBERT, in: REA 31, 1929, 13 ff.; DERS., in: RA 1934, 56 (hier auch der Hinweis, dass dieser Lauf von der Aristokratie bevorzugt wurde); DERS., Étud. anat., 141 f.; R. KNAB, Periodoniken, 14; L. MORETTI, IAG, 119; DERS., Olympionikai, Nr. 850 ff.; J. JÜTHNER, $A L$ 2, 124, n. 283; W.Decker,Sport,62.

65 Die von Philostr. gymn. 8 u, 24 aufgestellte Behauptung, dass ein Sieger in dieser Konkurrenz bei erneuter Teilnahme siegen müsse, anderweitig dem Tode geweiht sei (so noch E. N. GARDINER, GASF, 286 f.), ist zu verwerfen. Vgl. H. A. HARRIS, GAA, 75: ,...; this sounds like a story told by old hands in athletic dressing-rooms to gullible youngsters." Vgl. M. POLIAKOFF, Terminology, 146; W. E. SWEET, in: ZPE 50, 1983, 288.

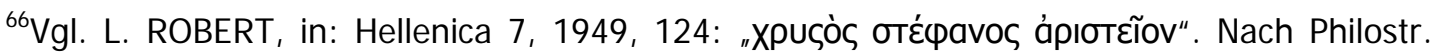
gymn. 24 schaffte es zu Beginn des 3. Jh. n. Chr. auch ein gewisser Optatus aus der Provinz Asia, den Waffenlauf zu Plataiai zweimal zu gewinnen. Vgl. R. MERKELBACH, in: ZPE 11, 1973, 128. 
bedeutenden Wettkämpfen ${ }^{67}$ wie bei Veranstaltungen, an denen man unmittelbar nacheinander teilgenommen hatte ${ }^{68}$, wurden zum einen als Zeugnis einer jahrelangen Spitzenstellung, zum anderen als Ausdruck einer absoluten Dominanz für einen bestimmten Zeitraum besonders hervorgehoben. Hierzu sind auch die Periodoniken zu zählen, die zahlreich unter den Läufern zu finden sind ${ }^{69}$. Einige von innen konnten die Periodos mehrfach gewinnen ${ }^{70}$; wobei Dandis von Argos $^{71}$, Mnasibulos aus Elatea ${ }^{72}$ und Philinos von $\mathrm{Kos}^{73}$ dieses Kunststück in verschiedenen Laufdisziplinen vollbrachten.

Siegeskataloge erfolgreicher Läufer können sich auf Erfolge in den panhellenischen Spielen und auch noch auf anderen bedeutenden Veranstaltungen beschränken, ${ }^{74}$ sie können aber auch sehr ausführlich mit detaillierten Zahlenangaben die Gesamtheit der Siege darstellen ${ }^{75}$. Nicht selten finden diese Listen ein Ende in einer konkreten Zahlenangabe über die

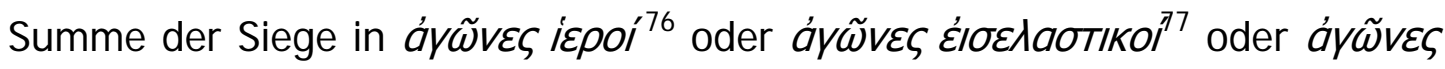

67 IvO 230; vgl. R. KNAB, Periodoniken, 39; E. BEY, in: BCH 28, 1904, 87 ff.; L. MORETTI, IAG, Nr. 24; 50; 78; DERS., Olympionikai, Nr. 294; 306; 312 (448-440 v. Chr.), Nr. 592; 595; 599; 605 (208-196 v. Chr.).

${ }^{68}$ Vgl. L. ROBERT, in: Hellenica 7, 1949, 118 ff.; M. N. TOD, in: CQ43, 1949, 109 n. 3.

${ }^{69}$ Vgl. R. KNAB, Periodoniken, $5 \mathrm{f}$.

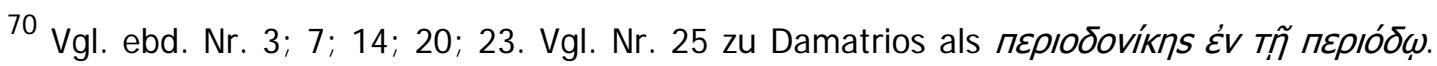
Vgl. auch St. DOW, in: Hesperia 4, 1935, 71 ff., wo ein Läufer sich als Periodonike rühmt, obwohl er im zerstörten Korinth nicht hatte starten können.

71 P. Oxy. 222; Jul. Afric. Ol. 76; Diod. 11, 53; Dionys. 9,37; vgl. R. KNAB, Periodoniken, Nr. 6; L. MORETTI, Olympionikai, Nr. 210; 222.

72 Paus. 10,34,5; IG 9,1,146; Syll.3 871; vgl. R. KNAB, Periodoniken, Nr. 50; L. MORETTI, Olympionikai, Nr. $868 \mathrm{f}$.

73 Paus. 6,17,2. Dieser Ausnahmeläufer kann zweifacher Periodonike sowohl im Stadion wie auch im Diaulos gewesen sein; vgl. L. MORETTI, Olympionikai, Nr. 550 ff. Vgl. auch Paus. 6,15,1, wo ein erst zwanzigjähriger Athlet schon Periodonike ist.

${ }^{74}$ Vgl. J. EBERT, Epigramme, Nr. 15; 20; 26; 35.

${ }^{75} \mathrm{Vgl}$. L. MORETTI, IAG, Nr. 59; 66; 76; 78.

${ }^{76}$ Vgl. L. ROBERT, Étud. anat., $138 \mathrm{ff}$. 


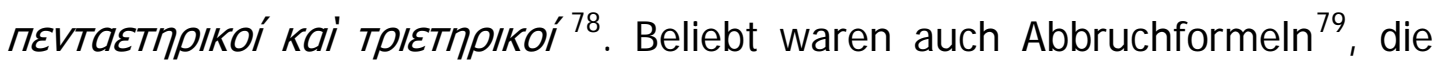
nach Auflistung einiger Siege zu erkennen geben, dass es schwer sei, die

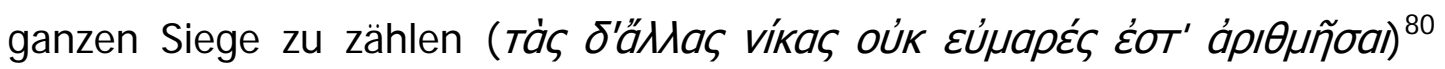

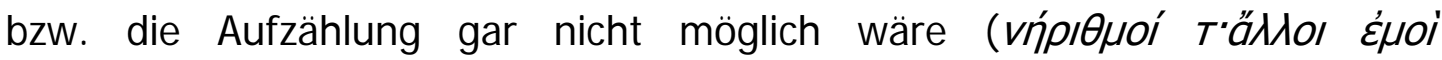
oré(pavol) ${ }^{81}$.

Die Erst- und/oder Einmaligkeit eines Triumphes wurde besonders gern und häufig hervorgehoben. Nur in Ausnahmefallen bezog sich dies auf einen neugeschaffenen Agon $^{82}$. Meist rühmte man sich in dieser Form bestimmter Siege oder Siegesserien ${ }^{83}$ - u. U. auf einen bestimmten Zeitraum begrenzt ${ }^{84}$ oder Siegeskombinationen ${ }^{85}$ in Bezug auf einen bestimmten Personenkreis ${ }^{86}$,

\footnotetext{
${ }^{77}$ Vgl. L.ROBERT, in: RA 1934, 57.
}

78 CIG 2682; vgl. L. MORETTI, IAG, Nr. 66; J. EBERT, Epigramme, Nr. 26; vgl. H. A. HARRIS, GAA, 75.

${ }^{79}$ Vgl. J EBERT, Epigramme, 68.

80 Anth. Pal. 13, 14; vgl. L. MORETTI, IAG, 64; J. EBERT, Epigramme, Nr. 15; ebd. Nr. 79, die Aufforderung, zu verharren und die Anzahl der Kämpfe zu studieren.

${ }^{81}$ Vgl. L. ROBERT, in: BCH 57, 1933, 539 ff.; in: SEG 19, 1956, 589; J. EBERT, in: WZ Halle 14, 1965, 5 ff.; DERS., Epigramme, Nr. 79.

82 CIG 2682; vgl. R. KNAB, Periodoniken, 40; L. MORETTI, IAG, Nr. 66.; DERS., Olympionikai,

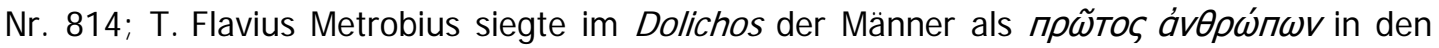
kapitolinischen Spielen zu Rom (86 n. Chr.). In der Inschrift CIL 6, 33950=I LS 5278 rühmt sich ein Läufer, der erste gewesen zu sein, der bei seinem ersten Start gleich erfolgreich war.

${ }^{83}$ Z. B. L. ROBERT, in: Hellenica 7, 1949, 117 ff., wo ein Athlet sich rühmt, als erster einen dreifachen Lauferfolg erzielt zu haben bei Wettkämpfen, die von dem ökumenischen Athletenverband ausgerichtet wurden. Vgl. C. A. FORBES, in: CPh 50, 1955, 240.

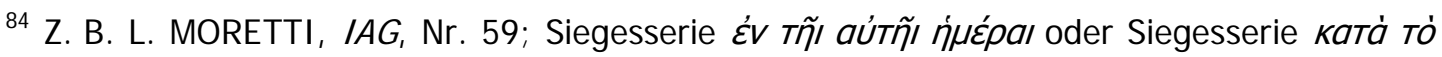

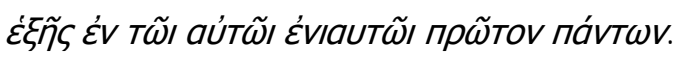

${ }^{85}$ Der Sieger des Stadionlaufes bei den pythischen Spielen (etwa Anf. d. 5. Jh. v Chr.), dessen Brüder im Faustkampf und Pankration bei derselben Veranstaltung erfolgreich waren,

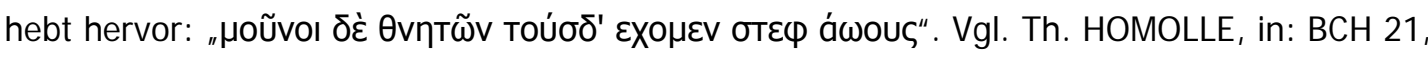
1897, 592 ff.; Syll 3 274; L. MORETTI, IAG, Nr. 29; J. EBERT, Epigramme, Nr. 43 ff. 
was viele Möglichkeiten offen ließ. Als herausragendes Beispiel sei hier die detaillierte Darstellung eines unbekannten Läufers aus Milet angeführt, bei dem die meisten Siege oder Siegesserien mit dem Vermerk der Erst- und Einmaligkeit versehen sind, entsprechend ihres Bezuges auf die Gesamtheit der Athleten, der Athleten Asiens, Ioniens oder seiner Heimatstadt ${ }^{87}$. Es liegt auf der Hand, dass die Periodoniken, die Triastes und die Träger des Titels

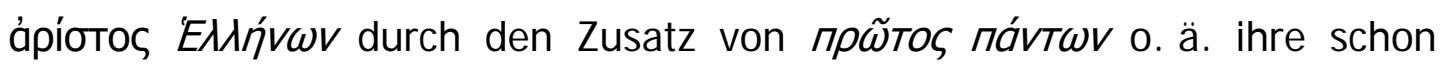
überragende Leistung noch zu steigern suchten ${ }^{88}$.

Bei den antiken Wettläufen kannte man mangels genauer Zeitmessinstrumente ${ }^{89}$ keine Zeitnahme ${ }^{90}$. Dennoch galt der Schnelligkeit bzw. Geschwindigkeit als auch der Dauerleistungsfähigkeit großes Interesse ${ }^{91}$. Nicht nur, dass erfolgreiche Läufer ehrenvolle Beinamen wie

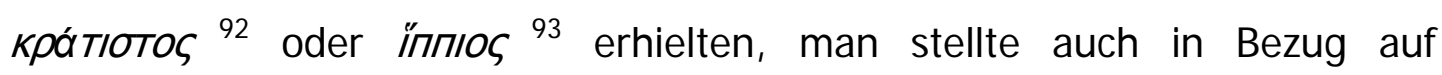
Schnelligkeit und Ausdauer Vergleiche zwischen Mensch und Tier an. Dem Polymnestor aus Milet wurde nachgesagt, dass er es mit den Hasen

${ }^{86}$ Auch in den nicht sehr zahlreichen Inschriften erfolgreicher Läuferinnen finden sich solche

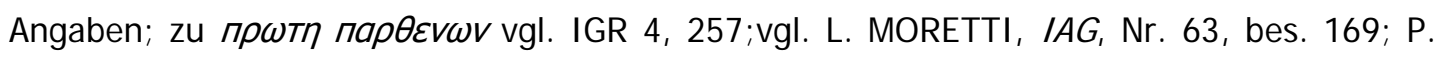
VEYNE, in: BCH 90, 1966, 146, n 4; L. ROBERT, in: REG 79, 1966, $731 \mathrm{ff}$.

${ }^{87}$ Vgl. L. ROBERT, in: Hellenica 7, 1949, 117 ff; L. MORETTI, IAG, Nr. 59.

88 Jul. Afric. Ol. 157; vgl. Anm. 87, wo u. a. die Erstmaligkeit des zweifachen Sieges im Waffenlauf zu Plataiai herausgestrichen wird. Vgl. R. KNAB, Periodoniken, 6; L. ROBERT, Lucillius, 183 n. 2 u. 192.

${ }^{89}$ Vgl. H. A. HARRIS, in: G \& R 7, 1960: „The Greek were mercifully free from the tyranny of the stop-watch."

${ }^{90}$ Vgl. E. N. GARDI NER, AAW, 140; F. MEZÖ, Geschichte, 71.

${ }^{91}$ In der griechisch-römischen Antike hatte man für das Zeitmaß einer Laufleistung Sinn und Interesse, was der Vergleich des schnellen Enthäutens eines Opfertieres mit der Zeit, die man für zwei Pferdedoppelläufe (Eur. El. 824 f.) brauchte, zeigt. Vgl. J. JÜTHNER, $A L$ 2, 92. 92 P Oxy., 222 zu Astylos aus Kroton. Vgl. F. MEZÖ, Geschichte, 73. Vgl. zu den Leistungen des Läufers L. MORETTI, Olympionikai, Nr. 178 f.

93 Paus. 6,13,3 zu Hermogenes aus Xantos; vgl. H. A. HARRIS, GAA, 65. Zu den Leistungen des Läufers vgl. L. MORETTI, Olympionikai, Nr. 805 ff. 
aufnehmen könne ${ }^{94}$, und dem Lasthenes von Theben wird ein Wettlauf zwischen inm und einem Rennpferd von Koroneia nach seiner Heimatstadt zugeschrieben ${ }^{95}$. Es war die Schnelligkeit, die bestimmten Läufern hoch angerechnet wurde und zu schillernden Vergleichen führte. Leonidas von Rhodos, der seine sensationelle Schnelligkeit über zwölf Jahre unter Beweis gestellt hatte, ${ }^{96}$ wurde als größter Läufer der Antike angesehen ${ }^{97}$. Der Ausnahmeläufer Ladas von $\operatorname{Argos}^{98}$ erweckte den Eindruck, dass er die Strecke durchfliege ${ }^{99}$ und keine Fußspur hinterlasse ${ }^{100}$.

Scherzhafterweise wurde die Laufzeit nahezu auf Null reduziert, da man bei der Schnelligkeit des Läufers den Eindruck hatte, dass Start und Ankunft am Ziel nahezu gleichzeitig vor sich gingen ${ }^{101}$.

Beim Wettlauf in den Stadien drückte sich die Überlegenheit des Läufers in seinem Abstand zu den Konkurrenten aus ${ }^{102}$. Dazu wurde der Abstand des Siegers zu seinen Verfolgern durch die Wettkampfrichter beim Überqueren der Zillinie durch den Sieger genau festgehalten ${ }^{103}$. Der in sportlichen

\footnotetext{
94 Jul. Afric. Ol. 46; Philostr. gymn. 13.

95 Diod. 14,11; vgl. L. MORETTI, Olympionikai, Nr. 352; J. JÜTHNER, AL 2, 107, n. 226.

96 Vgl. L. MORETTI, Olympionikai, Nr. 618 ff.; 622 ff; 626 ff; 633 ff.

97 Dio Chrys. orat. 31,126; Paus. 6,13,4. Vgl. R. KNAB, Periodoniken, 6; F. MEZÖ,
} Geschichte, 150; N. YALOURIS, Games, 265 ff; J. SWADDLING, Games, 44. Zu äрıбтоৎ

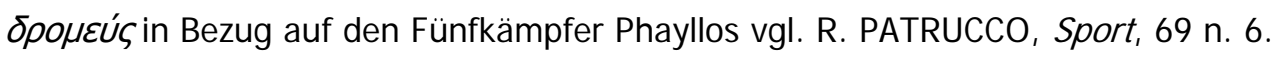

98 Vgl. L. MORETTI, Olympionikai, Nr. 260; J. EBERT, Epigramme, 180.

99 Anth. Pal. 4, 53 f.; vgl. W. W. HYDE, Monuments, 196 f.

100 Solin. col. rer. memor. 1,96; vgl. Paus. 2,19,7; Ov. met. 10,652; Stat. Theb. 6.

${ }^{101}$ Anth. Pal. 9,557; Lukian. Tim. 28.

102 So kann bei bildlichen Darstellungen (v. a. Vasenbildern), bei denen der führende oder gerade siegende Athlet mit beträchtlichem Abstand zu den folgenden Läufern dargestellt ist, seine Überlegenheit bzw. besondere Schnelligkeit zum Ausdruck gebracht werden. Vgl. J. JÜTHNER, $A L$ 2, 28; vgl. auch W. RUDOLPH, Kampfsport, 46.

103 Vgl. über die Ermittlung einer genauen Plazierungsskala bei einer größeren Läuferzahl durch das klug organisierte Kampfgericht J. EBERT, Pentathlon , 22. 
Dingen sachkundige Heliodor ist unser Gewährsmann dafür. Sein Held Theagenes gewinnt den pythischen Waffenlauf mit einigen Klaftern

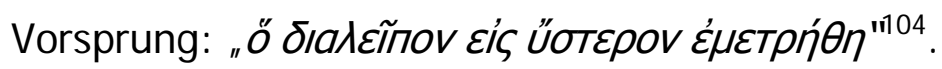

Zwar fehlen uns genaue Zeitangaben für den Bereich der klassischen Laufkonkurrenzen, doch haben wir eine Anzahl von Angaben über Laufleistungen bzw. zurückgelegte Distanzen mit zusätzlichem Vermerk der dafür benötigten Zeit ${ }^{105}$. Plinius d. Ä. ${ }^{106}$ berichtet von erstaunlichen Langlaufleistungen im Circus $^{107}$, bei denen ein Knabe von Mittag bis Abend eine Distanz von $L X X V$ passuum (etwa $111 \mathrm{~km}$ ) bewältigte ${ }^{108}$, ein Erwachsener, der den ganzen Tag gelaufen war, CLX passuum (etwa $237 \mathrm{~km}$ ) zurückgelegt hatte ${ }^{109}$. Ageos von Argos gewann den Dolichos bei den 133. Olympischen Spielen (328 v. Chr.) und meldete noch am selben Tage seinen Erfolg in dem über $100 \mathrm{~km}$ entfernten Heimatort ${ }^{110}$. Der Argiver

104 Aith. 4,4,1. Vgl. C. DIEM, Ein antiker Sportroman. Heliodors „Aethiopika”, Olympische Rundschau, 1941, H. 13,6.

105 Zum antiken Marathonlauf vgl. Plut. glor. Athen. 3,347; Lukian. laps. 3. Vgl. auch B. BILINSKI, L'antica oplite corridore di Maratona, Rom 1959, 1 ff.; J. SUOLAHTI, The Origin of the Story about the First Marathon-Runner, in: Arctos 5, 1957,127 ff.

106 Nat. 7,84; dazu F. J. FROST, in: AJ AH 4, 1979, 159: „Later writers ... more interested in absolute distance."

107 Vermutlich lief man wie die Pferde beim Wagenrennen um die Spina herum. Der Circus als Austragungsstätte zeigt eindeutig hier den rein sportlichen Charakter dieser UltraLangläufe.

108 Wenn man a meridie ad vesperum mit neun Stunden veranschlagt, so ist diese Distanz von einem Erwachsenen durchaus zu bewältigen, nicht aber wie Plinius uns mitteilt, von einem achtjährigen Knaben. Vgl. V. J. MATTHEWS, in: CW 68, 1974, 168; J. SUOLAHTI, in: Arctos 5, 1967, 131.

${ }^{109}$ Vgl. V. J. MATTHEWS, in: CW 68, 1974, 167 f., vertritt die Ansicht, dass diese Leistung nur möglich ist, wenn das tolerare in der Richtung zu interpretieren ist, dass diesem Rennen kein Zeitlimit zugrunde lag.

110 Jul. Afric. Ol. 113. Vgl. F. MEZÖ, Geschichte, 77; L. MORETTI, Olympionikai, Nr. 464;

W. DECKER, Sport, 61. 
Drymos, vielleicht um die Leistung seines Landsmannes zu überbieten ${ }^{111}$, brachte als Eilbote die Kunde von der Eröffnung der Spiele am selben Tage von Olympia in das über $140 \mathrm{~km}$ entfernt gelegene Epidauros ${ }^{112}$.

Rekordverdächtige Langlaufleistungen werden uns auch aus dem Bereich des Nachrichtenwesens gemeldet ${ }^{113}$, was den rein sportlichen Wert dieser

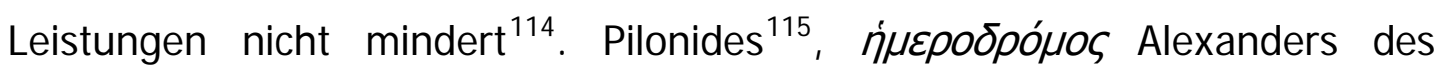
Großen, lief die Strecke von Sikyon nach Elis $(150-160 \mathrm{~km})^{116}$ nach unterschiedlichen Angaben in 9 Stunden ${ }^{117}$ bzw. an einem Tag $^{118}$. Herodot

${ }^{111}$ Da die Nachricht aus etwa der gleichen Zeit stammt, ist eine solche Vermutung nicht abwegig, vgl. J. JÜTHNER, $A L$ 2,107.

112 IG 4, 1349. M. FRAENKEL (Hrsg. der Inschrift) zweifelt an der Möglichkeit einer solchen Leistung. Anders H. BENGTSON, in: SO 32, 1956, $38 \mathrm{f}$.

113 Vgl. J. JÜTHNER, in: RE 8,1 (1912), s. v. Hemerodromos, 232 f.; W. RIEPL, Das Nachrichtenwesen des Altertums, Leipzig/Berlin 1913, 137 ff.; F. BREIN, GL 2, 104: „Von solchen Eilboten, den Hemerodromen („Tageläufern"), werden ganz unglaubliche Leistungen überliefert, an deren Richtigkeit aber nicht gezweifelt werden kann.“

114 Vgl. R. PATRUCCO, Sport, 41;W.Decker,Sport,61.

${ }^{115}$ Vgl. B. BILINSKI, in: Eos 50, 1, 1959-60, 72: „„... connu par son extraordinaire et presque fantastique record sportif."

116 Die von Plin. nat. 2,181 (mille et ducenta stadia) und 7,84 (MCCCV stadia) gegebenen Zahlen sind zu hoch gegriffen. Vgl. H. BENGTSON, in: SO 32, 1956, 38; V. J. MATTHEWS, in: CW 68, 1974, 165 f.; B. BILINSKI, in: Eos 50.1, 1959-1960, 76, hält an den Entfernungsangaben des Plinius fest, da Philonides einen Umweg über viele andere Orte gemacht habe.

117 Plin. nat. 2,181. H. BENGTSON, in: SO 32, 1956, 38 f., scheint eine solche Leistung in dieser knappen Zeit für unmöglich zu halten, hält jedoch den Rückweg, der mit 15 Stunden veranschlagt ist, für nicht ganz unglaubhaft (wohlgemerkt bei einer Distanz von 150160 km). Anders B. BI LINSKI, in: Eos 50, 1, 1959-60, 79: „... malgré leurs différences ils nous confirment un extraordinaire record de distance et de temps. "Ebd., 90, führt B. BILINSKI für die Möglichkeit solcher Ausnahmeleistungen die gesündere Lebensweise (!) der Alten an. 
berichtet, dass Pheidippides nach der Landung der Perser bei Marathon in zwei Tagen von Athen nach Sparta (mehr als $240 \mathrm{~km}$ ) gelaufen ist ${ }^{119}$, und von Euchiades wird gesagt, dass er rund $160 \mathrm{~km}$ (Plataiai - Delphi - Plataiai) an einem Tag bewältigt hat! ${ }^{120}$

Der antike Lauf ist in all seinen recht unterschiedlichen Formen geprägt von dem Bestreben einer möglichst präzisen Leistungsmessung. Neben der Schnelligkeit beeindruckte besonders die Bewältigung enormer Distanzen, wobei man auch die Laufstrecke in Relation zu der benötigten Zeit brachte.

118 Plin. nat. 7,84. V. J. MATTHEWS, in: CW 68, 1974, 166, hält an den Entfernungsangaben des Plinius fest und glaubt, dass auch eine solche Laufleistung erreichbar ist, wenn man uno die mit 24 Stunden gleichsetzt.

119 Hdt. 6,105 f.; Isokr. Panath. 24 spricht von einer Strecke von 1200 Stadien, während Plin. nat. 7,84 die Entfernung mit 1160 Stadien angibt.

120 Plut. Arist. 20 (1.000 Stadien). Vgl. B. BILINSKI, in: Eos 50.1, 1959-60, 78; V. J. MATTHEWS, in: CW 68, 1974, $162 \mathrm{f}$. 


\subsubsection{Pentathlon}

Das Pentathlon ${ }^{1}$ setzte sich zusammen aus den Disziplinen Sprung, Diskuswurf, Speerwurf, Lauf und Ringkampf ${ }^{2}$. Die drei erstgenannten Disziplinen wurden fast ausschließlich im Rahmen des Fünfkampfes betrieben

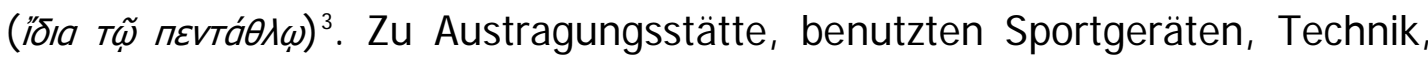
Regelwerk und Messung der Leistung sei hier auf die einzelnen Abschnitte dieser Arbeit verwiesen. Für den Stadionlauf ${ }^{4}$ und den Ringkampf ${ }^{5}$ galten die gleichen Bedingungen und Bestimmungen wie in der Spezialdisziplin. Wie in anderen Disziplinen kannte man auch im Pentathlon die Einteilung in Altersklassen ${ }^{6}$. Das Aufgebot an Kampfrichtern war ausgesprochen hoch, ${ }^{7}$

${ }^{1}$ Der Fünfkampf war bei den Alten trotz manchen Lobes (Arist. rhet. 1,5, 1361b; Ps.-Plat. amat. 135e) nicht sehr beliebt. So erfahren wir z. B. aus der Inschrift CIG 2758, dass der Preis für den Pentathloniken nur ein Viertel von dem der Kampfsportsieger betrug. Vgl. H. A. HARRIS, SGR, 33 („Cinderella of athletics"); I. WEILER, Sport, 190; H. SCHÖBEL, Olympia, 72; J. M. HEMELRIJ K, in: Hermeneus 37, 1966, 117, und V. OLIVOVÁ, Sport, 125 f, vertreten noch die längst überholte Meinung einer besonderen Wertschätzung des Fünfkampfes.

2 Die richtigen Disziplinen hatte bereits E. PINDER, Über den Fünfkampf der Hellenen, Berlin 1867, 177 ff., herausgearbeitet. Zweifel noch bei J. JÜTHNER, in: RE 19,1 (1937), s. v. Pentathlon, 525: „Vielleicht ist in römischer Zeit mit zunehmender Entartung des Sportes tatsächlich eine rohere Übung bevorzugt worden."

${ }^{3}$ Poll. 3, 151.

${ }^{4} \mathrm{~J}$. EBERT, Pentathlon, 12, denkt hierbei fälschlicherweise an einen 5-Stadien-Lauf.

${ }^{5}$ Vgl. W. RUDOLPH, Kampfsport, 29, wo er aber gleichzeitig auf die Unterschiede in den körperlich-konstitutionellen Voraussetzungen und die körperbildenden Werte hinweist.

${ }^{6}$ Vgl. L. MORETTI, IAG, 156 ff. Warum in Olympia das Knabenpentathlon nur einmal (38. Ol. Sp. $=628$ v. Chr.) ausgetragen wurde, ist umstritten. Paus. 5,9,1 sagt, daß der Gastgeber das Knabenpentathlon wieder absetzte, da ein Spartaner als erster siegte. H. A. HARRIS, SGR, 34, macht für die sofortige Abschaffung das geringe Zuschauerinteresse verantwortlich und J.SWADDLING, Games, 49, glaubt, dass die zu große körperliche Anstrengung den Ausschlag dafür gab. Vgl. auch S. G. MILLER, in: CSCA 8, 1975, 199 ff., der sich gegen ein Knabenpentathlon bei den Nemeen ausspricht. F. BREIN, GL 2, 119, meint, dass das Pentathlon überwiegend nur von Männern durchgeführt wurde.

${ }^{7}$ Paus. 5,9,5; vgl. Ph. E. LEGRAND, in: DS 4,1 (1907), s. v. Quinquertium, 804; L. MORETTI, in: RFIC 34, 1956, 57. 
und von einer Auslosung oder einem Ephedros bzw. Anephedros hören wir nichts ${ }^{8}$, doch wird, wenn nötig, beim abschließenden Ringkampf das Los die Paarungen bestimmt haben?.

Die Pentathloniken rühmten sich ihrer Leistungen und Erfolge weitestgehend in der gleichen Weise, wie es uns von den Schwerathleten und den Läufern her bekannt ist. So feiert das Pentathlon auch ákovití Siege ${ }^{10}$, wobei nicht klar ist, ob damit gemeint ist, dass der Gesamtsieg von vornherein zugestanden wurde ${ }^{11}$ oder nach Absolvierung einzelner Disziplinen ${ }^{12}$. Eindeutig dagegen ist die Art des Sieges, die ein Pentathlet aus Ephesos ćv

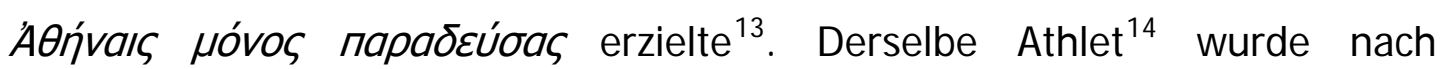

${ }^{8}$ Vgl. E. N. GARDI NER, GASF, 370. F. BREIN, GL 2,120.

'Vgl. J. EBERT, Pentathlon, 10 n. 3; R. MERKELBACH, in: ZPE 11, 1973, 264 n. 8; Wahrscheinlich wurden auch die Startpositionen beim Lauf ausgelost. Die Abfolge der Disziplinen war zumindest bei den bedeutenden Wettkämpfen festgeschrieben. Die Startfolge der Athleten in den einzelnen technischen Disziplinen wurde nach Ausweis einer rhodischen Inschrift (G. PUGLIESE-CARRATELLI, in: Annuario 30-32, 1952-1954, 289 f. Nr 65; L. MOREITI, in: RFIC 34, 1956; 55 ff.) durch die zuvor erbrachten Leistungen bestimmt. Vgl. R. PATRUCCO, Sport, 199 n.3; F. BREIN, GL 2, 119; M. I. FINLEY/H. W. PLEKET, Spiele, 117.

${ }^{10}$ Vgl. R. HAMPE/U. JANTZEN, in: J dl 52, 1937, 82 ff.; L. MORETTI, IAG, Nr. 8, J. JÜTHNER, $A L$ 2, 176 u. Abb. 52. Zu einem Unentschieden beim Pentathlon vgl. L. MORETTI, IAG, 243; G. E. BEAN, in: AJA 60, 1956, 367; J. EBERT, Pentathlon, 33; R. PATRUCCO, Sport, 209 f.; F. BREIN, GL 2, 120. Paus. 3,18,7 berichtet vom Tod des Fünfkampfsiegers Aenetus aus Sparta bei der Siegerehrung; vgl. C. A. FORBES, Accidents, $58 \mathrm{f}$.

${ }^{11}$ So J. EBERT, Pentathlon, 7; DERS., Epigramme, 53.

12 So L. MORETTI, IAG, 18 f.; R. PATRUCCO, Sport, 207; F. BREIN, GL 2, 120; DERS., in: Forschungen und Funde, Festschrift Berhard Neutsch, Hrsg. F. KRINZINGER: Innsbr. Beitr.

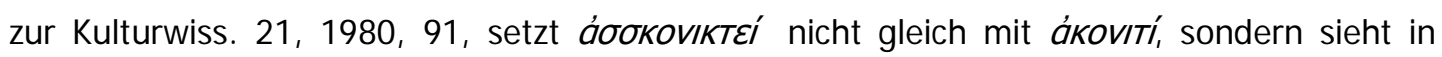
ersterem die Bezeichnung für einen vorzeitigen Gesamtsieg nach Erfolgen in drei Disziplinen vor dem abschließenden Ringen.

${ }^{13}$ Vgl. L. MORETTI, IAG, Nr. 75; J. EBERT, Epigramme, Nr. 9. Vgl. G. E. BEAN, in: AJA 60, 1956, 198. Anders H. A. HARRIS, in: JHS 82, 1962, 20 f.; vgl. auch L. ROBERT, in: RA 1978, $283 \mathrm{f}$. 
Aufzählung zahlreicher Pentathlonsiege gelobt, dass er im Diskuswurf

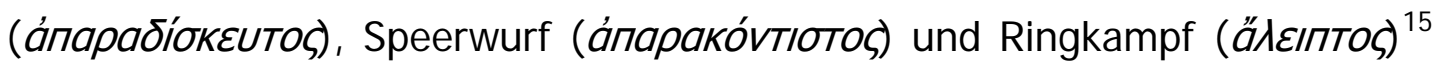
nicht besiegt worden war, was dann (nach Meinung vieler Gelehrter) zur Folge gehabt hätte, dass er alle Fünfkämpfe, an denen er teilgenommen hat, für sich entscheiden konnte $^{16}$. Ähnlich erfolgreich war ein junger spartanischer Pentathlet. Alle bedeutenden Fünfkämpfe, zu denen er antrat,

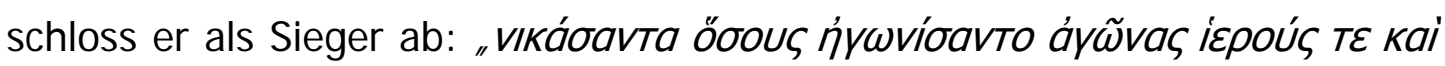

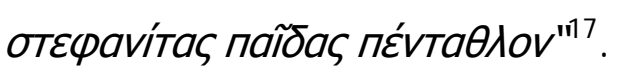

Ein anderes Mittel zur Hervorhebung besonderer Leistung war die Angabe der Zahl der bezwungnen Gegner. So wird von einem Athleten berichtet, der über 87 Konkurrenten triumphierte ${ }^{18}$. Aber auch die Zahl der Gegner im

${ }^{14}$ Vgl. L. MORETTI, IAG, Nr. 75; J. EBERT, Epigramme, Nr. 9. Vgl. auch R. KNAB, Periodoniken, 15.

15 J. JÜTHNER, $A L$ 2, 347 n. 129, übergeht diese Möglichkeit ganz. Vgl. J. EBERT, Pentathlon, 5, wo weitere Fünfkampfsieger aufgeführt werden, die im Speerwurf, Diskuswurf und Ringkampf erfolgreich waren.

${ }^{16}$ Selbst ein Vertreter des Punktesystems wie L. MORETTI, IAG, 17 f., plädiert für einen Gesamtsieg nach Erfolgen in drei Konkurrenzen, und bei einem reinen Punktesystem hätte dieser Athlet in den beiden restlichen Disziplinen äußerst schlecht abschneiden müssen, um nicht Gesamtsieger zu werden.

${ }^{17}$ IG 5,1,688. Vgl. L. ROBERT, Lucillius, $183 \mathrm{f}$.

${ }^{18}$ Vgl. M. GOUGH, Anat. Stud. 2, 1952, 127; L. MORETTI, IAG, Nr. 86 . G. E. BEAN, in: AJA 60,1956, 363 n. 9, weist daraufhin, dass dies nicht einmal ein Rekord ist, da nach TAM 2, 301 n. 304, Schwerathleten 8 bzw. 9 Runden durchzustehen hatten und somit eine insgesamt höhere Zahl an Kontrahenten hinter sich ließen (was bei 8 Runden nicht sicher zu entscheiden ist, d. V.). L. MORETTI, IAG, 255, sieht darin die Gesamtzahl der Gegner in den Ausscheidungsrunden. J. EBERT, Pentathlon, 23, hält eine solch hohe Teilnehmerzahl für nur einen Fünfkampf aus zeitlich-organisatorischen Gründen für unmöglich. R. PATRUCCO, Sport, 22, versteht darunter die Gesamtzahl der Athleten bei den Vorläufen eines Fünfkampfes. Vgl. auch L. ROBERT, Lucillius, $184 \mathrm{f}$. 
abschließenden Ringkampf konnte ein Spiegelbild der Überlegenheit des Siegers $\operatorname{sein}^{19}$.

Wie Sieger in anderen Disziplinen pflegten auch die Pentathloniken, soweit möglich, anderweitige Siege anzuführen. Nicht vielen von ihnen war ein Sieg in Laufkonkurrenzen vergönnt ${ }^{20}$, und noch seltener war innen ein Sieg in einem der schwerathletischen Spezialwettkämpfe gelungen ${ }^{21}$. Eine große Ausnahme $^{22}$ war es, wenn ein siegreicher Fünfkämpfer zusätzlich Siege sowohl in leicht- wie in schwerathletischen Spezialkonkurrenzen davontrug ${ }^{23}$. Natürlich waren auch zwei oder mehr Siege ${ }^{24}$ an einem Tag außergewöhnlich

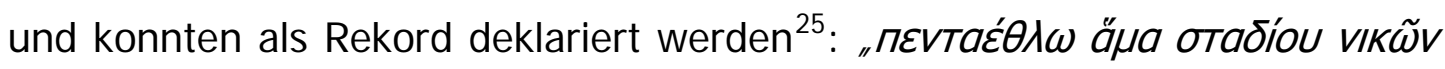

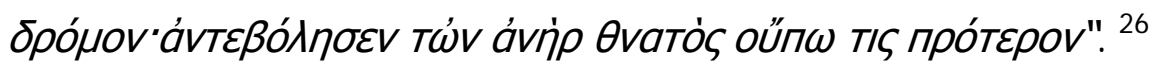

19 So hätte bei einem System, das nur Sieger in Einzelkonkurrenzen zum abschließenden Ringkampf zuließ, der spätere Gesamtsieger, der nur noch einen Ringkampf bestreiten musste, mit seinem letztlich unterlegenen Gegner die Konkurrenz beherrscht (jeder zwei Einzelsiege) und war durch seinen Ringsieg sogar ein (nicht vorzeitiger, dafür aber absoluter) Dreifachsieger. Vgl. aber Bakchyl. 9,38.

20 Pind. Ol. 13,31; J ul. Afric. Ol. 96; Paus. 2,11,8 (der Sikyonier Aelius Granianus, der neben dem Pentathlon in drei verschiedenen Laufkonkurrenzen erfolgreich war); 6,15,9; 10,9,2; vgl. L. MORETTI, IAG, Nr. 60; 61; 82; 86; DERS., Olympionikai, Nr. 249; 417; 848; 961; 922.

${ }^{21}$ IG 2/32, 2314; Paus. 5,9,1 u. 6,15,8; vgl. L. MORETTI, Olympionikai, Nr. 63 f.; J. EBERT, Epigramme, Nr. 1.

${ }^{22}$ Dies bezieht sich natürlich auf große und bedeutende Feste; bei kleineren lokalen Veranstaltungen wird es dagegen nicht selten gewesen sein, dass ein Pentathlonike auch in mehreren Spezialdisziplinen Erfolge davontrug.

23 Syll.3 1065; in: IGR 4, 1064; vgl. L. MORETTI, IAG, Nr. 60. M. N. TOD, in: CQ 53, 1949, 110 n. 4. Zu einem Sieger im Pentathlon und Apobatenwettkampf vgl. L. ROBERT, Annuaire de l'École des Hautes Etudes 4. Sect. 1964-1965, 180.

24 Syll.3 1066; IGR 4, 1065; vgl. L. MORETTI, IAG, Nr. 61.

${ }^{25}$ E. N. GARDI NER, in: J HS 23, 1903, 61 n. 34: „Xenophon's double victory in one day was a record."

${ }^{26}$ Pind. Ol. 13, $30 \mathrm{f}$. 


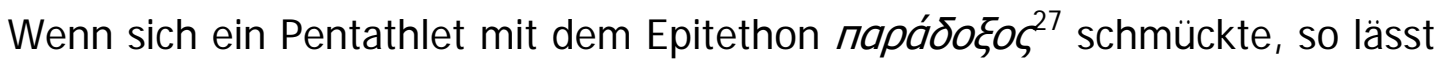
das nur auf einen zusätzlichen Sieg in einer Spezialdisziplin schließen, jedoch nicht auf zwei Pentathlonsiege an einem Tage. Auch Fünfkampfsiege in Folge, sei es bei verschiedenen Veranstaltungen ${ }^{28}$ oder bei ein und demselben Fest ${ }^{29}$, galten gerade bei Pentathleten als Ausnahme und wurden besonders herausgestellt. Natürlich pflegten auch einige erfolgreiche Fünfkämpfer ihre Siege detailliert in Siegeskatalogen aufzuführen ${ }^{30}$, andere wiederum maßen ihre Erfolge durch eine konkrete Zahlenangabe ihrer Siege

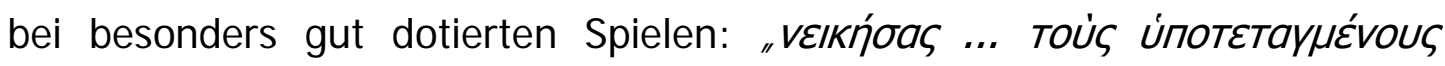

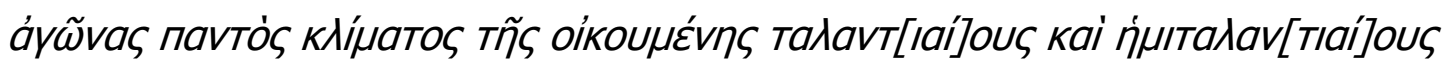
$\mu \zeta{ }^{\prime \prime 131}$

Doch gewinnt man den Eindruck, dass es für Fünfkämpfer nicht leicht war, zahlreiche Siege zu erlangen ${ }^{32}$. Selbst der berühmte Phayllos von Kroton konnte nur zwei Pentathlonsiege bei den Pythien und einen bei den Isthmischen Spielen vorweisen ${ }^{33}$. Der Eleer Gorgos hielt den Rekord in Olympia mit ganzen vier Siegen ${ }^{34}$.

27. MORETTI, IAG, Nr. 75.

28 Paus. 6,2,11; vgl. M. GOUGH, Anat. Stud. 2, 1952, 127 ff.; L. MORETTI, IAG, Nr. 86; in: SEG 12, 1955, 512.

${ }^{29}$ Vgl. L. MORETTI, IAG, Nr. 75.

30 Syll.3 1066; IGR 4, 1065; vgl. L. MORETTI, IAG, Nr. 61; 72.

${ }^{31}$ Vgl. M. GOUGH, Anat. Stud. 2, 1952, 127 ff.; L. MORETTI, IAG, Nr. 86.

32 So auch R. KNAB, Periodoniken, 7 u. 9. Der Versuch W. RUDOLPHs, Kampfsport, 55 f., das Gegenteil zu beweisen, scheitert völlig.

33 Paus. 10,9,2; vgl. W. DECKER, Sport, 86.

34 Paus. 6,15,9; vgl. L. MORETTI, Olympionikai, Nr. 961-966. Zu den außerordentlich erfolgreichen Pentathleten ist auch der Korinther Nikolades mit drei isthmischen, drei nemeischen und einem pythischen Sieg zu zählen, Anth. Pal. 13, 19. 
Periodoniken im Fünfkampf sind uns nicht überliefert ${ }^{35}$, wobei der Eleer Timon als einziger hätte Periodonike werden können, wäre inm nicht als Eleer die Teilnahme an den Isthmischen Spielen verwehrt gewesen ${ }^{36}$.

Auch Fünfkämpfer pflegten die Erstmaligkeit eines Sieges anzuzeigen ${ }^{37}$, und es ist bezeichnend, dass ein Spottgedicht des Lucillius diese Rekordmanie aufgreift und einen völlig unfähigen und in allen Disziplinen klar geschlagenen Pantathleten als Ersten in dieser Weise bezwungenen darstellt:

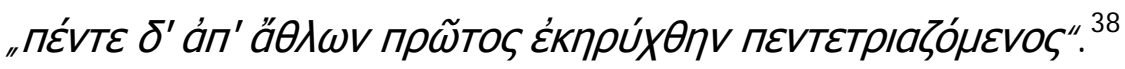

Abschließend sei noch auf die ständig aktuelle Diskussion betreffs der Reihenfolge der Disziplinen und des Systems der Siegerermittlung eingegangen. Da die Quellen keine eindeutige Festlegung der Reihenfolge zulassen - mit Ausnahme des Ringkampfes als letzter Disziplin ${ }^{39}$ - wurde fast jede denkbare Reihenfolge der Disziplinen ersonnen ${ }^{40}$. Noch einfallsreicher war man bei der Erstellung von Systemen zur Siegerermittlung ${ }^{41}$, wobei immer wieder völlig konträre Lösungsvorschläge gemacht wurden ${ }^{42}$. Das

${ }^{35}$ Vgl. R. KNAB, Periodoniken, 7.

36 Paus. 5,2,5; 6,16,2. Vgl. R. KNAB, Periodoniken, 9; L. MORETTI, Olympionikai Nr. 601; H. BUHMANN, Sieg, $77 \mathrm{f}$.

37 Syll. 3 1065; IGR 4, 1064; vgl. L. MORETTI, IAG, Nr. 60; J. EBERT, Epigramme, Nr. 71.

38 Anth. Pal. 11,84. Vgl. J. EBERT, Pentathlon, 15 f.; L. ROBERT, Lucillius, 237 ff., bes. 239 ก. 2.

39 Hdt. 9,33; Paus. 3,11,6; Xen. hell. 7,4,29; Bakchyl. 9,27 ff.; Artem. 1,57.

${ }^{40}$ Eine übersichtliche Darstellung der verschiedenen Lösungsmöglichkeiten bietet I. WEI LER, Sport, 191. E. D. FLOYD, in: TAPhA 96, 1965, 145, spricht von einer Umstellung der Reihenfolge der Wettkämpfe in späterer Zeit. E. N. GARDINER, in: JHS 23, 1903, 69; L. MORETTI, in: RFIC34, 1956, 60, und F. BREIN, GI 2, 120, sprechen von abweichenden Lösungen zu verschiedenen Zeiten an verschiedenen Orten.

${ }^{41}$ Eine Darstellung zahlreicher Modelle bei E. N. GARDINER, in: JHS 23, 1903, 54 ff.; J. EBERT, Pentathlon, $20 \mathrm{ff}$.

${ }^{42}$ Vgl. R. PATRUCCO, Sport, 217 ff.; W. E. SWEET, in: ZPE 50, 1983, 287 ff. 
Spektrum ${ }^{43}$ reicht von der ausschließlichen Notwendigkeit erster Plätze in den einzelnen Disziplinen zum Erlangen des Gesamtsieges ${ }^{44}$ über das „Prinzip des dreifachen relativen Sieges ${ }^{445}$ bis zu einer reinen Punktebewertung, ${ }^{46}$ und dies alles mit nuancenreichen Varianten ${ }^{47}$. Bei einer besonderen Wertigkeit der Siege in den einzelnen Disziplinen, was auch für das System des Prinzips der relativen Siege zutrifft, war ein Gesamtsieg mit drei Siegen

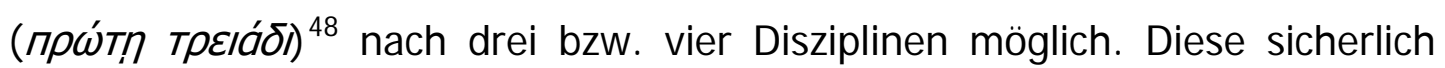
nicht häufig vorkommende Form des vorzeitigen Gesamtsieges ${ }^{49}$ brachte dem Sieger den Ehrentitel трıакти́p ein ${ }^{50}$. Eine Punktewertung, wie sie schon von verschiedenen Gelehrten erwogen wurde ${ }^{51}$, ist von Patrucco konsequent weitergedacht und ausgebaut worden ${ }^{52}$. Bei seinem Modell erhalten

43 Alle neueren Lösungsversuche, so gegensätzlich sie sein mögen, greifen nur seit Jahrzehnten bekannte Lösungsversuche neu auf und modifizieren sie. Dabei erschweren nicht nur die sich z. T. widersprechenden Quellen, sondern auch die Berücksichtigung sportpraktischer Überlegungen wie organisatorischer Bedingtheiten eine allseits befriedigende Lösung.

${ }^{44}$ Vgl. H. A. HARRIS, GAA, 77 ff.; W. E. SWEET, in: ZPE 50, 1983, $287 \mathrm{ff}$.

${ }^{45}$ Vgl. J. EBERT, Pentathlon, 30 ff.; DERS., in: ZPE 13, 1974, 257 ff.; DERS., Olympia, 59 ff.

${ }^{46}$ Vgl. R. PATRUCCO, Sport, $217 \mathrm{ff}$., wo auch ein Ausscheidungsverfahren und ein vorzeitiger Sieg durch Erfolge in drei Disziplinen abgelehnt wird. Allerdings beschränkt sich dieser Lösungsvorschlag auf die Kaiserzeit.

${ }^{47}$ Vgl. L. PIHKALA/E. N. GARDINER, in: JHS 45, 1925, 132 ff.; G. E. BEAN, in: AJA 60, 1956; H. A. HARRIS, in: G\&R 19, 1972, 60 ff.; R. MERKELBACH, in: ZPE 11, 1973, 261 ff. vgl. dazu die Gegenschrift von J. EBERT, in: ZPE 13, 1974, $257 \mathrm{ff}$.

${ }^{48}$ Vgl. H. W. BUCKLER, in: JHS 37, 1917, 88 ff., Nr. 1; IGR 4, 1761; L. MORETTI, IAG, Nr. 82; vgl. Schol. Aristid. Panath. 3,339d.

${ }^{49}$ Vgl. F. BREIN, GL 2, 120; W. E. SWEET, in: ZPE 50, 1983, 289; anders J. EBERT, Pentathlon, 6.

${ }^{50}$ Vgl. J. EBERT, Pentathlon, 6 u. 18; R. PATRUCCO, Sport, 211; F. BREIN, GL 2, 120; J. SWADDLING, Games, 49; I. WEI LER, Sport, 194.

${ }^{51}$ Vgl. L. MORETTI, IAG, 17 f.; Vgl. auch R. MERKELBACH, in: ZPE 11, 1973, 266.

${ }^{52}$ Vgl. R. PATRUCCO, Sport, 217 ff. Allerdings wies schon J. JÜTHNER, in: RE 19.1 (1937), s. v. Pentathlon, 527 f., darauf hin, dass es keine antiken Hinweise auf ein Punktesystem gibt. G. E. BEAN, in: AJA 60, 1956, 363, und J. EBERT, Pentathlon, 25 f., lehnen eine 
entsprechend der Teilnahmerzahl der Sieger n-Punkte, der Zweitplatzierte n1-Punkte, etc., ${ }^{53}$ was ein einfach durchzuführendes System darstellt, das dann über Zwischenergebnisse zu einem Endresultat führt ${ }^{54}$. Dabei ist eine präzise Siegerermittlung und eine genaue Wertschätzung des Sieges in Bezug auf die Teilnehmerzahl wie auch der Abstand der Konkurrenten untereinander gut darstellbar.

Es lässt sich festhalten, dass beim Pentathlon neben einem hohen organisatorischen Niveau eine ausgeprägte Form der Sportbürokratie vorzufinden ist. Zum Aspekt der Siegerermittlung ist nach wie vor die von J. EBERT vorgebrachte Lösung grundlegend und richtungsweisend.

Punktebewertung ab und sehen die Schwäche dieses Systems v. a. in der unbefriedigenden Art und Weise der Ringkampfbewertung.

${ }^{53}$ Vgl. R. PATRUCCO, Sport, 219.

54 R. PATRUCCO, Sport, 221: ,... al termine di ogni prova risultava una classifica parziale, da cui, alla fine della competizione, deriva una classifica generale finale; le classifiche di merito erano realizzate con un semplice sistema di punteggio relativo." 
Der Ballsport ${ }^{1}$, trotz seines Fehlens bei den bedeutenden Sportveranstaltungen ${ }^{2}$, war weit verbreitet und erfreute sich allgemein großer Beliebtheit ${ }^{3}$. Ballspielformen unterschiedlichsten Schwierigkeitsgrades eröffneten Spielmöglichkeiten für Jung ${ }^{4}$ und Alt $^{5}$ und auch das weibliche Geschlecht ${ }^{6}$, wobei neben dem gesundheitserhaltenden und -fördernden Aspekt ${ }^{7}$ der Wettkampf nicht zu kurz kam ${ }^{8}$.

Das Altertum kannte eine Vielzahl von verschiedenartigen Bällen ${ }^{9}$, die sich hinsichtlich der Größe, des Materials und der Füllung deutlich

${ }^{1}$ Zum Ballspiel mit Tanz vgl. Hom. Od. 6,100 ff.; 8,370 ff; Athen. 1,15c. Vgl. H. HOMMEL, Tanzen und Spielen, in: Gymnasium 56, 1949, 201 ff.; S. MENDNER, Das Ballspiel im Leben der Völker, Münster 1956, 86 ff.

${ }^{2}$ Vgl. H. A. HARRIS, GAA, 24; F. BREI N, GL 2, 161.

${ }^{3}$ Z. B. Sen. de brev. vit. 13, 1; vgl. L. GRÜNDEL, Griechische Ballspiele, in: AA 40, 1925, 89; E. WEGNER, Das Ballspiel der Römer, Diss., Würzburg 1938, 5 f.; C. DIEM, Weltgeschichte, 293; W. BACKHAUS, GL 2, 233.

4 Vgl. M. GOEPEL, Beiträge zur Geschichte des Ballspiels, Progr. Eberswalde 1909, 4; E. N. GARDINER, $A A W, 235$.

5 Mart. 14, 47; Suet. Aug. 83; Plin. epist. 3, 1, 8 hält das Ballspiel für ein geeignetes Mittel gegen das Altern.

${ }^{6}$ ggl. L. BERNABÒ BREA, Musei e monumenti in Sicilia, Novara 1958, 144; A. METZNER, Leichtathletik im Bikini. Ein beschaulicher Besuch bei den Sportmosaiken der Kaiservilla in Piazza Armerina, in: Leichtatheltik 11, 1960, 1204 ff.; M. DOLCH, Wettkampf, Wasserrevue oder diätische Übungen? Das Mosaik mit den zehn Mädchen in der römischen Villa bei Piazza Armerina auf Sizilien, in: Nikephoros 5, 1992, 173-189; G. LUKAS, Rom, Abb. 64; H. M. LEE, Athletics and the Bikini Girls from Piazza Armerina, in: Stadion 10, 1984, Fig. 1.

${ }^{7}$ Bes. Galen, de parv. pil. 1; vgl. E. WEGNER, Ballspiel, 40 f.; S. MENDNER, Ballspiel, $122 \mathrm{ff}$.

${ }^{8}$ Vgl. R. PATRUCCO, Sport, 335; I. WEILER, Sport, 210 ff.; Anders F. EPPENSTEINER, Der Sport, München 1964, 22; H. A. HARRIS, SGR, 80.

9 Mart. 7, 32, 7 ff.; 12, 82, 5; 14, 45; Anth. Pal. 12, 44; 14, 62; Poll. 9, 104 ff.; vgl E. WEGNER, Ballspiel, 9 ff.; S. MENDNER, Ballspiel, 77 ff.; W. RUDOLPH, in: Klio 48, 1967, 87 f.; H. A. HARRIS, SGR, 78 ff; W. BACKHAUS, GL 2, 233 f., M. DOLCH, in: Stadion 7, 1981, 53 ff. 
unterschieden ${ }^{10}$. Die sich daraus ergebenden unterschiedlichen Spieleigenschaften der Bälle hatten die Verwendung bestimmter Bälle für bestimmte Spielformen zur Folge ${ }^{11}$. Als Austragungsort für Ballspiele konnten freie Plätze oder Straßen dienen ${ }^{12}$, man verfügte aber auch über speziell dem Ballsport zugedachte Räumlichkeiten (Sphaeristerium) ${ }^{13}$, die oft dem Gymnasium, der Palästra oder den Thermen angeschlossen waren oder auch die Villen der Reichen zierten ${ }^{14}$. Ballspieler konnten in Clubs ${ }^{15}$ zusammengeschlossen sein, und wir wissen auch von speziellen Ballspieltrainern ${ }^{16}$ und professionellen Ballspielern ${ }^{17}$.

Die Zahl der Spielformen ${ }^{18}$ war beträchtlich und reichte von Einzelübungen über Spiele in Kleingruppen ${ }^{19}$ bis zu großen Mannschaftsspielen ${ }^{20}$. Bei

10 Zu Glasbällen (vitreae pilae) vgl. ClL 6, 9797=ILS 29; H. GEIST, Römische Grabinschriften, München 1969, 146; zu Kegelkugeln vgl. A. MAU, Pompeji in Leben und Kunst, Leipzig 1908², 94; S. MENDNER, Ballspiel, 79, 114, 116 u. Taf. 12a.

${ }^{11}$ Vgl. E. N. GARDINER, AAW, 232; R. PATRUCCO, Sport, 348.

12 Sen. epist. 104, 33; Hor. sat. 1, 6, 126; 2, 6, 48; Strab. 5, 3, 8.

13 Vgl. L. SCHNEIDER, in: RE 2, 3 (1927), s. v. Sphairisterion, 1680 ff.; S. MENDNER, Ballspiel, $79 \mathrm{ff}$.

${ }^{14}$ Theophr. char. 5, 9; Plin. epist. 2, 17, 12; 5,6,27; Suet. Vesp. 20; Dig. 17, 1, 16.

${ }^{15}$ CIL 4, 1147; vgl. G. LAFAYE, in: DS 4 (1890), s. v. pilarius, 145 f.; L. FRIEDLÄNDER, Cena Trimalchionis, Leipzig 1906, 50.

16 Athen. 1, 15 a; Galen, de. parv. pil. 4. Vgl. E. N. GARDINER, AAW, 229; E. WEGNER, Ballspiel, 8; R. PATRUCCO, Sport, 335.

17 ClL 6,8997; 6, 9797;12,4501; Eustath. 1601,46; Anth. Pal. 5,214.Vgl. E. N. GARDINER, AAW, 229; S. MENDNER, Ballspiel, 79; H. A. HARRIS, SGR, 105; R. PATRUCCO, Sport, 36; I. WEI LER, Sport, 21.

18 S. MENDNER, Ballspiel, 81 f., gliedert die antiken Ballspiele in drei Kategorien:

a) gebenderweise spielen (datatim /udere),

b) schlagenderweise spielen (explusim ludere);

c) raffenderweise spielen (raptim /udere). 
Schlagspielen wie Aporrhaxis ${ }^{21}$ und Anakrusia ${ }^{22}$, bei denen der Ball gegen den Boden ${ }^{23}$ bzw. gegen eine Wand ${ }^{24}$ geprellt wurde, zählte man selber oder ließ von den Mitspielern die Ballkontakte zählen ${ }^{25}$. Durch Vergleich der Ergebnisse wurde der Sieger ermittelt.

Ähnlich verfuhr man bei einem Spiel, bei dem es galt, den Ball in der Luft zu halten $^{26}$, wobei die zu Boden gefallenen Bälle gezählt wurden ${ }^{27}$. Als Hilfspersonal reichten Diener neue Bälle und vermerkten das Ergebnis. Eine attische Grabflasche (4. Jh. v. Chr.) ${ }^{28}$ zeigt einen jungen Mann, dessen Hände auf dem Rücken verschränkt sind und der einen Ball in der Größe eines Fußballes ${ }^{29}$ auf dem Oberschenkel springen lässt ${ }^{30}$. Der Knabe neben

19 Zum Ephedrismos, ein Ballspiel, bei dem ein Fangfehler den Tausch der Positionen von Reiter und Pferd bewirkte, vgl. P. ZAZOFF, Ephedrismos. Ein altgriechisches Spiel, in: A \& A 11, 1962, $35 \mathrm{ff}$.

${ }^{20}$ Ob unter dem Begriff paganica ein Ballspiel zu verstehen ist, bei dem das ganze Dorf auf dem Lande (pagus) beteiligt war, bleibt umstritten, vgl. J. MARQUARDT, Privatleben, 842; H. BLÜMER, Privataltertümer, 441; E. WEGNER, Ballspiel, 34 ff.; S. MENDNER, Ballspiel, 79.

${ }^{21}$ Poll. 9, 105; vgl. auch Sen. nat. 10; Stat. silv. 1,5,57 ff.

${ }^{22}$ Schol. Plat. Theait. 146a; Poll. 9,105; Hesych. s. v. ávaкров sía.

23 Vgl. G. SCHNEIDER-HERRMANN, Der Ball bei den Westgriechen, in: BABesch 46, 1971, Abb. 3 u. 4.

${ }^{24}$ Vgl. G. LAFAYE, in: DS 4,1 (1907), s. v. Pila, Fig. 5666; S. MENDNER, Ballspiel, Taf. 15 d.

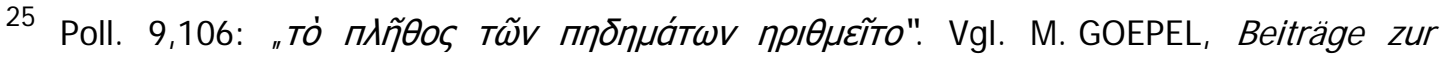
Geschichte des Ballspiels, Eberswalde 1909, 13; G. SCHNEIDER-HERRMANN, in: BABesch 46, $1971,125$.

${ }^{26}$ Ausführlich dazu E. WEGNER, Ballspiel, 24 ff.

27 Petron. 27: „alter numerabat pilas, non quidem eas, quae inter manus lusu expellente vibrabant, sed eas, quae in terram decidebant".

${ }^{28}$ Vgl. E. N. GARDINER, AAW, Fig. 210; S. MENDNER, Ballspiel, Taf. 12b; C. DIEM, Weltgeschichte, Abb. 122; H. A. HARRIS, SGR, Fig. 4; N. YALOURIS, Games, Fig. 149; R. PATRUCCO, Sport, Fig. 166.

29 Einige Gelehrte sehen darin einen weiteren Beweis für die Existenz einer Form des Fußsballspiels in der Antike (z. B. Isid. 18, 69, 1: suram dicitur dare, qui eure prolato 
inm scheint die Zahl der Ballkontakte festzuhalten. Bei Einzel- wie Mannschaftsspielen scheint man - sei es der Spieler selbst oder dafür eingeteilte Personen - die Ballkontakte gezählt bzw. das Spielergebnis ständig laut bekannt gegeben $\mathrm{zu}$ haben ${ }^{31}$, was auch einige als störend empfinden konnten: "si vero pilicrepus supervenit et numerare coepit pilas, actum est ${ }^{\text {‘22 }}$. Das Spiel mit mehreren Bällen spielte v. a. auf eine Höchstzahl von gleichzeitig im Fluss gehaltenen Bällen $a b^{33}$. Einen wahren Meister in dieser Disziplin zeigt uns eine römische Grabsäule ${ }^{34}$ : Der dargestellte Spieler hält mit Händen und Füßen sieben Bälle gleichzeitig in der Luft ${ }^{35}$.

Beim Trigon $^{36}$, bei dem sich drei Spieler ${ }^{37}$ im Dreieck aufgestellt ${ }^{38}$ und den Ball zuspielten, ${ }^{39}$ pflegte man neben einem Ballholer pro Spieler auch jedem

feriendam conlusoribus praebent, Manil. astr. 5, 165 ff.: ille pilam celeri fugientem reddere planta et pedibus pensare manus et ludere saltu). Vgl. zu dieser nicht befriedigend zu lösenden Frage K. KOCH, Die Geschichte des Fußballs im A/tertum und in der Neuzeit, Berlin 1895, 8 ff.; J. DELANDE, Le football à travers les âges, in: LEC 9, 1940, 410; A. HIRN, Spielten die Griechen Fußball, in: LkE 54, 1935, 479 f. (mit vielen unhaltbaren Thesen); N. YOUNG, Did the Greeks and Romans Play Football?, in: RQ 15, 1944, $310 \mathrm{ff}$.

${ }^{30}$ E. N. GARDINER, $A A W, 230$ : „more probably the youth is bouncing the ball on each thigh alternately."

${ }^{31}$ Vgl. G. LAFAYE, in: DS 4, 1 (1907), s. v. Pila, 477.

32 Sen. epist. 56,1.

33 Ein Wandbild aus den Titusthermen stellt drei junge Männer dar, die unter Aufsicht eines Trainers (Schiedsrichters?) mit je zwei Bällen gleichzeitig spielen, wobei es offen bleibt, ob es um die Zeitdauer oder die höchste Anzahl von Fangbällen geht. Vgl. G. LAFAYE, in: DS 4.1 (1907), s. v. Pila, Fig. 5667; S. MENDNER, Ballspiel, Taf. 13c.

${ }^{34}$ Vgl. G. LAFAYE, in: DS 4,1 (1907), s. v. Pila, Fig. 5668; S. MENDNER, Ballspiel, Taf. 13 a.

35 Quint, inst. 10, 7, 11; Manil, astr. 5, $168 \mathrm{ff.}$

36 Mart. 7, 72, 9; 12, 82, 3; vgl. G. RADKE, in: RE 7 Al (1939), s. v. Trigon, 139; H. A. HARRIS, SGR, 90 ff.; E. WEGNER, Ballspiel, 18, sieht im Trigon den antiken Vorläufer des heutigen Tennis.

37 Isid. 18, 69, 2: „trigonaria est quia inter tres luditur”.

${ }^{38}$ Vgl. S. MENDNER, in: RLAC 10 (1978), s. v. Gesellschaftsspiele, 865. 
Mitspieler einen Diener zu stellen, dessen einzige Aufgabe es war, die Zahl der verpassten Bälle, seines' Spielers zu erfassen ${ }^{40}$.

Beim Mannschaftsspiel ${ }^{41}$ Episkyros $^{42}$ standen sich zwei gleichstarke Teams gegenüber, deren Spielerzahl beträchtlich war ${ }^{43}$. Das Spielfeld war markiert durch eine Mittellinie, auf der der Ball zu Spielbeginn aufgelegt war, und zwei Grundlinien. Ziel des Spieles war es, den Ball über die gegnerische Grundlinie zu bringen. Eine Marmorbasis ${ }^{44}$ aus dem 6. Jh. v. Chr. zeigt uns sechs Spieler - je drei von einer Mannschaft - beim Episkyros ${ }^{45}$. Sehr anschaulich sind die einzelnen Aufgaben der Mitspieler abzulesen ${ }^{46}$. Von der Spielidee und den Rahmenbedingungen ähnlich war das Spiel Phaininda ${ }^{47}$. Bei diesem Spiel,

39 Beim Trigon kam dem Gebrauch der linken Hand eine besondere Bedeutung zu; z. B. Mart. 14, 46: „si me mobilius scis expulsare sinistris, sum tua; tu nescis? rustice, redde pilam!“

40 CIL 4, 1936; vgl. J. P. V. D. BALSDON, Life, 165; V. J. MATTHEWS, Suram dare: $A$ Gesture in Roman Ball Playing, in: Nikephoros 3, 1990, $185 \mathrm{ff.}$

41 R. MANDELL, Sport, 68, spricht dem antiken Sport überhaupt jede Form von Mannschaftssport ab.

42 Poll. 9,104. Vgl. A. MAU, in: RE 6 (1907), s. v. Episkyros, 199 f.; S. MENDNER, Ballspiel, 102 ff. N. YOUNG, in: RQ 15, 1944, 313, vergleicht es mit dem modernen Handball.

43 Hesych. S. v. Éníбкupos.

${ }^{44}$ Vgl. A. PHILADELPHUS, in: JHS 42, 1922, pl. 6; S. CASSON, in: JHS 45, 1925, Fig. 1; L. GRÜNDEL, in: AA 40, 1925, Abb. 5; E. N. GARDINER, AAW, Fig. 212; S. MENDNER, Ballspiel, Taf. 16 a; E. WAGNER, in: Gymnasium 70, 1963, Taf. 24; C. DIEM, Weltgeschichte, Abb. 121; H. A. HARRIS, SGR, pl. 46; F. BREIN, GL 2, Abb. 14; H. M. LEE, in: Stadion 10, 1984, Fig. 7.

${ }^{45}$ Vgl. R. PATRUCCO, Sport, Abb. 167, bezieht die Darstellung fälschlicherweise auf das Spiel Harpastum.

${ }^{46}$ Vgl. die Beschreibungen der einzelnen Spielerfunktionen bei S. MENDNER, Ballspiel, 104 ff.; ferner L. GRÜNDEL, in: AA 40, 1925, 91 ff.

47 Vgl. E. WEGNER, Ballspiel, 33 f.; R. HEUBAUM, in: LkE 58, 1939, 199; S. MENDNER, Ballspiel, 95. Eine völlig wirre Darstellung des Spielablaufs bietet G. LUKAS, Rom, 94. 
das auch Harpastum genannt wurde ${ }^{48}$, war eine rauhere Gangart erlaubt ${ }^{49}$, und der kämpferische Einsatz wurde stärker betont ${ }^{50}$. Doch wie beim Episkyros galt es, den Ball über die gegnerische Grundlinie zu bringen ${ }^{51}$. Beide Ballspielformen weisen eine sehr komplexe Spielweise auf ${ }^{52}$, die in vielen Dingen den heutigen großen Ballspielen kaum nachsteht ${ }^{53}$.

Auch Ballspiele, bei denen spezielle Schlaggeräte benutzt wurden, waren dem antiken Sport nicht fremd. Ein Marmorrelief aus dem 5. Jh. v. Chr. ${ }^{54}$ stellt eine Szene aus einem Hockeyspiel dar ${ }^{55}$. Es zeigt eine verblüffende Ähnlichkeit mit dem heutigen Hockey ${ }^{56}$, was die Spielgeräte Schläger ${ }^{57}$ und

48 Zur Gleichsetzung von Phaininda und Harpastum vgl. Ath. 1,14; ausführlich dazu S. MENDNER, in: Gymnasium 66, 1959, $517 \mathrm{ff}$.; vgl. auch E. WAGNER, Kritische Bemerkungen zum Harpastum-Spiel, in: Gymnasium 70, 1963, 356 f.

49 Galen, de parv. pil. 2 vergleicht es mit dem Ringkampf. Vgl. auch Mart. 7,67,4; Artem. 1,55; Clem. Alex. Paedag. 3,50,1. Vgl. auch Dig. 9,2,52,4 zu einem Beinbruch beim Ballspiel. ${ }^{50}$ So neigen einige Gelehrte zu dem Vergleich mit dem heutigen Rugby so E. N. GARDI NER, AAW, 235; E. WEGNER, Ballspiel, 39.

${ }^{51}$ Vgl. S. MENDNER, in: Gymnasium 66, 1959, 525; dagegen E. HESSEL, in: Gymnasium 67 , 1960, 26.

52 Galen, de. parv. pil. 3; vgl. G. E. MARINDIN, in: CR 4, 1890, 147; E. WENKEBACH, Sudhoffs Arch, Gesch. Med. u. Naturwissensch. 31, 4, 1938, 276.

53 Zu den Anweisungen eines Mitspielers vgl. Athen. 1, 15 a. Zu einer Halbzeit bzw. einem Platzwechsel vgl. E. WAGNER, in: Gymnasium 70, 1963, 365.

${ }^{54}$ Vgl. G. OIKONOMOS, in: AD 6, 1920-1, 57; A. PHILADELPHUS, in: JHS 42, 1922 pl. 7; L. GRÜNDEL, in: Jdl 40, 1925, Abb. 1; B. SCHRÖDER, Sport, Taf. 21; E. N. GARDINER, AAW, Fig. 213; L. DEUBNER, in: Die Antike 6, 1930, Abb. 15; S. MENDNER, Ballspiel, Taf. 16b; C. DIEM, Weltgeschichte, Abb. 123; H. A. HARRIS, SGR, Taf. 47; F. BREIN, GL 2, Abb. 15; V. OLIVOVÁ, Sport, 109.

55 Zum antiken Hockey vgl. Plut. mor. 839c; vgl. E. WAGNER, Hockeyspiel im Altertum, in: Philologus 103, 1959, 137 f.; Zu zwei Statuetten die Hockeyspieler darstellen, vgl. L. GRÜNDEL, in: AA 40, 1925, Abb. 3; L. DEUBNER, in: Die Antike 6, 1930, Abb. 17.

${ }^{56}$ Vgl. L. GRÜNDEL, in: AA 40, 1925, 80; S. MENDNER, Ballspiel, 108; R. PATRUCCO, Sport, 344; anders F. BREIN, GL 2, der meint, dass das dargestellte Spiel nur paarweise 
Ball und die Stellung der Spieler - hier beim Bully ${ }^{58}$ - anbelangt. Aufgabe der Spieler war es, den Ball über eine Linie oder zu einem Mal bzw. Tor zu befördern ${ }^{59}$.

Beim Polo ${ }^{60}$, das in der Spätantike auch auf speziell dafür errichteten Anlagen $^{61}$ ausgetragen wurde, spielten zwei gleich starke Mannschaften mit einem Lederball von der Größe eines Apfels und angemessen langen Stöcken, die in einer breiten Rundung endeten und deren Mittelfläche mit getrockneten Darmsaiten gitterförmig bespannt war ${ }^{62}$. Der Ball lag zu Beginn des Spiels in der Platzmitte, und es galt, ihn mit den Stöcken an das gegnerische Mal zu treiben ${ }^{63}$, was den Sieg bedeutete ${ }^{64}$.

Abschließend sei noch auf die Ballspiele der spartanischen Jugend verwiesen ${ }^{65}$. J edes Jahr trug sie ihre Ballspielmeisterschaften aus. Zwar

durchgeführt wurde und somit nicht mit dem heutigen Mannschaftsspiel Hockey zu vergleichen ist.

${ }^{57}$ Vgl. L. GRÜNDEL, in: JDAl 40, 1925, Abb. 2; W. RUDOLPH, in: Klio 48,1967, 84 f.

${ }^{58}$ Vgl. L. GRÜNDEL, in: JDAl 40, 1925, 82. W.Rudolph,in:Klio 48,1967,85.

59 Vgl. L.GRÜNDEL, in: JDAl 40, $1925,83$.

60 Vgl. C. DIEM, Asiatische Reiterspiele, Berlin 1941, 104 ff., bes. 1201; S. MENDNER, Ballspiel, $110 \mathrm{ff}$. Die Digesten 9, 2, 11, 1 berichten von einer Schadenersatzklage im Zusammenhang mit einem Polospiel. Ein wuchtig geschlagener Ball - hier kann es sich nur um einen kleinen, harten Poloball gehandelt haben - verirrte sich aus dem Spielfeld und traf unglücklichenweise die Hände eines Friseurs, der dadurch seinem Sklaven die Kehle durchschnitt.

${ }^{61}$ Vgl. C. DIEM, Reiterspiele, 121; S. MENDNER, Ballspiel, Taf. 18.

62 Vgl. H. A. HARRIS, SGR, Fig. 83.

63 S. MENDNER, Ballspiel, 112, sieht in diesem Male den Vorläufer des Tores der Moderne.

64 Vgl. L. BECQ DE FOUQUI ERES, Les jeux des anciens, Paris 1869, 186 ff.; S. MENDNER, Ballspiel, 110 ff.; H. A. HARRIS, SGR, 102.

65 Lukian. Anach, 38; M. N. TOD, Teams of Ball-Players at Sparta, in: ABSA 10, 1903-1904,

63 ff.; DERS., Three New LDAIPEIS-Inscriptions, in: ABSA 13, 1906-7, 212 ff.; 
wissen wir nicht, welches Ballspiel praktiziert wurde ${ }^{66}$, doch wir erfahren, dass die lokalen Bezirke Spartas je ein Team stellten. Unter der Führung eines Kapitäns kämpften jeweils 15 Spieler um den begehrten Meistertitel ${ }^{67}$. Die Leitung der Spiele lag in den Händen führender Persönlichkeiten ${ }^{68}$, und Verstöße gegen das Regelsystem wurden empfindlich geahndet ${ }^{69}$. Zahlreiche Inschriften mit ávé $\varphi \varepsilon \delta \rho o \zeta$-Vermerk ${ }^{70}$ belegen, dass man nach einem K.O.Sieg die Siegermannschaft ermittelte ${ }^{71}$.

Auffällige Parallelen zum modernen Sport zeigen sich bei Mannschaftsspielen von der Mannschaftsführung durch den Kapitän über eine Rollenaufteilung und taktisches Spielverhalten bis zur jährlich ausgetragenen Meisterschaft.

A. M. WOODWARD, Inscriptiones Graecae 5, 1: Some Afterthoughts, in: ABSA 43, 1948, 209 ff.; DERS., in: ABSA 46, 191 ff.; H. A. HARRIS, SGR, $103 \mathrm{f}$.

${ }^{66}$ Vgl. S. MENDNER, Ballspiel, 102 f., sieht in Episkyros, da es auch Ephebike genannt wurde, das Spiel der spartanischen jungen Männer.

67 IG 5, 1, 566, 674-87; vgl. M. N. TOD, in: ABSA 10, 1903-1904, 74; DERS., in: ABSA 13, 1906-1907, 217; S. MENDNER, Ballspiel, 96.

68 Paus. 3, 2, 2.

69 Demetr. de. elocut. 122; vgl. J. JÜTHNER, $A L$ 1, 99.

${ }^{70}$ Vgl. M. N. TOD, in: ABSA 10, 1903-1904, 73, vertritt die Ansicht, dass nur für Mannschaften ein Siegesmonument errichtet wurde, die ohne Freilos den Sieg davongetragen hatten.

${ }^{71}$ Vgl. E. N. GARDINER, AAW, 231; H. A. HARRIS, SGR, 103. 
Sowohl aufgrund der geographischen Lage und der klimatischen Verhältnisse, als auch wegen ökonomischer und militärischer Bedingtheiten ist es verständlich, dass der Wassersport bei Griechen und Römern weit verbreitet war und sich allgemeiner Beliebtheit erfreute ${ }^{1}$. Auf der anderen Seite kam dem Wassersport - mit Ausnahme des Ruderns - im Zusammenhang mit Festspielen kaum eine Bedeutung zu, so dass Quellen zum wettkampfmäßigen Schwimmen, Tauchen, Wasserspringen und zu Wasserspielen nur spärlich vorhanden $\operatorname{sind}^{2}$. Das Schwimmen gehörte zu den elementaren Kenntnissen des antiken Menschen ${ }^{3}$, und man kannte schon die Ausprägung verschiedener Schwimmstile, ${ }^{4}$, wobei der Wechselzug mit Beinschlag vorherrschte. ${ }^{5}$ Für Schwimmwettkämpfe im Rahmen organisierter Festspiele haben wir nur einen Nachweis: ${ }^{6}$ Pausanias ${ }^{7}$ berichtet, dass in der

${ }^{1}$ Vgl. E. N. GARDINER, GASF, 507; DERS., AAW, 93; H. A. SANDERS, in: C) 20, 1925, 566; H. BRENDICKE, Zur Geschichte des Schwimmkunst, Hof 1930, 7; B. SCHRÖDER, Sport, 86; R. PATRUCCO, Sport, 351; N. YALOURIS, Games, 259; G. LUKAS, Rom, 101; vgl. auch A. KRÜGER, Schwimmen. Der Wandel in der Einstellung zu einer Form der Leibesübungen, (=Beiträge und Quellen zu Sport und Gesellschaft 2), 1984, 19 ff.

${ }^{2}$ Vgl. I. WEI LER, Sport, $206 \mathrm{f}$.

${ }^{3}$ Plat. leg. 3,689e; Plut. Cato 20; Suet. Aug. 64.

${ }^{4}$ Vgl. E. MEHL, Moderne und antike Schwimmstile, in: Mitt. d. Ver. kl. Phil. Wien 2, 1925, 59 ff.; DERS., Antike Schwimmstile, in: Mitt. d. Ver. kl. Phil. Wien 3, 1926, 85 ff.; DERS., Antike Schwimmkunst, München 1927, 90 ff.; DERS., in: RE Suppl. 5 (1931), s. v. Schwimmen, 853 ff.; H. A. HARRIS, SGR, 120 f.; H. W. PLEKET, GL 2, 286.

${ }^{5}$ E. MEHL, Schwimmkunst, 7; DERS., in: RE Suppl. 5 (1931), s. v. Schwimmen, 847; F. BREIN, GL 2, 127.

6 Für ein allgemein nicht vorhandenes oder unterentwickeltes Wettkampfwesen im Schwimmen sprechen sich aus H. BRENDICKE, Schwimmkunst, 7; E. N. GARDINER, AAW, 93; E. MEHL, Schwimmkunst, 62; DERS., in: RE Suppl. 5 (1931), s. v. Schwimmen, 860; K. SCHÜTZE, in: Hermes 73, 1938, 355; H. A. HARRIS, SGR, 123 f.; N. YALOURIS, Games, 260; F. BREIN, GL 2, 127; G. LUKAS, Rom, 105. Anders R. PATRUCCO, Sport, 351.

${ }^{7}$ Paus. 2,35,1. 
Kaiserzeit ${ }^{8}$ die kleine peloponnesische Stadt Hermione jährlich Preise für einen Schwimm- ${ }^{9}$ und Ruderwettkampf stiftete. Doch hat es mit Sicherheit des Öfteren (spontane und wenig organisierte) Wettschwimmen gegeben ${ }^{10}$. Aus den verstreuten Notizen kann man sich ein ungefähres Bild über den Ablauf solcher Wettschwimmen machen ${ }^{11}$. Trotz der zahlreichen piscinae und Thermen ${ }^{12}$ wurden die Schwimmrennen gewöhnlich in Flüssen oder am Meeresstrand ausgetragen ${ }^{13}$. Eine bestimmte Strecke flussabwärts war zu bewältigen ${ }^{14}$, oder man wetteiferte von einem Ufer zum anderen ${ }^{15}$, die Schwimmstrecke konnte ähnlich der Pferderennbahn mit Start, Wendemarke und Ziel entlang der Ufer verlaufen ${ }^{16}$, oder man schwamm vom Strand ins

8 R. PATRUCCO, Sport, 361 f., geht davon aus, dass sich das Schwimmen erst in der Kaiserzeit von der Arbeitswelt löste und zu einer eigenständigen Sportart wurde.

'Vgl. J. H. KRAUSE, Gymnastik, 633; B. SCHRÖDER, Sport, 87; I. RINGWOOD, Festivals, 63 f.; K. SCHÜTZE, in: Hermes 73,1938, 356 f, und F. BREIN, GL 2, 128 („,vielleicht handelt es

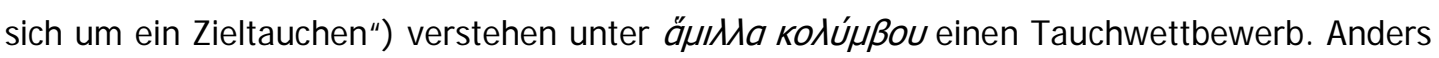
R. PATRUCCO, Sport, 356 n. 2, der sich u. a. aus organisatorisch-technischen Gründen gegen einen Tauchwettbewerb ausspricht. Vgl. auch E. MEHL, in: RE Suppl. 5 (1931), s. v. Schwimmen, 861, wo er ein „langweiliges Dauertauchen“ ablehnt. Vgl. auch noch I. WEI LER, Sport, 209.

${ }^{10}$ Vgl. B. SCHRÖDER, Sport, 86; E. MEHL, Schwimmkunst, 62; H. A. HARRIS, in: PVS 8, 1968-1969, 19; R. PATRUCCO, Sport, 356.

11 Unterschiedliche Wertung der Schwimmkampfdarstellungen des Nonnos; H. A. HARRIS, $S G R, 122$ : "It would be foolish, however, to base any theory on ancient swimming on Nonnus." Dagegen R. PATRUCCO, Sport, 356: „Le descrizioni di Nonno sono indiscutibilmente il trasferimento su piano mitico di un fenomeno reale."

${ }^{12}$ Vgl. D. KRENCKER/E. KRÜGER, Die Trierer Kaiserthermen, Augsburg 1929; R. GINOUVES, Balaneutikè. Recherches sur le bain dans l'antiquité grecque, Paris 1962, 109 ff., E. BRÖDNER, Die römischen Thermen und das antike Badewesen, Darmstadt 1983.

${ }^{13}$ Vgl. H. A. HARRIS, SGR, 118; R. PATRUCCO, Sport, 356; F. BREIN, GL 2, 127.

${ }^{14}$ Hor. carm. 3,7,28.

${ }^{15}$ Nonn. Dion. 11,45.

${ }^{16}$ Nonn. Dion. 11,412 ff. Vgl. dazu die Skizze von H. J. ROSE in seiner Ausgabe des Nonnos, London 1940, 387 (Loeb Classical Library);vgl. R. PATRUCCO, Sport, Fig. 175. 
offene Meer hinaus ${ }^{17}$. Da die Schnelligkeit ${ }^{18}$ der Schwimmer über den Sieg entschied, und wir nichts über eine Festlegung des Schwimmstils lesen, ist es verständlich, dass man eine Art Kraulschwimmstil bevorzugte ${ }^{19}$. Durch Plinius d. J. erfahren wir aber auch von Schwimmwettkämpfen an der Küste Nordafrikas, bei denen neben der Schnelligkeit vor allem die Ausdauer eine entscheidende Rolle spielte ${ }^{20}$. Die Schwimmer wetteiferten, wer am weitesten in das Meer hinaus schwimmen konnte. Sieger war derjenige, der Küste und Konkurrenten am weitesten hinter sich lie $\aleph^{21}$. Schwimmleistungen über bestimmte Distanzen, die meist in Notsituationen unter erschwerten Bedingungen erbracht wurden, sind zahlreich überliefert ${ }^{22}$, doch aus dem agonalen Bereich sind uns weder Zeiten noch bewältigte Schwimmdistanzen bekannt.

Wasserspiele waren den Menschen in der Antike nicht fremd ${ }^{23}$. Doch waren dies kleinere Unterhaltungsspiele und Strandvergnügungen. ${ }^{24}$ Reigenschwimmen und Bilderschwimmen waren eher theatralische Aufführungen $^{25}$, und ein Spiel nach Art des Fischerstechens wurde nicht wettkampfmäßig durchgeführt ${ }^{26}$.

${ }^{17}$ Plin. epist. 9,33.

${ }^{18}$ Hor. carm. 3,7,27; Nonn. Dion. 11,412 ff.; 11,419 f.

${ }^{19}$ Vgl. E. MEHL, Schwimmkunst, 11 u. 40.

${ }^{20}$ Plin. epist. 9,33.

21 Dieser nicht näher bestimmte Austragungsmodus wirft einige Fragen auf. War den Schwimmern ein räumlicher oder zumindest zeitlicher Rahmen gesetzt? Wurde die erzielte Siegesdistanz vermessen? Wie hielt man den Abstand der Schwimmer untereinander fest? Doch die Quellen geben darauf keine befriedigende Antwort.

${ }^{22}$ Vgl. H. N. COUCH, in: CW 39,1945-1946, 34 f.

${ }^{23}$ Vgl. E. MEHL, Schwimmkunst, 104 f.; DERS., in: RE Suppl. 5 (1931), s. v. Schwimmen, $859 \mathrm{f}$.

${ }^{24}$ Gal. de loc. äff. 1,4,8; Plin. epist. 9,33.

25 Sil. Ital. 3,410; Mart, epigr. 26; vgl. G. TRAVERSALI, Gli spettacoli in acqua nel teatro tardo-antico, Rom 1960, $121 \mathrm{ff}$.

${ }^{26}$ Vgl. K. PREISENDANZ, in: RE 14,1 (1928), s. v. Maiumas, $620 \mathrm{ff.}$ 
Das Wasserspringen diente zum Schwimmstart und zum Erreichen einer größeren Tauchtiefe ${ }^{27}$. Doch wurden Wassersprünge verschiedener Art $^{28}$ von erhöhten Positionen wie Felsen ${ }^{29}$ oder eigens dafür errichteten Sprungtürmen ${ }^{30}$ durchgeführt. Es ist naheliegend, dass es Veranstaltungen gab, bei denen die Sprünge bewertet wurden und die Teilnehmer einer Rangliste unterlagen ${ }^{31}$.

Für das Tauchen lässt sich ebenfalls eine wettkampfmäßige Durchführung nicht zweifelsfrei nachweisen ${ }^{32}$. Tauchleistungen, die im Zusammenhang mit kriegerischen Ereignissen oder von berufsmäßigen Tauchern uns mitgeteilt werden $^{33}$, sind wenig konkret ${ }^{34}$ oder völlig unglaubwürdig ${ }^{35}$. Aus dem sportlichen Bereich liegen uns keine Leistungsnachweise vor.

\footnotetext{
${ }^{27}$ Vgl. E. MEHL, Schwimmkunst, $106 \mathrm{ff}$.

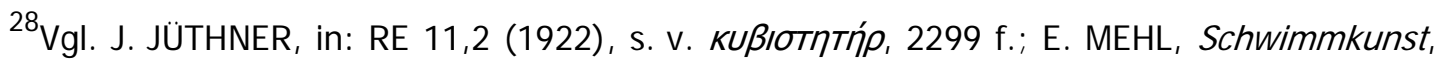
107 f. Anders F. BREIN, GL 2, 127.

${ }^{29}$ Vgl. E. MEHL, Schwimmkunst, Fig. 10; E. N. GARDINER, AAW, Fig. 62; M. PALLOTTINO, La peinture étrusque, Genf 1952, 50; R. PATRUCCO, Sport, Fig. 170. Zu den Sprüngen vom etwa $60 \mathrm{~m}$ hohen leukadischen Felsen vgl. J. H. KRAUSE, Gymnastik, 633.

${ }^{30}$ Vgl. M. NAPOLI, Le pitture greche della tomba del tuffatore, in: Le Scienze, April 1969, 9 ff.; R. PATRUCCO, Sport, 355 und Fig. 174; N. YALOURIS, Games, Fig. 152. Vgl. aber auch W. J. SLATER, High Flying at Paestum, in: AJA 80, 1976, 423 ff. u. AJ A 81, 1977, 555 ff.; R. R. HOLLOWAY, High Flying at Paestum. A Reply, in: AJ A 81, 1977, 554 u. Fig. 1.

${ }^{31}$ Gegen ein wettkampfmäßiges Wasserspringen sprechen sich aus E. MEHL, in: RE Suppl. 5 (1931), S. v. Schwimmen, 854; F. BREIN, GL 2, 127 f.; I. WEILER, Sport, 209. Vgl. aber PATRUCCO, Sport, 355.

32 Vgl.B.SCHRÖDER,Sport,86; R.PATRUCCO, Sport, 356.

${ }^{33}$ Vgl. E. MEHL, Schwimmkunst, 111 ff.; DERS., in: RE Suppl. 5 (1931), s. v. Schwimmen,

854 f.; F. J. FROST, Scyllias: Diving in Antiquity, in: G \& R 15, 1968, 180 ff.

34 Sen. de ira 2,3 .

${ }^{35}$ Hdt. 8,8; Opp. hal. 1,84.
} 
Für den Segelsport bietet Catull den einzigen Beleg ${ }^{36}$. Doch diese wenig ergiebige Quelle erwähnt nur die Unbesiegbarkeit des Bootes, gibt aber keine Details über Regeln oder den Ablauf eines Rennens ${ }^{37}$. Dafür haben wir relativ umfangreiches Material zum Rudersport. Ruderregatten fanden mit und ohne Bezug zu Götter- und Heroenfesten an verschiedenen Orten und bei verschiedenen Festen statt und wurden besonders von Epheben betrieben ${ }^{38}$. Die bei den Rennen verwendeten Boote scheinen zumindest in späterer Zeit ausschließlich dem Rennrudern gedient zu haben ${ }^{39}$. Wie stark die Besatzung (gewöhnlich mit Steuermann) gewesen ist, lässt sich nicht eindeutig bestimmen ${ }^{40}$. Bildliche Darstellungen von Ruderbooten mit Mannschaft, die eindeutig dem Rennrudern zuzuordnen sind, zeigen zwischen drei $^{41}$, fünf ${ }^{42}$ und $a \mathrm{ach}^{43}$ Besatzungsmitglieder. Ob dies der künstlerischen Freiheit entsprang ${ }^{44}$ oder man schon Rennen verschiedener Bootsklassen kannte,

\footnotetext{
${ }^{36}$ Catull. 4,2 ff.
}

37 Vgl. H. A. HARRIS, SGR, 126.

${ }^{38}$ Vgl. P. GARDNER, Boat-Races among the Greeks, in: JHS 2, 1981, 90ff.; DERS., BoatRaces at Athens, in: JHS 2, 1881, 315 ff.; DERS., A Stele Commemorating a Victory in a Boat-Race, in: JHS 11, 1980, 146 ff.; A. MOMMSEN, Feste der Stadt Athen, Leipzig 1898, 145 ff.; L. DEUBNER, Feste, 174, 205, 215, 223, 228; H. A. HARRIS, SGR, 127 ff; R. PATRUCCO, Sport, 357 ff., V. OLIVOVÁ, Sport, 130; D. J. KYLE, Athens, 37 u. 193.

39 P. GARDNER, in: JHS 11, 1890, 150; L. DEUBNER, Feste, 205; R. PATRUCCO, Sport, 358; N. YALOURIS, Games, 262. G. LUKAS, Rom, 110, geht davon aus, dass gewöhnlich mit einem Blatt gerudert wurde.

${ }^{40}$ Vgl. F. BREIN, GL 2, 128.

${ }^{41}$ Vgl. E. N. GARDINER, AAW, Fig. 66; R. PATRUCCO, Sport, Fig. 177.

${ }^{42}$ Vgl. E. N. GARDINER, $A A W$, Fig. 68; B. SCHRÖDER, Sport, Taf. 45; R. PATRUCCO, Sport, Fig. 178.

${ }^{43}$ Vgl. P. GARDNER, in: JHS 11, 1890, Fig. 1 u. 2; E. N. GARDINER, GASF, Fig. 190; DERS., AAW, Fig. 67; R. PATRUCCO, Sport, Fig. 176. Im oberen Teil der Stele sind weitere drei Personen bei der Siegerehrung dargestellt; vgl. dazu P. GARDNER, in: JHS 11, 1890, 146.

${ }^{44}$ Vgl. E. N. GARDI NER, AAW, 95. 
bleibt offen ${ }^{45}$. Wir erfahren auch nichts über die Renndistanz ${ }^{46}$, wobei die Rennen im Meer, aber auch in dafür geeigneten Flüssen stattfinden konnten ${ }^{47}$. In Anlehnung an Vergils Darstellung ${ }^{48}$ ist der Ablauf eines Rennens gut nachzuvollziehen ${ }^{49}$. Start und Ziel waren am Ufer ${ }^{50}$. Die Vergabe der Startpositionen erfolgte durch $\operatorname{Los}^{51}$. Das Startzeichen gab ein Tubabläser $^{52}$, als Rennstrecke diente eine Doppelbahn mit Wendemal ${ }^{53}$. Sieger war das Boot, das als erstes das Ziel erreichte ${ }^{54}$. Die Sieger wurden durch den Herold ausgerufen ${ }^{55}$. Es folgte die Siegerehrung ${ }^{56}$ mit stattlichen Preisen für Sieger und Platzierte ${ }^{57}$ und sogar einem Trostpreis für den

45 Zu Ruderbooten vgl. G. S. KIRK, Ships on Geometric Vases, in: ABSA 44, 1949, 93 ff; J. S. MORRISON/R. T. WILLIAMS, Greek Oared Ships 900-322 B. C., Cambridge 1968; L. CASSON, Ships and Seamanship in the Ancient World, London 1971; A. F. TILLEY/V. H. FENWICK, Rowing in the Ancient Mediterranean, in: Mariner's Mirror, 1973, $96 \mathrm{ff}$; J. PEKÄRY, Vorarbeiten zum Corpus der hellenistisch-römischen Schiffsdarstellungen, in: Boreas 7, $1984172 \mathrm{ff}$.

${ }^{46}$ Anders P. GARDNER, in: JHS 2, 1881, 316; vgl. aber L. ZIEHEN, in: RE 18,3 (1949), s. v. Panathenaia, 486.

${ }^{47}$ Vgl. R. PATRUCCO, Sport, 358 n. 2.

${ }^{48}$ Aen. 5,106 ff.

${ }^{49}$ Vgl. G. STEGEN, Des regates dans l'Eneide, in: Latomus 27, 1968, 600 ff.; H. A. HARRIS, SGR, 128 ff.; N. YALOURIS, Games, 262; I. WEI LER, Sport, 208 f.; G. LUKAS, Rom, 110.

50 Verg. Aen. 5, $129 \mathrm{ff}$.

${ }^{51}$ Verg. Aen. 5,132.

52 Verg. Aen. 5,138.

${ }^{53}$ Verg. Aen. 5,139 ff. Vgl. die Skizze bei H. A. HARRIS, SGR, Fig. 5.

${ }^{54}$ Verg. Aen. 5,240 ff. Die dafür benötigte Zeit war - wie auch heute - ohne Belang.

${ }^{55}$ Verg. Aen. 5,245.

${ }^{56}$ Verg. Aen. 5,246.

57 IG 2/32, 2311=Syll.3 1055; vgl. aber L. ZEHEN, in: RE 18,3 (1949), s. v. Panathenaia, 485 f.; S. G. MI LLER, Arete, 47; D. J. KYLE, Athens, 37. Zu Preisen bei Ruderrennen vgl. Lys. 21,5; Arr. anab. 7,23,5; Paus. 2,35,1. 
Letzten $^{58}$. Die von Vergil gebotene Schilderung eines Ruderrennens war zweifelsohne an zeitgenössische Darbietungen angelehnt ${ }^{59}$ und zeigt die Gestaltung eines wohlorganisierten Ruderwettkampfwesens, das alle Voraussetzungen zur objektiven Erfassung der sportlichen Leistung erfüllte.

58 Verg. Aen. 5,282 f. Dem hofffnungslos abgeschlagenen Sergestus muss es nach der Zerstörung der Riemen auf der einen Seite erlaubt gewesen sein, die Segel zu setzen. ${ }^{59}$ Vgl. I. WEI LER, Sport, 208. 
Für das Gewichtheben als eigenständige sportliche Disziplin und als Konkurrenz im Rahmen von Festveranstaltungen fehlen uns eindeutige Beweise ${ }^{1}$. Dennoch wissen wir, dass sich Menschen in der griechischrömischen Antike in Kraftakten mit großen Gewichten gemessen oder darin ihre physische Stärke und Leistungsfähigkeit unter Beweis gestellt haben², wobei einzigartige Leistungen erreicht wurden. Ein noch umfangreicheres Feld der Beschäftigung mit unterschiedlich schweren, z. T. genormten Objekten finden wir beim Training der Leicht- und besonders der Schwerathletik ${ }^{3}$. Als Übungsgeräte konnten die Sprunggewichte Verwendung finden, ${ }^{4}$ die aber aufgrund ihres geringen Gewichtes ${ }^{5}$ nur eine unbedeutende Kraftzunahme bewirkten und eher einer stärkenden gymnastischen Übung entsprachen ${ }^{6}$. Das Heben mittelgroßer Steine, wie es auf einer rotfigurigen Schale des Würzburger Museums zu sehen ist ${ }^{7}$, stellt Training mit Gewichten dar, das als gezieltes Krafttraining mit entsprechender Trainingseffizienz

\footnotetext{
${ }^{1}$ Vgl. E. MEHL, in: RE 7 A2 (1948), s. v. Turnkunst, 2520; H. A. HARRIS, SGR, 142 f.; L. MORETTI, IAG, 4 f.; W. DECKER, Sport, 147: ,... das Gewichtheben, das bei keinem antiken Agon als Wettkampf ausgeschrieben war."

2 Vgl. Philostr. gymn. 43: Tragen schwerer Lasten, Verbiegen von Eisenplatten, Kräftemessen mit Tieren, u. ä.; E. MEHL, in: RE 7 A2 (1948), s. v. Turnkunst, 2548; E. N. GARDINER, AAW, 54; DERS., GASF, $82 \mathrm{f}$.
}

${ }^{3}$ Vgl. N. B. CROWTHER, Weightlifting in Antiquity:Achievement and Training, in: G \& R 24,1, 1977, 115 f.; Epikt. 3,12,9; 3,20,10: Beliebtheit des Gewichthebens. Vgl. J. P. V. D. BALSDON, Life, 161. J uv. 4,419: Training mit Gewichten von Frauen.

${ }^{4}$ Vgl. E. N. GARDI NER, AAW, Fig. 105; J. JÜTHNER, Turngeräthe, Fig. 13; 16;DERS. $A L 2$, Taf. 64.

${ }^{5}$ Vgl. J. JÜTHNER, $A L$ 2; 239 u. 241.

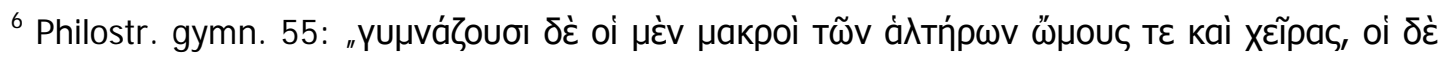

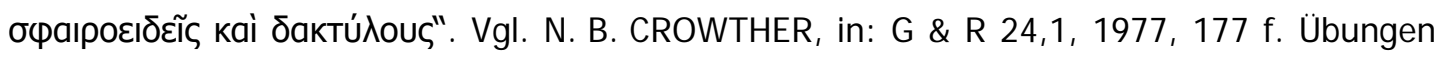
mit leichten Gewichten: Oreib. 6,14,34; Gal. san. ruenda 2,11 3-Epikt. 1,4,13 f.

${ }^{7}$ Vgl. E. LANGLOTZ, Griechische Vasen, München 1932, Nr. 476, Fig. 153; J. JÜTHNER, AL 1, Taf. 5c; C. DIEM, Weltgeschichte, Abb. 114; R. PATRUCCO, Sport, Fig. 51; H. A. HARRIS, GAA, Fig. 30b: „Athlete cleaning Stadium“. 
anzusehen ist ${ }^{8}$. Von einem Satz normierter Gewichte berichtet ein Epitaph aus Dalmatien: "Hic lapide lusit ponderibus his $X X X X, L, C^{\prime 9}$. Versteht man ludere als ein Synonym für exercere, so handelt es sich hierbei um Gewichttraining und nicht um "some kind of juggling performance ${ }^{\mu 10}$. Erfahren wir aus der oben angeführten Inschrift die genauen Gewichtsangaben der Übungsgeräte, so gibt uns Hieronymus Auskunft über die Trainingsformen:
"usque hodie per omnem Judaeam vetus consuetudo servatur, ut in viculis, oppidis et castellis rotundi ponantur lapides gravissimi ponderis, ad quos iuvenes exercere se solent et eos pro varietate virium sublevare, alii usque ad genua, alii usque ad umbilicum, alii ad humeros et caput; nonnulli supra verticem, rectis iuntisque manibus, magnitudinem virium demonstrantes pondus extollunt"11.

Auch berichtet Hieronymus, dass das Heben einer äußerst schweren eisernen Kugel in Athen als Qualifikation zu den Agonen diente ${ }^{12}$. Von einem

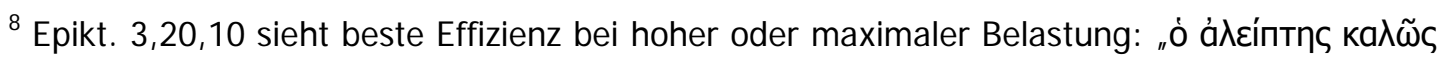

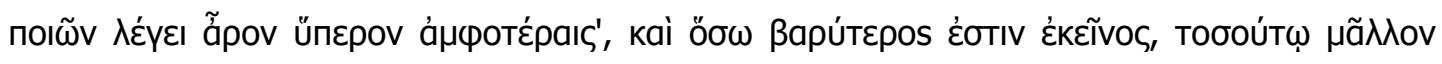

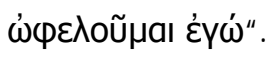

${ }^{9}$ ClL III Suppl. 12924=ILS 5174a.

${ }^{10}$ Vgl. H. A. HARRIS, SGR, 150; vgl. N. B. CROWTHER, in: G \& R 24.1, 1977, 117.

11 Ad Zach 3,12,896; vgl. DERS., eccl. 6,22. Ein weiterer Beleg für das Heben über

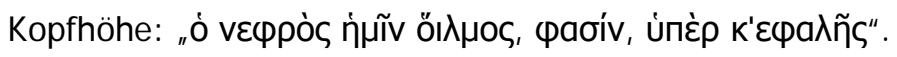

12 Ad Zach. 3,12,896 f.: „in arche Atheniensium iuxta simulacrum Minervae vidi sphaeram aheneam gravissimi ponderis, quam ego pro imbecillitate corpusculi mei moverei vix potui. cum autem quaererem, quid sibi vellet, responsum est ab urbis eius cultoribus athletarum in illa massa fortitudinem comprobari nec prius ad agonem quemquam descendere, quam ex levatione ponderis sciatur, quis cui debeat comparari“. Vgl. E. MENDNER, Ballspiel, 79. E. MEHL in: RE 7 A2 (1948), s. v. Turnkunst, 2534, glaubt, dass entsprechend der Leistung mit der Kugel verschiedene Gruppen, ähnlich unseren Gewichtsklassen, gebildet wurden,
} 
systematischen, allerdings auch sehr ausgefallenen Krafttraining des erühmten Athleten Milo berichtet Quintilian: „Milo, quem vitulum assueverat feaurum ferebat ${ }^{\text {t3 }}$, was er auch in Olympia mit einem vier J ahre alten Stier auf den Schultern demonstrierte. ${ }^{14}$ Trotz dieser beachtlichen Leistung wurde Milo von dem Hirten Titormus übertroffen, der einen gewaltigen Felsbrocken, den Milo kaum bewegen konnte, bis zur Schulter hob, inn etwa $14 \mathrm{~m}$ weit trug und dann warf ${ }^{15}$. Was das Stoßen von Steinen bzw. Kugeln anbelangt, so wissen wir, dass es solche Konkurrenzen in Zusammenhang mit anderen athletischen Wettkämpfen gab ${ }^{16}$. Ein Sandstein im Museum zu Olympia mit den Maßen 0,33 m x 0,68 m x 0,39 m sowie einem Gewicht von 143,5 kg

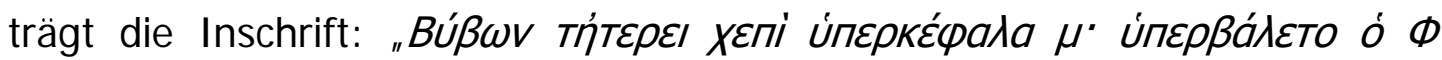
... “. ${ }^{17}$ Mag die Technik des Stoßes nicht eindeutig zu klären sein ${ }^{18}$, so können

wobei die Höhe, bis zu der die Kugel gehoben wurde, oder die Anzahl der Hebungen gewertet wurde.

${ }^{13}$ Quint, inst. 1,9,5.

14 Ath. 10,412e-f, der Theodorus (FHG 4,513) zitiert. Vgl. N. B. CROWTHER, in: G \& R 24.1, 1977, 114: ,... we can estimate that he was carrying a weight of approximately $500-550 \mathrm{~kg}$, to judge from present-day livestock." E. MEHL, in: RE 7 A2 (1948), s. v. Turnkunst, 2534, schätzt bei der sehr kleinen Rinderrasse das Gewicht des Stieres auf 250-300 kg.

${ }^{15}$ Ael. var. hist. 12,22.; vgl.N. B. CROWTHER, in: G \& R 24.1, 1977, 114: „... Titormus probably pushed the weight away from his body, rather than jerked it overhead." Das Gewicht des Steines ist nicht angegeben, dafür aber die Distanz, über die er getragen wurde: „हैф

${ }^{16}$ E. PREUNER, Griechische Siegerlisten, in: MDAI (A), 28, 1903, 366 (Liste vier am Ende). Bronzestatuette aus dem Museo Civico in Bologna: B. SCHRÖDER, Diskobol, Taf. 10; DERS., Sport, Taf. 46a; C. DIEM, Weltgeschichte, Abb. 117; N. YALOURIS, Games, 191.

17 IvO 5, 717; Syll.3 1071; vgl. L. MORETTI, IAG, Nr. 2. Zu rein philologischen Problemen dieser Inschrift vgl. J. ZINGERLE, Commentationes Vindobonensis 3, 1937, 11 f. Es scheint nicht ungewöhnlich, dass der Stein, mit dem die überragende Leistung erbracht wurde, mit einer Inschrift versehen der Gottheit geweiht wurde, wie es oft mit Sprunggewichten und Disken geschah, vgl. H. A. HARRIS, SGR, 145; J. JÜTHNER, Turngeräthe, 23; E. MEHL, in: RE 7 A2 (1948), s. v. Turnkunst, 2534.

${ }^{18}$ Vgl. N. B. CROWTHER, in: G \& R 24.1, 1977, 112: „Bubon seems to have 'thrown' the boulder with one hand over his head, above his head, or head-height." 
wir doch bei dem objektiv zu messenden Gewicht des Steines hierin eine beeindruckende, eine rekordverdächtige Leistung sehen ${ }^{19}$, an deren Wahrheitsgehalt bisweilen gezweifelt wird ${ }^{20}$. Von der Mehrheit der Gelehrten wird die Leistung Bybons jedoch in Frage gestellt ${ }^{21}$, und F. MEZÖ hat die Möglichkeit eines Wurfes mit solch einem Gewicht durch Vergleich mit neuzeitlichen Leistungen unterstrichen ${ }^{22}$, N. B. CROWTHER mittels überlieferter imposanter Kraftleistungen des Mittelalters und der Neuzeit und unter Hinzuziehen aktueller Weltbestleistungen sowohl das einarmige Heben wie Stoßen eines solchen Steines als "perfectly possible" nachweisen können ${ }^{23}$.

Über die Leistung des Hermodikos von Lampsakos sind wir durch zwei

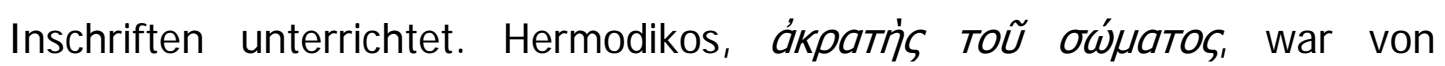

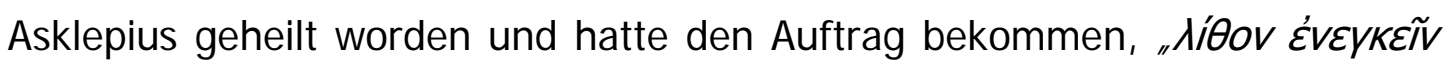

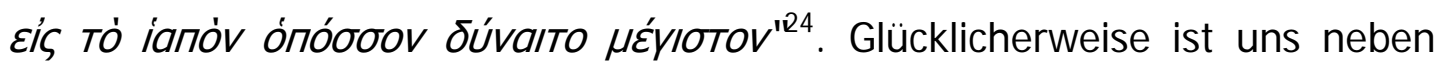
dieser Inschrift aus dem Asklepius-Heiligtum von Epidaurus auch der Stein mit Bestätigung der Ausführung der göttlichen Weisung durch den

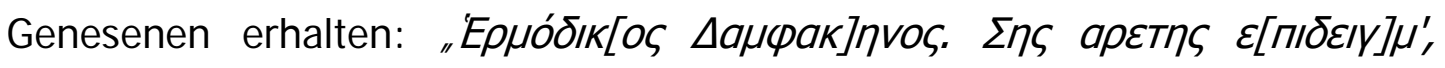

19 Ebd. 112 f. Vgl. IvO 5, 718: ' die Maße: Höhe links 0,42 m, rechts 0,37 m; Breite 0,34 m; Dicke 0,17 m; Gewicht ca. $45 \mathrm{~kg}$. Hierbei geben nicht Größe und Gewicht Zweifel, sondern die philologische Unsicherheit, inwieweit $\rho ı п \rho \rho$ abgeleitet ist von ist; vgl. IvO 5, 719.

${ }^{20}$ L. MORETTI, IAG, 6: ,... in practica non e possibile afferrare, e tanto meno sollevare, con una sola mano il masso di Bybon." E. N. GARDINER, in: JHS 27, 1907, 2, und GASF, 83, glaubt, dass Bybon den Stein mit beiden Händen zur Hochstrecke gebracht, ausbalanciert und dann einarmig gestoßen hat. H. A. HARRIS, SGR, 144 f., hält den Stein aufgrund einer Aushöhlung für "an unfinished cleat for a rope“ und versteht die Inschrift als rein satirisch.

${ }^{21}$ Vgl. B. SCHRÖDER, Sport, 96; F. MEZÖ, Geschichte, 127; J. JÜTHNER, AL 1, 84 f.; R. PATRUCCO, Sport, 145 n. 3; N. YALOURIS, Games, 255.

${ }^{22}$ Vgl. F. MEZÖ, Geschichte, 127; R. GASCH, Volkstümliche Wettübungen, Leipzig 1906, 169.

${ }^{23}$ Vgl. N. B. CROWTHER, in: G \& R 24, 1, 1977, $122 \mathrm{f}$.

24 IG 4, 951, 109 f. 


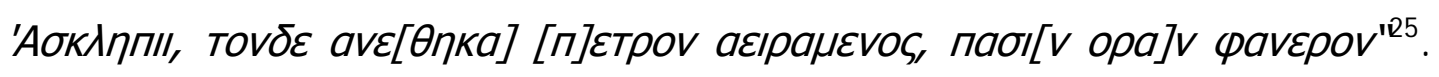
Da sich das Gewicht des Steines auf $334 \mathrm{~kg}$ beläuft, wird von vielen die Inschrift als Fälschung und/oder die Leistung als unmöglich angesehen ${ }^{26}$. Versteht man aber $\varphi \varepsilon \rho \omega$ als bringen oder herbeischaffen, so wäre ein

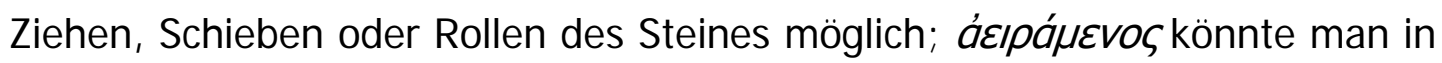
Anlehnung an ein Vasenbild des Ashmolean Museum ${ }^{27}$ das Heben des Steines, der mit den Maßen 1,24 m x 0,37 m x 0,28 m einem dicken Pfosten gleicht, so verstehen, dass der Stein nur an einem Ende gehoben und über das am Boden verbliebende Ende bewegt wurde.

Was das alleinige Gewichtheben anbelangt, so finden wir in dem dem Eumastas zugeschriebenen Felsbrocken ein beeindruckendes Zeugnis:

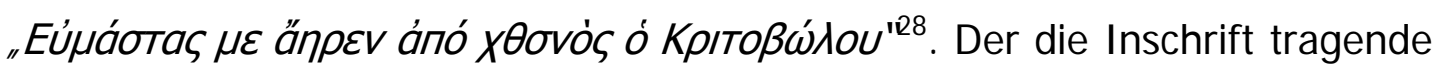
Stein von der Insel Thera hat einen Umfang von 2,18 $\mathrm{m}$ und 1,90 $\mathrm{m}$ und ein Gewicht von $480 \mathrm{~kg}$. Dass es Versuche oder Wettkämpfe gab, gewaltige Felsbrocken zu heben, ist uns durch mehrere Vasenbilder überliefert, wobei die dargestellten Felsbrocken z. T. in Umfang und Gewicht in etwa dem des Eumastas entsprachen ${ }^{29}$. H.A.HARRIS und L.MOREITI halten die Leistung

\footnotetext{
25 IG 4, 954.
}

${ }^{26}$ R. HERZOG, Die Wunderheilung von Epidaurus, in: Philologus Suppl. 22, 1931, 100 ff., hält die Inschrift aus stilistischen wie inhaltlichen Gründen für eine Fälschung. N. B. CROWTHER, in: G \& R 24.1, 1977, 11, vermutet, dass der von Hermodikus tatsächlich bewegte Stein kleiner war. H. A. HARRIS, SGR, 148, ist zu spekulativ, wenn er schreibt, „and it is possible that Hermodicus was persuaded under hypnotism that he really had carried the stone into the temple."

${ }^{27}$ Vgl. J. D. BEAZLEY, in: AJA 43, 1939, 618-620, Taf. 11; Ch. SOURVINON-INWOOD, Theseus Lifting the Rock and a Cup near the Pinthos Painter, in: JHS 91, 1971 Taf. 12b.

28 IG 12,3,449; vgl. G. PFOHL, Griechische Inschriften, Tübingen, Nr. 149.

${ }^{29}$ Vgl. E. POTTIER, Vases antiques du Louvre, Paris 1901, Bd. 2, Taf 9; E. N. GARDINER, in: JHS 27, 1907, Fig. 1; B. SCHRÖDER, Sport, Abb. 19; R. PATRUCCO', Sport, Fig. 52. C. H. E. HASPELS, Attic Black-Figured Lekythoi, Paris 1936, 140 n. 2; Taf. 79,5 und 80,2; P. ZANCANI MONTUORO, La pena di Sisifo, in: Memorie della società Magna Grecia NS 5 , 1964, 60-70, Taf. 13 f.; Ch. SOURVINON-INWOOD, in: JHS 91, 1971, Taf. 12. 
des Eumastas für unmöglich. Ersterer glaubt, dass die Inschrift in Anlehnung an die Bybons verfasst sei und als Scherz eines Mitbürgers des Eumstas aufzufassen ist; letzterer hält es für unmöglich, einen Stein dieses Umfangs zu umfassen und zu heben, und gibt folgende Erklärung:

\begin{abstract}
„Può pensarsi quindi o che essi sollevassero in effetti pesi inferiori e più maneggevoli delle pietre iscritte a noi pervenute e che facessero poi incidere il ricordo su pietre più grandi e pesanti, overo che le loro affermazioni altro non siano che una bravata, senza alcun rapporto con imprese realmente condotte a termine. $^{\text {, }}$
\end{abstract}

Da die Inschrift aber nicht besagt, wie hoch der Stein bewegt wurde, konnte schon die geringste Fortbewegung des Steins Anlass zu dieser Inschrift gewesen sein. Solch eine Leistung, zumal ohne einschränkende Auflagen durch ein Regelwerk, ist also durchaus möglich ${ }^{31}$.

Anhand der angeführten Beispiele ist zu ersehen, dass im Bereich des Gewichthebens ein besonders starkes Interesse bestand, brillante Leistungen zu erzielen und mit gebührendem Stolz zu vermerken.

\footnotetext{
${ }^{30}$ Vgl. L. MORETTI, IAG, 6; H. A. HARRIS, SGR, 146.

${ }^{31}$ Vgl. N. B. CROWTHER, in: G\& R 24.1, 1977, $111 \mathrm{f}$.
} 
$2.5 \quad$ Bogenschießen

Das Bogenschießen ist zwar nicht Bestandteil der großen sportlichen Wettkämpfe gewesen, hat aber in den Leibesübungen der Epheben ${ }^{1}$ und bei kleineren Sportveranstaltungen seinen Platz gehabt ${ }^{2}$. In Bezug auf das sportliche Bogenschießen kannte man Weit- und Zielschießen ${ }^{3}$. In der Antike gab es Bogenschießwettbewerbe, bei denen allein die erzielte Weite über den

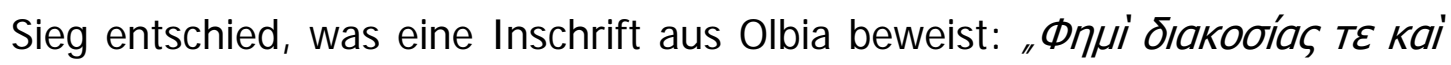

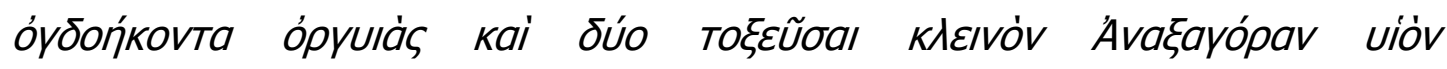

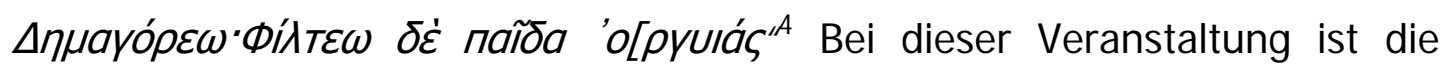

${ }^{1}$ Plat. leg. I,625d; 7,794d f.; 7,813d; 8,834d; Aristot. Ath. pol. 42,3; Syll.3 958; 1059-1062; vgl. W. DITTENBERGER, De ephebis Atticis, Diss. Göttingen, 1863, 34 u. 54; A. DUMONT,

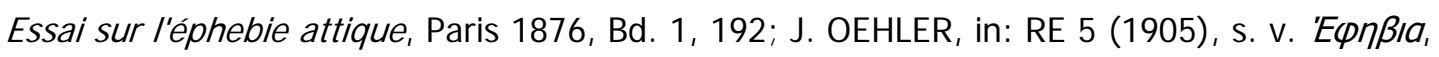
2738; M. W. TECHNAU, in: MDAl (R) 46, 1931, 189 ff. Taf. 17 u. 191 Abb. 1; L. ROBERT, Étud. anat., 399 ff.; J. JÜTHNER, AL 1, 107; R. TÖLLE-KASTENBEIN, Pfeil und Bogen im antiken Griechenland, Bochum 1980, Taf. 4 u. 6.

${ }^{2}$ Syll.3 578; I. WEILER, Sport, 197, spricht von "Mauerblümchendasein" des Bogenschießsportes.

${ }^{3}$ Vgl. R. TÖLLE-KASTENBEI N, Pfeil, 35, führt noch im Rahmen des Bogensports den Wettlauf mit voller Bogenausrüstung an (Plat. leg. 833b); vgl. ebd. Taf. 12 die Darstellung eines schussbereiten Bogenschützen, der auf dem Rücken eines Pferdes steht, was von der Autorin in Beziehung zu der Übung der Apobaten gebracht wird und zu einem Vergleich mit der Disziplin des Biathlon führt. Nicht in den sportlichen Bereich gehört die Darstellung einer Gauklerin, die den Bogen mit den Füßen spannt; B. SCHRÖDER, Sport, Taf. 110; R. TÖLLEKASTENBEIN, Pfeil, Taf. 8; Über Bogen, Pfeile und Schießtechnik vgl. M. BUCHNER, Das Bogenschießen der Aegineten, in: Ztschr. f. Ethnologie 40, 1908, H. 6, 845 ff.; A. SCHAUMBERG, Bogen und Bogenschießen bei den Griechen, Diss. Erlangen 1910, bes. 54 ff.; E. BULANDA, Bogen und Pfeil bei den Völkern des Altertums, in: Abh. arch. epigr. Sem. d. Univ. Wien 15, 1913, 52 ff.; H. MILTNER, in: RE 6,2 (1937), s. v. Tó̧ov, 1847 ff.; R. TÖLLE-KASTENBEIN, Pfeil, $11 \mathrm{ff}$.

${ }^{4}$ Vgl. E. v. STERN, Der Pfeilschuß des Olbiopoliten Anaxagoras, in: JÖAl 4, 1901, (Beiblatt), 57 f.; E. BULANDA, Bogen, 126; L. MORETTI, IAG, 82 ff.; W. MCLEOD, The Range of the Ancient Bow, in: Phoenix 19.1, 1965, 6; R. TÖLLE-KASTENBEIN, Pfeil, 34. B. SCHRÖDER, Sport, 156, führt in diesem Zusammenhang den Begriff des Rekordes an, was aus der Quelle nicht hervorgeht. 
erzielte Weite nicht nur durch eine einfache Markierung festgehalten worden, sondern man hat sie genau vermessen: 282 Klafter $=501,7 \mathrm{~m}^{5}$. Auch die Weite des Zweitgenannten muss gemessen worden sein, doch die Angabe darüber ist verderbt ${ }^{6}$. Diese durch den berühmten Anaxagoras erzielte enorme Weite ist schon vom Herausgeber der Inschrift als durchaus erzielbar hingestellt worden ${ }^{7}$. Die erzielten Schussweiten, die uns aus der Antike überliefert sind, bewegten sich in etwa bei einem Drittel der von Anaxagoras erreichten $^{8}$; dabei ist zu bedenken, dass es sich meist um den militärischen Einsatz von Pfeil und Bogen handelte, wobei eine gewisse Durchschlagskraft der Pfeile nötig war'. Im sportlichen Wettkampf dagegen waren Pfeil und Bogen speziell für den Zweck gewählt worden, eine möglichst große Weite zu erreichen ${ }^{10}$.

${ }^{5}$ Vgl. E. v. STERN, in: J ÖAl 4, 1901, 58, n. 2; E. BULANDA, Bogen, 126 n. 6.

6 Im Allgemeinen vertritt man die durch nichts belegte Meinung, dass der Zweitgenannte auch der Zweitplazierte war; ebenso gut ist eine umgekehrte Folgerung denkbar.

${ }^{7}$ Vgl. E. v. STERN, in: JÖAl 4, 1901, 58 ff., führt Leistungen aus dem Mittelalter und der Neuzeit an, die weit höher sind; vgl. auch L. MORETTI, IAG, $82 \mathrm{ff}$.

${ }^{8}$ Anders P. MEDINGER, L'are turquois, in: RA 6. Ser. 2, 1933, 232: „Pour un archer parthe je crois pouvoir évaluer la portée de 400 à 600 mètres, selon la force physique de l'homme." Eine gute Zusammenstellung der antiken Quellen bezüglich erzielter Schussweiten bei W. MCLEOD, in: Phoenix 19.1, 1965, 7 ff.; vgl. DERS., The Bowshot and Marathon, in: JHS 90, 1970, 197 f.; R. TÖLLE-KASTENBEIN, Pfeil, 35.

9 Im sportlichen Bereich spielte die Durchschlagskraft der Pfeile keine Rolle wie etwa beim Bogenschießen der Pharaonen, vgl. W. DECKER, Die physische Leistung Pharaos, Köln 1971, 84 ff.; W. BURKERT, Von Amenophis I. zur Bogenprobe des Odysseus, in: GB 1, 1973, 72 ff. Lukian. Herrn. 33 berichtet, dass die Skythen die Kraft ihrer Bogenschüsse an mit frischer Rindshaut überzogenen Schilden mit Holzscheiben prüften und ein Durchdringen dieser Ziele mit dem Durchschießen einer Rüstung gleichsetzten. Cass. Dio 62,3,2 erzählt, dass Tiridates, ein Gast des Kaisers Nero, mit einem Pfeil zwei Stiere zugleich tötete.

${ }^{10}$ Vgl. W. McLEOD, in: Phoenix 19.1, 1965, 7: „The stele of Anaxagoras records a "flight shot", made with a strong bow, a light arrow, a high angle of projection, and no assigned target." 
Hinsichtlich des Zielschießens finden sich die unterschiedlichsten beweglichen und festen Ziele ${ }^{11}$. In der Odyssee gilt es, die Ösen von zwölf Doppeläxten zu durchschießen ${ }^{12}$, bei den Patroklosspielen eine an einer Schnur angebundene Taube $\mathrm{zu}$ treffen $^{13}$. Eine Art Zielscheibe sieht man auch auf einer schwarzfigurigen Amphora des Nationalmuseums zu Madrid, auf der der Kampf um Iole zwischen Herakles und Eurytos und dessen Söhnen dargestellt ist. Während der getäuschte Held Rache nimmt, sieht man auch noch die im vorangegangenen Bogenwettkampf benutzte Zelscheibe: eine kleine Halbkugel, in der vier Pfeile stecken ${ }^{14}$. Wie der Sieger hierbei ermittelt wurde, lässt sich nicht zweifelsfrei sagen ${ }^{15}$, was auch für die Deutung eines Zielschießens auf einer rotfigurigen Oinochoe des Museo Nazionale zu Neapel zutrifft. Dargestellt sind drei junge Männer, die mit Pfeil und Bogen einen

11 Hinweise auf Zelschießen bei Pind. Nem. 6, 26 ff.; Theokr. 24,107; Lukian, Herrn. 33. Gewöhnlich führte man die Zielschießen aus dem Stande aus, es konnte aber auch aus dem Laufen und vom Pferd aus geschehen; vgl. E. REISCH, in: RE 1,2 (1894), s. v

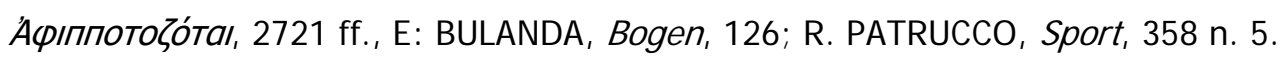

12 Hom. Od. 21, 420 ff.; vgl. F. v. LUSCHAN, Über den antiken Bogen, in: Festschrift für O. Benndorf, 1898, 189 ff.; J. BERARD, Le concours de l'are dans l'Odyssée, in: REG 68,1955, 1 ff.; I. WEI LER, Agon, 239 ff.; P. BRAIN/D. D. SKI NNER, Odysseus and the Axes: Homeric Ballistics Reconstrueted, in: G \& R 25.1, 1978, 55 ff.; R. TÖLLE-KASTENBEIN, Pfeil, $189 \mathrm{ff}$; W. DECKER, Sport, $36 \mathrm{f}$.

13 Hom II. 23, 850 ff.; vgl. Verg. Aen. 5,485 ff. Interessant R. PATRUCCO, Sport, 366: ,..., v'è inoltre una chiara determinazione del bersaglio, concepito con un centro (la colomba) e dei margini (la cordicella), ciò che pone la regolamentazione della gara omerica accanto a quella delle moderne gare di tiro e segno, nelle quali i bersagli sono costituiti di anelli concentrici a cui corrisponde un determinato punteggio, decrescente dal centro ai margini."

${ }^{14}$ Vgl. P. v. BIENKOWSKI, Zwei attische Amphoren in Madrid, in: J ÖAl 3, 1900, 62 ff., Fig. 6; R. TÖLLE-KASTENBEIN, Pfeil, Abb. 12 u. Taf. 1.

${ }^{15}$ P. v. BIENKOWSKI, in: J OEAI 3, 1900, 67: „Während Herakles nach Kreophylos den Vater allein, nach Schol. ad Horn. E 392 nur dessen Söhne im Bogenschießen besiegt, beweist hier die Anzahl der im Zele steckenden Pfeile, dass Herakles wie in der von Apollodor II, 6 und Diodor IV, 31 mitgetheilten Version gleichzeitig mit dem Vater und dessen drei Söhnen zu kämpfen hatte.“ 
hölzernen Hahn beschießen, der auf dem Kapitell einer Säule aufgestellt ist ${ }^{16}$. Da schon drei Pfeile im Hahn bzw. der Säule stecken, und die Bogen gerade neu bespannt werden, muss der einzelne Schütze mehrere, zumindest zwei Versuche gehabt haben. Wir wissen aber nicht, ob die meisten Treffer oder/und das Treffen bestimmter Partien des Tieres für den Sieg den Ausschlag gaben ${ }^{17}$. Die Interpretation R. PATRUCCOS, dass es sich dabei um „una serie di tiri per ogni concorrente, in modo da stabilire, sulla base dei punteggi ottenuti, una classifica di merito ${ }^{\text {“18 }}$ handele, so bestechend sie ist, ist durch die Darstellung nicht zu belegen. Die Zielschießen haben im antiken Bogensport überwogen, und die Genauigkeit des Bogenschusses ist am meisten geschätzt worden. So finden sich zahlreiche Hinweise auf die enorme Präzision der Schüsse. Kaiser Domitian konnte den Kopf eines Tieres mit zwei nacheinander abgeschossenen Pfeilen so treffen, dass sie wie Hörner wirkten, und bewies seine Meisterschaft, indem er durch die ausgebreiteten Finger der rechten Hand eines Jünglings schoss, ohne dabei mit den Pfeilen die Finger zu berühren ${ }^{19}$. Von Commodus erfahren wir, dass er sein Ziel nie verfehlte, wenn er Hunderte von Tieren mit Pfeil und Bogen niederstreckte, so auch Straußen im Lauf den Kopf abschoss ${ }^{20}$, Julius Africanus berichtet von herausragenden Leistungen geübter Bogenschützen wie Mannos, der einem anstürmenden Bären beide Augen ausschoss, und

\footnotetext{
${ }^{16}$ Vgl. H. HEYDEMANN, Die Vasensamm/ungen des Museo Nazionale zu Neapel, Berlin 1872, Nr. 922; L. DEUBNER, Feste, Abb. 20; A. D. TRENDALL, The Red-Figured Vases of Lucania, Campania and Sicily, Oxford 1967, Bd. 2, Taf. 101,3; R. PATRUCCO, Sport, Fig. 179.

${ }^{17}$ Aus Mittelalter und Neuzeit sind in Norddeutschland zahlreiche Schützenschießen (zumeist mit der Armbrust) auf Holzvögel bekannt, bei denen das größte oder schwerste abgeschossene Teil den Sieg brachte und Nebengewinne durch das Abschießen bestimmter Teile wie Flügel, vom Adler gehaltene Insignien etc. erzielt werden konnten; vgl. dazu S. v. PFEIL, Schützenwesen und Schützenfeste in Niedersachsen, Göttingen 1975, 158 u. Abb. 6; G. SPIES, Fünf Jahrhunderte Braunschweiger Schützen, Braunschweig 1970, 6.

${ }^{18}$ Vgl. R. PATRUCCO, Sport, 368; vgl. I. WEILER, Sport, 197.

${ }^{19}$ Suet. Dom. 19.

${ }^{20}$ Herod. 1,15,1 ff.; Cass. Dio 72,18 ff.
} 
von Bardisanes, der wie ein Maler schoss und mit Tausenden von Pfeilen ein Abbild eines starken jungen Mannes zeichnete. Schon unglaublich erscheinen die Leistungen des Syrmos, der die auf inn abgegebenen Pfeile in der Luft abschoss $^{21}$.

Zum Schluss sei noch auf Überlegungen des Julius Africanus hingewiesen. Ihn interessierte die Geschwindigkeit eines Pfeiles. Dazu berichtet er von einem Experiment, dem er persönlich beigewohnt hat: Zehn schussbereite Bogner wurden im Abstand von je einem Plethron (29,60 m) aufgestellt. Sobald der Pfeil des ersten Schützen den zweiten erreichte, sollte dieser schießen, dann der dritte und die anderen ebenso. Julius Africanus kam durch Multiplikation von Strecke und Zeit zu dem Ergebnis, dass der Pfeil in 24 Stunden 20.000 Stadien zurücklegen würde ${ }^{22}$. Kritik an den ungewöhnlichen Berechnungsmethoden mag nach modernem Verständnis berechtigt sein; entscheidend für uns ist jedoch das Interesse der geradezu wissenschaftlichen Methode, die in diesem Experiment zur Bestimmung der Weite zum Ausdruck kommt.

Trotz der dürftigen Quellenlage lässt sich festhalten, dass im antiken Bogensport Weite, Zielgenauigkeit, und selbst Geschwindigkeit Gegenstand von sportlichem Leistungsvergleich und wissenschaftlicher Untersuchung waren.

21 Jul. Afr. Kestoi, Kap. 30 (Hrsg. J. MEURSIUS, Opera omnia, Florenz 1746, 948); vgl. G. A. H. HANSARD, The Book of Archery, London 1940, 438 ff; E. S. MC CARTNEY, Marvellous Feats of Archery, in: CJ 35, 1940, 537 ff; O. v. FLESCHENBERG, Spätantike Anleitung zum Bogenschießen, in: WS 60, 1942, 52; H. KROLL, in: RE 10,1 (1917), s. v. Africanus, 122, meint, dass die Angaben des Jul. Africanus stark nach Jägerlatein klingen. Africanus Kap. 61 ff. Hrsg. J. MEURSIUS, Opera omnia, 961 ff.) beschäftigt sich mit Durchschlagskraft und Schnelligkeit der Pfeile.

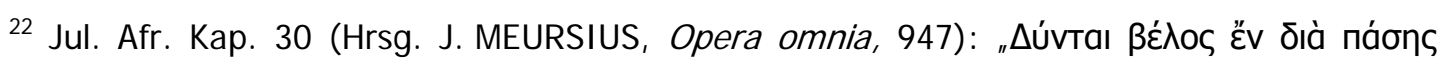

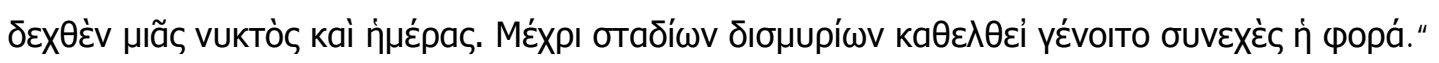


Die Munera unterteilt man in die Kämpfe der Gladiatoren (/udi gladiatori) und in die Tierhetzen ( venationes).

\subsubsection{Gladiatur}

Die Vielzahl der Informationen, die uns über das Gladiatorenwesen ${ }^{2}$ überliefert sind, geben uns ein recht genaues Bild dieses antiken Kampfsports ${ }^{3}$, wobei ein Großteil der Quellen Rückschlüsse auf nahezu alle Aspekte dieses äußerst beliebten Kampfsports ermöglicht ${ }^{4}$.

1 Zu den munera werden neben den Gladiatorenkämpfen und Tierhetzen auch die Naumachien gerechnet. Bei diesen Schaukämpfen zu Schiff - meist von Gladiatoren durchgeführt- waren mehrmals historische Seeschlachten nachempfunden worden. Für unsere Untersuchung sind sie nicht von Bedeutung und können deshalb im Rahmen dieser Arbeit unberücksichtigt bleiben. Vgl. L. FRIEDLÄNDER, SG 2, 92 ff.; E. BERNERT, in: RE 16,2 (1935), s. v. Naumachie, 1970 ff.; G. TRAVERSARI, Spettacoli, 15 ff.; J. P. V. D. BALSDON, Life, 328 f.; A.BERLAN-BAJ ARD, Les spectacles aquatiques romains, Rom 2006 ,bes .33 f.

${ }^{2}$ Vgl. P. J. MEIER, De gladiatura romana, Diss. Bonn 1881; G. LAFAYE, in: DS 2,2 (1898), S. v. Gladiator, 1563 ff.; K. SCHNEIDER, in: RE Suppl. 3 (1918), s. v. Gladiatoren, 760 ff.; L. FRIEDLÄNDER, SG 2, 50 ff.; L. ROBERT, Les gladiateurs dans l'orient grec, Paris 1940 (ND Amsterdam 1971); A. BALIL, La ley gladiatoria de Italica, Madrid 1961; G. VILLE, La gladiature en Occident des origines à la mort de Domitien, Paris/Rom 1981;A.ANASTASI ON, On the Original Meaning of the Gladiatorial Games,in:Nikephoros 23,2010,7-17.

${ }^{3}$ Zur Rechtfertigung der Aufnahme der Gladiatur in den Kanon der antiken Kampfsportarten vgl. D. RAMBA, Gladiatur - Gegenstand sporthistorischer Betrachtung, in: W. BUSS/A. KRÜGER (Hrsg.), Sportgeschichte: Traditionspflege und Wertewandel, Festschrift für W. HENZE, , Duderstadt 1985, 53 ff.

${ }^{4}$ Vgl. G. LAFAYE, in: DS 2.2 (1898), s. v. Gladiator, 1596 ff.; L. ROBERT, Gladiateurs, $293 \mathrm{ff}$. : et passim, A. BALIL, Su gli spettacoli di anfiteatro, in: Mélanges d'archéologie et d'histoire offerts à A. PIGANIOL, Paris 1966, 357 ff., bes. 362 f. 
Die Gladiatoren ${ }^{5}$, von denen uns rund ein Dutzend verschiedener Kampftypen, ${ }^{6}$ die sich durch ihre Ausrüstung unterschieden, ${ }^{7}$ bekannt sind, hatten sich in Schulen ${ }^{8}$ einem speziellen Training ${ }^{9}$ zu unterziehen. In ihren Kämpfen, die zumeist in dafür errichteten Amphitheatern ${ }^{10}$ stattfanden, ließ man überwiegend unterschiedlich ausgerüstete Kämpfer - meist einen leichtund einen schwer bewaffneten Gladiator -gegeneinander antreten ${ }^{11}$. Die

5 Den Gladiatorentruppen gehörten Männer unterschiedlichster Schichten an, vgl. L. FRIEDLÄNDER, SG 2, 54 ff.; M. GRANT, Gladiators, London 1967, 28 ff.; G. VILLE, Gladiature, 227 ff.; K. HOPKINS, Death and Renewal, Cambridge 1983, 23 f. Zu den ,auctorati’ vgl. W. KUNKEL, Auctoratus, in: Eos 48.3, 1957, 207 ff; C. SANFILIPPO, Gli 'Auctorati', in: Studi in onore di A. BISCARDI, Mailand 1982, Bd. 1, 181 ff.; A. GUARINO, I gladiatores e T'auctoramentum, in: Labeo 9, 1983, 19 f. Zur historischen Entwicklung des Gladiatorenwesens vgl. M. GRANT, Gladiators, 58 ff.; W. BACKHAUS, GL 2, 200 f. Zu den Fachbegriffen aus dem Gladiatorenwesen vgl. R. AUGUET, Cruauté et civilisation. Les jeux romains, Paris 1970, 263 ff. Zur Werbung für Gladiatorenschauspiele vgl. J. COLIN, Affiches et invitations pour les spectadles des gladiateurs, in: Pallas 4, 1956, $51 \mathrm{ff}$.

${ }^{6}$ Vgl. G. LAFAYE, in: DS 2.2 (1898), s. v. Gladiator, 1583 ff.; K. SCHNEIDER, in: RE Suppl. 3, (1918), s. v. Gladiatoren, 777 f.; ferner die einschlägigen Artikel in der RE.

7 Detaillierte Beschreibungen verschiedener Gladiatorengattungen bzw. deren besondere Ausrüstung bei S. AURIGEMMA, I mosaici di Zliten, Rom/Mailand 1926; E. LANG, Die Gladiatorendarstellung in der römischen Kunst, Diss. Wien 1937; D. FACCENNA, Rilievi gladiatorii, in: BCAR 76, 1956-58, 37 ff. u. Taf. 1 ff.; L. ROCCHETTI, // mosaico con scene d'arena al Museo Borghese, in: RIA N. S. 10, 1961, 79 ff.; F. COARELLI, // rilievo con scene gladiatorie, in: StudMisc 10, 1967, 85 ff. u. Taf. 33 ff.

8 Vgl. A. M. COLINI/L. COZZA, Ludus magnus, Rom 1962. Zum Betrieb von Gladiatorenschulen und Anmietung von Gladiatorenfamilien vgl. M. CARTER, in: GRBS 44, 2004, 41-68.

${ }^{9}$ Val. Max. 2,3,2. Vgl. G. VILLE, Gladiature, 306; D. RAMBA, Gladiatur, 54.

${ }^{10} \mathrm{Vgl}$. A. NEPPI-MODONA, Gli edifici teatrali greci e romani, Rom 1961; J. PEARSON, Arena. The Story of the Colosseum, New York 1973; R. GRAEFE, Vela erunt, Mainz 1979; A. HÖNLE/A. HENZE, Römische Amphitheater und Stadien, Zürich 1981, 119 ff. Zu Gladiatorenspielen auf dem Forum vgl. G. CARETTONI, Le gallerie ipogee del foro Romano e i ludi gladiatori forensi, in: BCAR 76, 1956-58, 23 ff. u. Taf. 1 ff.

${ }^{11}$ Vgl. zur Kampftaktik einzelner Gladiatorentypen H. WOLLMANN, Retiarier-Darstellungen auf römischen Tonlampen, in: MDAl (R) 32, 1917, 147 ff. 
Kämpfe unterlagen einem Regelwerk ${ }^{12}$ und wurden von einem Kampfrichter geleitet $^{13}$, sogar gelegentlich ein Gefecht von zwei Kampfrichtern ${ }^{14}$.

Erste Erkenntnisse über den sportlichen Werdegang des Kämpfers erhalten wir durch die Angabe seines Ranges; dabei unterschied man zwischen Rekruten (tirones) ${ }^{15}$ und schon öffentlich aufgetretenen Gladiatoren $(\text { veterani })^{16}$, die wiederum in vier Klassen unterteilt waren ${ }^{17}$. Wir wissen aber nicht, wie viele Kämpfe oder Siege nötig waren, um einen Gladiator in den

12 Pseudo-Quint. deci. mai. 9,9; vgl. G. VILLE, Galdiature, 403 ff.

${ }^{13}$ Vgl. L. ROBERT, Gladiatores, 85 f.; G. VILLE, Gladiature, 367 ff; D. RAMBA, Gladiatur, 55.

14 Vgl. J. H. OLIVER, Symmachi. Homo Felix, in: MAAR 25, 1957, 8 ff, bes. 15; A. LA REGINA, Monumento funebre di un triumviro augustale al Museo di Chianti, in: StudMisc 10, 1967, $39 \mathrm{ff}$.

${ }^{15}$ ClL 6, 10197 =ILS 5089: eine armatura Thraecum universa hatte einem tiro aus eigenen Reihen ein Grab gesetzt; CIL 6, 10194=ILS 5088: M. Antonius Exochus, der bei Triumphspielen als tir(o) seinen ersten öffentlichen Kampf bestreitet und im Verlauf des mehrtägigen Festes weitere Auftritte hat; CIL 6, 10236=P. SABBATINI TUMOLESI, Gladiatorum Paria, Rom 1980, Nr. 71: „M(arcus) Attilus, t(iro) v(icit) Hilarius Ner(onianus) (pugnarum) XIV (victoriarum) XIII missus)“; CIL 6,631=ILS 5084 wird tir(o) im Gegensatz zu vet(eranus) gebraucht; CIL 9, 465, 466=ILS 5083, 5083a steht bei einem Teil der Gladiatoren tir(o), wo bei anderen Zahlenangaben stehen. L. ROBERT, Gladiateurs, Nr. 54:

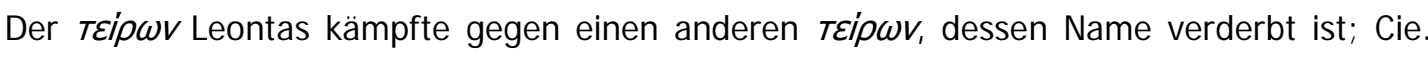
Sest. 36,78 spricht von gladiatores novitios, Th. MOMMSEN, Die Gladiatorentesseren, in: Hermes 21, 1886, 267: „... wie tiro den Fechter bezeichnet, der noch nicht öffentlich aufgetreten ist, so ist veteranus jeder Gladiator, der überhaupt in der Arena gekämpft hat.“ So auch G. LAFAYE, in: DS 2.2 (1898), s. v. Gladiator, 1590; G. VILLE, Gladiature, 311.

$16 \mathrm{CIL}$ 6, 10177=ILS 5104: mirmillonis veterani, ClL 6, 10167=ILS 5125: eq(ues) vet(eranus); CIL 6, 10169; 10176; 10195; vgl. G. VILLE, Gladiature, 312, n. 198.

${ }^{17}$ ClL 5, 5933=ILS 5115: Primo palo, vgl. ClL 6, 10189=ILS 5114; CIL 10, 1926=ILS 5100;

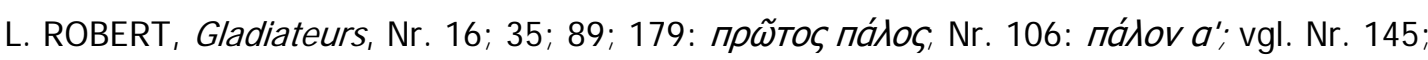

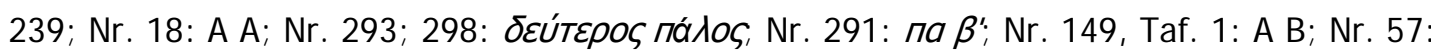

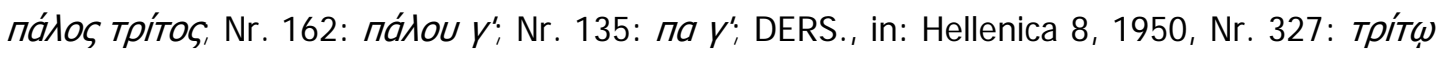
пád $\omega v$, DERS.; Gladiateurs, Nr. 162: пáłou $\delta$; Nr. 148, Taf. 2: A A; vgl. auch DERS., in: RA 31, 1929, 40 f.; DERS., Gladiateurs, $28 \mathrm{f}$. 
ersten Rang oder einen der führenden Ränge aufrücken zu lassen ${ }^{18}$. Zumindest waren die Gladiatoren stolz darauf, einer der ersten Klassen anzugehören. Wir finden Hinweise hierauf häufig in Inschriften und Cassius

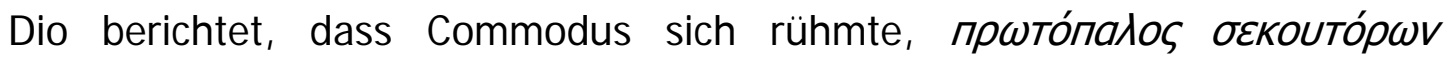
gewesen zu sein ${ }^{19}$.

In dem 176/7 n. Chr. von Marc Aurel und Commodus veranlassten Senatsconsultum zur Kostenminderung der munera wird entsprechend der Gesamtkosten zwischen gregarii und drei bis fünf Klassen besserer Kämpfer unterschieden $^{20}$. Da der Rang des Gladiators in unmittelbarer Beziehung zu seinen Leistungen stand, können wir davon ausgehen, dass bei den Zuschauern wohlbekannte und beliebte Kämpfer auf eine Anzahl von Kämpfen bzw. Siegen verweisen konnten und zu den höherklassigen Rängen zu rechnen $\operatorname{sind}^{21}$. Auf ein zahlreiches und erfolgreiches Auftreten lassen

18 Vgl. L. ROBERT, Gladiateurs, Nr. 16: 1. Rang bei 20 Kämpfen; Nr. 146: 1. Rang bei 12 Kränzen; Nr. 18: 1. Rang bei 8 Kränzen; Nr. 89: 1. Rang bei 8 Kämpfen und 7 Kränzen; Nr. 149: 2. Rang bei 6 Kränzen und 1 Palmzweig; Nr. 298: 2. Rang bei 13 Kämpfen; Nr. $293: 2$. Rang bei 9 Kämpfen; Nr. 135: 3. Rang bei 11 Siegen; DERS., in: Hellenica 8, 1950, Nr. 327: 3. Rang bei 2 Kämpfen; DERS., Gladiateurs, Nr. 148: 4. Rang bei 6 Kränzen und 1 Palmenzweig.

${ }^{19}$ Cass. Dio 72,22,3; vgl. L. ROBERT, Gladiateurs, $28 \mathrm{ff}$.

20 Vgl. J. H. OLIVER/R. E. A. PALMER, Minutes of an Act of the Roman Senate, in: Hesperia 24/4, 1955, 320 ff.; ClL 4, 8590: der Verkauf zweier erstklassiger (primae rationis) Gladiatoren, vgl. P. SABBATINI TUMOLESI, Gladiatorum, 26.

${ }^{21} \mathrm{ClL} 4$, 4289= ILS 5142b: puellarum decus; Cl L 4, 4342=I LS 5142a: suspirium puellarum. Über den Stargladiator Pacideianus vgl. Lucil. 4,149 ff.; über den Stargladiator Heimes vgl. Man. 5,24. Suet. Caes. 26: "Gladiatores notos, sieubi infestis spectatoribus dimicarent, vi rapiendos reservandos mandabat". Vgl. auch CIL 4, 1179; 9975; P. SABBATINI TUMOLESI, Gladiatorum, Nr. 10 u. 52; 22; J. M. C. TOYNBEE, Art in Roman Britain, London 1962, 190; Sen. ep. 7,4: Postulaticii; vgl. dazu R. ISIDORI FRASCA, Ludi, 95. 


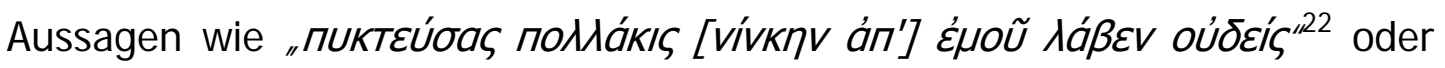

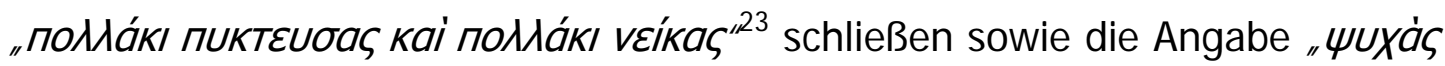

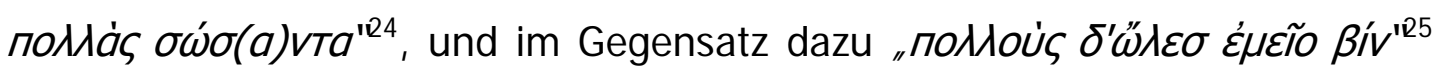
und „, über Zahl der Kämpfe bzw. Siege sehr deutlich die Bedingungen dieses harten Kampfsportes aufzeigen ${ }^{27}$. Nun haben wir im Epitaph des Gladiators

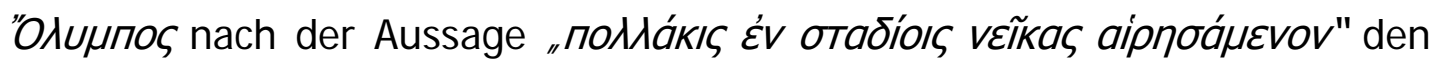

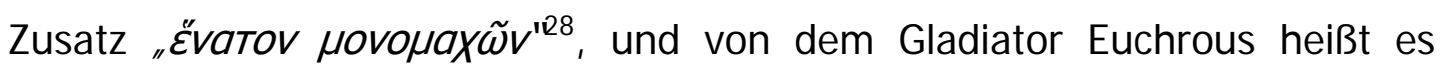

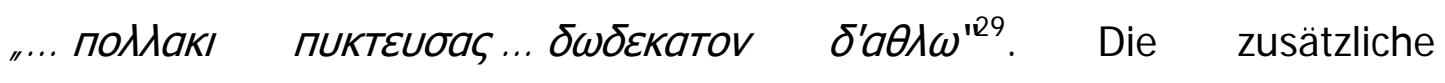
Zahlenangabe lässt Rückschlüsse auf den Umfang der Leistungen zu, die in den angeführten Inschriften erwähnt werden, was sich auch mit den konkreten Angaben einer Vielzahl von Inschriften decken würde und durch die Tatsache bestärkt wird, dass die meisten Gladiatoren nur einmal pro J ahr kämpften ${ }^{30}$. Bei der Angabe einer Zahl ohne nähere Bestimmung lässt es sich nicht zweifelsfrei entscheiden, ob damit die Zahl der Kämpfe oder der Siege gemeint ist. Von der Mehrzahl der Gelehrten wird die Zahl als Angabe der

${ }^{22}$ Vgl. E. LOVATELLI CAETANI, Di due rilievi gladiatorii, in: MDAI (R) 15, 1900, 105 ff., Fig.

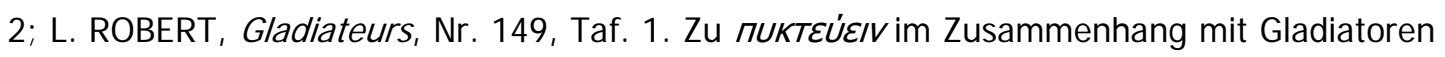
vgl. L. ROBERT, in: RA 31, 2, 1929, 24 ff.; DERS., Gladiateurs, 16 ff.

${ }^{23}$ Vgl. L. ROBERT, in: Hellenica 5, 1948, Nr. 314.

24 .Vgl.L. ROBERT, Gladiateurs, Nr. 55.

${ }^{25}$ Vgl.L. ROBERT, Gladiateurs, Nr. 214, Taf. 3.

${ }^{26}$ Vgl.L. ROBERT, Gladiateurs, Nr. 84.

27 So lässt auch der Dialog des Gladiators Symmachus der Mosaikinschrift CIL 6, 10205=ILS 5140b auf eine regelmäßige Tötung des Unterlegenen schließen. Vgl. J. H. OLIVER, in: MAAR 25, 1957, 12.

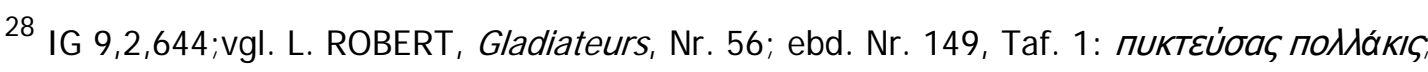
dargestellt sind sechs Lorbeerkränze und ein Palmwedel.

${ }^{29}$ Vgl. J. ROGER, in: RA 1945, 51 ff.; L. ROBERT, in: Hellenica 5, 1948, Nr. 314.

30 Vgl. A. ElTER, Die Gladiatorentesseren, Rh. M. 41, 1886, 531; L. ROBERT, Gladiateurs, 294; J. P. V. D. BALSDON, Life, 301; D. MATZ, Charioteers and Gladiatore: Some Comparison Based on Epigraphical Evidence, in: CB 56, 1980, 37; vgl. Cass. Dio 73,22,3, zu der maßlosen Übertreibung des Commodus. 
Kämpfe verstanden ${ }^{31}$. Dieser Zahl konnte ein Zusatz über den Ausgang des letzten (dargestellten) Kampfes beigefügt $\operatorname{sein}^{32}$; die dafür verwandten Abkürzungen waren V(icit), $M$ (issus), $P$ (eriit), (Obiit) und $\Theta(a v \omega ́ v)^{33}$. Eindeutig bestimmen lässt sich die Zahlenangabe, wenn ihr

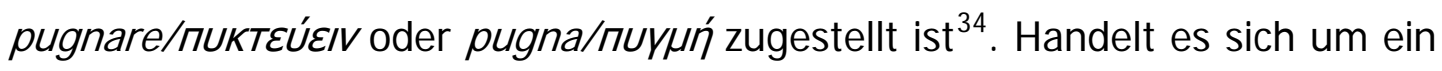
Epitaph, ist der todbringende Kampf meist nicht in die Gesamtzahl der Gefechte aufgenommen ${ }^{35}$ und nur gelegentlich ausdrücklich erwähnt ${ }^{36}$. Der Angabe der Kämpfe konnte der Zusatz folgen, dass man mit dem Gegner

${ }^{31}$ Vgl. A. MAU, Iscrizioni gladiatorie di Pompei, in: MDAI (R) 15, 1890, 32; DERS., Pompeji, 226; E. LOVATELLI CAETANI, Di un frammento marmoreo con rilievi gladiatorii, in: BCAR 23, 1895, 257 n. 1: Cosi nelle iscrizioni ove comparisce un sol numero è sempre quello de' combattimenti; P. SABBATINI TUMOLESI, Gladiatorum, Nr. 29; 32; V. KOCKEL, Die Grabbauten vor dem Herkulaner Tor in Pompeji, Göttingen 1983, 80 ff.; A. GARCÍA Y BELLIDO, Lapidas funerarias de gladiadores de Hispania, in: AEA 33, 1960, Nr. 1; 2; 3; 4; 13, ergänzt nicht wie die oben genannten 'pugnarum', sondern 'pugnavit'. Die höchsten Angaben in diesem Bereich finden wir in einer pompejanischen Inschrift (CIL 4, 2451), die vielleicht eine Auflistung besonders bekannter Gladiatoren ist, in der vier Kämpfer 75, ein Gladiator 100 und ein anderer sogar 150 Gefechte absolviert haben muss. G. VILLE, Gladiature, 315 n. 204, glaubt, dass die Zahlen die Siege angeben. Eine andere hohe Zahl von 107 Kämpfen in Cl L 4, 1422.

$32 \mathrm{ClL} 4$, 1182; vgl. J. OVERBECKIA. MAU, Pompeji in seinen Gebäuden, Alterthümern und Kunstwerken, Leipzig 1884, 188 ff., Fig. 108 f.; F. COARELLI, in: StudMisc 10, 1965, 97, Taf. 42 ff.; P. SABBATINI TUMOLESI, Gladiatorum, Nr. 29, Taf. 3; V. KOCKEL, Grabbauten, 80 ff.,

33 Vgl. G. LAFAYE, in: DS 2,2 (1898), s. v. Gladiator, 1597; A. HÖNLE/A. HENZE, Amphitheater, 24 f.; G. R. WATSON, Theta Nigrum, in: J RS 52, 1952, 56 ff.

${ }^{34} \mathrm{CIL}$ 6, 10195=ILS 5090: pugnavit XVIII; CIL 5, 3465=ILS 5117: pugnarum XXVII; vgl. G.

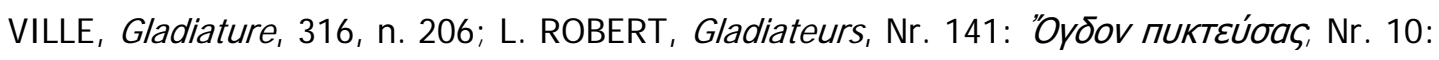

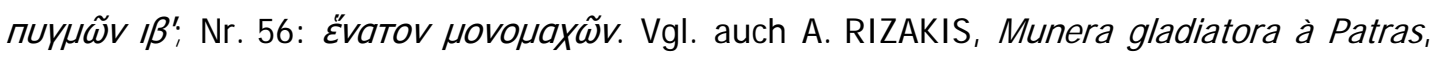
in: BCH 108, 1984, 537 u. Fig. 3.

${ }^{35}$ Vgl. G. VI LLE, Gladiature, 311 n. 196. Meist überlebten die meisten Beteiligten ein munuS, vgl. L. ROBERT, Gladiateurs, 267 ff.; A. HÖNLE/A. HENZE, Amphitheater, 210, n. 54.

36 CIL 5, 3468=ILS 5122: Pugnar(um) X hic XI deceptus; CIL 5, 3466=ILS 5121:

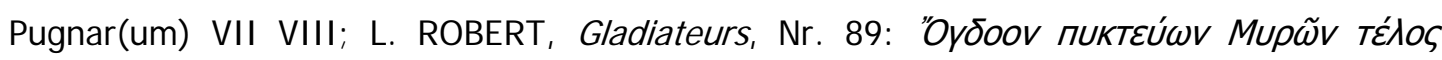
हैoXov. 
zusammen ums Leben gekommen ${ }^{37}$ oder von Verbrechern getötet worden war $^{38}$, zumeist ganz allgemein gefasst, dass das Schicksal den Tod herbeigeführt habe ${ }^{39}$. Besonders wurde hervorgehoben, wenn der Gladiator unbesiegt geblieben war $^{40}$. Auch konnte der Angabe der Kämpfe die der Siege beigefügt sein ${ }^{41}$, sowie zuweilen die Zahl der Kämpfe der der Siege entsprochen haben muss, wenn man die der Inschrift zugeordnete Darstellung von Palmzweigen und Kränzen, deren Zahl identisch ist mit der der angegebenen Kämpfe, als Attribute des Sieges versteht ${ }^{42}$.

Am häufigsten finden wir Angaben über den Sieg. Zumeist war eine

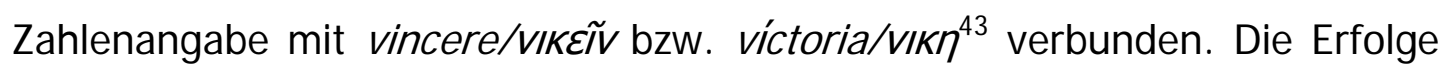
konnten auch durch palma oder corona/orépavos ausgedrückt werden, wobei für letzteres auch das Abkürzungszeichen gebräuchlich war ${ }^{44}$. Auch die

37 CIL 5, 563=ILS 5123: „Decorato retiario, qui peremit Caeruleum et peremptus decidit; ambos extincxit rudis, utrosque protegit rogus. Decoratus scutor, pugnarum XII“.

$38 \mathrm{ClL}$ 5, 3468=ILS 5112: pug(narum) V deceptus a latronebus.

${ }^{39} \mathrm{Cl}$ 5, 3466=ILS 5111: pug(narum) XIII fato deceptus non ab homine.

${ }^{40} \mathrm{CIL} 5,3465=$ ILS 5117: retiario invictos pugnarum XXVII; vgl. CIL 11, 1070=ILS 5118;

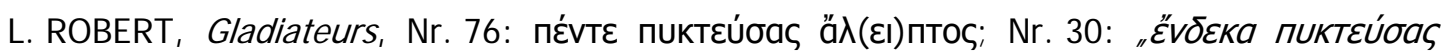

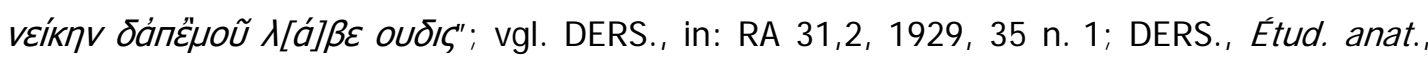
$121 \mathrm{f}$.

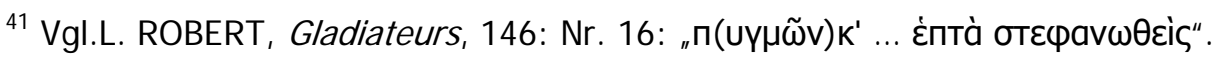

${ }^{42}$ Vgl.L. ROBERT, Gladiateurs, Nr. 68: $\Pi u(y \mu \tilde{\omega} v) \theta^{\prime}$ - dargestellt sind 9 Palmzweige; Nr. 293:

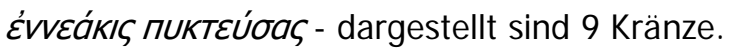

${ }^{43}$ Die Angabe vicit stand gewöhnlich in Aufzählungen der Kampfentscheidungen, vgl. Anm. 61; L. ROBERT, Gladiateurs, Nr. 146: $\triangle \omega ́ \delta \varepsilon \kappa a ~ v \varepsilon ı \kappa \eta ́ \sigma a \varsigma, ~ N r . ~ 65: ~ s i e b e n ~ M a l ~ v \varepsilon ı \kappa \tilde{\omega ; ~ N r . ~ 52 ; ~}$ 53: évíka. Cl L 13, 5354: vic(toriarum) II; zu víkn vgl. L. ROBERT, Gladiateurs, 302 f. Ebd.

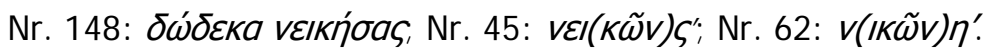

44 ClL 2, 1739=ILS 5098: Palmaru(m) XX; A. GARCíA Y BELLIDO, in: AEA 33, 1960, Nr. 5:

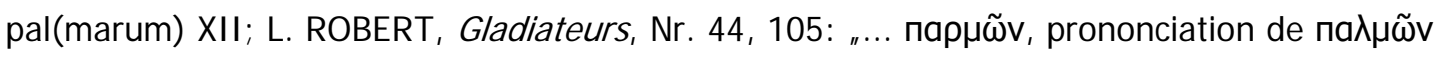
et transcription du latin palmarum $\mathrm{X}$, ne me paraît pas non plus justifiable; nous n'en avons aucun exemple“.; CIL 12, 3332=ILS 5087: cor(onarum) trium; CIL 12, 5907: coronar(um) XX; zu $\sigma \varepsilon(\varphi a ́ v \omega v)$ in Ergebnislisten vgl. L. ROBERT, Gladiateurs, 52; DERS.; Nr. 124:

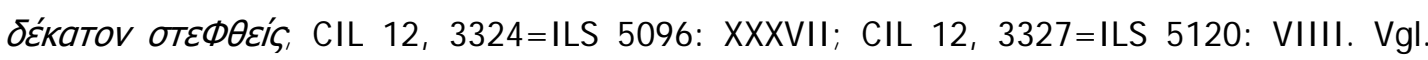
L. ROBERT, Gladiateurs, Nr. 180. 
bildliche Darstellung der Siegesattribute konnte auf den Sieg oder die Anzahl der Siege verweisen. Die palma wurde symbolisch neben die Inschrift oder den siegreichen Kämpfer gesetzt ${ }^{45}$, zumeist wurde der Palmwedel, oft von stattlicher Größe, vom Gladiator im Arm gehalten ${ }^{46}$. Bei der Darstellung der Kränze, die in ihrem Ausmaß bescheidener ausfielen, waren meist mehrere beigesetzt ${ }^{47}$. Ebenfalls konnten beide Attribute des Sieges vom Gladiator in linker und rechter Hand gehalten werden ${ }^{48}$. Häufig findet man auch die Darstellung eines meist großen Palmzweiges und zahlreicher $\mathrm{Kränze}^{49}$. Inschriftliche Angaben und dargestellte Siegesattribute konnten sich gegenseitig bestätigen ${ }^{50}$, aber ebenso deutlich voneinander abweichen ${ }^{51}$.

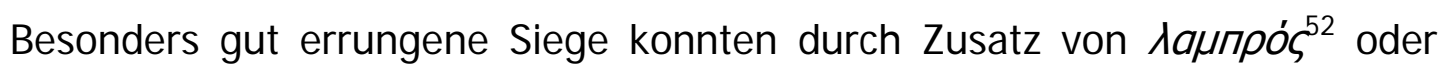
Bändern an palma oder corona ${ }^{53}$ kenntlich gemacht werden.

Ein weiterer Grad der Differenzierung des Sieges bestand darin, die Siege zu unterteilen in solche, die mit einem Palmenzweig honoriert wurden, und solche, die mit einem Kranz ausgezeichnet wurden, wobei letzteres als ehrenvoller galt. Diese Unterteilung ist in griechischen Inschriften zweifelsfrei

45 Vgl. F. CUMONT, Gladiateurs et acteurs dans Le Pont, in: Festschrift fur O. HIRSCHFELD, 1903, 273; L. ROBERT, Gladiateurs, Nr. 123; DERS., in: Hellenica 5, 1948, 98, Taf. 12.

${ }^{46}$ Vgl. E. PFUHL/H. MÖBIUS, Die ostgriechischen Grabreliefs, Mainz 1979, Textband 2: Nr. 1226 f., $1230 \mathrm{ff}, 1242,1234,1242$ f., 1263; Tafelband 2, Tafeln $184 \mathrm{ff}$.

47 Ebd. Nr. 1196, Taf. 181; Nr. 1197, Taf. 181; Nr. 1222, Taf. 184.

${ }^{48}$ Vgl. L. ROBERT, Gladiateurs, Nr. 34.

49 Ebd. Nr. 148, Taf. 2; Nr. 149, Taf. 1; E. PFUHL/H. MÖBIUS, Grabreliefs, Nr. 1196 f. u. Taf. 181.

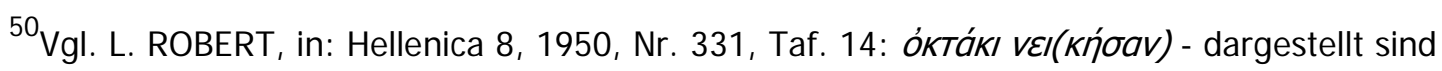

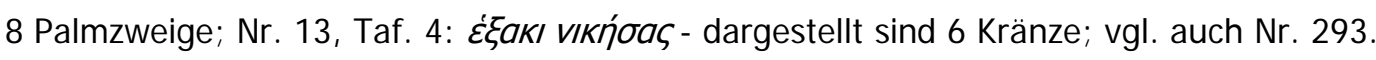

${ }^{51}$ Vgl. L. ROBERT, Gladiateurs, Nr. 52, wo bei neuen Siegen elf Kränze dargestellt sind.

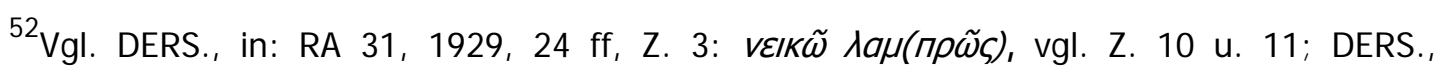

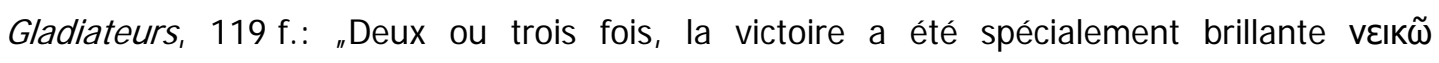
$\lambda a \mu(п р \tilde{\omega} \varsigma)$; peut-être y a-t-il là une distinction correspondant à celle entre les victoires et les couronnes".

53 Ebd. Nr. 301, Taf. 20; vgl. Nr. 25. 
nachzuweisen ${ }^{54}$. In lateinischen Inschriften finden wir zwei Zahlenangaben, die durch ein $C,<,>$ oder / voneinander getrennt sind, wobei in fast allen Fällen die erste Ziffer größer oder gleich der zweiten ist ${ }^{55}$. In Analogie zu den griechischen Inschriften glauben einige Gelehrte, die Ziffern gäben palmae und coronae $\mathrm{an}^{56}$, andere sehen darin die Angabe der Kämpfe und Siege ${ }^{57}$. Beiden Formen konnte eine Angabe über den Ausgang des letzten (dargestellten) Kampfes beigefügt sein ${ }^{58}$.

Wir finden aber auch andere Formen der Differenzierung des Kampfausganges. Wie wir schon oben gesehen hatten, konnte ein Kampf

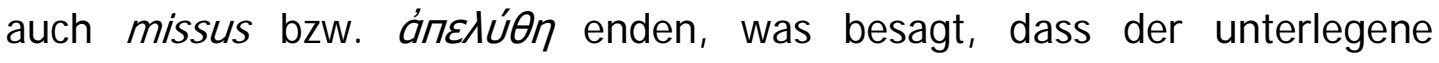
Gladiator, da er gut gekämpft hatte, auf Geheiß des Spielgebers bzw. des

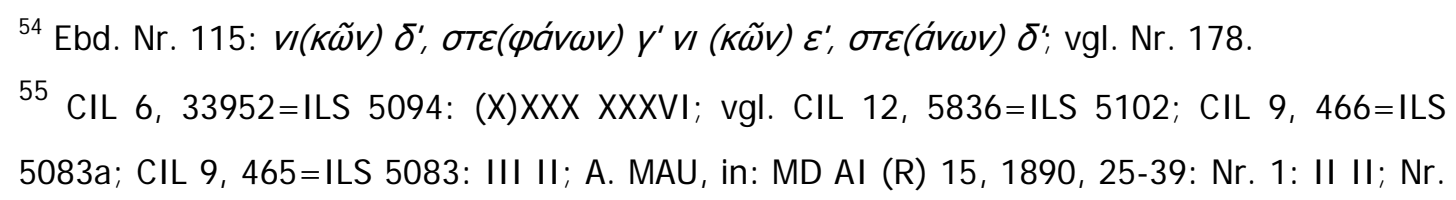
7: XIIII XI; Nr. 9: III III; Nr. 14: III III.

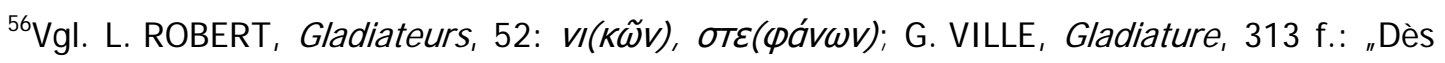
lors, les choses sont claires: le premier chiffre désigne les victoires, le second, les couronnes - apparemment des victoires plus brillantes que les autres et qui ont valu au gladiateur, outre la palme, cette récompense supplémentaire; nous développerons donc ainsi les textes latins: (victoriarum) tot, (coronarum) tot." A. GARCÍA Y BELLIDO, in : AEA 33, 1960, 10: p(almarum) I C(oronarum) I; anders G. VILLE, Gladiature, 315 n. 202.

57 A. MAU, in: MDAl (R) 15, 1890, 35 f.: „Perciò mi pare metodo sano lasciar da parte le iscrizioni greche, riflettendo che nell'oriente potevano esservi, ed evidentemente vi erano, altri costumi, e attenerci a quanto risulta dai titoli latini, che cioè nelle iscrizioni di Venosa e di Venafro il primo numero è quello dei combattimenti, il secondo quello delle vittorie." Aber E. CAETTANI-LOVATELLI, in: MDAI (R) 15, 1900, 102: "Comunque si sia è questa una difficoltà, che non siamo ancora in grado di spianare."

${ }^{58}$ CIL 4, 10236a: M Attilus V(icit) L Raecius XU XII M(issus). Vgl. CIL 4, 10237; 10238; IG

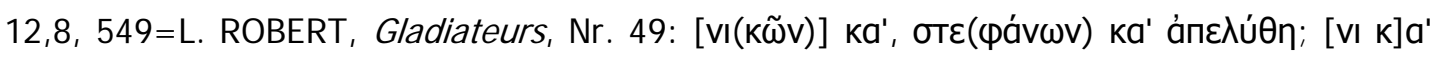

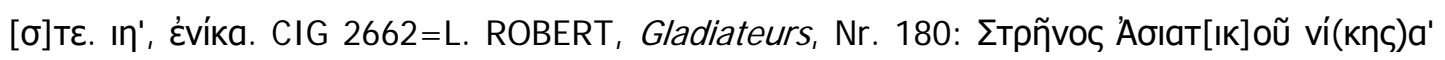

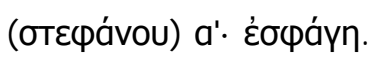


Publikums geschont und entlassen wurde ${ }^{59}$. Angesehener als missus war der Kampfausgang stans missus bzw. orás, was einem Unentschieden gleichkam $^{60}$. Zeichnete sich in einem engagiert geführten Kampf aufgrund der Gleichheit der Kontrahenten keine Entscheidung ab, so wurde der Kampf gestoppt und die Kämpfer als stantes missi entlassen. So konnte ein Rechenschaftsbericht eines Gladiators die Zahl der Kämpfe, der Siege, der Unentschieden und der verlorenen Kämpfe, die aber mit einer Begnadigung endeten, aufweisen ${ }^{61}$. Auch sind gelegentlich die Etappen einer Gladiatorenlaufbahn chronologisch geordnet mit Angabe des Veranstaltungsortes, dem Namen des Gegners und der Kampfentscheidung überliefert ${ }^{62}$.

59 ClL 10, 7297=ILS 5113 (mis(sus) IUI ; CIL 6, 10194=ILS 5088: Miss(um) fe(cit) (?); ClL 9, 1671=ILS 5134: Missos! missos!; vgl. C. WEICKERT, Gladiatoren-Relief der Münchner Glyptothek, Münchner Jahrbuch der bildenden Kunst N. F. Bd. 2, 1925, Heft 1/2, 24 f. Zu

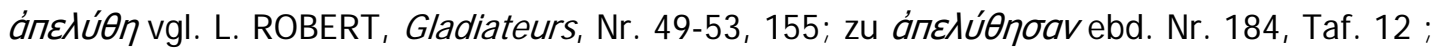
vgl. G. VILLE, Gladiature, $410 \mathrm{ff}$.

60 ClL 12, 2747=ILS 5133: stantes-missi; vgl. CIL 6, 10194=ILS 5088; CIL 6, 33983=ILS 5106; ClL 10, 7297=ILS 5113; CIL 15, 6244a=5135. Sen. epist. 92,25; Mart. de spect. 29; P.-J. MEIER, Gladiatura, 49: Indicatur igitur verbis 'stans mitti' incertum genus pugnae, ubi neuter vincit aut vincitur. Vgl. G. LAFAYE, in: DS 2.2 (1898), s. v. Gladiator, 1595 f.; G. VILLE, Gladiature, 421-424; L. ROBERT, in: RA 31,2, 1929, 27: „కTác placé entre les deux

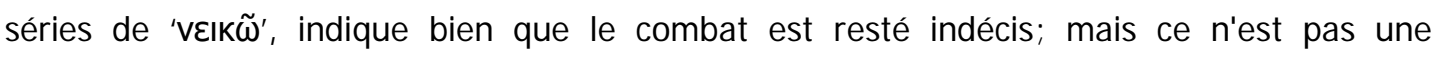
abréviation; c'est le participe de iotavaí; la langue technique de la gladiature en rend compte: orác traduit le latin 'stans'."; vgl. DERS., Gladiateurs, Nr. 65.

61 CIL 10, 7297=ILS 5113; pugnat XXXIIII, vicit XXI, stans VIIII, mis IIII; vgl. CIL 6, 10189=ILS 5114; CIL 6m 33983=ILS 5106. Zur Zeichensprache des Publikums bezüglich der Entscheidungüber Tötung oder Entlassung des Unterlegenen vgl. A. CORBEILL, Thumb in Ancient Rome: Pollex as Index, in: MAAR 42, 1997, 1ff.

${ }^{62} \mathrm{CIL}$ 6, 33988: "III Felicem; IV eundem Felicem; V Ursulu(m); XIIII, Victorinu(m)"; vgl. E. LOVATELLI CAETANI, Di un frammento marmoreo con rilievi gladiatorii, in: BCAR 23, 1895, 253-263, Taf. 14=Scritti vari, Rom 1898, 64-79, Fig. 1; ClL 6, 33980=ILS 5139: „Improbum ... XI Pantheriscum XII Hilarum XI”. Vgl. E. LOVATELLI CAETANI, in: BCAR 23, 263-279, Taf. 15=Scritti vari 79 ff., Fig. 2 f.; CIL 6, 10194=ILS 5088: „Rom. ob triump. divi 
Trainai die II tir. cum Araxe Caes. st. miss.; Rom. mun. eiusd. die VIIII Fimbriam lib. VIII miss. fe.; Rom. mun. eiusd".Vgl. hierzu W. DECKER, Sport am Nil, Hildesheim 2012, 135 f. 


\subsubsection{Venatio}

Eine Untersuchung der sportlichen Leistungen der Tierkämpfer (venatores, bestiarii, кuvnYor), worunter man die im Rahmen eines munus auftretenden Kämpfer zu verstehen hat - zu unterscheiden von den in freier Wildbahn jagenden venatores -, hebt sich doch erheblich von den zuvor behandelten Gladiatoren ab. Trotz mancher Gemeinsamkeit - so wurden auch die venatores in Schulen ausgebildet ${ }^{1}$ und kannten den Zusammenschluss in familiae $^{2}$, wie auch ein gemeinsames Auftreten von Tierkämpfern und Gladiatoren bei Veranstaltungen, in Vor- und Nachmittagsprogramm getrennt, üblich war $^{3}$ - ist aufgrund der vielfältigen Darbietungsweisen der venatores ${ }^{4}$ eine komplexere Betrachtungsweise geboten, die jedoch durch das spärliche Quellenmaterial erheblich erschwert wird.

Die Tierkämpfer unterschieden sich untereinander vor allem durch die Art ihrer Bewaffnung, was auch z. T. durch die Tiere bedingt war, gegen die sie antraten. Am weitaus häufigsten fand hierbei der Jagdspieß (venabulum)

${ }^{1} \mathrm{ClL}$ 14, 2922=ILS 1420 und CIL 8, 7039=ILS 1437: /udus matutinus, L. ROBERT, in:

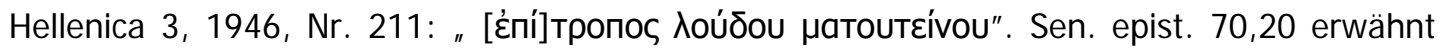
einen /udus bestiariorum.

2 CIL 5, 2541: familia venatoria; vgl. G. VILLE, Gladiature, 346; J. W. SALOMONSON, in: BABesch 35, 1960, 50 ff.; L. FOUCHER, in: OMRL 45, 1964, 87; M. A. BESCHAOUCH, in: CRAI 1966, 134 ff.; K. M. D. DUNBABI N, The Mosaics of Roman North Africa, Oxford 1978, 79 ff. Vgl. auch L. ROBERT, Gladiateurs, Nr. 61: venatores setzen Bronzestatue ihrem medicus. Zu venationes der iuvenes vgl. G. PFISTER, Die Erneuerung der römischen iuventus durch Augustus, Diss. Regensburg 1977, 59 f.; DIES., GL 2, 265.

3 Vgl. L. ROBERT, Gladiateurs, 309; P. SABBATINI TUMOLESI, Gladiatorum, $139 \mathrm{ff} ; \mathrm{zu}$ munus iustum oder legitimum vgl. G. VILLE, Gladiature, 155.

4 R. AUGUET, Cruauté, 108: „.... les techniques de combat étaient extrêmement nombreuses." R. DELBRÜCK, Consulardiptychen, 75 ff., unterscheidet zwischen eigentlichen Tierkämpfern und Tierspielern. Zu den Bestrebungen der Spielgeber, abwechslungsreiche

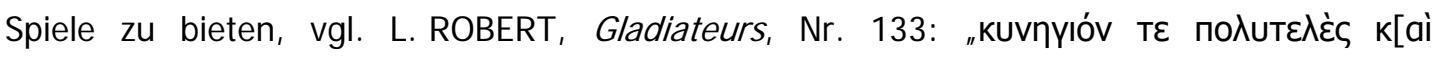
пáp]aঠołov". 
Verwendung ${ }^{5}$; andere benutzte Waffen waren Schwert ${ }^{6}$, Dolch ${ }^{7}$, Stock ${ }^{8}$, Lasso $^{9}$, Peitsche ${ }^{10}$, Pfeil und Bogen ${ }^{11}$ oder die mit Boxhandschuhen bewehrten Fäuste ${ }^{12}$. Zum Blenden oder Reizen der Tiere gebrauchte man gelegentlich eine mappa ${ }^{13}$. In den thessalischen Stierspielen, bei denen der Tierkämpfer zu Pferde den Stier jagte, sich dann auf das ermüdete Tier stürzte und es an den Hörnern zu Boden riss, gelegentlich auch durch Genickbrechen tötete, halfen nur Kraft und Geschicklichkeit ${ }^{14}$. Die Tötung der

\footnotetext{
${ }^{5}$ Vgl. J. E. CAPPS, in: Hesperia (Suppl.) 8, 1949, 69: „... spear the favorite weapon for slaying wild beasts both in venationes and in actual hunts." L. ROBERT, Gladiateurs, 324:

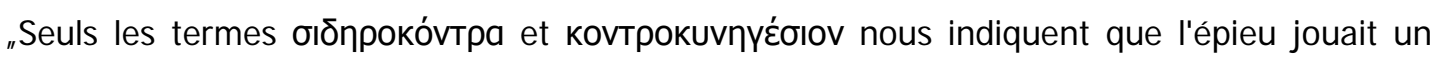
rôle essentiel."
}

${ }^{6}$ Vgl. A. HÖNLE/A. HENZE, Amphitheater, Fig. 63; R. AUGUET, Cruauté, 106.

${ }^{7}$ Vgl. L. ROBERT, Gladiateurs, Nr. 27, pl. 24; Nr. 114; 233.

${ }^{8}$ Ebd. Nr. 219, pl. 25; Nr. 230.

${ }^{9}$ Vgl. R. DELBRÜCK, Consulardiptychen, Nr. 9; Nr. 11; Nr. 21; Nr. 57; K. M. D. DUNBABIN, Mosaics, $75 \mathrm{f}$.

10 Vgl. K. PARLASCA, Die römischen Mosaiken in Deutschland, Berlin 1959, Taf. 37,3; A. HÖNLE/A. HENZE, Amphitheater, Fig. 40c; R. DELBRÜCK, Consulardiptychen, Nr. 21; L. ROBERT, Gladiateurs, Nr. 114; 12; Nr. 264; DERS., in: Hellenica 8, 1950, Nr. 329; R. AUGUET, Cruauté, 112.

${ }^{11}$ Cass. Dio 72,18,1.

12 Vgl. Ph. BRUNEAU, in: BCH 86, 1962, Fig. 19; R. AUGUET, Cruauté, 101; A. HÖNLE/A. HENZE, Amphitheater, Fig. 74; A. GOCEVA, in: Altertum 27, 2, 1981, Fig. 1. L. ROBERT, Gladiateurs, Nr. 27, pl. 24: mit nackten Fäusten gegen einen Bären. Vgl. Plin. nat. 8,130. J. M. BLAZQUEZ, in: Zephyrus 13, 1962, Fig. 6 f.

${ }^{13}$ Vgl. L. ROBERT, Gladiateurs, Nr. 72; 114; 132; 264. Sen de ira 3,30: ursos leonesque mappa proritat. Zu unterschiedlich gerüsteten venatores vgl. L. FOUCHER, in: OMRL 45, 1964, 101 ff, Fig. 7 u. Taf. 11.

14 Suet. Claud, 21,3; Plin. nat. 8,182. Vgl. O. LIERMANN, Annalecta, 27 ff; L. ROBERT, Gladiateurs, 315 ff.; B. MAIURI, in: RAL ser. 8, Vol. 2, 1947 503; J. AYMARD, in: LEC 23, 1955, 261; J. P. V. D. BALSDON, Life, 307. J. M. BLAZQUEZ, in: Zephyrus 13, 1962, Fig. 8. Cassiodor var. 5,42 lobt die Gewandtheit und Schnelligkeit der Tierkämpfer. Vgl. zu Mut, Geschick und Technik der Tierkämpfer L. ROBERT, Gladiateurs, Nr. 81; DERS., in: Hellenica 5, 1948, Nr. 318. 
innen entgegengestellten Tiere war die Hauptaufgabe der Tierkämpfer. Dennoch wissen wir durch zahlreiche Darstellungen, dass die venatores ihren Mut und ihr Geschick im Kampf mit den Tieren auf vielfältige Weise demonstrieren konnten. So finden wir Drehverschläge (cochleae) ${ }^{15}$ und Klettergerüste ${ }^{16}$ in den Arenen, die den unbewaffneten venatores die Chance gaben, sich dem Angriff der Tiere zu entziehen. Einen bedeutenden Platz bei den Spielen nahmen die von den venatores ausgeführten Sprünge mit $^{17}$ und ohne ${ }^{18}$ Stab über zumeist anstürmende Bären, Raubkatzen und Stiere ein. Diese Darbietungen konnten für sich stehen ${ }^{19}$ oder dem todbringenden

15 Vgl. J. SABATIER, Médailles Contorniates, Paris 1860, pl. 4 f., 9; E. SAGLIO, in: DS 1,2 (1887), s. v. cochlea, 1265 f. u. Fig. 1686f.; R. DELBRÜCK, Consulardiptychen, Nr. 9; 12; A. ALFÖLDI, Die Kontorniat-Medaillons, Berlin 1976, Nr. 207; Taf. 173,12; 174,1.2; Nr. 208; Taf. 26,10.

16 Vgl. L. DEUBNER, in: MDAl (A) 27, 1902, 259 f. und Abb. 3; R. DELBRÜCK, Consulardiptychen, Nr. 12; vgl. L. ROBERT, Gladiateurs, $327 \mathrm{f}$.

17 Vgl. J. SABATIER, Contorniates, pl. 8; G. LAFAYE, in: DS 1.2 (1887), s. v. contomonobolon, 1485; H. B. WALTERS, Catalogue of Greek and Roman Lamps in the British Museum, London 1914, Nr. 1189 u. Fig. 252; T. L. LEAR, in: AJA 30, 1926, 453, Fig. 7; R. DELBRÜCK, Consulardiptychen, Nr. 12; L. ROBERT, Gladiateurs, Nr. 60 u. 326 f.; DERS., in: Hellenica 8, 1950, Nr. 334; A. ALFÖLDI, Kontorniat-Medaillons, Nr. 211, Taf 174,3.4. Vgl. auch Anth. Pal. 9,533.

18 Vgl. L. DEUBER, in: MDAl (A) 27, 1902, 260 u. Abb. 5; L. ROBERT, Gladiateurs, Nr. 27; DERS., in: Hellenica 8, 1950, Nr. 340 u. 60, n. 1 u. 2.

19 Auf einem Mosaik aus El-Djem kämpfen mehrere Männer mit nackten Händen oder nur mit Seil und Lasso gerüstet gegen mehrere Tiere, und es hat den Anschein, dass die Tiere nicht getötet, sondern nur niedergeworfen und eventuell in einer anderen venatio Verwendung finden sollten; vgl. J. W. SALOMONSON, in: BABesch 35, 1960, 37 ff. u. Fig. 8 f.; K. M. D. DUNBABIN, Mosaics, 71 u. Fig. 56. G. JENNISON, Animais for Show and Pleasure in Ancient Rome, Manchester 1937, 179, meint, dass im 4. u. 5. Jh. n. Chr. die venationes entschärft wurden und die Zuschauer mehr an den Tricks und der Geschicklichkeit der Darsteller als am Tod der Tiere interessiert waren. Bei Martial 13,98 und 13,100 fordert das Publikum die missio des Tieres. 
Gefecht vorangehen ${ }^{20}$. Das Erlegen der Tiere galt als besonders ruhmvoll, wenn der Todesstoß nicht nur elegant, sondern auch wohl gezielt gesetzt war. ${ }^{21}$ So war es vorbildhaft, die Tiere durch einen Stoß in den Hals bzw. in die Brust zu töten ${ }^{22}$. Ebenso zeichnete sich ein Tierkämpfer aus, wenn ihm der tödliche Stoß beim ersten Versuch gelang ${ }^{23}$.

Was Mut und Geschicklichkeit anbelangt, so wird man beim Aufeinandertreffen von venator und Tier einen Kampf gegen Raubkatzen,

${ }^{20}$ Vgl. R. DELBRÜCK, Consulardiptychen, Nr. 57: Ein venator fängt mit dem J agdspieß einen anspringenden Bären ab, der schon blutet und ein Lasso um den Hals hat. Zu апотоноৎ bei venationes vgl. L. ROBERT, Gladiateurs, Nr. 139. Sen. de ira 3,43,2 berichtet von einem Kampf zweier Tiere mit anschließendem Gefecht des siegreichen Tieres mit einem Tierkämpfer.

${ }^{21}$ Vgl. G. LAFAYE, in: DS 5 (1924), s. v. venatio, 704; L. ROBERT, Gladiateurs, 326; J. AYMARD, in: LEC 23, 1955, 264; A. HÖNLE/A. HENZE, Amphitheater, 66.

22 Vgl. R. DELBRÜCK, Consulardiptychen, Nr. 9; L. ROBERT, Gladiateurs, Nr. 27; K. PARLASCA, Mosaiken, Taf. 90, 2.4; L. ROCCHETTI, in: RIA N. S. 10, 1961, Fig. 1, 3, 6, 8, 12 , 14; A. HÖNLE/A. HENZE, Amphitheater, Fig. 9, 33b, 42b, 55. Vgl. Opp. hal. 2,354 ff. Gelegentlich hat der Spieß das Tier durchdrungen: J. OVERBECK/A. MAU, Pompeji, Fig. 114; V. KOCKEL, Grabbauten, Taf. 32,2; L. ROCCHETTI, in: RIA NS., 10, 1961, Fig. 6; A. HÖNLE/A. HENZE, Amphitheater, Fig. 21; 33b. Bei L. ROBERT, in: Hellenica 7, 1949, Nr. 321, wird ein Spieß benutzt, der hinter der Spitze einen Querbalken hat, damit der Spieß nicht zu tief in das Tier eindringt, schnell wieder herausgezogen und zum nächsten Angriff verwendet werden kann. Misslungene Versuche: K. PARLASCA, Mosaiken, Taf. 39,1; R. AUGUET, Cruauté, 114 (in den Leib der Raubkatze); A. HÖNLE/A. HENZE, Amphitheater, Fig. 34; 40b; J. M. C. TOYNBEE, Tierwelt der Antike, Mainz 1983, Fig. 27 (Stöße auf den Schädel der Raubkatze).

${ }^{23}$ Vgl. J. AYMARD, in: LEC 23, 1955, 265 f. A. AUDOLLENT, Defixiorum tabellae, Paris 1904, Nr. 247: „obliga Gallicu quen peperit Prima ut neque ursu neque tauru singulis plagis occidat neque binis plagis occidat neque ternis plagis occidat tauru ursu ...; SHA Gallien duo 12, 13 ff: nam cum taurum ingentem in harenam misisset exissetque ad eum feriendum venator neque productum decies potuisset occidere, coronam venatori misit. mussanctibusque cunctis, quid rei esset, quod homo ineptissimus coronaretur, ille per curionem dici iussit: taurum totiens non ferire difficile est." 
besonders gefährliche Bären oder zuvor gereizte Stiere ${ }^{24}$ höher einschätzen müssen als gegen relativ harmlose einheimische Tiere ${ }^{25}$, wobei insgesamt gesagt werden kann, dass die Gefahr für den Tierkämpfer, getötet zu werden, nicht sehr groß war ${ }^{26}$.

Nun könnte man in Anlehnung an die Gladiatoreninschriften vermuten, dass die Tierkämpfer uns Bericht über die Zahl ihrer Auftritte oder Gefechte, ihrer Siege oder errungenen Kränze ${ }^{27}$ oder die Anzahl (und Spezies) der von innen getöteten Tiere überlieferten, doch leider sind die Zeugnisse in diesem Bereich recht spärlich. Vorausschicken muss man, dass die venatores Allroundkämpfer waren ${ }^{28}$, die gegen vielerlei Arten von Tieren anzutreten hatten, da in den Veranstaltungen häufig unterschiedliche Spezies geboten

24 CL 10, 6012=ILS 5062: ursi crudeles. Mart. de spect. 19 berichtet, dass Stiere durch Puppen aus rotem Stoff gereizt wurden. Taurocentae reizten die Tiere mit Nadeln oder setzten sie mit Fackeln in Brand; vgl. J. AYMARD, in: LEC 23, 1955, 264; J. P. V. D. BALSDON, Life, 313: Die Spielgeber waren bemüht, möglichst gefährliche Tiere in ihren Spielen bieten zu können, vgl. L. ROBERT, in: Hellenica 5, 1948, 90.

25 Mit dem Ausgang der Republik wurden zunehmend ausländische Tiere verwendet; vgl. dazu und zu den Zeiten der Einführung von den Römern unbekannten Tieren F. DREXEL, SG 4, $268 \mathrm{ff} ; \mathrm{G}$. J ENNISON, Animals, $42 \mathrm{ff}$.

${ }^{26}$ G. JENNISON, Animals, 194: „The ,venatores' were always skilled men who ran perhaps no greater risk than a Spanish toréador.“ Dagegen L. ROBERT, Gladiateurs, Nr. 191 a; J. W. SALOMONSON, in: BABesch 35, 1960, 41 f.: zwei bestiarii von Bären getötet. ClL 8, 11914: von Stier getötet. M. I. ROSTOWZEW, Römische Bleitesserae, in: Klio (Beiheft) 5, 1906, 53: Ankündigung erfolgreicher Tiere.

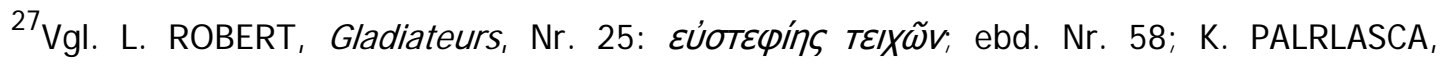
Mosaiken, Taf. 90,1: R. DELBRÜCK, Consulardiptychen, Nr. 12, 20, 37, 58: Siegerkränze und andere Siegerpreise; ebd. Nr. 10: Preisrichter setzt kniendem venator Kranz auf. J. SABATIER, Contorniates, pl. 10,1: eine corona und eine palma. Vgl. J. AYMARD, in: LEC 23, 1955, 265. Zu den Verdienstmöglichkeiten der venatores vgl. M. A. BESCHAOUCH, in: CRAI 1966, 150; K. M. D. DUNBABIN, Mosaics, 68.

28 Mart, de spect. 15; 28;vgl. R. DELBRÜCK, Consulardiptychen, 75; A. HÖNLE, Das Mosaik von Zliten, in: AW 13,4, 1982, 28. 
wurden. ${ }^{29}$ Trotzdem wissen wir von venatores, die auf bestimmte Tiere spezialisiert waren ${ }^{30}$. Meist stand ein venator einem Tier gegenüber, und nur gelegentlich gab es eine Überzahl der einen Seite ${ }^{31}$.

Was die Zahl der gebotenen Tiere anbelangt, so wissen wir von besonderen kaiserlichen Veranstaltungen, bei denen extrem große Mengen geboten wurden, die selbst bei Abstrichen aufgrund von Übertreibungen in den Quellen $^{32}$ noch gewaltig erscheinen. Als herausragende Beispiele mögen die 11.000 Tiere bei den Spielen des Trajan im Jahre $114 \mathrm{n} . \mathrm{Chr}^{33}$ und die zur Einweihung des Amphitheatrum Flavium gestellten 9.000 Tiere angeführt $\operatorname{sein}^{34}$. Die principes versuchten, in Zahl, Ausgefallenheit oder Darbietung zuvor noch nicht gesehener Tiere einander zu übertreffen. Für die venationes aber, die im ganzen Imperium von Beamten oder Priestern nach dem Vorbild

\footnotetext{
${ }^{29}$ Vgl. G. JENNISON, Animals, 42 ff; L. ROBERT, Gladiateurs, Nr. 15: „ठóvta Өnpıouaxías dìa

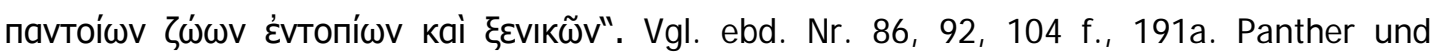
Leoparden waren bei den venationes die häufigsten außereuropäischen Tiere, vgl. L. FRIEDLÄNDER, SG 2, 269. Vgl. CIL 10, 1074=ILS 5053; CIL 10, 6012=ILS 5062; ILS 5063a.

${ }^{30}$ Vgl. L. ROBERT, Gladiateurs,314.

${ }^{31}$ Vgl. L. ROBERT, in: Hellenica 3, 1946, 139: zehn verschiedene Tiere gegen zwei bestiarii. K. PARLASCA, Mosaiken, Taf. 88,3: zwei venatores und neun Tiere. Ebd. helfen zwei Tierkämpfer einem dritten gegen einen Bären. K. M. D. DUNBABIN, Mosaics, Fig. 53; A. HÖNLE/A HENZE, Amphitheater, Fig. 34 u. 89; J. M. C. TOYNBEE, Tierwelt, Fig. 27: venator hilft seinem Kollegen gegen eine Raubkatze.

32 Cass. Dio 43,22,4.

33 Cass. Dio 68,15.

34 Cass. Dio 66,25. Weitere außergewöhnliche Mengen an Tieren: SHA Hadr. 19,3: „Athenis mille ferarum venationem in stadio exhibuit"; Suet. Tit. 7,3: 5.000 wilde Tiere an einem Tage getötet; Res gestae 22: Augustus ließ in 26 venationes 3.500 Tiere töten. Vgl. zu weiteren besonders hohen Zahlen G. VILLE, Gladiature, $398 \mathrm{f}$.
} 
der in Rom durchgeführten Veranstaltungen gegeben wurden, ist in jeder Hinsicht ein viel bescheideneres Maß anzulegen ${ }^{35}$.

Nur von wenigen venatores haben wir detaillierte Kenntnisse. Ausführlich werden wir über die Leistungen des wohl prominentesten Tierkämpfers, nämlich des Kaisers Commodus, unterrichtet. Er tat sich hervor im Töten besonders selten gezeigter Tiere wie Elefant, Nashorn, Nilpferd, Giraffe und Strau $\AA^{36}$ und konnte sich rühmen, 100 Löwen mit Pfeil und Bogen getötet zu haben, wobei nicht ein einziger Pfeil sein Ziel verfehlte; auch soll er insgesamt mehrere Tausend Tiere getötet haben ${ }^{37}$.

Als berühmtester professioneller Tierkämpfer dürfte der von Martial verehrte Carcophorus gelten, der im Laufe einer Veranstaltung 20 Tiere erlegte: „Herculae laudis numeretur gloria: plus est bis denas pariter perdomuisse feras. “38 Doch von den meisten venatores erfahren wir in dieser Hinsicht gar nichts oder nur wenig Konkretes. Ein Epitaph aus Tomis preist einen

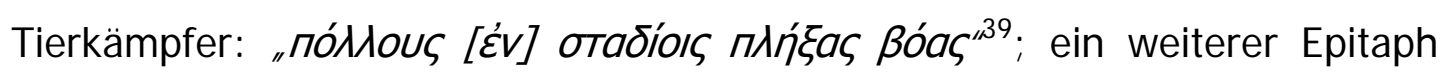

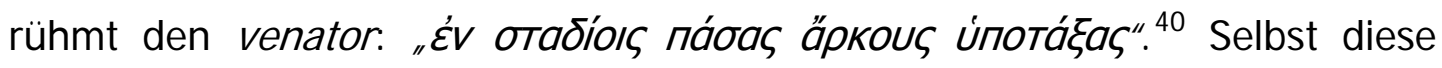
wenig ergiebigen Informationen sind äußerst rar.

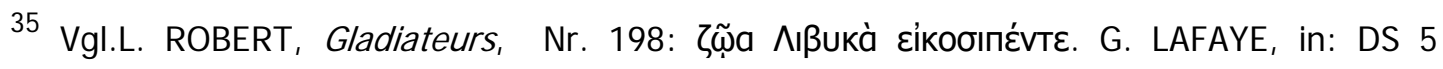
(1914), s. v. venation, 702: „Le nombre des animaux lâches dans l'arène était proportionné à l'importance du munus. "Vgl. G. JENNISON, Animais, 166: "The shows in the amphitheaters in all parts of the Empire were like those of Rome though of a much smaller scale."

${ }^{36}$ Vgl. F. DREXEL, SG 4, 267 ff.; G. JENNISON, Animals, 87.

37 Cass. Dio 73,10,2 f.; 17,2; 18 ff.; Herodian. 1,15,10; Amm. Marc. 31,10, 19; SHA Comm. 13,3 .

38 Mart. de. spect. 15; 28. Vgl. O. WEI NREICH, Studien zu Martial, Stuttgart 1928, $36 \mathrm{ff}$.

${ }^{39}$ Vgl. L. ROBERT, Gladiateurs, Nr. 47.

40 Vgl. F. CUMONT, in: FS O. HIRSCHFELD, 275 f.; E. PFUHU/H. MÖBIUS, Grabreliefs, Nr. 1265, Taf. 188; L. ROBERT, Gladiateurs, Nr. 77; DERS., in: Hellenica 5, 1948, 89. 
Auch bei den munera, insbesondere bei den Gladiatorenspielen, lässt sich festhalten, dass man bestrebt war, Leistung zu messen und ihrer Bedeutung entsprechend zu differenzieren. 
Der Pferdesport der griechisch-römischen Antike bestand aus den hippischen Agonen der Griechen und den circensischen Spielen der Römer.

\subsubsection{Hippische Wettkämpfe der Griechen}

Bei der Abhandlung der hippischen Agone musste von dem Prinzip abgewichen werden, keine Trennung zwischen griechischem und römischem Sport zu machen. Insbesondere das Parteienwesen ${ }^{1}$ gab dem Pferdesport des römischen Circus eine völlig neue Komponente, die eine gesonderte Behandlung erforderte, und bedeutend zu einem Maß differenzierter Darstellung sportlicher Leistungen führte, wie sie wohl für das Altertum einzigartig ist ${ }^{2}$.

Die hippischen Agone bei den griechischen Festspielen ${ }^{3}$ hatten ein äußerst umfangreiches und stark differenziertes Programm ${ }^{4}$. Kannte das olympische Programm - das hinsichtlich der Pferdewettkämpfe für die anderen Festspiele kaum eine Kanon bildende Funktion hatte ${ }^{5}$ - insgesamt acht verschiedene Konkurrenzen ${ }^{6}$, so wissen wir, dass bei den Festspielen zu Athen bis zu 25

\footnotetext{
${ }^{1}$ H. A. HARRIS, SGR, 186, sieht in der wesentlich höheren Zahl der Teilnehmer bei den griechischen Wagenrennen den größten Unterschied zwischen griechischem und römischem Pferdesport.

${ }^{2}$ Ähnliche Verhältnisse finden sich auch im byzantinischen Hippodrom; vgl. A. CAMERON, Porphyrius the Charioteer, Oxford 1973, 66 ff; 71 ff; 206 ff; DERS., Circus Factions, Blues and Greens at Rome and Byzantium, Oxford 1976, $201 \mathrm{ff}$.

${ }^{3}$ Diese erfreuten sich noch bis in das 3. Jh. n. Chr. - trotz wirtschaftlichen Niederganges und der Konkurrenz der circensischen Wagenrennen - zahlreicher Teilnehmer; vgl. L. MORETTI, IAG, 263; J. EBERT, Epigramme, 248.

${ }^{4}$ Eine ausführliche Abhandlung über den antiken Pferdesport ist nach wie vor ein dringliches Desiderat. Nur wenig zu sportlichen Aspekten bietet J. K. ANDERSON, Ancient Greek Horsemanship, Berkeley 1961.

${ }^{5}$ Vgl. I. WEI LER, Sport, 202.

${ }^{6}$ Vgl. L. DREES, Olympia. Götter, Künstler und Athleten, Stuttgart 1967, 168 ff.
} 
unterschiedliche hippische Wettkämpfe ausgetragen wurden ${ }^{7}$. Bei den Pferderennen - Springreiten ${ }^{8}$ und Hindernisrennen ${ }^{9}$ kannte man nicht ${ }^{10}$ - wie für die Wagenrennen unterteilte man die startenden Tiere in Fohlen ( $п \tilde{\omega} \bigwedge o$ ) und erwachsene Pferde $(\tau \varepsilon \dot{\lambda} \mid \varepsilon / o l)^{11}$ und kannte darüber hinaus ein Stutenrennen, bei dem gegen Ende des Rennens der Reiter absprang und neben dem Tier zum Ziel lief ( $\kappa a ́ d n \eta){ }^{12}$.

Neben der Vielzahl der außergewöhnlichen Pferdewettkämpfe, wie etwa Wettreiten auf Kriegspferden ${ }^{13}$, Speerwurf ${ }^{14}$ und Bogenschießwettbewerben vom Pferd ${ }^{15}$, Fackelläufen zu Pferde ${ }^{16}$ und einer Paradedressur ${ }^{17}$ kannte man

7 IG 22, 2313 f.; vgl. L. ZIEHEN, in: RE 18,3 (1949), s. v. Panathenaia, 477 ff J. A. DAVISON, in: JHS 78, 1958, 23 ff; H. A. HARRIS, SGR, 160; I. WEILER, Sport, 205; D. G. KYLE, Athletics, 185 ff; zu der Vielfalt der hippischen Agone bei Festen anderer Städte vgl. R. PATRUCCO, Sport, $384 \mathrm{ff}$.

${ }^{8}$ Das Fehlen des Springreitens ist nicht dadurch zu erklären, dass die Griechen und Römer ohne Steigbügel (und Sattel) ritten; vgl. J. P. V. D. DALSDON, Life, 161; R. PATRUCCO, Sport, 376; I. WEI LER, Sport, 203.

${ }^{9}$ Vgl. M. I. FINLEY/H. W. PLEKET, Spiele, 75.

${ }^{10}$ Der Sprung auf den Rücken des Pferdes mittels Stab dürfte keine eigenständige sportliche Disziplin gewesen sein; vgl. F. WEEGE, in: Jdl 31, 1916, 125 f.; B. SCHRÖDER, Sport, Taf 83a.

11 Vgl. E. N. GARDINER, GASF, 457; B. SCHRÖDER, Sport, 131; F. MEZÖ, Spiele, 139; N. YALOURIS, Games, 122; R. PATRUCCO, Sport, 380; I. WEILER, Sport, 205.

12 Paus. 5,9,1, f.; vgl. E. N. GARDI NER, GASF, 71 f.; K. SCHNEIDER, in: RE 10,2 (1919),

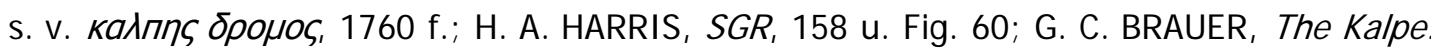
An Agonistic Reference on Several Greek Coins?, in:San 6, 1974-75, 6 f.

13 Syll.3 697; vgl. L. ZEHEN, in: RE 18,3 (1949), s. v. Panathenaia , 478 f.; C. DIEM, Reiterspiel, 21; R. PATRUCCO, Sport, $381 \mathrm{f}$.

14 Syll.3 667; 697; 711; 1055; vgl. A. WILHELM, in: MDAl (A) 30, 1905, 217; E. N. GARDINER, GASF, 354; R. PATRUCCO, Sport, 187 f. u. 381; D. G. KYLE, Athletics, $186 \mathrm{f}$.

${ }^{15}$ Vgl. E. REISCH, in: RE 1,2 (1894), s. v. АӨІппото ६ótaı, 2721 f. 


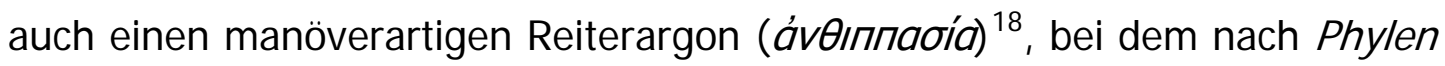
geordnete Abteilungen unter der Leitung eines Hipparchen genau vorgeschriebene Scheinangriffe ritten, deren korrekte Ausführung von einem Kampfgerichtes bewertet wurde ${ }^{19}$.

Die Wagenrennen der Pferde und Fohlen fanden mit dem Zwei- oder Viergespann ${ }^{20}$, die Rennen der Maultiere $(a ́ n \eta ́ v \eta)^{21}$ mit einem zweirädrigen Karren mit Sitzbank statt ${ }^{22}$. Ferner kannte man Wettkämpfe mit Kriegs- ${ }^{23}$ und Prunkwagen ${ }^{24}$ sowie ein Rennen, bei dem sich neben dem Lenker ein mit

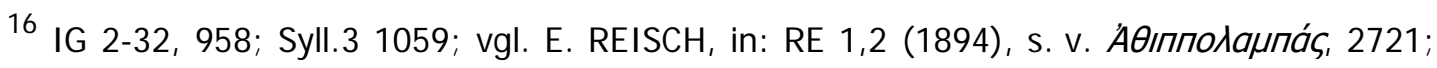
N. YALOURIS, Games, 251; R. PATRUCCO, Sport, 382 u. Fig. 184; D. G. KYLE, Athletics, 193.

${ }^{17}$ Vgl. L. KÖNEN, Eine agonistische Inschrift aus Ägypten und frühptolemäische Königsfeste, Meisenheim 1977,13; G. DUNST, in: ZPE 1, 1967, 239; J. EBERT, in: Stadion 5,1, 1979, 13 u. 19.

18 Xen. hipp. 3,10 ff.; IG 2,2 3130; Syll.3 1074; vgl. E. REISCH, in: RE 1,2 (1894), s. v.

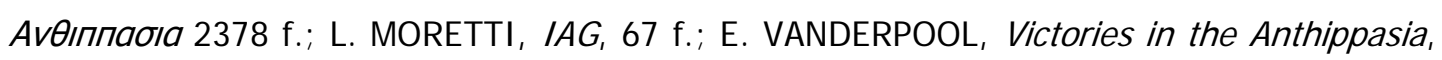
in: Hesperia 43, 1974, 311 ff.; D. G. KYLE, Athletics, 189 f.

19 H. W. PARKE, Festivals of the Athenians, London 1977, 145: „Presumably, then, there were judges who either awarded points for proficiency or deducted them for faults."

${ }^{20}$ Vgl. O. NUOFFER, Der Rennwagen im A/tertum, Diss. Leipzig 1904; E. v. MERKLIN, Der Rennwagen in Griechenland, Diss. Leipzig 1909; K. SCHNEIDER, in: RE 24 (1963), s. v. Quadriga, 681 ff.; H. A. HARRIS, SGR, 171.

${ }^{21}$ Vgl. E. REISCH, in: RE 1,2 (1894), s. v. Ánர́vn, 2695 f.; E. N. GARDINER, GASF, 460; L. MORETTI, Olympionikai, 89; zu Siegern in diesem Wettbewerb vgl. Find. Ol. 5; 6.

${ }^{22}$ Vgl. H. A. HARRIS, SGR, pl. 64 u. 67; N. YALOURIS, Games, Fig. 137; F. BREIN, GL 2, Abb. 40; J. SWADDLING, Games, 71.

23 Syll.3 697; 711; vgl. L. ZIEHEN, in: RE 18,3 (1949), s. v. Panathenaia, 478.

24 IG 2,2, 2311; S11.3 1055; vgl. E. PREUNER, Amphiaraia und Panathenaia, in: Hermes 57, 1922, 90 f.; R. PATRUCCO, Sport, 382; D. G. KYLE, Athletics, 37 u. 186. 
Helm und Schild gerüsteter Agonist befand, der vom Wagen absprang und zum Ziel lief (ánoßárņ) ${ }^{25}$.

Über die griechischen Hippodrome sind wir nur unzureichend informiert ${ }^{26}$. Die genaueste Kenntnis besitzen wir über die Rennbahn von Olympia ${ }^{27}$. Die ausgeklügelte Startanlage ${ }^{28}$ - Pausanias gibt eine genaue Beschreibung ${ }^{29}$ wie auch die Ausmaße der Rennstrecke ${ }^{30}$ dürfen nicht als Maßstab für andere Hippodrome gesehen werden, da sich gewöhnlich Länge und Breite der Rennbahn nach dem zur Verfügung stehenden Raum richteten ${ }^{31}$. Die Rennen erfolgten im Gegenuhrzeigersinn im Allgemeinen um zwei Wendemarken ${ }^{32}$. Die Zahl der zu absolvierenden Runden variierte nach Alter der Pferde bzw. nach Gespannform und bewegte sich in Olympia zwischen einer und zwölf Runden $^{33}$. Die Zahl der an einem Rennen teilnehmenden Pferde bzw. Gespanne richtete sich u. a. nach der Größe des Hippodroms ${ }^{34}$. So konnten

${ }^{25}$ Syll.3 667; 697; 711; 1055; Dion. Hai. 7,73; Paus. 5,9,2; vgl. B. SCHRÖDER, Sport, 135; H. A. THOMPSON, in: AA 1961, 224 ff.; R. PATRUCCO, Sport, 382 ff. u. Fig. 188; J. EBERT, in: Stadion 5,1, 1979, 14; J. SWADDLING, Games, 723; D. G. KYLE, Athletics, 188.

${ }^{26}$ Vgl. L. DREES, Olympia, 112 f.; R. PATRUCCO, Sport, 396; I. WEILER, Sport, 203.

${ }^{27}$ Vgl. A. MALLWITZ, Olympia und seine Bauten, München 1972, 112 f.; J. EBERT, Olympia, $168 \mathrm{f}$.

28 Vgl. Ch. PICARD, Le sculpteur Cleoitas, fils d'Aristocles et les automates de l'aphesis, à I'hippodrome d'Olympie, in: RA 1963, 107 f.; H. A. HARRIS, The Starting-Gate for Chariots at Olympia, in: G\&R 15, 1968, 113 ff.; H. WIEGARTZ, Zur Startanlage im Hippodrom von Olympia, in: Boreas 7, 1984, $41 \mathrm{ff}$.

29 Paus. 6,20,10 ff.

${ }^{30}$ Vgl. L. DREES, Olympia, 114 f.; R. PATRUCCO, Sport, 396; F. BREIN, GL 2, 94.

${ }^{31}$ Vgl. H. A. HARRIS, SGR, 163; N. YALOURIS, Games, 238.

32 In Ausnahmefällen gab es auch Rennen über eine Bahn ohne Wendemal (áкá Syll. ${ }^{3}$ 697; vgl. R. PATRUCCO, Sport, 380.

33 Vgl. L. DREES, Olympia, 14; N. YALOURIS, Games, 239; I. WEILER, Sport, 204; H. WIEGARTZ, in: Boreas 7, 1984, 46.

${ }^{34}$ Vgl. F. MEZÖ, Spiele, 141. 
Pferdehalter, ${ }^{35}$ welche überwiegend nicht selbst ritten bzw. lenkten, ${ }^{36}$ mehrere Pferde bzw. Gespanne ${ }^{37}$ bei einem Rennen starten lassen ${ }^{38}$. Eine Unterteilung betreffs des Alters der Reiter bzw. Lenker kannte man nicht, ${ }^{39}$ und auch das Gewicht der J ockeys spielte hier keine Rolle ${ }^{40}$. Dafür kannte man aber hippische Agone, bei denen die Teilnehmer entsprechend ihres Status $^{41}$ in verschiedene Klassen eingeteilt wurden ${ }^{42}$. Die Vergabe der

35 Neben Individuen treten auch Stadtstaaten als Halter erfolgreicher Pferde bzw. Gespanne auf: vgl. L. MORETTI, Olympionikai, Nr. 39, 207; 233; H. A. HARRIS, SGR, 175 f.; R. PATRUCCO, Sport, 389; H. J. GEHRKE, in: AU 28,5, 1985, 24; J. SWADDLING, Games, 71: "The investment was Propaganda, and good publicity for a state which specialised in horsebreeding."

36 In den 17 Oden des Pindar auf Sieger in hippischen Agonen waren nur zwei Besitzer selber aktiv gewesen (Ol. 6,22 ff.; Isth. 2,22).

37 Nach in: SEG 6, 449, gab es auch Beschränkungen auf ein Pferd bzw. ein Gespann, das ein Pferdehalter pro Wettkampftag starten lassen durfte.

38 Nach Thuk. 6,16,2 hatte Alkibiades bei einem Rennen in Olympia allein sieben Viergespanne starten lassen; vgl. L. MORETTI, Olympionikai, Nr. 345. Auch der Tyrann Dionysius I. von Syrakus hatte bei den Olympischen Spielen 98 (388 v. Chr.) mehrere Viergespanne laufen (Diod. 14,109).

39 Gegen F. MEZÖ, Geschichte, 146, der Paus. 6,2,8 falsch interpretiert; vgl H. BUHMANN, Sieg, 64, n. 2.

40 Paus. 6,13,9 berichtet, dass die Stute des Korinthers Pheidolas, Aura, ihren Reiter schon zu Beginn des Rennes verlor, als erste durchs Ziel ging und den Sieg zugesprochen bekam; vgl. L. MORETTI, Olympionikai, Nr. 147; H. J. GEHRKE, in: AU 28,5,1985, 24. Nach Suet. Nero 24 wurde Nero der Sieg mit dem Viergespann in Olympia zugesprochen, obwohl er aus dem Wagen geschleudert worden war und das Rennen nicht beendet hatte, vgl. J. SWADDLING, Games, 71.

${ }^{41}$ R. PATRUCCO, Sport, 390, stellt hier Vergleiche zu modernen Sportorganisationen an.

42 Syll.3 697; 711; vgl. A. WILHELM, in: MDAl (A) 30, 1905, 217; F. MIE, in: MD Al (A) 34, 1909, 7 ff; C. DIEM, Reiterspiel, 21; L. ZIEHEN, in: RE 18,3 (1949), s. v. Panathenaia, 379 f.; I. WEI LER, Sport, 206. 
Startplätze erfolgte mittels $\operatorname{Los}^{43}$. Die Entscheidung über den Sieg fällte das Schiedsgericht ${ }^{44}$, das seinen Platz auf Höhe der Ziellinie hatte ${ }^{45}$.

Von Siegern hippischer Agone ${ }^{46}$ wurde gelegentlich die Schnelligkeit der Pferde $^{47}$ wie auch die Kunst des Reiters ${ }^{48}$ bzw. des Lenkers ${ }^{49}$ - besonders wenn der Besitzer eigenhändig den Sieg errungen hatte - herausgestellt ${ }^{50}$. Ansonsten finden sich zahlreiche Parallelen zu Aussagen erfolgreicher Leichtund Schwerathleten ${ }^{51}$. Ein Doppelsieg in hippischen Agonen wurde detailliert aufgeführt - wie etwa bei den Siegen des Hellanodiken $\operatorname{Troilos}^{52}$, der mit dem

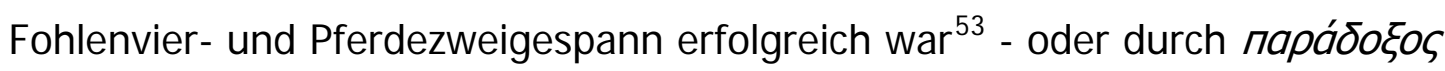

43 Paus. 6,20,10; die Auslosung galt für Wagen- und Pferderennen; vgl. L. DREES, Olympia, 19; H. A. HARRIS, SGR, 155; I. WEILER, Sport, 204. F. MEZÖ, Geschichte, 142, und H. WIEGARTZ, in: Bóreas 7, 1984, 44, streichen heraus, dass es keine Vorläufe gab.

${ }^{44}$ Vgl. H. A. HARRIS, SGR, 180 f.; zur Disqualifikation eines Viergespannes vgl. in: SEG 6, 449; L. ROBERT, in: RA 1978, $284 \mathrm{f}$.

45 Paus. 6,13,9; vgl. R. PATRUCCO, Sport, 401; H. WIEGARTZ, in: Bóreas 7, 1984, 77.

46 Der Pferdesport blieb bis in die Spätzeit Domäne der Aristokraten und Reichen; vgl. E.N. GARDINER, GASF, 463; L. BIZARD, in: BCH 44, 1920, 229 f.; R. PATRUCCO, Sport, 386; F. BREIN, GL 2, 123; I. WEILER, Sport, 200; D. G. KYLE, Athletics, $111 \mathrm{f}$.

${ }^{47}$ Bakchyl. 5,37; IvO 160; vgl. L. BIZARD, in: BCH 44, 1920, 227 ff; L. MORETTI, IAG, Nr. 5, 17; J. EBERT, Epigramme, Nr. 3; 33. Zu Statuen für erfolgreiche Pferde vgl. C. C. VERMEULE, Chariot Groups in Fifth-Century Greek Sculpture, in: JHS 75, 1955, 104 f.; H. BUHMANN, Sieg, $63 \mathrm{ff}$.

48 Ivo 170; vgl. W. W. HYDE, Statues, 53 f.; L. MORETTI, IAG, Nr. 30; J. EBERT, in: WZ Halle 15, 1966, 377 f.; DERS., Epigramme, Nr. 49.

49 Pind. Pyth. 2,5; Isth. 1,15.

50 IG 5, 1, 213; Pind. Isth. 1,15 f.; SEG 11, 1246; L. MORETTI, IAG, Nr. 59; L. SEMMLINGER, Olympia, Nr. 66.

${ }^{51}$ Ein Teil siegreicher Pferdehalter war auch in athletischen Disziplinen erfolgreich.

52 Nach Paus. 6,1,4 führten die Siege des Hellanodiken zu dem Beschluss, die Kampfrichter von der Teilnahme an den Wettbewerben auszuschließen.

53 IvO 166; Paus. 6,1,4;vgl. L. MORETTI, IAG, Nr. 19; DERS., Olympionikai, Nr. 412 f. N. YALOURIS, Games, 238, ordnet fälschlicherweise die Siege getrennt den Olympischen Spielen 102 und $103 \mathrm{zu}$. 
ausgedrückt ${ }^{54}$. Siege in athletischen Disziplinen und zusätzlich in hippischen Agonen wurden besonders hervorgehoben. So konnte Lykinos von Sparta neben dem Waffenlauf auch das Viergespannrennen in Olympia für sich entscheiden ${ }^{55}$, Eubates von Kyrene neben dem Stadionlauf auch das Viergespannrennen dort gewinnen ${ }^{56}$ und Edea siegte bei verschiedenen Festen im Stadionlauf, im Kitharödenwettbewerb und im Pferdewettrennen mit dem Kriegswagen ${ }^{57}$. Rekordverdächtig in diesem Zusammenhang waren die Leistungen des spartanischen Jünglings Enymachratidas ${ }^{58}$, dem es dreimal gelungen war, an ein und demselben Tag drei Laufkonkurrenzen für sich zu entscheiden und zusätlich noch im Pferederennen, das er selbst bestritt, den Sieg davonzutragen ${ }^{59}$.

Gewann man in hippischen Agonen bei verschiedenen Festen, an denen man in Folge teilgenommen hatte ${ }^{60}$, oder bei denselben Spielen mehrmals hintereinander ${ }^{61}$, wurde es besonders vermerkt, wie z. B. bei Hieron I. von Syrakus mit seinem Ausnahmepferd Pherenikos. ${ }^{62}$ So wie besonders bei den Wagenrennen der Ruhm aufeinanderfolgender Siege noch vermehrt wurde,

${ }^{54}$ Vgl. L. MORETTI, IAG, Nr. 89; J. EBERT, in: WZ Halle 15, 1966, 381 ff; DERS. Epigramme, Nr. 81.

55 Paus. 6,2,1; P. Oxy. 222; L. MORETTI, Olympionikai, Nr. 304 u. 324; vgl. A. HÖNLE, Olympia, 154; H. BUHMANN, Sieg, 93.

${ }^{56}$ Aelian. var. hist. 10,2; Jul. Afric. Ol. 93; vgl. L. MORETTI, Olympionikai, Nr. 347 u. 421.

57 Syll. 3. 802; vgl. L. MORETTI, IAG, Nr. 63; vgl. H. A. HARRIS, SGR, 178 f.

58 IG 5,1, 213; L. MORETTI, IAG, Nr. 16.

59 H. A. HARRIS, SGR, 161, wertet diese Erfolge ab, da sie nicht im Rahmen bedeutender Spiele erzielt worden sind.

${ }^{60}$ Hdt. 6, 103; vgl. F. MEZÖ, Geschichte, 146 f.; L. MORETTI, Olympionikai, Nr. 120; 124; 127.

61 Paus. 5,8,11; 6,1,4; 6,13,10; IvO 166;vgl. L. MORETTI, IAG, Nr. 19; J. EBERT, Epigramme, Nr. 38.

62 Pind. Ol. 1; Bakchyl. 5; P. Oxy. 222; vgl. L. MORETTI, Olympionikai, Nr. 221 u. 234. vgl. auch U. v. WI LAMOWITZ, Pindaros, Berlin 1922; 224 ff., zu der Tatsache, dass Kimon I. von Syrakus seine zahlreichen Siege gleich von mehreren bedeutenden Dichtern lobpreisen ließ. 
wenn der Triumph mit denselben Pferden gelungen war, wie es Euagoras aus Sparta ${ }^{63}$ und Kimon aus Athen ${ }^{64}$ bei drei Olympischen Spielen hintereinander geschafft hatten. Besonders hohes Ansehen genossen diejenigen, die unbesiegt geblieben waren ${ }^{65}$. Da es gestattet war, gleichzeitig mehrere Pferde bzw. Gespanne starten zu lassen, war man stolz, neben dem Sieg noch weitere gute Platzierungen ${ }^{66}$ aufweisen zu können. So rühmte sich Alkibiades ${ }^{67}$ bei einem Rennen in Olympia, bei dem er sieben Gespanne starten ließ, was als Rekord herausgestrichen wurde ${ }^{68}$, den ersten, zweiten

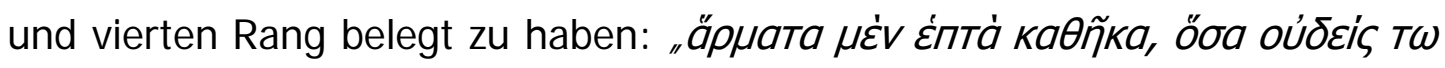

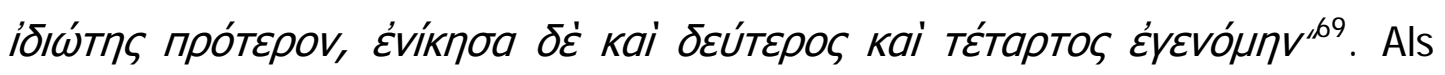
Ausnahme galt der Olympiasieg des Spartaners Lykinos mit dem Fohlenviergespann in der Konkurrenz der ausgewachsenen Pferde ${ }^{70}$.

Wir besitzen auch eine Vielzahl von Siegesverzeichnissen, in denen genauestens die Siege erfasst sind. So konnte man in einem Siegeskatalog mit Angabe des Festortes und der Disziplin die einzelnen Erfolge detailliert auflisten $^{71}$ oder die Siege des Besitzers ${ }^{72}$ oder des Pferdes $^{73}$ mit einer

63 Paus. 6,10,8; vgl. L. MORETTI, Olympionikai, Nr. 110; 113; 117.

64 Hdt. 6, 103; vgl. F. MEZÖ, Geschichte, 144; L. MORETTI, Olympionikai, Nr. 120; 124; 127; H. A. HARRIS, SGR, 175.

${ }^{65}$ Bakchyl. 5,43 ff. (Rennpferd); vgl. L. MORETTI, IAG, Nr. 37; J. EBERT, Epigramme, Nr. 59 (Fohlenviergespann).

${ }^{66}$ Vgl. H. BUHMANN, Sieg, $13 \mathrm{f}$.

${ }^{67}$ Zur politischen Bedeutung der Siege des Alkibiades vgl. A. HÖNLE, O/ympia, 206 ff.; C. W. BOWRA, in: Historia 9, 1960, 68 ff.; D. C. YOUNG, Myth, $100 \mathrm{ff.}$

${ }^{68}$ Vgl. M. N. TOD, in: CQ 53, 1949, 108.

69 Thuk. 6,16,2; Athen. 1,3; Plut. Ale. 11; Dem. 1,1. Euripides (fr. 3 D) und Isokrates $(16,34)$ schreiben ihm den dritten statt des vierten Platzes zu.

70 Paus. 6,2,1; vgl. F. MEZÖ, Geschichte, 145; vgl. L. MORETTI, Olympionikai, Nr. 324; J. SWADDLING, Games, 68.

71 IvO 184; vgl. J. BI NGEN, in: BCH 77, 1953, 630 ff.; L. MORETTI, IAG, Nr. 35; 43.

${ }^{72}$ Vgl. J. EBERT, Epigramme, Nr. 8 (sechs Siege mit dem Viergespann in Nemea). 
konkreten Zahl angeben. Die Zahl der hippischen Siege einer Familie konnte ihren Ausdruck in der Schaffung eines Gesamtdenkmales finden, so wie es sechs Angehörige einer elischen Familie in Olympia errichten ließen ${ }^{74}$. In ähnlicher Weise führten acht Spartaner durch ihre in Reihe aufgestellten Siegerstatuen dem Betrachter eindrucksvoll die Zahl lakonischer Siege mit dem Viergespann in Olympia vor Augen ${ }^{75}$. Wie bei den Leicht- und Schwerathleten konnten auch Sieger hippischer Agone die Summe ihrer Siege in Erfolge in Heiligen Kranzspielen und Wertpreisagonen unterteilen. So führt T. Dominus Prometheus aus Athen ${ }^{76}$, nachdem er seine Siege bei den

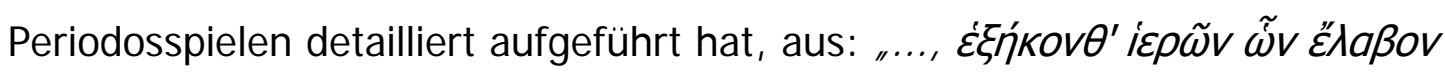

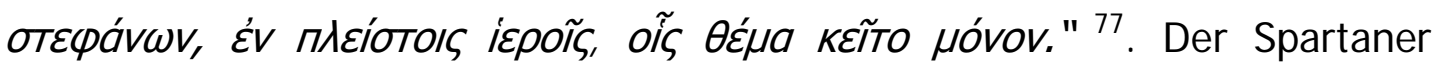
Damonon, der 43 Siege mit dem Viergespann und 18 Siege mit dem Reitpferd, die er alle eigenhändig erzielt hatte, ferner 20 Siege in Laufkonkurrenzen zuzüglich sieben hippischer Erfolge seines Sohnes anführte - allerdings alle bei weniger bedeutenden lokalen Festen ${ }^{78}$ - beanspruchte im Präfix seines enorm langen Siegerepigrammes einen Rekord für sich:

73 Paus. 6,13,9 f.

74 IvO 198-203. Ähnlich die Gruppe von Statuen der Familie des Diagoras von Rhodos; Paus. $6,7,1 \mathrm{f}$.

75 Paus. 6,1,2; 6,2,3; vgl. L. MORETTI, Olympionikai, Nr. 305; 315; 324; 327;339; 373; 381; 386; H. BUHMANN, Sieg, $92 \mathrm{f}$.

${ }^{76}$ H. A. HARRIS, SGR, 175: „It is the sad cry of a man who had bravely tried in the utterly professional world of horse-racing to keep the spirit of amateurism alive. Across the centuries Titus Dominus reaches out a hand to Mr Avery Brundage." Dagegen sieht A. CAMERON, Factions, 205 n. 3 in Titus Domitius „a millionaire pothunter".

${ }^{77}$ Vgl. L. MORETTI, IAG, Nr. 89; DERS., Olympionikai, Nr. 932; J. EBERT, in: WZ Halle 15, 1966, 381 ff; in: SEG 23, 113.

78 H. A. HARRIS, SGR, 161: „In every sport in every age there are those who contrive to shine by carefully avoiding competition with their equals or superiors in skill. Damonon and his son obviously belonged to this not very admirable class." Vgl. J. SWADDLING, Games, 70. 


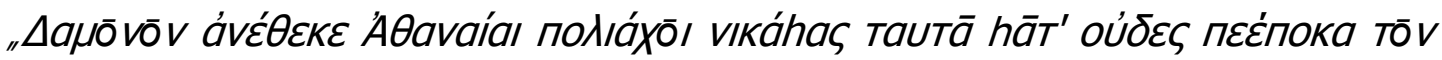
$V \bar{U} V^{.179}$

Erstaunlich ist die geringe Zahl an Periodoniken bei hippischen Agonen ${ }^{80}$. Lediglich die beiden Spartaner Polykles ${ }^{81}$ und Xenarches ${ }^{82}$ sowie der Athener T. Domitius Prometheus ${ }^{83}$ vermochten den Zyklus der Periodosspiele einmal zu gewinnen. Auch die Erst- und/oder Einmaligkeit eines Sieges bei hippischen Agonen wurde oft herausgestrichen. Anaxandros ${ }^{84}$ war der erste Lakedaimonier, der mit dem Viergespann in Olympia gewann, Kleonymos von Rhodos $^{85}$ war der erste, der für seine Heimat den Sieg mit dem Zweigespann bei den Nemeen gewann. Akestorides ${ }^{86}$ siegte als erster der Troer in Olympia mit einem Fohlengespann, und Xenombrotos ${ }^{87}$ gewann als erster Koer mit

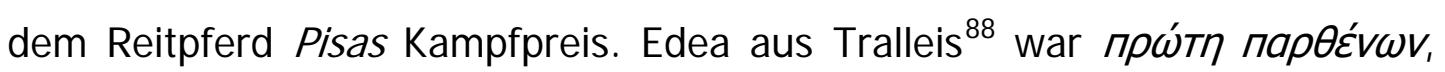
die neben zwei anderen Konkurrenzen mit dem Kriegswagen erfolgreich war $^{89}$, und Kyniska aus Sparta ${ }^{90}$ strich die Einmaligkeit ihres 79 IG 5,1,213; vgl. L. MORETTI, IAG, Nr. 16.

${ }^{80}$ A. CAMERON, Factions, 205; führt die geringe Zahl an Periodoniken in hippischen Agonen auf die hohen Kosten zurück. R. KNAB, Periodoniken, 8, hat die Sieger hippischer Wettkämpfe von seiner Zusammenstellung der Periodoniken mit der Begründung ausgenommen, dass die persönliche Leistung fehle und es kaum anzunehmen sei, dass in allen vier Rennen der Periodos die gleichen Pferde liefen.

81 Paus. 6,1,7;vgl. L. MORETTI, Olympionikai, Nr. 315.

82 Paus. 6,2,1; L. MORETTI, Olympionikai, Nr. 386.

${ }^{83}$ Vgl. L. MORETTI, Olympionikai, Nr. 932.

${ }^{84}$ Paus. 6,1,7; vgl. L. MORETTI, Olympionikai, Nr. 327.

${ }^{85}$ Vgl.J. u. L. ROBERT, in: REG 83,1970, Nr. 417; J. EBERT, Epigramme, Nr. 69.

86 IvO 144; vgl. L. MORETTI, IAG, Nr. 43; DERS., Olympionikai, Nr. 590; J. BINGEN, in: BCH77, 1953, $630 \mathrm{ff}$.

${ }^{87}$ Vgl. W. W. HYDE, Statuis, 53 f.; L. MORETTI, IAG, Nr. 30; DERS., Olympionikai, Nr. 590; J. EBERT, Epigramme, Nr. 49.

88 Syll.3 802; vgl. H. POMTOW, in: Klio 15, 1918, 71 ff.; L. MORETTI, IAG, Nr. 63.

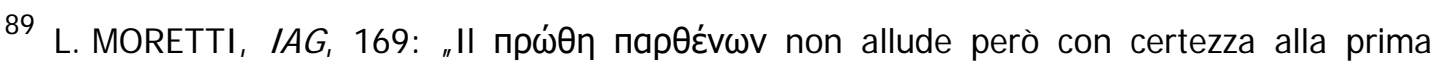
vittoria femminile in senso assoluto: potrebbe darsi che già altre fanciulle avessero vinto in 
Viergespannsieges in Olympia besonders heraus: "Móvav $\delta^{\prime} \dot{\varepsilon} \mu \varepsilon \dot{\varepsilon} \varphi a \mu \prime$

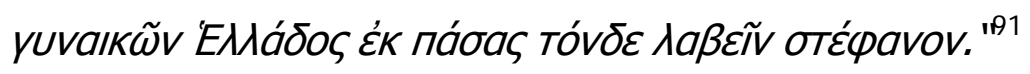

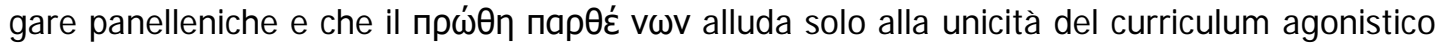
di Trifosa nel suo insieme."

90 Zu Frauen als Rennpferdehalter vgl. F. MEZÖ, Geschichte, $147 \mathrm{f}$.

91 IvO 160; IG 5,1,1564a; vgl. M. N. TOD, in: CQ 53, 1949, 107; L. MORETTI, IAG, Nr. 17; DERS., Olympionikai, Nr. 373; J. EBERT, in: Wz Halle 15, 1966, 393; DERS., Epigramme, Nr. 33, W. DECKER, Nil, 112; Vgl. Anth. Pal. 18,16 u. Paus. 3,8,1; ferner H. BUHMANN, Sieg, 74. 


\subsubsection{Circensische Spiele der Römer}

Als herausragendes Beispiel für umfasssendes Quallenmaterial im antiken Sport kann man die zumeist inschriftlich festgehaltenen Leistungen der Wagenlenker im alten Rom ansehen. ${ }^{1}$ Viele Gemeinsamkeiten von antikem und modernem Rennsport ${ }^{2}$, vor allem in der hohen Wertschätzung der Gewinnsummen ${ }^{3}$, drängen dem Leser geradezu Vergleiche auf. ${ }^{4}$

Die Wagenrennen, deren Ursprung in die Königszeit zurückgeht ${ }^{5}$, gewannen in der Zeit der Republik als fester Bestandteil zahlreicher religiöser Feste ${ }^{6}$ an Bedeutung und waren in der ausgehenden Republik und der Kaiserzeit die alles überragende Sportdisziplin ${ }^{7}$. Die beiden anderen hippischen Konkurrenzen - Rennreiten betrieben die Römer nicht ${ }^{8}$ - waren die Übungen der Desultoren und das Trojaspiel. Bei der ars desultoria ging es vor allem darum, auf dem Pferd Kunststücke zu veranstalten, Gegenstände vom Pferde aus vom Boden zu lesen oder im Galopp von einem auf das andere Pferd zu

\footnotetext{
${ }^{1}$ Vgl. J.-P. THUILLIER, Sport, $113 \mathrm{f}$.
}

${ }^{2}$ Vgl. L. FRIEDLÄNDER, SG 2, 28; H. A. HARRIS, SGR, 194.

${ }^{3}$ Vgl. L. FRIEDLÄNDER, SG 2, 28 f.; A. CAMERON, Porphyrius, 179.

${ }^{4}$ Vgl. H. A. HARRIS, SGR, 194:

${ }^{5}$ Vgl. J. REGNER, in: RE Suppl. 7 (1940), s. v. Ludi circenses, 1626 f.; R. C. BRONSON, Chariot-Racing in Etruria, in: Studi in onore di Luisa Banti, Rom 1965, 89; E. RAWSON, Chariot-Racing in the Roman Republic, in: PBSR 49, 1981, 1-16; J.-P. THUILLIER, Jeux athlétiques, 90 ff. u. 108 f.; J. H. HUMPHREY, Circuses, 64 ff.

${ }^{6}$ Vgl. J. REGNER, in: RE Suppl. 7 (1940), s. v. Ludi circenses, 1650; G. WISSOWA, Religion und Kultus der Römer, München 1971², 449 ff.; I. WEI LER, Sport, 240 ff.

${ }^{7}$ Vgl. L. FRIEDLÄNDER, SG 2, 24; I. WEILER, Sport, 245; zur politischen Bedeutung der Spiele im Prinzipat vgl. T. BOLLINGER, Theatralis Licentia, Winterthur 1969; Z. YAVETZ, Plebs and Princeps, Oxford 1969; zur gezielten Förderung des Spielwesens durch die Kaiser vgl. R. GILBERT, Die Beziehungen zwischen Princeps und stadtrömischer Plebs im frühen Prinzipat, Bochum 1976, bes. $47 \mathrm{ff}$.

${ }^{8}$ Vgl. J. P. V. D. BALSDON, Life, 323; A. CAMERON, Porphyrius, 45; J.-P. THUILLIER, in: Stadion 7.2, 1981, 175; nach Cass. Dio 60,7,3 gab Kaiser Claudius ein Kamelrennen. 
wechseln ${ }^{9}$. Die Sieger dieser oft mit dem Voltigieren verglichenen Wettkämpfe wurden ähnlich den musischen Agonen von einem Kampfgericht bestimmt und nicht bescheiden honoriert ${ }^{10}$. Der Troiae lusus war ein paramilitärisches Formationsreiten, bei dem in Gruppen (turmae) aufgeteilte Reiter - z. B. in Rom die Söhne von Senatoren und Rittern (iuventus) ${ }^{11}$ - nach genau festgelegten Regeln Scheinangriffe gegeneinander ritten ${ }^{12}$. Bei diesen hippischen Agonen, die im ganzen Römischen Reich durchgeführt wurden und sich besonderer Förderung durch Kaiser Augustus erfreuten ${ }^{13}$, wurden die Sieger durch ein Kampfgericht bestimmt ${ }^{14}$. Doch diese beiden Konkurrenzen waren mehr oder weniger nur Pausenfüller für die alles überragenden Wagenrennen ${ }^{15}$.

Die enorme Beliebtheit ${ }^{16}$ der Wagenrennen war in der Endphase ihrer Entwicklung nicht unwesentlich durch das Parteienwesen, das sich im Laufe

${ }^{9}$ Liv. 23,29; 44,9; Suet. Caes. 39; Manil. astron. 5, 85 ff.; vgl. E. POLLACK, in: RE 5 (1903), s. v. Desultor, 255 ff.; J. P. V. D. BALSDON, Life, 323.

10 Vgl. P. VIGNERON, Le cheval dans l'antiquité gréco-romaine, Nancy 1968, $209 \mathrm{ff.;}$ G. PFISTER, GL 2, 255 f.; I. WEILER, Sport, 250; J.-P. THUILLIER, Jeux athlétiques, 97 ff.; A. WACKE, Das Relief-Fragment Nr. 26 aus Mariemont: Zirkus-Szene oder manumissio vindicta?, in: Studi di onore di A. BISCARDI, Bd. 1, Mailand 1982, 116 ff., bes. $138 \mathrm{ff}$.

${ }^{11}$ Vgl. G. PFISTER, Erneuerung , 48 ff.; DIES., GL 2, $250 \mathrm{ff}$.

${ }^{12}$ Vgl. die umfangreiche Auflistung der Quellen bei K. SCHNEIDER, in: RE 13 (1927), s. V. Iusus Troiae, 2059 ff.; vgl. ferner G. GIGLIOLI, in: SfE 3, 1929, 111 ff.; H. v. PETRIKOVITS, in: Klio 32, 1939, 209 ff.; E. MEHL, in: RE Suppl. 8 (1956), s. v. Troiaspiel, 888-905.

${ }^{13}$ Vgl. G. PFISTER; Erneuerung, 3 ff.; I. WEILER, Sport, 226 f.; G. W. HOUSTON, The lusus Troiae and Augustan Patriotism, in: Aug Age 1, 1981-1982, $8 \mathrm{ff}$.

${ }^{14}$ Vgl. K.-W. WEEBER, Troiae lusus. Alter und Entstehung eines Reiterspiels, in: Anc Soc 5, 1974, $171 \mathrm{ff}$.

15 Dion. Hai. 7,73; Suet. Caes. 39; Dio Cass. 51,22.

${ }^{16}$ Auch die Wettleidenschaft steigerte bei manchen das Interesse am Pferdesport; vgl. Juv. 11,201; Mart. 11,1,15; Tert. spect. 16; vgl. J. P. V. D. BALSDON, Life, 318; H. A. HARRIS, $S G R, 223 \mathrm{ff}$. 
der Republik herausgebildet hatte ${ }^{17}$, gefördert worden ${ }^{18}$. Die Aufteilung der Teilnehmer in vier Parteien ${ }^{19}$ - die grüne (factio prasina), die blaue (factio veneta), die rote (factio russata) und die weiße (factio alba bzw. albata) ${ }^{20}$ bewirkte, dass bestimmte soziale Schichten sich mit einer der Parteien identifizierten ${ }^{21}$, was auch einige Kaiser z. T. aus Gunsthascherei taten ${ }^{22}$. Als Vorbild für den Rennbetrieb im gesamten Römischen Imperium kann man die in Rom - besonders im Circus Maximus - abgehaltenen Spiele ansehen ${ }^{23}$, die in Umfang und Prachtentfaltung kaum zu übertreffen gewesen $\operatorname{sind}^{24}$. Als Spielgeber traten die Kaiser bzw. Magistrate auf, die die Rennen starteten, indem sie ein Tuch (mappa) in die Rennbahn warfen ${ }^{25}$. Die Organisation der Spiele oblag den Rennparteien, die einen gewaltigen Stab an Personal unterhielten ${ }^{26}$.

${ }^{17}$ Vgl. A. CAMERON, Factions, $56 \mathrm{ff}$.

18 Vgl. L. FRIEDLÄNDER, SG 2, 37 f.; R. ISIDORI FRASCA, Ludi, 119 ff.; W. BACKHAUS, GL 2, $212 \mathrm{f}$.

${ }^{19}$ Von Domitian waren noch die factio purpurea und die factio aurata hinzugefügt worden, die aber nicht lange selbständig blieben; vgl. Suet. Dom. 7,1; Dio Cass. 67,4,4; ClL 6, 10062. Zum Zusammenschluss der Parteien vgl H. A. HARRIS, SGR, 194; A. CAMERON, Factions, $45 \mathrm{ff}$. u. $69 \mathrm{f}$.

20 Vgl. zu Spekulationen über die Symbolik der Farben der Parteien und anderer Gegebenheiten des Circus M. Floriani Squarciapino, in: RAL 34, 1979, $275 \mathrm{ff}$.

${ }^{21}$ Vgl. A. CAMERON, Factions, 74 ff.; W. BACKHAUS, GL 2, 215.

22 Vgl. L. FRIEDLÄNDER, SG 2, 34 f. und 37 ff.; H. A. HARRIS, SGR, 214 ff.; A. CAMERON, Factions, 157 ff.; W. BACKHAUS, GL 2, 215.

23 Vgl. H. A. HARRIS, SGR, 187; J. H. HUMPHREY, Roman Circuses, London 1986, 540.

${ }^{24}$ Vgl. zu den Kosten der Spiele W. M. GREEN, in: AJPh 51, 1930, 249 f.; M. A. CAVALLARS, in: RhM 122, 1979, 49 ff.

25 Varro ling. 5,153; Mart. 12,29; Suet. Nero 22; vgl. H. GABELMANN, in: AW 11, 1980, 25 ff.; J. H. HUMPHREY, Circuses, 153.

${ }^{26}$ Vgl. L. FRIEDLÄNDER, SG 2, 33 f.; A. NEPPI MODONA, Richerche su alcuni termini relativi ai ludi circensi, in: Hommages à L. Herrmann, in: Coll. Latomus 44, 1960, 562 ff. 
Von den Circusanlagen, die über das ganze Römische Reich verstreut waren, haben wir vor allem durch archäologische Funde recht genaue Kenntnis ${ }^{27}$. Die Größe der Rennbahn war oft durch das Gelände bestimmt ${ }^{28}$. Der Start erfolgte aus Startboxen (carceres) ${ }^{29}$. Wie bei den Wagenrennen griechischer Feste liefen die Gespanne im Gegenuhrzeigersinn um zwei Wendemarken (metae), wobei den römischen Circusanlagen eigen war, dass diese Wendemarken durch einen Wall (spina) verbunden waren ${ }^{30}$. Die Zahl der startenden Gespanne war auf vier, acht und zwölf festgeschrieben ${ }^{31}$. Die Gespannform konnte vom Zwei- bis zum Zehngespann variieren ${ }^{32}$, doch die meisten Rennen wurden mit der Quadriga bestritten ${ }^{33}$. Die Zahl der zu absolvierenden Runden (curricula) betrug gewöhnlich sieben ${ }^{34}$, und die Zahl der im laufenden Rennen beendeten Umläufe wurde den Zuschauern (und Lenkern) durch zwei Anzeigegeräte auf der spina mitgeteilt ${ }^{35}$. Die Zahl der Rennen pro Festtag hatte sich von ursprünglich einer Wettfahrt ${ }^{36}$ beständig erhöht und belief sich seit dem frühen Prinzipat auf gewöhnlich 24

27 Vgl. A. HÖNLE/A HENZE, Amphitheater, 183 ff.; J. H. HUMPHREY, Circuses, $1 \mathrm{ff}$.

${ }^{28}$ Vgl. z. B. M. VICKERS, in: JRS 62, 1972, 25 ff. (Thessaloniki); E. THOMAS, in: Boreas 7 , 1984, 165 (Köln).

29 Vgl. A. BALIL, Mosaicos circenses de Barcelona y Gerona, in: BRAH 151, 1962, 299 ff.; K. M. D. DUNBABIN, Mosaics, 88 ff.; DIES., in: AJA 86, 1982, 78 ff.; J. H. HUMPHREY, Circuses, $132 \mathrm{ff}$.

${ }^{30}$ Vgl. A. BALIL, in: BRAH 151, 1962, 272 ff., bes. 289 ff.; K. M. B. DUNBABIN, Mosaics, 88 ff.; A. HÖNLE/A HENZE, Amphitheater, 93 ff.; J. H. HUMPHREY, Circuses, 175 ff. u. 255 $\mathrm{ff}$.

${ }^{31}$ Vgl. H. A. HARRIS, SGR, 186.

32 Vgl. L. FRIEDLÄNDER, SG 2, 47.

33 Vgl. W. BACKHAUS, GL 2, 214.

${ }^{34}$ Vgl. Suet. Dom. 4, wo die Rundenzahl von 7 auf 5 reduziert wurde, damit die hundert angesetzten Rennen an einem Tag durchgeführt werden konnten.

35 Vgl. W. K. QUINN-SCHOFIELD, in: Latomus 25, 1966, 99 f.; A. BALIL, in: Latomus 25, 1966, 867 ff.; J. H. HUMPHREY, Circuses, 260 ff.

36 Vgl. J. REGNER, in: RE Suppl. 7 (1940), s. v. Ludi circenses, 1632; E. RAWSON, in: PBSR 49, 1981, 15 n. 62. 
Wettrennen pro Veranstaltungstag ${ }^{37}$. Bei Doppelfesten und ganz besonderen Anlässen konnte die Zahl der Rennen noch höher $\operatorname{sein}^{38}$. Die Wagenlenker waren nicht in Altersklassen unterteilt ${ }^{39}$, und ihr Körpergewicht spielte keine Rolle $^{40}$. Spezielle Rennen nach Alter oder Geschlecht der Pferde unterteilt führten die Römer nicht durch ${ }^{41}$. Die Startpositionen bzw. -boxen wurden durch Los ermittelt ${ }^{42}$. Über den Sieg entschied ein Kampfgericht, das auf Höhe der Ziellinie saßs ${ }^{43}$.

Bei der Vielzahl der Angaben, die wir von erfolgreichen Lenkern erhalten haben und die als Grundlage einer Dokumentation ihrer Leistungen dienen, setzen wir mit der Gesamtzahl der bestrittenen Rennen ein ${ }^{44}$. So erfahren wir vom Wagenlenker Crescens die genaue Zahl seiner Starts in seiner knapp

${ }^{37}$ Suet Nero 22; Dio Cass. 59,7; 60,27; vgl. J. P. V. D. BALSDON, Life, 248; A. CAMERON, Porphyrius, 256; A. HÖNLE/A HENZE, Amphitheater, 85 ff.

38 Mart. 8,78,13; Suet. Dom. 4,7.

39 Crescens (ClL 6, 10050=ILS 5285) hatte schon als Dreizehnjähriger an Viergespannrennen teilgenommen. Nach A. CAMERON, Porphyrius, 156, begann eine Wagenlenkerlaufbahn gewöhnlich mit dem sechzehnten Lebensjahr.

40 Plin. nat. 8,160; vgl. J. REGNER, in: RE Suppl. 7 (1940), s. v. Ludi cicenses, 1633; I. WEILER, Sport, 245. Vgl. H. A. HARRIS, SGR, 242 f., zu einem ähnlichen Fall im byzantinischen Circusbetrieb.

${ }^{41}$ Die Pferde liefen gewöhnlich nicht vor dem fünften Lebensjahr (Plin. nat. 8,162; Colum. 6,29,4); vgl. H. A. HARRIS, SGR, 210. Aus den zahlreich überlieferten Pferdenamen lässt sich schließen, dass beim römischen Pferdesport vorwiegend männliche Tiere eingesetzt wurden; vgl. L. FRIEDLÄNDER, SG 2, 31; J. M. C. TOYNBEE, in: PBSR 16, 1948, 24 ff.; DIES., Tierwelt, $164 \mathrm{ff}$.

42 Tert. spect. 16; Symm. or. 9,6; vgl. E. SAGLIO, in: D-S 1 (1887), Fig. 1531; J. P. V. D. BALSDON, Life, 316; A. ALFÖLDI, Kontorniat-Medaillons, Nr. 203; W. BACKHAUS, GL 2, 213; I. WEILER, Sport, 246; J. H. HUMPHREY, Circuses, 154.

43 Plin. nat. 8,65; 35,58; Sen. ep. 108,32; vgl. W. K. QUINN-SCHOFIELD, in: Latomus 25, 1966, 861 ff.; A. BALIL, in: Dar 1, 1973, 371 ff.; J. H. HUMPHREY, Circuses, 595 f.

44 Bei der Zahl der bestrittenen Rennen ist natürlich zu berücksichtigen, dass das vom 1. J h. n. Chr. ständig steigende Angebot an Rennen ganz andere Möglichkeiten eröffnete; vgl. I. WEILER, Sport, 247. 
zehn J ahre währenden Karriere: "missus ostio $D C L X X X V{ }^{A 4}$. Weitaus aktiver war der Lusitaner Diocles, der in den 24 Jahren $^{46}$ seiner überaus erfolgreichen Laufbahn 4.257 Mal an den Start gegangen war: "missus ostio I///CCLVII'A7. Viel häufiger als die Anzahl der Starts erfahren wir die Gesamtzahl der Siege. Eine Ausnahme dabei bildet die Inschrift des Wagenlenkers Scirtus ${ }^{48}$, wo J ahr für J ahr die Anzahl der Siege angegeben ist und am Schluss alle Siege zusammengefasst werden: "Summa summarum quadriga vicit $\mathrm{VII}$ revocatus $/ / /^{\text {'A9 }}$. Die meisten uns überlieferten Wagenlenker waren jedoch wesentlich erfolgreicher. Fuscus ${ }^{50}$ hatte 53 Siege vorzuweisen, ein unbekannter Lenker der Grünen ${ }^{51}$ hatte es mit Vier- und Zweigespann auf 60 Erfolge gebracht, M. Aurelius Mollicius Tatianus ${ }^{52}$ gewann 125 Mal, Musclosus ${ }^{53}$ von der roten Partei hatte 682 Mal gesiegt,

45 ClL 10050=I LS 5285; vgl. E. LOVATELLI CAETANI, La iscrizione di Crescente, in: Antichi monumenti illustrati, Rom 1889, 1 ff.; F. DREXEL, SG 4, 196.

46 Zu Lebensdaten verschiedener Wagenlenker vgl. A. BALIL, in: Mélanges à ANDRÉ PIGANIOL, 362, n. 1. Vgl. auch A. CAMERON, Porphyrius, 3.

${ }^{47}$ CIL 6, 10048=ILS 5287; vgl. F. DREXEL, SG 4, 185 ff.; A. GARCÍA Y BELLIDO, Diodes el 'As' de los circos romanos, in: Nummus 1, 1953, 81 ff.; DERS., El español C. Apuleius Diocles, el más famoso corredor de carros de la antigüedad, in: CAF 14, 1972, 5 ff.

$48 \mathrm{ClL}$ 6, 10051=ILS 5283; vgl. A. E. GORDON, Album of Dated Latin Inscriptions, Berkeley/Los Angeles 1958, Bd. 1, Nr. 60; DERS., Latin Epigraphy, Berkeley/Los Angeles/London 1983, Nr. 37.

49 Eigentümlich ist hier, dass die Siege in einem Wiederholungsrennen von den übrigen Siegen getrennt werden; vgl. zu revocatus bzw. remissus F. DREXEL, SG 4, 182; L. P. WILKINSON, Ovid Recalled, in: JRS 46, 1956, 114 ff.; E. THOMAS, Ovid at the Races. Amores, III, 2; Ars amatoria I, 135-164, in: J. BIBAUW (Hrsg.), Hommages à MARCEL RENARD, Brüssel 1969, Bd. 1, $710 \mathrm{ff}$.

50 ClL 6, 33950; vgl. P. PIERNAVIEJA, Corpus des inscriptiones deportivas de la España romana, Madrid 1977, Nr. 18.

${ }^{51} \mathrm{CIL} 6,10055=$ ILS 5284.

52 CIL 6,10049=ILS 5286; vgl. H. A. HARRIS, SGR, 207.

${ }^{53} \mathrm{CIL} 6,10063=$ I LS 5281. 
Scorpianus ${ }^{54}$ hatte 700 Siege herausfahren können, M. Aurelius Polynices ${ }^{55}$ hatte 739 Palmzweige verliehen bekommen, und ein von Caracalla ermordeter Wagenlenker ${ }^{56}$ war 782 Mal erfolgreich gewesen. Zu den besonders erfolgreichen Lenkern gehörten die miliarii, $d . h$. diejenigen Wagenlenker, die tausend und mehr Siege erzielt hatten ${ }^{57}$. Knapp den Sprung in diesen exklusiven Club hatten Avilius Teres ${ }^{58}$ mit 1.011 und mit 1.025 Siegen ein uns unbekannter Lenker der Grünen geschafft ${ }^{59}$. Zu diesem Kreis der überaus erfolgreichen Lenker gehörte auch P. Aelius Gutta Calpurnianus ${ }^{60}$ mit 1.127, ein weiterer uns unbekannter Lenker der Grünen mit $1.155^{61}$ sowie C. Appuleius Diocles ${ }^{62}$ mit 1.462 und Pontius Epaphroditus ${ }^{63}$ mit 1.467 Siegen. Uns sind sogar Wagenlenker bekannt, die mehrere Tausend Siege erzielt hatten. Flavius Scorpus ${ }^{64}$, der schon mit 27 J ahren starb ${ }^{65}$, hatte 2.048 Erfolge vorzuweisen, M. Aurelius Libero ${ }^{66}$ konnte ${ }^{54}$ ClL 3, 12013; vgl. M. FLORIANI-SQUARCIAPINO, in: RAL 34, 1979, 282.

55 ClL 6, 10049=ILS 5286; vgl. V. SALETTA, Ludi circensi, Rom 1967, 110 ff.; R. ISIDORI FRASCA, Ludi, 124.

${ }^{56}$ Cass.Dio 77,1,2.

57 Vgl. F. DREXEL, SG 4, 180; H. A. HARRIS, SGR, 202; R. PALMIERI, in: RAAN 53, 1978, 60; I. WEILER, Sport, 246.

${ }^{58} \mathrm{ClL}$ 6, 10048=ILS 5287; vgl. L. BORSARI, L'epigrafe onoraria di Avilius Teres agitatore circense, in: BCAR 30, 1902, $177 \mathrm{ff}$.

${ }^{59} \mathrm{Cl}$ 6, 10048=ILS 5287.

${ }^{60} \mathrm{Cl}$ 6, 10047=I LS 5288; vgl. F. DREXEL, SG 4, 179 ff.; A. HÖNLE/A HENZE, Amphitheater, 101.

${ }^{61} \mathrm{ClL} 6,10048=$ ILS 5287.

62 Ebd.

63 Ebd.

64 CIL 6, 10048=ILS 5287.

65 Mart. 10,53,3 über Flavius Scorpus: „I nvida quem Lachesis raptum trieteride nona, dum numerat palmas, credidit esse senem." Vgl. auch R. SYME, Scorpus the Charioteer, in: AJ AH 2, 1977, 86 ff. Zum Tod eines anderen Lenkers vgl. Cl L 8,16566.

66 CIL 6, 10058=ILS 5296; vgl. zur Laufbahn des Libero A. CAMERON, Factions, 11; R. PALMIERI, in: RAAN 53, 1978, 59. 
3.000 Siege davontragen, und Pompeius Muscolus $^{67}$ war 3.559 Mal erfolgreich.

Bei diesen nahezu. unglaublichen Leistungen ${ }^{68}$ ist vorauszusetzen, dass die Wagenlenker jahre- bzw. jahrzehntelang an Rennen teilnahmen ${ }^{69}$, wobei entsprechend dem Angebot an Circusspielen ${ }^{70}$ manch einer, der in dieser Auflistung nicht herausragt, zu seiner Zeit der Rekordmann sein konnte ${ }^{71}$.

Die meisten Lenker waren im Laufe ihrer Karriere für mehrere oder alle vier Parteien ${ }^{72}$ an den Start gegangen und teilten so häufig ihre gesamten Siege nach factiones auf, für die sie sie erkämpft hatten ${ }^{73}$. P. Aelius Gutta Calpurnianus $^{74}$ hatte 102 Mal für die Weißen, 78 Mal für die Roten, 583 Mal für die Blauen und $364 \mathrm{Mal}$ für die Grünen siegen können ${ }^{75}$. M. Aurelius Polynices fuhr seine meisten Siege für die factio russata heraus ${ }^{76}$. Von seinen 739 Erfolgen fielen 655 auf die rote, 55 auf die grüne, 12 auf die blaue und

${ }^{67} \mathrm{CIL} 6,10048=\mathrm{ILS} 5287$.

${ }^{68}$ F. DREXEL, SG 4, 192 f. schreibt dem von Caracalla ermordeten Wagenlenker sogar 3782 Siege zu. A. CAMERON, Porphyrius, 179 n. 1, vergleicht antike mit neuzeitlichen Lenkern. Nach Ath. Pal. 16, 356 zählten byzantinische Wagenlenker ihre Erfolge in Hunderten.

${ }^{69} \mathrm{Vgl}$. zu der durchschnittlich im Jahr bestrittenen Rennen einzelner Wagenlenker J. P. V. D. BALSDON, Life, 322; A. HÖNLE/A HENZE, Amphitheater, 101.

70 Vgl. J. P. V. D. BALSDON, Panem et circenses, in: J. BIBAUW (Hrsg.), Hommages à MARCEL RENARD, Bd. 2, Brüssel 1969, 37 ff.; I. WEI LER, Sport, 247.

71 G. BIANCO, in: RLL 111, 1977, 323: „Scino, un auriga dell'età liberiana, in dodici o tredici anni di attività, consegne un numero irrisorio di vittorie rispetto ai campioni della successivia generazione; Fusco, all'incirca della stessa epoca, con 53 palme, è addirittura un primavista." 72 Vgl. A. CAMERON, Factions, 202, n. 4; J. P. V. D. BALSDON, Life, 315; I. WEILER, Sport, 248.

73 In der Inschrift des Wagenlenkers Epaphroditus (CIL 6, 10062=ILS 5282) sind auch acht Siege für die factio purpurea angeführt.

74 CIL 6,10047=ILS 5288; J.-P. THUILLIER, Sport, 106: „Gutta Calpurnianus, der sogar hippische Disziplinen im Verlauf des griechischen agon Capitolinus bestritten hat."

${ }^{75}$ Vgl. die übersichtliche Tabelle bei H. A. HARRIS, SGR, 202 f.; I. WEI LER, Sport, 247.

${ }^{76} \mathrm{CIL} 6,10049=$ ILS 5286. 
17 auf die weiße Partei ${ }^{77}$. Noch mehr Siege für die Roten erzielte Musclosus $^{78}$, bei dem von insgesamt 682 Siegen allein 672 auf die rote Partei fielen und die restlichen zehn Siege sich auf die anderen drei factiones verteilten. Da die Faktionen über die Leistungen ihrer Wagenlenker Akten führten ${ }^{79}$ war es ein Leichtes, eine Rangliste der erfolgreichsten Fahrer pro factio anzulegen ${ }^{80}$.

Ein weiterer Aspekt der Unterteilung der Siege bezieht sich auf die Zahl der am Rennen beteiligten Gespanne. Bei den meisten Rennen startete für jede factio ein Gespann (certamina singularum) ${ }^{81}$, und ein Erfolg in solch einem Rennen wurde am meisten geschätzt ${ }^{82}$. Verhältnismäßig häufig nahmen zwei Gespanne einer jeden Farbe an einem Rennen teil (certamina binarum) ${ }^{83}$. Relativ selten waren Rennen mit jeweils drei Vertretern einer Partei (certamina ternarum), und eine absolute Ausnahme müssen Wettkämpfe von Viererteams (certamina quaternarum) gewesen $\operatorname{sein}^{84}$. Eine genaue Aufstellung der Siege nach den eben angeführten Kriterien findet sich bei

\footnotetext{
77 Vgl. die übersichtliche Tabelle bei H. A. HARRIS, SGR, 207.

78 ClL 6, 10063=ILS 5281.

79 CIL 6, 10048=ILS 5287: „Actis continetur Avilium Teren factionis suae primum omnium vicisse ..." Vgl. L. BORSARI, in: BCAR 30, 1902, 183; H. A. HARRIS, SGR, 202; vgl. aber auch B. BALDWIN, in: Chiron 9, 1979, 189 ff.

${ }^{80}$ Vgl. L. BORSARI, in: BCAR 30, 1902, 183; A. CAMERON, Porphyrius, 15.

${ }^{81}$ Vgl. F. DREXEL, SG 4, 181.

82 Vgl. H. A. HARRIS, SGR, 198.

83 Hier sei darauf hingewiesen, dass Crescens insgesamt und Gutta Calpurianus in der Zahl seiner Erfolge für die grüne Partei mehr Siege im Zweierteam erzielt haben als in certamina singularum.

${ }^{84}$ Vgl. F. DREXEL, SG 4, 181. Fraglich bleibt, ob bei Rennen von Viererteams nur die beiden erfolgreichsten factiones mit jeweils vier Gespannen an den Start gingen, da z. B. der Circus Maximus nur über zwölf Startboxen verfügte; vgl. J. H. HUMPHREY, Circuses, 136 ff.
} 
P. Aelius Gutta Calpurnianus ${ }^{85}$. Dieser hatte z. B. für die rote Faktion im Einzelrennen 42, im Zweierteam 32, im Dreierteam drei und im Viererteam einen Sieg erzielen können. Weitaus erfolgreicher war er für die factio veneta, da er in ihren Diensten alleine 334, mit einem weiteren Lenker seiner Partei 184 und zusammen mit zwei Teamkollegen 65 Erfolge feiern konnte ${ }^{86}$. Da jedoch die Siege in den sogenannten Mannschaftsrennen weniger galten, weil durch ein taktisches Fahrverhalten der nachgestellten Lenker einer factio der Sieg des Stars erleichtert werden konnte, strich man besonders die Erfolge in certamina singularum heraus ${ }^{87}$. In der Inschrift des Wagenlenkers Diocles wird dies in mehrfacher Weise mittels Vergleich mit anderen berühmten Wagenlenkern gemacht ${ }^{88}$. So übertrifft Diocles den Wagenlenker Pontius Epaphroditus, der insgesamt zehn Siege mehr erzielen konnte als er, in der Zahl der Siege im Einzelrennen mit 1.064 zu 940. Im Vergleich zu Avilius Teres ist er diesem in der Zahl der Siege in certamina singularum überlegen, wo beide über hundert Siege im J ahr erzielen konnten. Schließlich führt man einen Vergleich mit Thallus, dem zuvor erfolgreichsten Lenker seiner Partei mit einem fremden Leitpferd (alieno principio) ${ }^{89}$ an, der wiederum Diocles als Rekordbrecher und neuen Rekordmann dastehen lässt, was auch unverhohlen zum Ausdruck gebracht wird:

"Adhuc augens gloriam tituli sui praecessit Thallum factionis suae, qui primus in factione russata [... at Dio]cles omnium agitatorum eminentissimus, quo anno alieno principio victor CXXXIIII, singularum vicit CXVIII; quo titulo praecessit omnium factionum

\footnotetext{
85 CIL 6, 10047=ILS 5288; vgl. J. P. V. D. BALSDON, Life, 322; A. HÖNLE/A HENZE, Amphitheater, 101.

${ }^{86}$ Vgl. die Tabellen bei F. DREXEL, SG 4, 181; H. A. HARRIS, SGR, 202 f.; I. WEILER, Sport, 247.

${ }^{87}$ Vgl.H. A. HARRIS, SGR, 198.

${ }^{88}$ Vgl. F. DREXEL, SG 4, 195.

${ }^{89}$ Vgl. F. DREXEL, SG 4,193.
} 
agitatores, qui umquam [certaminibus ludorum ci]rcensium interfuerunt. ${ }^{90}$

Viele Wagenlenker zeigten ihre Fahrkünste mit den verschiedensten Gespanntypen ${ }^{91}$. Zwar wurde das Viergespann am meisten genut女t und die damit erzielten Siege meist an erster Stelle aufgeführt, es ist jedoch festzuhalten, dass besonders häufig Rennen mit erhöhter Dotierung mit anderen Gespannformen als dem Viergespann bestritten wurden ${ }^{92}$. Dabei ist wahrscheinlich, dass nur besonders gute und erfahrene Lenker zu diesen Rennen zugelassen wurden ${ }^{93}$. Verständlicherweise wurden solche Siege besonders herausgestrichen. So erfahren wir, dass M. Aurelius Polynices mit dem Sechsgespann (seiuge) drei, mit dem Achtgespann (octoiugis) acht und mit dem Zehngespann (decemiuges) neun Siege erzielt hatte ${ }^{94}$. P. Aelius Gutta Calpurnianus hatte acht Siege mit dem Dreigespann (triga) vorzuweisen $^{95}$. Bei Diocles wurden besonders seine zwei Siege mit dem Sechsgespann - beide mit 60.000 Sesterzen dotiert - hervorgehoben, die er an einem Tag gewann, und in dieser Auflistung mehrerer Rekorde ${ }^{96}$ wurde

\footnotetext{
${ }^{90}$ Cl 6,10048=ILS 5287; vgl. A. GARCÍA Y BELLIDO, in: CAF 14, 1972, 5.

${ }^{91}$ Vgl. Appian. Mithr. 112 (Gespann mit sechzehn Pferden; E. SAGLIO, in: D-S 1 (1887), Fig. 1529 (Gespann mit zwanzig Pferden); J .-P. THUI LLIER, Sport, 105, Abb. 42.

92 H. A. HARRIS, SGR, 208.

93 Vgl. L. FRIEDLÄNDER, SG 2, 47; A. CAMERON, Porphyrius, 209 f.; J. P. V. D. BALSDON, Life, 316.

94 Cl L 6, 10049=ILS 5286; vgl. H. A. HARRIS, SGR, 207.

95 Cl 6, 10047; vgl. F. DREXEL, SG 4, 184.

96 A. GARCÍA Y BELLIDO, in: CAF 14, 1972, 16, n. 59: „Aunque la traducción no pueda hacerse con toda precisión, el sentido de este párrafo es claro, en general. Novis coactionibus es la parte más oscura. MOMMSEN la interpretó como comparationes victoriarum (principalmente con sus rivales). Friedländer la entendió como 'conquistas' o, apoyándose en el contexto, como 'combinaciones'. Creo que la versión más justa es la que nos proporciona hoy día la palabra inglesa record, corriente en todos los deportes para ejercicios en los que se llega a una nota sobresaliente, a alcanzar una 'marca', como también se dice empleando ya una voz castellana."
} 
noch der Sieg mit einem Siebengespann (septeiugis) erwähnt ${ }^{97}$, bei dem die Pferde miteinander nur durch Leinen verbunden waren ${ }^{98}$ :

\begin{abstract}
"Novis coactionibus et numquam ante titulis scriptis Diocles eminet, quod una die seiuges ad HS XL missus bis utrasque victor eminuit adque amplius ... suisque septem equis in se iunctis, numquam ante hoc numero equorum spectato ... ${ }^{199}$
\end{abstract}

Neben der überwiegenden Zahl der Rennen, die dem normalen Rennbetrieb zuzurechnen sind, kannte man eine kleine Zahl besonderer Rennen, die wahrscheinlich einem exklusiven Kreis von Fahrern vorbehalten waren ${ }^{100}$. Dazu zählte man das erste Rennen nach dem Festumzug (a pompa) ${ }^{101}$, Wettfahrten mit Gespannen, die noch keine Wettkampfpraxis hatten (equorum anagonum) ${ }^{102}$ und einen Wettbewerb, bei dem die Wagenlenker zu Beginn des Rennens zu ihren Gespannen laufen mussten (pedibus ad quadrigam) ${ }^{103}$. In solch ausgefallenen Wettfahrten war P. Aelius Gutta

97 Vgl. N. LEWIS \& M. REINHOLD, Roman Civilisation, Bd. 2, New York 1955, 231; H. A. HARRIS, SGR, 95.

98 A. CAMERON, Porphyrius, 210: „Obviously such off-beat contests - 'numquam ante tituli scripti', as they are called - were specially concocted to show off Diocles' unique skills."

99 CIL 6, 10048=ILS 5287; J.-P. THUILLIER, Sport, 105: „Diocles spricht selbst von „neuen Gespannformen“ (novae coactiones) und präzisiert dahingehend, daß ein Gespann mit sieben Pferden noch nie zu sehen war."

100 Vgl. F. DREXEL, SG 4,183; A. GARCÍA Y BELLIDO, in: CAF 14, 1972, 11.

${ }^{101}$ Vgl. H. S. VERSNEL, Triumphus. An Inquiry into the Origin, Development and Meaning of the Roman Triumph, Leiden 1970, $101 \mathrm{ff}$.

102 Vgl. J. P. V. D. BALSDON, Life, 316. H. A. HARRIS, SGR, 203, hält es auch für möglich, dass es sich dabei um Pferde handelt, die noch keine Siege erzielt hatten. Vgl. J.-P. THUILLIER, Sport, $105 \mathrm{f}$.

103 Vgl. D. MATZ, Pedibus ad quadrigam in Roman Chariot Racing, in: CW 79, 1985, 34ff. H. A. HARRIS, SGR, 204, trennt in der Inschrift in: CIL6, 10047=ILS 5288 die Angabe der Gewinnsumme nicht von der Zahl der Siege, wodurch er auf 61 Siege in diesen äußerst selten ausgetragenen Rennen kommt, was unwahrscheinlich ist. 
Calpurnianus besonders aktiv ${ }^{104}$, der mit seinen 45 Siegen in Rennen $a$ pompa scheinbar der Rekordmann in dieser Wettkampfform war ${ }^{105}$.

Ein weiterer Punkt der Unterteilung der Siege galt der Art und Weise, in der man gesiegt hatte ${ }^{106}$. Mit occupavit et vicit drückte man einen Start-Ziel-Sieg aus ${ }^{107}$, und einen Erfolg nach einer Aufholjagd führte man mit successit et vicit $\mathrm{an}^{108}$. Am meisten geschätzt waren die Siege, bei denen man dem führenden Wagenlenker auf der Zielgeraden noch den Sieg entrissen hatte, was mit eripuit et vicit ausgedrückt ${ }^{109}$ und z. T. mit der Angabe ergänzt wurde, welche factio man so knapp hatte besiegen können ${ }^{110}$. Auch wurden in dieser Hinsicht Vergleiche angestellt und neue Bestmarken mitgeteilt: Ein Wagenlenker der blauen Partei hielt den Jahresrekord mit 51 Siegen eripuit et vicit, Teres war mit 52 Siegen erfolgreicher, ${ }^{111}$ Pontius Epaphroditus führte

${ }^{104} \mathrm{CIL} 6,10047=I L S 5288$.

105 In der Inschrift des Diocles (CIL 6, 10048=ILS 5287) wird nur die Zahl der Rennen $a$ pompa angegeben, an denen er teilgenommen hatte, nicht aber, wie oft er darin gesiegt hatte.

106 Vgl. H. A. HARRIS, SGR, 199 f., zu dem schwer zu deutenden Ausdruck praemissus et vicit.

107 Vgl. F. DREXEL, SG 4, 191; J. REGNER, in: RE Suppl. 7 (1940), s. v. Ludi circenses, 1638; J. P. V. D. BALSDON, Life, 318; H. A. HARRIS, SGR, 199: ,.... took the lead the start and held it throughout."

108 Vgl. L. FRIEDLÄNDER, SG 2, 49; F. DREXEL, SG 4, 191; H. A. HARRIS, SGR, 199: ,... came from the rear to win."

109 Vgl. F. DREXEL, SG 4, 191; N. LEWIS \& M. REINHOLD, Civilisation, 231; J. P. V. D. BALSDON, Life, 318; H. A. HARRIS, SGR, 199: "Lay second throughout the race and snatched victory in the last stages." Vgl. auch zum Stellenwert dieser Siege A. CAMERON, Porphyrius, 243; DERS., Factions, 71.

110 Vgl. F. DREXEL, SG 4, 191; H. A. HARRIS, SGR, 199; A. CAMERON, Porphyrius, 80 f.; DERS., Factions, $63 \mathrm{f}$.

${ }^{111}$ CIL 6, 33937; vgl. L. BORSARI, in: BCAR 30, 1902, 179. 
in der Gesamtzahl der Siege eripuit et vicit mit 467 Erfolgen, und Diocles übertraf inn um 35 Siege dieser Art $^{112}$.

Nicht nur die Siege wurden von vielen Lenkern angeführt, sondern auch die sonstigen Platzierungen ${ }^{113}$. Knapp konnte dies wie bei einem uns unbekannten Lenker der Grünen mit ,... ad honorem venit CCCLI/I/“114 und wie bei Teres mit ,.... ad honorem venit $\infty \infty D D C L V^{\prime 115}$ ausgedrückt sein. Meist war es jedoch genauer wiedergegeben. Von Scirtus erfahren wir, dass er mit dem Viergespann neben elf Siegen (davon vier im Wiederholungslauf) 39 zweite und 60 dritte Plätze herausfuhr ${ }^{116}$. In der Inschrift eines unbekannten Lenkers der grünen Partei sind dessen 354 Siege und Platzierungen nach Rennen mit dem Vier- und Zweigespann unterteilt ${ }^{117}$. Bei Crescens, der 47 erste, 130 zweite und 111 dritte Plätze verbuchen konnte, können wir durch Subtraktion von der Summe seiner 686 Starts ermitteln, dass er sich $398 \mathrm{Mal}$ nicht platzieren konnte ${ }^{118}$. Noch präziser sind die Angaben in der Diocles-Inschrift, in der zu Anfang schon die Zahl der Starts und Siege angeführt worden war und dann folgt:

"Ad honorem venit $\infty \infty$ oo DCCC. Tulit secundas DCCCLXI, tertias $D L X X V I$, quartas ad HS $\infty$ I, frustra exit oo CCCLI'119.

112 CIL 6, 10048=ILS 5287. F. DREXEL, SG 4, 195: „Nach den größten Wagenlenkern der Vergangenheit wird hier der größte der Gegenwart mit Diocles verglichen." Vgl. auch H. A. HARRIS, SGR, 200.

113 Vgl. L. FRIEDLÄNDER, SG 2, 46.

${ }^{114} \mathrm{CIL} 6,10055=$ ILS 5284.

${ }^{115} \mathrm{ClL} 6,10048=$ ILS 5287.

${ }^{116}$ CIL 6,10051=ILS 5283; vgl. H. A. HARRIS, SGR, 204.

${ }^{117} \mathrm{ClL} 6,10055=$ ILS 5284.

118 CIL 6,10050=ILS 5285; vgl. I. WEILER, Sport, 247.

119 CL 6, 10048=ILS 5287. In dieser Inschrift finden wir den einzigen Beleg für die Honorierung eines vierten Platzes mit 1000 Sesterzen; vgl. A. GARCÍA Y BELLIDO, in: CAF 14, 1972, 12. 
Es ist nicht auszuschließen, dass bei dem großen Interesse an Statistik und der ständigen Suche einiger Wagenlenker nach Bestmarken neben dem absolut erfolgreichsten auch der relativ erfolgreichste Lenker gesucht wurde. Nicht nur die Erfolge der Lenker, sondern auch die der Pferde ${ }^{120}$ wurden genauestens festgehalten ${ }^{121}$. Besonders ausführlich werden wir durch zwei Listen, die den Inschriften zweier sehr siegreicher Wagenlenker entstammen, über die Leistungen von 74 bzw. 122 Rennpferden unterrichtet ${ }^{122}$. Nach Name ${ }^{123}$ und Herkunft ${ }^{124}$ der Pferde ist einem jeden Renner die Zahl seiner Siege beigegeben, wobei sich in der ersten Liste ${ }^{125}$ die Zahl der Siege von 1 bis 32, in der zweiten Liste ${ }^{126}$ von 1 bis 152 bewegt. Kürzer fasst sich P. Aelius Gutta Calpurnianus in der von inm selbst gesetzten Inschrift ${ }^{127}$. Er führt neben Farbe ${ }^{128}$ und Herkunft die Zahl der Siege von jeweils nur vier Rennpferden an, mit denen er bei den Blauen und Grünen besonders erfolgreich war:

${ }^{120}$ Zur Aufzucht von Rennpferden vgl. A. HÖRSCHEMEYER, Die Pferdezucht im Klassischen Altertum, Diss. Gießen, 1928; zu Stammbäumen von Pferden vgl. Marx. 3,63,12; Juv. 8,62 f.; Stat. 5,2,22 ff; zu Brandzeichen vgl. A. BALIL, in: BRAH 151, 1962, 336 ff.

121 Vgl. G. BIANCO, in: RTL 111, 1977, 314; vgl. Mart. 10,9,5, zu dem Superrenner Andraemon; vgl. Suet. Calig 55 zum Rennpferd Incitatus, das einen Stall aus Marmor und eine Krippe aus Elfenbein hatte; vgl. Dio Cass. 56,6 und 61,6 zur Versorgung ausgemusterter Pferde; vgl. J. P. V. D. BALSDON, Life, 318, zum Doping von Rennpferden. 122 Vgl. zur wichtigsten Position in einem Gespann L. FRIEDLÄNDER, SG 2, 32; H. A. HARRIS, SGR, 171.

123 Vgl. J. M. C. TOYNBEE, in: PBSR 16, 1948, 26 ff; DIES., Tierwelt, 163 ff; A. HÖNLE/A. HENZE, Amphitheater, $97 \mathrm{f}$.

${ }^{124}$ Vgl. L. FRIEDLÄNDER, SG 2, 30; A. BALIL, in: BRAH 151, 1962, 338 ff. P. VIGNERON, Cheval, $128 \mathrm{ff}$.

${ }^{125}$ Cl L 6, 10053; vgl. Cl L 6, 33937; L. BORSARI, in: BCAR 30, 1902, $177 \mathrm{ff}$.

${ }^{126} \mathrm{CIL} 6,10056=$ ILS 5290.

127 CIL 6, 10047=ILS 5288.

${ }^{128}$ Vgl. H. A. HARRIS, SGR, 202; J . M. C. TOYNBEE, Tierwelt, 164. 
"Germinatore n. Af. LXXXXII, Silvano r. Af. CV, Nitido git. Af. LII, Saxone n. Af. LX..., Danao b. Af. XIX, Oceano n. CCVIIII, Victor $r$. cCCCXXIX, Vindice b. CLVII. 'A29

In einer Inschrift, zu der die Darstellung eines Mannes mit zwei Pferden gehört, ${ }^{130}$ werden neben den Siegen auch die Platzierungen zweier sehr erfolgreicher Pferde angeführt:

"Aquilo n. K. Aquilonis, vicit CXXX, secund. tulit $L X X X V I I I$, ter. tul. $X X X Y I I$, Hirpinus $n$. Aquilonis, vicit CXIIII, secundas tulit $L X I$, tert. tul. $X X X V{ }^{، 131}$

Die beiden zuletzt angeführten Pferde zählten zu der Klasse der centenarii, d. h. zu jener Gruppe von Rennpferden, die hundert und mehr Siege erzielt hatten ${ }^{132}$, wobei man auch ducenarii kannte, also Pferde, die zweihundert oder mehr Siege erkämpften ${ }^{133}$. So priesen sich Lenker, möglichst viele Rennpferde zu solchen Erfolgen geführt zu haben: „Equos centenarios fecit $N$ VIIII et ducenar. I. ‘34

129 ClL 6, 10047=ILS 5288; vgl. A. HÖNLE/A HENZE, Amphitheater, 101. Vgl. A. CAMERON, Porphyrius, 47 f., zu den 429 Siegen des Pferdes Victor.

${ }^{130}$ Vgl. G. V. GENTIII, Le gare del circo nel mosaico di Piazza Armerina, in: Bolletino d'Arte 17, 1957, 7 ff.; L. VOGEL, Circus Race Scenes in the Early Roman Empire, in: The Art Bulletin 51, 1969, $155 \mathrm{ff}$; A. HÖNLE/A HENZE, Amphitheater, bes. $92 \mathrm{ff}$;; K. M. D. DUNBABIN, in: AJA 86, 1982, 65 ff. u. pl. 5 ff.

${ }^{131} \mathrm{ClL} 6,10069=$ ILS 5295.

132 Vgl. J. REGNER, in: RE Suppl. 7 (1940), s. v. Ludi circenses, 1640; H. A. HARRIS, SGR, 201.

133 Vgl. L. FRIEDLÄNDER, SG 2, 28; A. GARCíA Y BELLIDO, in: CAF 14, 1972, 13.

${ }^{134}$ CIL 6, 10048=I LS 5287; vgl. F. DREXEL, SG 4, 192; I. WEI LER, Sport, 247. 
Auch konnte ein Wagenlenker damit prahlen, dass er mit bestimmten Pferden besonders viel Geld bzw. Sonderprämien eingefahren hatte ${ }^{135}$. Hierin muss Diocles all seine Kollegen übertroffen haben:

„Nobilissimo titulo Diocles nitet, cum Fortunatus factionis prasinae, in victore Tusco victor CLII, $L$ vicit $X, L X I$. 1336

Ferner wurden Vergleiche angestellt bezüglich der größten Erfolge mit einem fremden Leitpferd (alieno principo), ${ }^{137}$ oder eine Bestmarke wurde gesucht hinsichtlich der höchsten Zahl an Siegen mit den wenigsten J ochpferden ${ }^{138}$ :

"Ampliatis titulis suis, Cotyno Galata Abigeio Lucido Pompeiano
introiugis quinque victor CCCCXXXXV, singularum vicit
CCCLXXXXXII. ‘39

Auf der Suche nach Rekorden war man erfinderisch und ermittelte Bestmarken durch Kombination mehrerer Sonderheiten ${ }^{140}$ :

"Omnium admiratione merito notatum est, quod uno anno alieno principio, duobus introiugis Cotyno et Pompeiano, vicit $L X X X X V I I I I$, $\angle X I, L X X X, X L I, X X X I I$. ${ }^{141}$

135 Der Wagenlenker Diocles hatte etwa zehn Prozent seiner Gesamtgewinnsumme durch Sonderprämien erzielt.

${ }^{136}$ CIL 6, 10048=ILS 5287; vgl. H. A. HARRIS, SGR, 201.

137 Vgl. A. GARCíA Y BELLIDO, in: CAF 14, 1972, 14.

138 Vgl. L. BORSARI, in: BCAR 30, 1902, 179.

${ }^{139} \mathrm{Cl}$ 6, 10048=ILS 5287; vgl. F. DREXEL, SG 4, 195.

140 Vgl. H. A. HARRIS, SGR, 200.

141 CIL 6, 10048=ILS 5287; H. A. HARRIS, SGR, 201: „One pair of these yoke-horses, Pompeianus and Cotynus, running together, won 99 times, the earliest record in any sport of a century so narrowly missed." 
Im Kreise der Wagenlenker muss es von gewisser Wertschätzung gewesen sein, möglichst viele Siege mit afrikanischen Pferden erzielt zu haben ${ }^{142}$. Ein uns unbekannter miliarius hatte seine 1.378 Siege mit afrikanischen Pferden - neben 584 Erfolgen mit spanischen Pferden - besonders hervorgehoben ${ }^{143}$. Am erfolgreichsten mit afrikanischen introiuges muss aber Diocles gewesen sein:
„Inter eminentes agitatores introiugis Afris plurinum vicerunt: Pontius Epaphroditus factionis Venetae in Bubalo vicit CXXXIIII, Pompeius Musclosus factionis prasinae in ... vicit CXV, Diocles superatis eis, in Pompeiano victor CLII, singularum vicit CXXXXIIII. 144

$\mathrm{Da}$ in gewissem Grad die Gesamtgewinnsumme ein Spiegelbild einer siegreichen Sportlerkarriere war ${ }^{145}$, versäumten es manche Lenker nicht, bis auf den letzten Sesterzen ihre gesamten Renneinnahmen ${ }^{146}$ anzugeben, wie wir es von Crescens erfahren: Er gewann 1.558.346 Sesterzen. ${ }^{147}$ Weitaus mehr Geld gewann Diocles, der allein an Sonderprämien mehr als das

\footnotetext{
142 Vgl. H. A. HARRIS, SGR, 201; M. FLORIANI SQUARCIAPINO, in: RAL 34, 1978, 280.

${ }^{143} \mathrm{CIL}$ 6,10056; vgl. H. A. HARRIS, SGR, 202.

144 ClL 6, 10048=ILS 5287; H. A. HARRIS, SGR, 201: „The oddest record claimed by Diocles is that he was the best driver of African horses."

145 Nach Martial (10,74,5 f.) hatte der unter Domitian berühmte Wagenlenker Scorpus in einer Stunde fünfzehn Beutel Gold gewinnen können, und Juvenal (7,113 f.) schätzte den Verdienst eines Wagenlenkers der roten Partei gleich dem von hundert Rechtsanwälten. Sueton (Calig. 55) berichtet, dass Caligula dem Wagenlenker Eutychus bei einem Gelage 2 Millionen Sesterzen geschenkt hat.

${ }^{146}$ Es gab auch wertvolle Sachpreise: vgl. CIL 6, 2065 („victores palmis et coronis argenteis honoravit“); Symmach. epist. 5,20,2 (holostericae vestes).

147 ClL 6, 10050=ILS 5285; vgl. E. LOVATELLI CAETANI, in: BCAR 30, 1902, 171; J. P. V. D. BALSDON, Life, 322; I. WEILER, Sport, 247.
} 
Doppelte verdiente, was Crescens insgesamt gewann. ${ }^{148}$, und dessen Gesamtgewinnsumme sich auf 35.863.120 Sesterzen belief ${ }^{149}$.

Besondere Aufmerksamkeit wurde den Rennen mit erhöhter Dotierung geschenkt, die vermutlich den besten Fahrern vorbehalten blieben ${ }^{150}$. Die praemia maiora ${ }^{151}$ setzten bei 30.000 Sesterzen ein ${ }^{152}$ und erstreckte sich über 40.000 und 50.000 bis zur Höchstdotierung von 60.000 Sesterzen für den Sieger pro Rennen ${ }^{153}$.

M. Aurelius Polynices konnte die Sonderprämie von 40.000 Sesterzen dreimal, die von 30.000 Sesterzen 26 Mal, M. Aurelius Mollicius Tatianus die von 40.000 Sesterzen zweimal gewinnen ${ }^{154}$. Die Inschrift des P. Aelius Gutta Calpurnianus führt 67 Sonderprämien bei 1.127 Siegen $a^{155}$. Für erfolgreiche Wagenlenker eröffneten sich besonders im zweiten J ahrhundert n. Chr. unglaubliche Möglichkeiten ${ }^{156}$.

Besonders im Bereich des Preisgeldes wurden Höchstleistungen gesucht und verzeichnet ${ }^{157}$. Hierzu mögen drei Beispiele aus der dafür sehr ergiebigen Inschrift des Diocles angeführt $\operatorname{sein}^{158}$. Dieser übertraf einen uns

\footnotetext{
${ }^{148}$ Vgl. die Aufstellung bei F. DREXEL, SG 4, 189; vgl. auch L. BORSARI, in: BCAR 30, 1902, 181.

149 CIL 6, 10048=ILS 5287; vgl. A. GARCÍA Y BELLIDO, in: CAF 14, 1972, 6.

${ }^{150}$ Vgl. F. DREXEL, SG 4, 182.

151 Die praemia maiora für Siege in Rennen mit dem Dreigespann müssen niedriger gewesen sein, vgl. F. DREXEL, SG 4, 183.

152 Vgl. H. A. HARRIS, SGR, 199.

${ }^{153}$ Vgl. J. P. V. D. BALSDON, Life, 318; A. CAMERON, Factions, 63.

${ }^{154} \mathrm{CIL}$ 6, 10049=I LS 5286; vgl. A. CAMERON, Porphyrius, $156 \mathrm{ff}$.

155 ClL 6,10047=ILS 5288; vgl. A. HÖNLE/A HENZE, Amphitheater, 101.

${ }^{156} \mathrm{ClL}$ 6, 10048=ILS 5287; vgl. F. DREXEL, SG 4, 182.

157 Vgl. A. GARCÍA Y BELLIDO, in: CAF 14, 1972, 12; A. CAMERON, Porphyrius, 206; R. SYME, in: AJAH 2, 1977, 86.

${ }^{158}$ CIL 6, 10048=ILS 5287; vgl. H. A. HARRIS, SGR, 200.
} 
unbekannten Lenker der Grünen, der als erster seit Gründung der Stadt 50.000 Sesterzen siebenmal gewinnen konnte, allein mit drei introiugi:

„... factionis prasinae, victor $\infty \times X V$, primus omnium urbis conditae ad HSL vicit VII, Diocles praecedens eum introiugis tribus Abigeio Lucido Parato L vicit VIII. ${ }^{1159}$

Nachdem Diocles überragende, durch viele Bestleistungen bestätigte Ausnahmestellung $^{160}$ gegenüber einzelnen - ehemaligen und zu seinen Lebzeiten aktiven - Fahrern sowohl der eigenen factio als auch der anderen Parteien herausgearbeitet worden war $^{161}$, stellte man seine Leistungen gegen die zusammengefassten Erfolge gleich mehrerer Spitzenfahrer ${ }^{162}$. Drei Wagenlenker der Grünen, alles miliarii, hatten die praemia maiora von 50.000 Sesterzen elfmal einstreichen können. Diocles überbot sie darin allein mit den beiden Jochpferden Pompeianus und Lucidus ${ }^{163}$. In Bezug auf Erfolge in außerordentlich hoch dotierten Rennen konnte sich Diocles sogar mit der zusammengefassten Leistung dreier berühmter und äußerst erfolgreicher Lenker verschiedener Parteien messen ${ }^{164}$. Diese drei Wagenlenker - unter innen Flavius Scorpus ${ }^{165}$ - hatten zusammen 6.632

${ }^{159} \mathrm{Cl}$ L 6, 10048=ILS 5287; vgl. A. CAMERON, Porphyrius, 179 („money jock”).

160 N. LEWIS \& M. REINHOLD, Civilisation, 232: „The remainder of the inscription (about three fifth), under the heading 'His Records', lists in great detail, naming record-holding drivers and horses, the various records which Diocles broke: 'the champion of all charioteers ... he excelled the charioteers of all the stables who ever participated in the races of the circus games."

${ }^{161}$ Vgl. F. DREXEL, SG 4, 192.

162 Vgl. F. DREXEL, SG 4, 194.

163 Vgl.H. A. HARRIS, SGR, 201.

${ }^{164}$ Vgl. A. GARCíA Y BELLIDO, in: CAF 14, 1972, 15 f.; A. CAMERON, Porphyrius, 206.

165 Zu Ruhm und Beliebtheit des Wagenlenkers Scorpus vgl. Mart. 5,25,9 f. und 10,50,53. F. CUMONT, Recherches sur le symbolisme funéraire des Romains, Paris 1942, 458: "Ce Scorpus, on l'a depuis longtemps reconnu, était un aurige fameux du temps de Domitien. 
Siege errungen, davon 28 in Rennen von 50.000 Sesterzen. Diocles allein war bei nur 1.462 Siegen 29 Mal erfolgreich darin gewesen:

„... factionis prasinae victor $\infty \times X V$ et Flavius Scorpus, victor III $X L V I I$, et Pompeius Musclosus, victor ĪII DLVIIII, tres agitatores victores VI DCXXXII, ad HS L vicerunt XXVIII, at Diocles omnium agitatorum eminentissimus, victor oo CCCCLXII, L vicit XXVIIII'166.

Diese enormen Gewinnsummen ${ }^{167}$ drücken auch den hohen Stellenwert und Beliebtheitsgrad der Wagenrennen im antiken Sport aus ${ }^{168}$ und boten den erfolgreichen Lenkern ${ }^{169}$ neben beträchtlichen Einkünften ${ }^{170}$ zahlreiche Möglichkeiten zur Darstellung ihrer Leistungen.

Ferner führten auch die römischen agitatores die von den griechischen Wagenlenkern her bekannten Kriterien besonderer Erfolge für sich und ihre

Martial poète parle, non sans quelque envie, des gains fabuleux réalisés par ce champion sportif, qui avait battu tous les records."

${ }^{166}$ ClL 6,10048=ILS 5287; vgl. H. A. HARRIS, SGR, 200.

167 Ungeklärt ist, wieviel die Lenker von der Gewinnsumme an ihre Rennställe abführen mussten; vgl. A. GARCÍA Y BELLIDO, in: CAF 14, 1972, 10; A. CAMERON, Factions, 16 f.; E. RAWSON, in: PBSR 36, 1981, 12; J. H. HUMPHREY, Circuses, 517.

168 Vgl. L. FRIEDLÄNDER, SG 2, $25 \mathrm{f}$.

${ }^{169}$ Zur sozialen Herkunft der Wagenlenker vgl. J. REGNER, in: RE Suppl. 7 (1940), s. v. Ludi circenses, 1633 f.; H. A. HARRIS, in: PVS 8, 1968-1969, 17; DERS., SGR, 153 u. 207 f.; A. CAMERON, Factions, 202; E. RAWSON, in: PBSR 36, 1981, 16; J. P. THUILLIER, Jeux athlétiques, $510 \mathrm{ff} ; \mathrm{J}$. H. HUMPHREY, Circuses, $137 \mathrm{f}$.

170 Vgl. L. FRIEDLÄNDER, SG 2, 26; W. M. GREEN, in: AJPh 51, 1930, 2491; H. A. HARRIS, SGR, 2071; R. PALMIERI, in: RAAN 53, 1978, 57 ff. ClL 6, 10058=ILS 5296: „M. Aurelio Libero patri et magistro et socio, domino et agitatori factionis prasinae." Zu einem solchen Aufstieg vom Wagenlenker zum Mitglied des Vorstandes einer factio vgl. G. RODENWALDT, in: JDAl 55, 1940, 12 ff. 
Pferde $\mathrm{an}^{171}$. Einige Lenker verwiesen bei der Darstellung ihrer Rekorde auf deren Erst- und Einmaligkeit ${ }^{172}$, hoben ihre Spitzenposition in der Rangliste der Lenker hervor ${ }^{173}$, stellten sich als den Konkurrenten überlegen dar ${ }^{174}$ und übertrafen an Ruhm sogar einen Halbgott ${ }^{175}$. Die von innen gelenkten Pferde waren unbesiegt ${ }^{176}$ oder den anderen Pferden weit überlegen ${ }^{177}$.

Die römischen Wagenlenker maßen ihre Leistungen besonders hinsichtlich der Zahl der Siege, der von innen zum Erfolg geführten Pferde und der von innen erzielten Preisgelder. Dies konnte in jeder Sparte bei sehr speziellen Merkmalen - z. B. gegliedert nach factiones, Teilnahmerzahl, Gespannform, etc. - bis zur Kombination all dieser Kriterien gehen. Bei der Darstellung der Leistungen vieler römischer Wagenlenker wird das Bestreben deutlich, eindrucksvolle Leistungen für den einen oder anderen agitator zu beanspruchen, was z. T. auch explizit zum Ausdruck gebracht wurde. Häufig findet man den Versuch, durch Vergleiche mit Kollegen nicht nur der eigenen factio, sondern auch mit Lenkern früherer Zeiten und anderer Parteien mitunter mehrerer Lenker gleichzeitig - Bestleistungen zu markieren. Hierbei kann man sich des Eindrucks nicht erwehren, dass man teilweise durch vage

171 Mehr Parallelen in der Darstellung von besonderen Leistungen nach Art griechischer Wagenlenker finden sich bei den byzantinischen Wagenlenkern; vgl. A. CAMERON, Porphyrius, 85 u. 156.

172 Vgl. ClL 6, 10048=ILS 5287 („Solus et primus omnium agitatorum“); Fuscus (CIL 6, 33950) war der erste, der bei seiner ersten Teilnahme auch gleich siegte; vgl. H. A. HARRIS, SGR, 206. Vgl. zu den byzantinischen Lenkern Anth. Pal. 16, 340 u. 376; vgl. A. CAMERON, Porphyrius, 86, 121 u. 166.

$173 \mathrm{CIL} 6,10048=$ I LS 5287 („I nter miliarios agitatores primum locum obtinere ...").

174 Vgl. W. PEEK, in: ZPE 42, 1981, 287 f.; Vgl. auch Anth. Pal. 15,43, zu dem byzantinischen Wagenlenker Konstantinos, der in der J ugend den Alten, im Alter den J ungen überlegen war.

175 W. PEEK, Griechische Vers-Inschriften I, Berlin 1955, Nr. 1844; vgl W. BACKHAUS, GL 2, 214. Vgl. auch Anth. Pal. 15,48, wo der Lenker Uranios mit einem Gott gleichgesetzt wird.

176 CIL 6, 10082; vgl. H. A. HARRIS, SGR, $211 \mathrm{f}$.

${ }^{177}$ Vgl. W. PEEK, Vers-Inschriften I, Nr. 1844; DERS., in: ZPE 42, 1981, 287 f. 
konstruierte Vergleiche und dem Herausstreichen von Sonderheiten gezielt neue Rekorde suchte. In besonders auffälliger Weise treten diese Aspekte in der Inschrift des Diocles zutage, wo man nahezu eine Rekordmanie unterstellen kann.

In gewissem Grade erfuhr diese Entwicklung im byzantinischen Circusbetrieb noch eine Steigerung. Zwar haben wir von byzantinischen Wagenlenkern im Einzelnen nicht so umfangreiche und detaillierte Angaben über ihre Leistungen, wie wir es von einigen römischen agitatores besitzen, doch im Auflisten brillanter Leistungen wie im Herausstellen von Rekorden stehen sie ihren römischen Kollegen nicht nach. Es hat sogar den Anschein, dass sie in der Zahl der Starts und Siege, den Verdienstmöglichkeiten und vor allem in Ruhm und Ansehen bei Herrscherhaus und Volk die römischen Lenker übertrafen. 
3.

Charakteristika

Im zweiten Teil unserer Arbeit gehen wir an die Auswertung der zuvor gewonnenen Erkenntnisse, die es uns nun ermöglichen, die Charakteristika des Sports der griechisch-römischen Antike zu bestimmen.

\section{1. $\quad$ Öffentlichkeit}

Sportliche Veranstaltungen waren ein fester und wichtiger Bestandteil des gesellschaftlichen Lebens in der griechisch-römischen Antike. ${ }^{1}$ Die (Schau-) Spiele wurden einst zu Ehren und zur Verherrlichung der Götter in zyklisch wiederkehrenden Festen durchgeführt ${ }^{2}$. Die religiös-kultischen Inhalte dieser Feste $^{3}$ - in den Anfängen noch von primärer Bedeutung - rückten im Laufe der Zeit zunehmend in den Hintergrund, ohne ganz (zumindest formal) abgeschafft oder verdrängt zu werden. ${ }^{4}$ Gemeinsame Gebete und Opfer ${ }^{5}$ bei den Olympischen Spielen riefen immer wieder den sakralen Charakter der Spiele in Erinnerung, ${ }^{6}$ konnten aber eine stetige Säkularisierung nicht aufhalten ${ }^{7}$. Auch die circensischen Spiele zeigten noch deutlich religiös-

\footnotetext{
${ }^{1}$ Vgl. B. BI LINSKI, Agoni, 87 ff.; I. WEI LER, Sport, 78 ff.; D. SANSONE, Genesis, 76: „When we consider sport in ancient Greece, the first thing that strikes us ist the remarkable importance that sport assumed in the life and thought oft the Greeks."

${ }^{2}$ Vgl. W. DECKER, Sport, $32 \mathrm{ff}$.

${ }^{3}$ N. B. CROWTHER, in: Athletica, 3: „Although the Greeks regularly practiced sport in sacred places, the Games were not especially religious in a society where religion was ingrained in every aspect of life."

${ }^{4}$ Vgl. J.-M. ANDRÉ, Fest, 36.;R. MANDELL, Sport, 117: „Obwohl die traditionellen Zeremonien zynisch beobachtet wurden und die Wettkämpfe jetzt keinerlei Verbindung mehr zu den lebendigen spirituellen Interessen der Griechen und Römer hatten, ließen ihr Reiz und ihr Ansehen die Feste doch fortbestehen."

${ }^{5}$ Vgl. W. E. SWEET, 208, Abb. 80; D. G. KYLE, Spectacle, 115 Fig. 6.1.

${ }^{6}$ L. DEUBNER, Kult und Spiel im alten Olympia, 1936, 7: „Die Olympischen Spiele waren heilige Spiele an heiligem Ort zu heiliger Festzeit veranstaltet, ein kultischer Akt zu Ehren der Gottheit.“

${ }^{7}$ Vgl. I. WEI LER, Sport, 124.
} 
kultische Züge, ${ }^{8}$ vor allem bei der pompa, ${ }^{9}$ der feierlichen Prozession vom Capitol über das Forum Romanumzum Circus Maximus. ${ }^{10}$

Die Olympischen Spiele wie auch andere panhellenische Feste waren Ereignisse von großem öffentlichen Interesse. Schon im 5. Jahrhundert v. Chr. kamen zu diesem Fest - trotz z. T. äußerst widriger Umstände ${ }^{11}$ - um die 40.000 Besucher $^{12}$. Noch höhere Zuschauerzahlen finden wir bei den munera im Colosseum (mit einem Fassungsvermögen von 50.000 Zuschauern) ${ }^{13}$ und bei den Wagenrennen im Circus Maximus (mit einer Zuschauerkapazität von 250.000) ${ }^{14}$. Um große Menschenmassen als Zuschauer für die Veranstaltungen zu gewinnen, wurden im antiken Griechenland zur Aufforderung und Einladung zu den Olympischen Spielen Herolde ausgesandt. ${ }^{15}$ Die für diesen Zeitraum ausgesprochene Friedenspflicht mag neben den spannenden Wettkämpfen ein weiteres Moment für eine gesteigerte Anziehungskraft gewesen $\operatorname{sein}^{16}$. Im Römischen Reich waren es professionelle Plakatschreiber, die Werbung für die Spiele

8 Bei Verstößen gegen rituelle Vorschriften war eine Wiederholung der Prozession (instauratio) erforderlich. Vgl. D. P. HARMON, The Religious Significance of Games in the Roman Age, in: W. RASCHKE (Hrsg.), Archaeology, 236-255.

${ }^{9} \mathrm{Vgl} \mathrm{H}$. S. VERSNEL, Triumphus. An Inquiry into the Origin, Development, and Meaning of the Roman Triumph, London 1970, bes. 101-114. I. WEI LER, Sport, 240.

${ }^{10}$ E. KÜNZEL, Der römische Triumpf. Siegesfeiern im antiken Rom, München 1988, 105ff. “

${ }^{11}$ Paus. 5,14,1; Th. KLEMMER, Siegerkranz, 69: „Die sanitären Voraussetzungen müssen zur antiken Zeit katrastrophal gewesen sein. Latrinen sind erst für die Römer, und auch nur in geringer Anzahl, belegt. Zudem denke man an das Blut der Opfertiere, welches sich unkontrolliert in riesigen Pfützen am Zeusaltar sammelte und in die Erde versickerte."

${ }^{12}$ Vgl.I WEILER, Sport, 236; H. KYRIELEIS, Olympia, 111; W. DECKER, Sport, $103 \mathrm{f}$.

${ }^{13} \mathrm{Vgl}$. A. HÖNLE/A. HENZE, Amphitheater, 37; P. CONNOLLY, Colosseum, 53 u. 57.

14 W. BACKHAUS, GL 2, 220; A. HÖNLE/A.HENZE, Amphtheater, 126; J. H. HUMPHREY, Circuses, $126 \mathrm{ff}$.

${ }^{15}$ Vgl. V. OLIVOVÁ, Sport, 117.

${ }^{16} \mathrm{Vgl}$. M. LÄMMER, Der sogenannte olympische Friede in der griechischen Antike, in: Stadion 8-9, 1982-1983, $47 \mathrm{ff}$. 
machten ${ }^{17}$, soweit nicht schon durch den geläufig bekannten Festkalender oder Mundpropaganda die Termine bekannt waren. ${ }^{18}$ Auch war nicht nur in Zeiten der Pax Romana weitestgehend für Sicherheit bei den Reisen zu den Austragungsstätten gesorgt. ${ }^{19}$ Das Kernstück der Veranstaltungen waren die sportlichen Wettkämpfe. Die Masse der Zuschauer war gekommen, um gute und spannende Wettkämpfe zu erleben, manche von innen auch in der Hoffnung, dass Bekannte oder Landsleute erfolgreich abschnitten. ${ }^{20}$ Dem antiken Sportpublikum kann man Sachkenntnis und eine faire Einstellung attestieren. ${ }^{21}$ Die Reaktionen der Zuschauer fielen entsprechend der Leistungen aus, und Krawalle (wie etwa in Pompeji 59 n. Chr.) blieben die Ausnahme. ${ }^{22}$ Neben dem sportlichen Geschehen galt auch dem gesamten Umfeld solcher Großveranstaltungen gesteigertes Interesse. Für das leibliche Wohl sorgten zahlreiche Stände mit Essen und Getränken, ${ }^{23}$ Musiker und Artisten ${ }^{24}$ sorgten für Unterhaltung, Wahrsagerinnen ${ }^{25}$ und Dirnen ${ }^{26}$ boten ihre Dienste feil. Philosophen und Schriftsteller ${ }^{27}$ lasen aus ihren neuesten Werken, Bildhauer und Maler suchten die Aufmerksamkeit der Besucher zu

\footnotetext{
${ }^{17}$ Vgl. J. COLIN, in: Pallas 4, 1956, 51 ff; K.-W. WEEBER, Wahlkampf im alten Rom, Düsseldorf 2007, $81 \mathrm{ff}$.

${ }^{18}$ Vgl. H. H. SCULLARD, Römische Feste, Kalender und Kult, Mainz 1985, 56. ff.

${ }^{19}$ Vgl. J.-M. ANDRÉ, Feste, 220.

${ }^{20}$ Vgl. J.-M. ANDRÉ, Feste, 70.

${ }^{21}$ Vgl. J.-M. ANDRÉ, Feste, 211; Th. KLEMMER, Siegerkranz, 69 f.

${ }^{22}$ Vgl. W. O. MOELLER, The Riot of A. D. 59 at Pompeii, in: Historia 19, 1970, 84 ff. Zum Verbot, Alkohol mit ins Stadion zu bringen (bei empfindlicher Srafe) vgl. K. THOMAMÜLLER, Die Steininschrift am Stadion von Delphi. Vom überlieferten Text zum Original (Scripta redintegrata 3), Glückstadt 2007.

${ }^{23}$ Tac. Ann.15, 38 (Ausbruch eines Feuers im gefüllten Warengeschäft); CIL 6, 9822=ILS 7496 (pomarius)

${ }^{24}$ Suet. Aug. 74.

${ }^{25}$ Horat. Sat. 1, 6, 113 f.; J uv. 6, 588 ff.

${ }^{26}$ J uv. 3, 65: „ad circum iussas prostare puellas.“; Hist. Aug. Elagabal. 26, 3; 32. 9; Cyprian spectac. 5.

${ }^{27}$ Vgl.L. DREES, Olympia 75 f.;U. THESENVITZ, Olympia, 44 f. ; J .-M. ANDRÉ, Feste, 70.
} 
erwecken. ${ }^{28}$ Stets waren aber auch die großen Festveranstaltungen ein geeignetes Forum für politische Aktivitäten. Allein die hohen Zuschauerzahlen müssen für jeden ehrgeizigen Politiker und ruhmsüchtigen Kaiser von großem Interesse gewesen sein. Politische Ansprachen wurden schnell zur eigenen Wahlpropaganda und nahmen gelegentlich demagogische Züge an. Die Gelegenheit für eine wirkungsvolle Selbstdarstellung und Werbung in eigener Sache war für Regierende und Mächtige nirgendwo besser, als bei den stets gut besuchten Festveranstaltungen. Vor allem die römischen Kaiser nutzten die Spiele zur Demonstration ihrer persönlichen Macht wie auch der des Imperiums, u. a. durch die Darbietung exotischer Tiere aus aller Welt bei den munera. Ebenso konnte der Anspruch auf eine langfristige Regierungszeit durch Präsentation von Familie und designiertem Nachfolger Ausdruck verliehen werden. Der Medienspezialist Augustus ${ }^{29}$ setzte, was den Einsatz von Spielen aller Art zur Stabilisierung politischer Macht anbelangt, richtungsweisende Maßstäbe. Die Spiele waren besonders im kaiserlichen Rom zu einer absoluten Notwendigkeit geworden, was sehr deutlich J uvenals Worte von panem et circensis nachhaltig zum Ausdruck brachten. Durch die prunkvolle Gestaltung bestehender Feste oder die Stiftung neuer Veranstaltungen konnten Herrscher und führende Persönlichkeiten von Mängeln und Fehlern ablenken und sich so ein verherrlichendes Andenken an ihre Person schaffen. Häufig bestand bei neuen Regenten das Bedürfnis, ihre Vorgänger hinsichtlich Großzügigkeit zum Wohle und zur Unterhaltung des Volkes zu übertreffen. ${ }^{30}$ Dies konnte jedoch durch stetig steigende Kosten

${ }^{28}$ D. SANSONE, Genesis, 77: „Indeed, in the hands of the most distinguished poets and artists, sport becomes itself the subject of great art." Allgemein zum außersportlichen Treiben vgl.I. WEI LER, Olympia - jenseits der Agonistik:Kultur und Spektakel, in: Nikephoros 10, 1997, 193ff.

${ }^{29}$ Vgl. J.-M. ANDRÉ, Feste, 206.

30 M. CLAUSS, Kaiser und Gott, Herrscherkult im Römischen Reich, Stuttgart 1999, 334: „Sicherlich dienten die Ausrichtung von Gladiatorenspielen oder eine kostenlose Getreideverteilung in erster Linie dem Prestige des Spenders, der Stärkung ihrer - im 
zum Problem werden, zumal die ursprünglich vom Volk wohlwollend geschätzte Freigebigkeit sich zu einer Art Anspruch auf Spiele höchsten Niveaus gewandelt hatte. Auch verschiedene Versuche von Maßnahmen zur Kostendämpfung $^{31} \quad$ (z. B. Begrenzung der Fechterpaare bei Gladiatorenspielen) zeigten nur sehr begrenzt Wirkung. Selbst der den Spielen abgeneigt gegenüber stehende Stoiker Marc Aurel ${ }^{32}$ oder der knauserige Septimius Servus ${ }^{33}$ konnten die enormen Kosten für die prächtigen Volksbelustigungen nicht gänzlich eindämmen. Die öffentlichen Veranstaltungen hatten aber auch einen ambivalenten Charakter. Wagenrennen und munera (wie auch Theateraufführungen) boten bei der versammelten Volksmasse eine günstige Gelegenheit zur Meinungsäußerung. Die Anwesenheit führender und einflussreicher Persönlichkeiten nutzten die Zuschauer dazu, Forderungen ${ }^{34}$ und Beschwerden ${ }^{35}$, Bitten $^{36}$ und Wünsche ${ }^{37}$ auf unterschiedlichste Weise vorzutragen. Diese bezogen sich nicht nur auf die sportlichen Darbietungen, sondern konnten auch politische ${ }^{38}$, soziale oder private Aspekte zum Gegenstand haben. Man kannte auch Bewirtungen innerhalb der Sportstätten, -Sklaven verteilten erlesene Speisen im

weitesten Sinne - politischen Autorität. Hierbei gab es ein Wettbewerbsdenken, das es erschwerte, hinter die Leistungen seines Vorgängers zurückzufallen.“

${ }^{31} \mathrm{~J}$.-M. ANDRÉ, Feste, $220 \mathrm{ff}$.

${ }^{32}$ Hist. Aug. M. Aurel 17, 7; 23, 4; vgl.L. FRIEDLÄNDER, SG 2, 3.

${ }^{33}$ Herodian, 3, 8, 9 f.; vgl. FRIEDLÄNDER, SG 2, 3.

34 Joseph. B. J. 19, 25 (Mäßigung des Steuerdrucks); Plin. n. h. 34, 62 (Rückgabe der Lysippischen Statue).

${ }^{35}$ Cass. Dio 56, 1, 2 (wegen neuer strenger Ehegesetze); vgl. E. BALTRUSCH, Regimen, $74 \mathrm{f}$.

36 Begnadigung und Freilassung von zum Kampf mit Tieren Verurteilten; vgl.

L. FRIEDLÄNDER, SG 2, 6.

${ }^{37}$ Suet. Calig. 30, 2 (Auftreten berühmter Gladiatoren); Mart. Spectac. 29, 3 (Entlassung eines tapferen Fechters).

${ }^{38}$ Nach Dio Cass. 75,4,5 f. fand eine politische Demonstration besonderer Art im Jahre 196 n. Chr. Statt: Während zwischen Severus und dem Prätendenten Albinus der Bürgerkrieg tobte, forderte die Menge der Zuschauer im Circus einstimmig die Rückkehr zum Frieden. 
Zuschauerraum -, ${ }^{39}$ so wie auch Schenkungen durch im Zuschauerraum verteilte Lose, bei denen man Hausrat, Kleidung, Tiere, Grundstücke o. a. gewinnen konnte. ${ }^{40}$

Sportliche Veranstaltungen wurden von der ganzen Familie aufgesucht, soweit den Frauen die Anwesenheit nicht verwehrt war (z. B. in Olympia) ${ }^{41}$. Spannung und die erotische Ausstrahlung der Gladiatoren zogen auch die Frauen in ihren Bann. ${ }^{42}$ Selbst ein Teil der Kindes- und J ugenderziehung fand im öffentlichen Raum statt. Härte und Grausamkeit wurden öffentlich dargeboten: Der Strafvollzug fand z. T. öffentlich im Colosseum statt, was Genugtuung für die Opfer, Abschreckung für potentielle Straftäter, aber auch realistische Anschauung speziell für die Jugend hinsichtlich der zu erwartenden Strenge ihres zukünftigen (militärischen) Lebens sein sollte. ${ }^{43}$ Auch das ständig an Einfluss und Macht gewinnende Christentum ${ }^{44}$ brach nicht abrupt mit Sport und dem Spielwesen, sondern leistete lediglich einen Beitrag zu dessen stetigen Niedergang, der vor allem durch innen- und außenpolitische und ökonomische Umstände bewirkt wurde.

Man kann festhalten, dass Sport und sportliche Veranstaltungen sich allgemein grossem öffentlichem Interesse erfreuten und sehr gezielt von

\footnotetext{
${ }^{39}$ Suet. Calig. 18, 2; Domit. 4, 5.

${ }^{40}$ Suet. Nero, 11, 2; Cass. Dio 61, $1 \mathrm{f}$.

${ }^{41}$ W. DECKER, Sport, 106: „Es ist im übrigen merkwürdig, daß in Olympia verheiratete Frauen vom Zuschauen der Wettkämpfe ausgeschlossen waren, unverheiratete Frauen hingegen offenbar nicht."
}

${ }^{42}$ Vgl. S. MÜLLER, Volk, 252; D. G. KYLE, Spectacles. 318: „Not be overlooked or overemphasized, the erotic appeal of gladiators, like that of modern boxers and matadors, was related to the inherent psycho-sexual allure of violence. References to gladiators as sex symbols, however, come mostly from Ovid, Juvenal, and Martial, as well as graffiti at Pompeii."

${ }^{43}$ Th. WIEDEMANN, Adults and Children in the Roman Empire, London 1989. 27 und 62

${ }^{44} \mathrm{Vgl}$. A. KOCH, Die Leibesübungen im Urteil der antiken und frühchristlichen Anthropologie (=Beiträge zur Lehre und Forschung der Leibeserziehung 20), Schorndorf 1965, 57-134; DERS. In: GL 2, 312-340; W. WEI SMANN, Kirche und Schauspiele (=Cassiciacum 27), Würzburg 1972. 
privater wie besonders staatlicher Seite protegiert, reglementiert, und je nach Intention genutzt wurden. 


\section{2}

Professionalismus

Der Wettkampfsport in der griechisch-römischen Antike war von Anfang an ${ }^{1}$ eine ernst zu nehmende Tätigkeit ${ }^{2}$, die mit viel Aufwand und Mühen ${ }^{3}$ verbunden war. Die am Wettkampf beteiligten Sportler unterlagen keiner prinzipiellen Trennung in Amateure ${ }^{4}$ und Professionelle ${ }^{5}$. Zwar mag ein Sportler, der seine Zeit ausschließlich Training und Wettkampf widmete, einem weniger gut Vorbereiteten meist überlegen gewesen sein ${ }^{6}$, doch erwarteten beide Belohnung für ihre Leistung und empfanden untereinander keine Unterscheidung ${ }^{7}$.

Für den antiken Wettkampfsportler standen unabhängig von sozialem Status ${ }^{8}$ und/oder ethnischer Zugehörigkeit ${ }^{9}$ Leistung und Erfolg im Vordergrund ${ }^{10}$. Der Sieg, der erste Platz zählte, man wollte der Beste $\operatorname{sein}^{11}$. Diesem Streben nach größten sportlichen Erfolgen musste das persönliche Leben unterworfen ${ }^{12}$ und das gesamte Umfeld danach ausgerichtet werden ${ }^{13}$. Nur

${ }^{1}$ E. A. GLADER, Amateurism, 54: „From the time of Homer the athletics in ancient Greece were what is currently defined as ,professional'."

${ }^{2}$ Vgl. L. ROBERT, Lucillius, 196; M. POLIAKOFF, Kampfsport, 146.

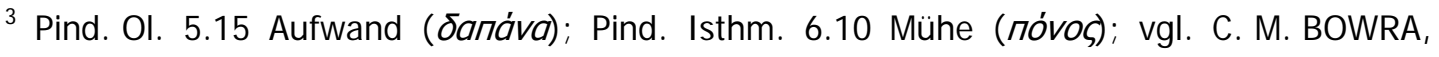
Pindar, 172; J. JÜTHNER, $A L$ 1, 88; R. MERKELBACH, in: ZPE 18, 1975, 110 f. u. 116.

${ }^{4}$ D.-C. YOUNG, in: Nikephoros 9, 1996, 178: "'Amateur athlete' was the one thing the Greeks had no word for."

${ }^{5}$ E. A. GLADER, Amateurism, 54: „In fact, the Greek did not make a distinction between professional and amateur athletes."

'Vgl. J. JÜTHNER, in: Die Leibesübungen 3, 1927, 117.

${ }^{7}$ Vgl. M. FINLEY/H. W. PLEKET, Games, 124.

${ }^{8}$ Vgl. G. HORSMANN, Wagenlenker, 77 ff., M. POLIAKOFF, Kampfsport, 179 ff.; D. G. KYLE, Athens, $102 \mathrm{ff}$.

${ }^{9}$ Vgl. G. VILLE, Gladiature, $227 \mathrm{ff}$.

${ }^{10}$ Vgl. P. FRISCH, Papyri, 7.

${ }^{11}$ Hom. II. 23; vgl. I. WEI LER, in: Stadion 2, 1975, 199 ff.

12 Vgl. M. POLIAKOFF, Kampfsport, 147; A. GUTTMANN, Ritual, 44: „There was a whole way of life concentrated on the single goal of athletic excellence."

${ }^{13}$ Vgl. H. BUHMANN, Sieg, 28. 
ein Athlet, der sich durch fleißiges, systematisches Training ${ }^{14}$ auf der Höhe seiner sportlichen Leistungsfähigkeit hielt, konnte dauerhaft mit Erfolgen bei den bedeutenden Wettkämpfen rechnen ${ }^{15}$.

Die professionelle Zusammenarbeit mit einem für den Sportler zuständigen Trainer war unerlässlich für das Erreichen einer maximalen Leistungsfähigkeit ${ }^{16}$. Diese gut bezahlten Trainer $^{17}$ wiederum waren häufig ehemalige Aktive $^{18}$, die auf eine langjährige und oft sehr erfolgreiche Karriere zurückblicken konnten ${ }^{19}$. I hnen oblag auch die Talentsichtung und die Einforderung von Mitteln zur Unterstützung des Erfolg versprechenden Nachwuchses. ${ }^{20}$ Allein aus ihrer eigenen Trainings- und Wettkampferfahrung konnten sie ihren Schützlingen große Dienste erweisen. Die Verbesserung von Physis ${ }^{21}$ und Technik ${ }^{22}$ stand im Vordergrund, aber auch die taktische ${ }^{23}$ und psychologische ${ }^{24}$ Schulung flossen in die Trainingsarbeit ein. Neben den eigenen Erfahrungen erweiterten die Trainer ihre Kenntnisse durch entsprechende Fachliteratur ${ }^{25}$ und ließen ihre Schüler nach

\footnotetext{
${ }^{14}$ Vgl. W. DECKER, Sport, 117, M. POLIAKOFF, Kampfsport, 24 ff.

15 Die jahrzehntelangen, äußerst erfolgreichen Laufbahnen eines Milon (sechsfacher Periodonike) oder eines Theogenes (1.300 Siege) sind sonst nicht zu erklären. Zu Porträts von den herausragenden Athleten mit Übersicht ihrer Leistungen vgl. W. DECKER, Sport, $107 \mathrm{ff}$;; im Besonderen zu Milon von Kroton $131 \mathrm{ff}$. und zu Theogenes von Thasos $133 \mathrm{ff}$.

${ }^{16}$ Vgl. J . JÜTHNER, AL 1, 93; W. DECKER, Sport, 117.

17 Nach W. RUDOLPH, Polis, 1482 n. 8, lag das Gehalt eines Trainers per anno um 300 v. Chr. bei 500 Drachmen.

${ }^{18}$ Vgl. J. JÜTHNER, $A L$ 1, 85; W. DECKER, Sport, 118.

${ }^{19}$ Vgl. J. JÜTHNER, $A L$ 1, 26; W. DECKER, Sport, 123 ff.

${ }^{20}$ L. ROBERT, in: RPh 41, 1967, 7 ff.; H. W. PLEKET, in: MNIR 36, 1974, 69 f.; S. MÜLLER, Volk, $29 \mathrm{f}$.

${ }^{21}$ Vgl. W. DECKER, Sport, 121 ff., M. POLIAKOFF, Kampfsport, 29.

${ }^{22}$ Vgl. I. WEI LER, Sport, 178.

${ }^{23}$ Vgl. S. MENDNER, Ballspiel, 81; W. DECKER, Sport, 27.

${ }^{24}$ Vgl. R. PATRUCCO, La psicologia dell'atleta, in: Maia 23, 1971, 245 ff., A. GUTTMANN, Ritual, 43; W. DECKER, Sport, 145.

${ }^{25}$ Vgl. J. JÜTHNER, $A L$ 1, 21 ff.; W. DECKER, Sport, 117 f.
} 
wissenschaftlichen Methoden trainieren ${ }^{26}$. Berühmt war ein viertägiges Intervalltraining, bei dem der erste Tag kurz dauernde, hohe Belastungen, der zweite Maximalanstrengungen mit Simulierung der Wettkampfsituation, der dritte ein Entspannungstraining und der vierte Tag schließlich mittlere Belastungen bringen sollte. ${ }^{27}$

Die Trainingsformen konnten sich auf unterschiedliche Art und Weise gestalten: Einzelübungen, die vor allem der physischen Leistungssteigerung bezüglich Beweglichkeit, Kondition und Kraft dienen sollten, waren in erster Linie Gymnastik, Läufe und Training mit Gewichten. ${ }^{28}$ In der Schwerathletik kannte man zur Technikschulung beim Faustkampf das Schattenboxen

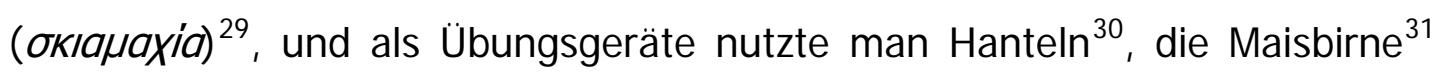
und den Sandsack ( $\kappa \dot{\rho} \rho \kappa \kappa \varsigma$ ), der auch von den Pankratiasten zum Training ihrer Beintechniken diente. ${ }^{32}$ Die Gladiatoren übten mit besonders schweren Holzschwertern ${ }^{33}$ und schulten ihre Technik am Übungspfahl (palus) ${ }^{34}$. Der größte Teil des Trainings bei den Schwerathleten wurde in Form von Partnerübungen wie etwa bei Boxern und Pankratiasten mit leichtem Sparring $^{35}$ durchgeführt, z. B. bei den Boxern mit Ohrenschutz

\footnotetext{
${ }^{26}$ Vgl. W. DECKER, Sport, 119.

${ }^{27}$ Vgl. W. DECKER, Sport, 121; A. GUTTMANN, Ritual, 43 f; G. LUKAS, Körperkultur, 110: „Auf diese Weise wurden neue Wege erschlossen, um sportliche Höchstleistungen zu erreichen."
}

${ }^{28}$ Vgl. W. E. SWEET, Sport, 106, Abb. 37, W. DECKER, Sport, 122.

${ }^{29}$ Vgl. I. WEI LER, Sport, 178; M. POLIAKOFF, Kampfsport, 28 u. $117 \mathrm{f}$.

30 Vgl. W. DECKER/J .-P. THUILLIER, Sport, 244, Fig. 139.

${ }^{31} \mathrm{Vgl}$. R. PATRUCCO, Sport, 265, Abb. 119; W. BACKHAUS, GL 2, 148, Abb. 36; M. POLIAKOFF, Kampfsport, 30: „Die Faustkämpfer benutzten kleine Kornsäcke ähnlich den von heutigen Boxern gebrauchten Maisbirnen. “ Vgl. 29, Abb. 5.

${ }^{32}$ Vgl. I. WEILER, Sport, 178; M. POLIAKOFF, Kampfsport, 26 u. 29 f.: ,... während die Pankratiasten ihre Tritte und Schläge an viel größeren Säcken übten, die anstatt mit Korn mit Sand gefüllt waren.", W. DECKER, Sport, 121.

${ }^{33} \mathrm{Vgl.} \mathrm{M.} \mathrm{JUNKELMANN,} \mathrm{Spiel,} \mathrm{21;} \mathrm{D.} \mathrm{RAMBA,} \mathrm{Gladiatur,} 54$.

${ }^{34}$ Vgl. M. JUNKELMANN, Spiel, 21, Abb. 21.

${ }^{35}$ Vgl. M. POLIAKOFF, Kampfsport, 28. 
$(a ́ \mu \varphi \omega t i \delta \varepsilon \varsigma),{ }^{36}$ wobei Sportler derselben Disziplin unter der Aufsicht der Trainer miteinander übten. ${ }^{37}$ Beim Training der Gladiatoren konnten Kämpfer derselben, wie auch unterschiedlicher armatura miteinander üben oder wurden von speziellen doctores - jede Kampfgattung kannte einen speziellen Trainer - individuell geschult. ${ }^{38}$

Auch die Ernährung ${ }^{39}$ war auf die speziellen Bedürfnisse der Sportler abgestimmt. ${ }^{40}$ In der Athletik ernährten sich Schwerathleten anders als Leichtathleten, ${ }^{41}$ und innerhalb dieser Kategorien gab es Unterschiede, was deutlich wird beim Anblick von Statur und Muskelaufbau von Kurzstreckenbzw. Langstreckenläufern. ${ }^{42}$ Bei der Schwerathletik galt ein großer, kräftiger und muskulöser Körper von Vorteil, da es keine Einteilung in Gewichtsklassen gab. ${ }^{43}$ Dieser Grundsatz fand seine Grenzen, wenn dadurch die Beweglichkeit zu sehr eingeschränkt wurde. ${ }^{44}$ Eine besonders feine Abstimmung des Speiseplans fand sich bei den Gladiatoren, da deren physischer Zustand der Waffengattung entsprechend von existentieller Bedeutung war. ${ }^{45}$ Für die Mehrzahl der Spitzensportler gab es auch eine sehr gute und kompetente medizinische ${ }^{46}$ sowie physiotherapeutische ${ }^{47}$ Betreuung in Anlagen, die durch Klima und Lage das Wohlbefinden der Athleten steigerte und für ein

\footnotetext{
${ }^{36}$ Vgl. M. POLIAKOFF, Kampfsport, 27, Abb. 4 (beim Training).

${ }^{37}$ Vgl. M. POLIAKOFF, Kampfsport, 24 ff.; I. WEI LER, Sport, 178.

${ }^{38}$ Vgl. M. J UNKELMANN, Spiel, 73.

${ }^{39} \mathrm{Vgl}$. W. RUDOLPH, Kampfsport, 44 f.; K. GROSSSCHMIDT/F. KANZ (Hrsg.), Ephesos, 37 f.

${ }^{40}$ Vgl. I. WEILER, Sport, 172.

${ }^{41}$ W. DECKER, Sport, 70.

${ }^{42}$ Vgl. M. BENTZ, Panathenäische Preisamphoren, Basel 1998, 9 ff.

${ }^{43}$ Vgl. I. WEILER, Sport, 172.

${ }^{44}$ Vgl. I. WEILER, Sport, 172.

${ }^{45}$ Vgl. M. JUNKELMANN, Panis Militaris - Die Ernährung der römischen Soldaten oder der Grundstoff der Macht, Mainz 2006, 117 f.

${ }^{46}$ So etwa der Gladiatorenarzt und zeitweilige kaiserlicher Leibarıt Galenos von Pergamon.

${ }^{47}$ Vgl. D. RAMBA, Gladiatur, 54; M. JUNKELMANN, Spiel, 78.
} 
attraktives Äußeres förderlich waren. ${ }^{48}$ Auch sexuelle Enthaltsamkeit galt als leistungsfördernd. ${ }^{49}$

Große Anerkennung und hoher Bekanntheitsgrad eines Erfolges bei den bedeutenden Spielen - in zahlreichen Epinikien verherrlicht ${ }^{50}$ - galten nicht nur dem siegreichen Sportler, sondern auch seinem Trainer $^{51}$ und seiner Familie. ${ }^{52}$ Auch die Heimatgemeinde ${ }^{53}$ und gelegentlich eine ganze Region ${ }^{54}$ - z. B. hinsichtlich der Pferdezucht ${ }^{55}$ - konnte den Ruhm genießen und wirtschaftlich davon profitieren. ${ }^{56}$ Den größten Vorteil eines sportlichen Triumphes hatte selbstverständlich der Sportler selbst. Ihm winkten zum Teil beträchtliche finanzielle Gewinne ${ }^{57}$ und ansehnliche Sachpreise ${ }^{58}$. Man denke nur an die von Solon ${ }^{59}$ ausgesetzten Siegprämien oder die beachtliche Zahl an Panathenäischen Preisamphoren ${ }^{60}$ samt des begehrten Olivenöls, an die Prämien für erfolgreiche Gladiatoren ${ }^{61}$ und venatoren ${ }^{62}$ sowie die enormen Gewinnsummen der römischen Wagenlenker. ${ }^{63}$

${ }^{48}$ Vgl. M. JUNKELMANN, Spiele, 152.

${ }^{49}$ Vgl. H. BUHMANN, Sieg, 36.

${ }^{50}$ Vgl. G. STEINKOPF, Untersuchungen, 53 ff.; L. ROBERT, in: RPh 41, 1967, 18 ff.

${ }^{51}$ Vgl. H. BUHMANN, Sieg, 31 f.; G. LUKAS, Körperkultur, 110, weist darauf hin, dass die Trainer ihre Athleten stets zu den Wettkämpfen begleiteten.

${ }^{52} \mathrm{Vgl}$. L. DREES, Olympia, $116 \mathrm{ff}$.

${ }^{53}$ Vgl. J. EBERT, Epigramme, 10 f.; U. THESENVITZ, Olympia, 113.

${ }^{54}$ Thessalien war bekannt für seine hervorragenden Rennpferde.

${ }^{55} \mathrm{Vgl} \mathrm{U}$. THESENVITZ, Olympia, 113.

${ }^{56}$ Vgl. P. VIGNERON, Sport, 145

${ }^{57}$ Vgl. zur Umrechnung in aktuelle Währung (Dollar) D. C. YOUNG, Myth, 124 ff.

${ }^{58}$ Vgl. Th. KLEE, Geschichte, 20 ff.; D. C. YOUNG, Myth, $113 \mathrm{f.}$

${ }^{59}$ Vgl. H. BUHMANN, Sieg, 106.

${ }^{60}$ ggl. D. C. YOUNG, Myth, $121 \mathrm{ff}$.

${ }^{61}$ Vgl. G. VILLE, Gladiature, $426 \mathrm{f}$.

${ }^{62}$ Vgl. V. OLIVOVÁ, Sport, 190, Abbildung eines Mosaiks aus Nordafrika, auf dem ein Arenadiener dargestellt ist, der Beutel voll Geld für erfolgreiche venatrores zur Schau stellt.

${ }^{63} \mathrm{Vgl}$. A. CAMERON, Factiones, 62; D. RAMBA, Verdienstmöglichkeiten von Spitzensportlern in der Antike, in: Sozial- und Zeitgeschichte des Sports 4, 1990, $75 \mathrm{f}$. 
Auch sozialer Aufstieg ${ }^{64}$ und eine Anzahl von Vergünstigungen und Annehmlichkeiten seitens der Heimatgemeinde, wohlhabender Förderer oder der politischen Führung konnten mit dem herausragenden sportlichen Erfolg verknüpft sein: feierlicher Empfang ${ }^{65}$, Aufstellung einer Statue ${ }^{66}$, freie Speisungen im Prythaneion ${ }^{67}$, Ehrenmitgliedschaft im Rat ${ }^{68}$ u. v. m.. Die erfolgreichen Sportler wurden zu Berühmtheiten mit entsprechendem Starkult ${ }^{69}$ und konnten zum Objekt der Begierde werden. So ist es nicht verwunderlich, dass infolge des Wunsches nach Ruhm und Anerkennung einflussreiche Personen oder vornehme Geschlechter viel - aber auch unlautere Mittel $^{70}$ - daran setzten, sportliche Erfolge bzw. die Sieger/Titelträger für sich vereinnahmen zu können ${ }^{71}$. Angesichts dieser zahlreichen finanziellen und materiellen Anreize und den mit Siegen und Titeln verbundenen Ehrungen und Privilegien kam es im Wettkampfwesen zu Manipulationen und betrügerischen Absprachen ${ }^{72}$. Die aus Strafgeldern errichteten Statuen (Zanes) in Olympia sind hierfür der anschauliche Beweis $^{73}$.

${ }^{64}$ Vgl. H. W. PLEKET, Zur Sozilogie des antiken Sports, in: MNIR 36, 1974, 57 ff.

${ }^{65}$ Vgl. H. BUHMANN, Sieg, 104 f.; F. BOEHRINGER, in: REA 81, 1979, 5-18.

${ }^{66}$ Vgl. H. BUHMANN, Sieg, 107 ff.; F. RAUSA, L'immagine del vincitore, Treviso/Rom 1994, (z. B. Milon von Kroton), $82 \mathrm{f}$.

${ }^{67}$ Vgl. H. BUHMANN, Sieg, $111 \mathrm{f.;}$ vgl. zu einer Auflistung zahlreicher Privilegien M. AMELOTTI, in: SDHI 21, 1955, 123ff.

${ }^{68} \mathrm{Vgl}$. H. BUHMANN, Sieg, $113 \mathrm{f}$.; Bisweilen ist manch berühmter Athlet von mehreren

Poleis mit Ehrenbürgerrecht und Ehrenmitgliedschaft im Rat ausgezeichnet wurden.

H. W PLEKET, Games, Prizes, Athletes and Ideology, in: Stadion 1, 1975, 49-89.

${ }^{69}$ Zur Heroisierung einiger Olympiasieger vgl. H. BUHMANN, Sieg, 134 ff.; S. MÜLLER, Volk, 172 u. $181 \mathrm{f}$.

${ }^{70}$ Vgl. J. JÜTHNER, $A L$ 1, 149; W. RUDOLPH, in: Poleis, 1474.

${ }^{71}$ Vgl. W. DECKER, Sport, 125.

${ }^{72}$ Vgl. C. A. FORBES, Crime and Punishment in Greek Athletics, in: CJ 47, 1952, 169 ff. u. 202 f.; H. BUHMANN, Sieg, 45 ff.; W. E. SWEET, Sport, 223 f.

${ }^{73}$ Vgl. I. WEILER, Sport, 125; W. DECKER, Sport, 127; W. E. SWEET, Sport, 239 f. 
Die international agierenden ${ }^{74}$ Wettkampfsportler waren in genossenschaftlichen Verbänden organisiert ${ }^{75}$, die sich um die Ausrichtung der Veranstaltungen kümmerten ${ }^{76}$, stets um ein gutes Verhältnis zu den Herrschenden (z. B. in Form der Pflege des Kaiserkultes) bemüht waren ${ }^{77}$, hauptsächlich aber die Interessen ihrer Mitglieder wahrnahmen ${ }^{78}$. Die Sportler selbst pflegten einen kameradschaftlichen Umgang miteinander, ${ }^{79}$ trainierten zusammen und bestritten oft gemeinsam ihre Wettkampftournee ${ }^{80}$. Bei allem Ehrgeiz und den Aussichten auf beträchtliche Gewinne hatte das Prinzip des Fair Play einen gewissen Stellenwert. ${ }^{81}$

\footnotetext{
${ }^{74}$ Vgl. J. JÜTHNER, $A L$ 1, 145; I. WEILER, Sport, 97.

${ }^{75} \mathrm{Vgl}$. C. A. FORBES, in: CPh 50, 1955, $238 \mathrm{ff}$; B. BILINSKI, L'agonistica, $57 \mathrm{ff} .$, W. DECKER/J .-P. THUI LLIER, Sport, 123; H. W. PLEKET, Some Aspects oft he History of the Athletic Guilds, in: ZPE 10, 1973, 197.

${ }^{76}$ Vgl. M. L. CALDELLI, Curia athletarum, hiera xystike synodos e organizzazione delle terme a Roma, in: ZPE 93, 1992, 75 ff.; J.-M. ANDRÉ, Feste, 51; U. SI NN, Olympia, 99 f.

${ }^{77}$ Vgl. H. AlGNER, in: Civitas 40, 1985, 298; LE GUEN, B: Hadrien, I'Empereur philhellène, et la vie agonistique de son temps: Hadrian und die dionysischen Künstler. Drei in Alexandreia Troas neugefundene Briefe des Kaisers an die Künstler-Vereinigung, in: Nikephoros 23, 2010, 205-239.

${ }^{78} \mathrm{Vgl}$. M. AMELOTTI, La posizione degli atleti di fronte al diritto romano, in: SDHI 21, 1955, $123 \mathrm{ff}$.

${ }^{79}$ So sprachen die Gladiatoren untereinander von coarmio und convictor.

${ }^{80}$ Vgl. W. DECKER/J.-P. THUILLIER, Sport, 123, Fig. 74, mit den Stätten der Siege des Pankratiasten M. Aurelius Asklepiades aus Alexandria Ende des 2. J hd. n. Chr.

${ }^{81}$ Vgl. H. W. PLEKET, in: MNIR 36, 1974, 57 ff; A. BRIERS, Sporting Success in Ancient Greece and Rome, Oxford 1994, $14 \mathrm{f}$.
} 


\subsection{Chancen}

Für Griechen und Römer war die Gleichheit der Wettkampfumstände eine äußerst wichtige Angelegenheit, und sie gaben sich größte Mühe, diese herzustellen ${ }^{1}$. Für alle Sportler galten dieselben Formalitäten und Regeln. ${ }^{2}$ Jeder freie männliche, unbescholtene Grieche ${ }^{3}$ konnte - unabhängig von seinem sozialen Status ${ }^{4}$ - den Siegerpreis erhalten, wenn er gewann, denn das Einzige, was zählte, waren sein eigenes Geschick und seine Leistung. ${ }^{5}$

Die meisten der Maßnahmen und Einrichtungen zur Herstellung von Chancengleichheit galten für alle Wettkämpfe und nur einige für spezielle Sportarten und -disziplinen bzw. deren Austragungsstätten. ${ }^{6}$

Schon im Vorfeld einer Veranstaltung wurde für chancengleiche Wettkampfbedingungen gesorgt. So mussten z. B. die Teilnehmer an den Olympischen Spielen beeiden, dass sie sich zehn Monate lang auf die Wettkämpfe vorbereitet hatten ${ }^{7}$ und mussten sich vor Ort unter Aufsicht der Hellanodiken einem dreißigtägigen Training unterwerfen ${ }^{8}$.

${ }^{1}$ A. GUTTMANN, Ritual, 28: „Equality of the conditions of competition was important to the Greeks and they went to considerable trouble to avoid certain inequality."

${ }^{2}$ Vgl. M. I. FINLEY/H. W. PLEKET, Spiele, 58.

${ }^{3}$ Vgl. W. DECKER, Sport, 40; Th. KLEMMER, Siegerkranz, 55ff.

${ }^{4}$ Vgl. A. GUTTMANN, Ritual, 28; vgl. N. B. CROWTHER, Slaves and Greek Athletics, in: QUCC 40.1, 1992, $39 \mathrm{ff}$. , wonach Sklaven in der griechischen Welt nicht von allen sportlichen Veranstaltungen ausgeschlossen waren.

${ }^{5}$ Vgl. M. I. FINLEY/H. W. PLEKET, Spiele, 58; A. GUTTMANN, Ritual, 28.

${ }^{\complement}$ Vgl. M. I. FINLEY/H. W. PLEKET, Spiele, 56.

7 Paus. 5, 24, 9; vgl. H. BUHMANN, Sieg, 31; J.-M. ANDRÉ, Feste, 69; H. M. LEE, The Program and Schedule of the Ancient Olympic Games, Hildesheim 2001, (=Nikephoros Beihefte, Bd. 6), 26; U. SI NN, Olympia, 77 f;Th. KLEMMER, Siegerkranz, 56.

${ }^{8}$ Paus. 6, 23, 1 f. ; vgl. H. BUHMANN, Sieg, 31; W. RUDOLPH, Kampfsport, 2; J.-M. ANDRÉ, Feste, 69; N. B. CROWTHER, The Olympic Training Period, in: Nikephoros 4, 1991, 161 ff. (=Athletika 65 ff.); U. SINN, Olympia, 58: „Der gemeinsame Aufenthalt in Elis ließ schließlich 
Das Teilnehmerfeld an den athletischen Wettkämpfen unterlag einer Unterteilung in Altersklassen, nicht aber in Gewichtsklassen. ${ }^{9}$ Bei den Olympien und Pythien war das gesamte Starterfeld in nur zwei Altersklassen,

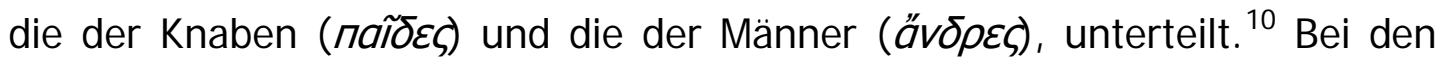

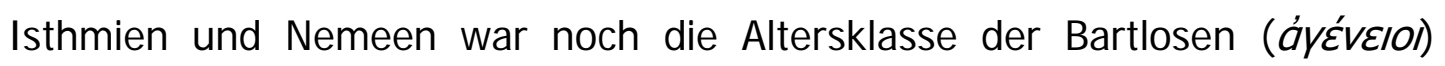
vertreten, und diese Dreiteilung galt auch für die meisten anderen Veranstaltungsorte. ${ }^{11}$ Wir wissen aber auch von Festen, bei denen die Teilnehmer einer noch detaillierteren Gliederung unterlagen. ${ }^{12}$ Für die hippischen Agone galt die Unterteilung in erwachsene Pferde, Fohlen und Maultiere. ${ }^{13}$ Die Altersklassenzuordnung aller Wettkampfteilnehmer oblag den Kampfrichtern. ${ }^{14}$ Die Entscheidung wurde durch Augenschein vollzogen, was zur Folge haben konnte, dass aufgrund ihrer Physis Teilnehmer einer anderen Klasse zugeschlagen wurden, die von ihrem Lebensalter abwich, ${ }^{15}$

auch eine Kontrolle der Nahrung zu,so daß geheime Rezepturen für spezifische Kraftnahrung (Doping) nicht zur Anwendung kommen konnten.“

'Vgl. J.-M. ANDRÉ, Feste, 120; A. GUTTMANN, Ritual, 28.

${ }^{10}$ Vgl. Th. KLEE, Geschichte, 46 ff.; I. WEI LER, Sport, 112; W. DECKER, Sport, 39.

${ }^{11}$ Vgl. J. JÜTHNER, $A L$ 2, 97; I. WEILER, Sport, 132.

${ }^{12} \mathrm{Vgl}$. J. EBERT, Zu Fackelläufen und anderen Problemen in einer griechischen agonistischen Inschrift aus Ägypten, in: Stadion 5, 1979, 15 f.; P. FRISCH, Die Klassifikation der naĩdes bei den griechischen Agonen, in: ZPE 75, 1988, 179 f.; L. KOENEN, Eine agonistische Inschrift aus Ägypten und frühptolemäische Königsfeste, Hildesheim 1977 (=Beiträge zur klassischen Philologie; H. 56), $15 \mathrm{ff.}$

${ }^{13}$ Vgl. I. WEI LER, Sport, 205; W. DECKER, Sport, 109, weist darauf hin, dass die Disziplinen Wagenrennen und Reiten bis zum Beginn des 4. J hs. V. Chr. ohne Einteilung in Altersklassen blieben.

${ }^{14}$ Vgl. J. JÜTHNER, $A L$ 2, 37; W. DECKER, Sport, 101, Abb. 50 mit dem Stimmtäfelchen eines Kampfrichters bezüglich der Altersklasseneinteilung.

${ }^{15}$ Vgl. H. BUHMANN, Sieg, 42; A. GUTTMANN, Ritual, 28; P. FRISCH, in: ZPE 75, 1988, 179 ff.; N. B. CROWTHER, in: Phoenix 42, 1988, 307: „However, in cases of doubt or dispute it is evident that size could supersede age." . 
oder aufgrund ihres zu jungen Alters nicht zu den Knabenwettkämpfen zugelassen wurden. ${ }^{16}$

Nach der Einteilung in Altersklassen erfolgte die Auslosung zur Schaffung größtmöglicher Chancengleichheit. ${ }^{17}$ Auch in der Schwerathletik wurden die Kampfpaare ohne Vorbehalt ausgelost. ${ }^{18}$ Bei einer ungeraden Zahl an Teilnehmern gab es Freilose ${ }^{19}$, was von Vorteil sein konnte. Wir wissen von Finalsiegern, dass sie, um ihren Erfolg aufzuwerten, anführten, ohne Freilos - vielleicht sogar noch gegen einen Gegner, der zuvor pausieren durfte - das Finale gewonnen hatten, was der Leistung einen höheren Stellenwert verlieh. ${ }^{20}$ Bei den Laufkonkurrenzen ${ }^{21}$, den hippischen Agonen $^{22}$ und Wagenrennen ${ }^{23}$ wurden durch das Los die Vorrundenzusammenstellung bzw. die Startposition bestimmt.

Der gesamte antike Wettkampfsport wurde durch ein genau vorgegebenes Regelwerk bestimmt. ${ }^{24}$ Obwohl uns einschlägige schriftliche Quellen zum Regelwerk nur recht spärlich zur Verfügung stehen, ${ }^{25}$ lassen sich vor allem anhand der bildlichen Darstellungen bzw. den Aktionen der Kampfrichter die ${ }^{16}$ Paus. 6, 14, 1; vgl. M. I. FI NLEY/H. W. PLEKET, Spiele, 114.

${ }^{17} \mathrm{Vgl}$. N. B. CROWTHER, Rounds and Byes in Greek Athletics, in: Stadion 18, 1992, $68 \mathrm{ff}$. (=Athletika $215 \mathrm{ff}$. ).

18 Da wir nur in Ausnahmefällen den unterlegenen Finalisten kennen, bleibt es offen, inwieweit Mitfavoriten schon in den Vorrunden ausgeschieden sind, oder ein potentielles Finale in der ersten Runde stattfand.

19 Zum Freilos ( $\left.\varepsilon^{\prime} \varphi \varepsilon \delta \rho o \zeta\right)$ vgl. I. WEILER, Sport, 175 (Ringkampf), 179 (Boxen), 185 (Pankration).

${ }^{20}$ Vgl. H. BUHMANN, Sieg, 43.

${ }^{21}$ Zur Zahl der Startplätze vgl. S. G. MILLER, Turns and Lanes in the Ancient Stadium, in: AJ A 84, 1980, $159 \mathrm{ff}$.

${ }^{22} \mathrm{Vgl}$. J. EBERT, Agonismata, $37 \mathrm{ff}$.

${ }^{23} \mathrm{Vgl}$. J. H. HUMPHREY, Circuses, $132 \mathrm{ff}$.

${ }^{24} \mathrm{Vgl}$. M. B. POLIAKOFF, Kampfsport, $44 \mathrm{ff}$.

${ }^{25} \mathrm{Vgl}$. A. GUTTMANN, Ritual, 42: „Despite the best efforts of classical scholars, we still know less than we would like about the rules of Greek and Roman sports.“ 
erlaubten bzw. regelwidrigen Aktionen erschließen. ${ }^{26}$ Ferner kennen wir die Gründe, die zur Disqualifikation sowohl in Bereichen der Schwer- und Leichtathletik als auch im Pferdesport geführt haben. ${ }^{27}$ Wohl werden die Wettkampfbestimmungen von Olympia - von einigen wenigen lokalen Sonderregelungen abgesehen - für die meisten anderen Veranstaltungen maßgebend gewesen sein. ${ }^{28}$

Für die Leitung der Kämpfe und die Kontrolle über die Einhaltung der Wettkampfregeln waren gut geschulte und hoch angesehene Schiedsrichter zuständig. ${ }^{29}$ Mit großer Autorität ausgestattet konnten sie unfaire Sportler unabhängig von deren sozialem Status auf der Stelle züchtigen, was auf einer Vielzahl von Vasenbildern dargestellt ist. ${ }^{30}$

Die Nacktheit der Athleten war spätestens seit der Zeit der Perserkriege zum Standard geworden. ${ }^{31}$ Zur Begründung für die unbekleidete Wettkampfausübung bei der Leicht- und Schwerathletik finden sich bei antiken und modernen Autoren unterschiedliche Überlegungen und Angaben $^{32}$. So verstand man die Nacktheit in Anlehnung an den Kultus, ${ }^{33}$ sah

\footnotetext{
${ }^{26}$ Auf zahlreichen Vasenbildern sieht man das entschlossene Einschreiten von Kampfrichtern bei regelwidrigen Aktionen der Wettkämpfer.

${ }^{27}$ Vgl. C. A. FORBES, Crime and Punishment in Greek Athletics, in: CJ 47, 1951-1952, 169 ff. u. 202.
}

${ }^{28} \mathrm{Vgl}$. W. RUDOLPH, Kampfsport, 2.

${ }^{29}$ Vgl. J. JÜTHNER, $A L$ 1, $170 \mathrm{ff}$.

${ }^{30}$ Vgl. N. B. CROWTHER, Flogging as a Punishment in the Ancient Games, in: Nikephoros 11, 1998, $51 \mathrm{ff}$.

${ }^{31}$ N. B. CROWTHER, Athletic Dress and Nudity in Greek Athletics, in: Eranos 80, 1982, 163 : ,... nudity in athletics may have become a permanent feature in Greek athletics after the Persian Wars."

${ }^{32}$ Vgl. I WEILER, Sport, 109 u. $116 \mathrm{f}$.

${ }^{33}$ Vgl. R. HARDER, Eigenart, 13 ff., H. BENGTSON, Sport, 59; N. SPIVEY, Understanding Greek Sculpture: Ancient Meanings, Modern Readings, New York1996, 111 ff. 
darin den Ausdruck körperlicher (sexueller) Selbstkontrolle ${ }^{34}$ oder den Wunsch nach Zurschaustellung bzw. Anblick schön gestalteter Körper ${ }^{35}$ bei gleichzeitiger Abgrenzung zu den Barbaren. ${ }^{36}$ Der Überlieferung nach war es der Läufer Orsippus von Megara ${ }^{37}$, der bei den Olympischen Spielen 720 v. Chr. entgegen dem Brauch seinen Lendenschurz (perizoma) ${ }^{38}$ absichtlich fallen ließ, um schneller zu sein, ${ }^{39}$ was inm letztendlich den Sieg brachte. ${ }^{40}$ Dieses Ereignis mag ausschlaggebend gewesen sein, dass zur Schaffung von Chancengleichheit für alle Athleten das nackte Auftreten angeordnet wurde.

Was die besondere Wettkampfvorbereitung, die Sportgeräte bzw. die Rüstung und Bewaffnung anbelangt, finden wir auch hier stets das Bemühen um gleiche Chancen für alle Teilnehmer. Ein auffälliges Beispiel bietet uns der Ringkampf, der ohne Geräte oder Hilfsmittel ausgeführt wurde. Bei der unmittelbaren Wettkampfvorbereitung, bei der sich die Athleten mit Olivenöl eingerieben hatten, bewarf jeder Ringer seinen Gegner mit feinem Sand, damit garantiert war, dass keiner durch das Einölen einen Vorteil haben könnte. ${ }^{41}$ Beim Boxkampf, der mit einer Faustwehr ausgetragen wurde, ist

\footnotetext{
${ }^{34}$ Vgl. J. A. ARIETI, Nudity in Greek Athletics, in: CW 68, 1975, 431 ff.; zur homoerotischen Komponente der Nacktheit vgl. Ch. MANN, Griechischer Sport und griechische Identität: die certamina athletarum in Rom, in: Nikephoros 15, 2002, $147 \mathrm{f}$.

35 Luk. Anarch. 36; vgl. N. B. CROWTHER, in: Eranos 80, 1982, 140: ,... the Greeks were proud of their bodies, their lack of flab, their tan. " Vgl. auch C. H. HALLEIT, The Roman Nude. Heroic Portrait Statuary 200 BC - AD 300, Oxford 2005, bes. 68-87.

${ }^{36}$ Vgl. J. A. ARIETI, CW 68, 1975, 436; N. B. CROWTHER, in: Eranos 80, 1982, 140: "It was nudity, which separated them from barbarians, from whom they wished to be distinguished." ${ }^{37}$ Auch die Schreibung Orrhippus von Megara ist geläufig.

${ }^{38}$ Vgl. M. POLIAKOFF, Kampfsport, 89, Abb. 62 (Pankratiasten)

${ }^{39}$ Vgl. I. WEILER, Sport, 116.

${ }^{40}$ Paus. 1, 44, 1.

${ }^{41}$ Vgl. C. ULF, Die Einreibung der griechischen Athleten mit Öl, in: Stadion 5, 1979, 220 ff.; W. SWEET, Sport, 66: „The wrestlers rubbed themselves with olive oil and then, just before their match, covered each other with dust to furnish a good grip. ", Abb. 20.
} 
im Laufe der Jahrhunderte ein Wandel des Sportgerätes festzustellen. ${ }^{42}$ Bei bildlichen Darstellungen von Boxkampfszenen sehen wir aber, dass die Kontrahenten stets dieselbe manica verwendeten. ${ }^{43}$ Auch die unrühmliche Verwendung der mit Metallstücken versehenen Faustwehr (caestus) ${ }^{44}$ war nur in einer relativ kurzen Phase des Boxsportes bekannt, wobei stets von beiden Kontrahenten dieselbe Faustwehr benutzt wurde. ${ }^{45}$ Das Pankration wurde ohne Sportgerät oder spezielle Vorbereitung durchgeführt, galt als extrem harte und sehr beliebte Kampfsportart, bei der fast alle Techniken erlaubt waren, ${ }^{46}$ unterlag aber dennoch einem strengen Regelwerk, was zahlreiche Darstellungen belegen, bei denen Aktive nach Regelverstößen gezüchtigt wurden. ${ }^{47}$

Für die Laufkonkurrenzen - zumindest was die Kurzstrecken anbelangt nutzte man ein geschickt konstruiertes System von Ablaufschranken, ${ }^{48}$ deren Zweck vor allem darin bestand, Frühstarts zu verhindern, und somit allen Teilnehmern gleiche Chancen zu geben. ${ }^{49}$ Über den Waffenlauf wissen wir von einer Anzahl standardisierter Schilde, die den Wettkämpfern zur Verfügung gestellt wurden. ${ }^{50}$

\footnotetext{
${ }^{42}$ Vgl. S. MENDNER, Boxhandschuhe im Altertum, in: Gymnasium 60, 1953, 20 ff.

${ }^{43} \mathrm{Vgl}$. V. OLIVOVÁ, Sport, 115.

${ }^{44} \mathrm{Vgl}$. H. M. LEE, The Later Greek Boxing Glove and the "Roman" caestus: a Centennial Reevaluation of J üthner's ,Über Antike Turngeräte', in: Nikephoros 10, 1997, 161 ff..

${ }^{45} \mathrm{Vgl}$. G. LUKAS, Sport, 122: „Man achtete sogar darauf, daß beide Kämpfer den gleichen Cästus trugen, um Chancengleichheit zu bewahren.“

${ }^{46} \mathrm{Vgl}$. W. E. SWEET, Protection of the Genitals in Greek Athletics, in: AncW 11, 1985, $43 \mathrm{ff}$.

${ }^{47}$ VGI. M. B. POLIAKOFF, Kampfsport, 81, Abb. 53.

${ }^{48}$ Vgl. H. A. HARRIS, SGR, 27 ff.; I. WEILER, Sport, $132 \mathrm{f}$.

${ }^{49}$ Vgl. W. DECKER, Sport, 46: „Die Funktion des Startmechanismus, ... , den Athleten objektive Wettkampfbedingungen zu schaffen und Chancengleichheit herzustellen. “

${ }^{50}$ Paus. 5, 17, 6; vgl.W. DECKER, Sport, 61: „Aus Gründen der Chancengleichheit waren die Schilde genormt: 25 von innen wurden im Tempel der Hera in Olympia für die Wettkämpfer aufbewahrt.“; vgl.H. A. HARRIS, GAA, $74 \mathrm{f}$.
} 
Bei fast all den angesprochenen Aspekten finden wir bei den Gladiatoren ein ebenso, wenn nicht noch größeres Bestreben um Chancengleichheit, ${ }^{51}$ zumal die Gefahr für Leib und Leben hier am größten war. ${ }^{52}$ Die Zusammenstellung der Kampfpaare (compositio ${ }^{53}$ ) erfolgte nicht durch Los, sondern wurde vom Spielgeber und dem lanista vor Beginn der Veranstaltung festgelegt und öffentlich bekannt gemacht. ${ }^{54}$ Bei der compositio kannte man die Möglichkeit des Gefechts von Gladiatoren desselben Kampftypus oder zweier Kämpfer unterschiedlicher armatura. Im ersteren Falle war die Chancengleichheit von vornherein gewahrt; bei unterschiedlicher armatura hatte man solche Kampftypen gegeneinander gestellt, bei denen sich Vor- und Nachtei ${ }^{55}$ gegenseitig neutralisierten, ${ }^{56}$ was spannungsreiche und chancengleiche Kämpfe bot. ${ }^{57}$ Ferner kämpften überwiegend Gladiatoren gegeneinander, die in etwa über die gleiche Wettkampferfahrung verfügten, was sich in der Zahlenangabe ihrer Kämpfe bzw. Siege widerspiegelte. ${ }^{58}$ Unmittelbar vor Kampfbeginn kam es zu einer Prüfung der Waffen (probatio armarum) durch das Kampfgericht. ${ }^{59}$ Auch waren häufig zur Einhaltung der Kampfregeln (leges pugnandi) gleichzeitig zwei Kampfrichter (summa und secunda rudis) im Einsatz. ${ }^{60}$ Dies mag auch z. T. in der Schnelligkeit des Kampfgeschehens

\footnotetext{
${ }^{51}$ Vgl. D. RAMBA, Gladiatur, 55.

${ }^{52} \mathrm{Vgl}$. G. STAAB, in: ZPE 161, 2006, 40: „..., unter den nach strengem Ehrenkodex abgelaufenen Kämpfen zwischen Gladiatoren, die oft als Kameraden in derselben Gemeinschaft miteinander trainierten und lebten. “

${ }^{53} \mathrm{Vgl}$. M. JUNKELMANN, Spiel, 130: "Sie (die compositio, der Verf.) wurde vom editor vorgenommen, der sich dabei, je nach persönlicher Sachkenntnis, vom lanista und den doctores (Trainern) beraten ließ.“.

${ }^{54}$ Zum libellus muneris vgl. M. JUNKELMANN, Spiel, 130.

${ }^{55}$ Geringerer Schutz, aber höhere Beweglichkeit stand gepanzerter Statik gegenüber.

${ }^{56}$ Vgl. L. ROBERT, Gladiature, 295; D. RAMBA, Gladiatur, 56.

${ }^{57}$ Vgl. M. JUNKELMANN, Spiel, 130.

${ }^{58} \mathrm{Vgl}$. L. ROBERT, Gladiature, 295.

${ }^{59}$ Vgl. M. J UNKELMANN, Spiel, 130 u. 133.

${ }^{60}$ Vgl. M. JUNKELMANN, Spiel, $134 \mathrm{f}$.
} 
bzw. dessen eventuell schwerwiegenden Folgen (lebensgefährliche Verletzungen oder sogar Tod) begründet liegen.

Bei den hippischen Agonen der Griechen ist es vor allem die ausgeklügelt gestaltete Startvorrichtung, ${ }^{61}$ die für ein hohes Maß an Chancengleichheit sorgte. $^{62}$ Bei den Wagenrennen der circensischen Spiele war es in erster Linie die Aufgabe der Startboxen (carceres), Fehlstarts unmöglich zu machen. ${ }^{63}$

Von der Athletik und den hippischen Agonen über die Gladiatorenkämpfe bis zu den circensischen Wagenrennen wurde im gesamten Sportbetrieb der griechisch-römischen Antike dem Prinzip der Chancengleichheit größter Wert beigemessen.

\footnotetext{
${ }^{61}$ Vgl. H. A. HARRIS, SGR, $161 \mathrm{ff}$.

${ }^{62} \mathrm{Vgl}$. I. WEILER, Sport, $203 \mathrm{f}$.: ,....ausführlich beschriebene komplizierte Startanlage

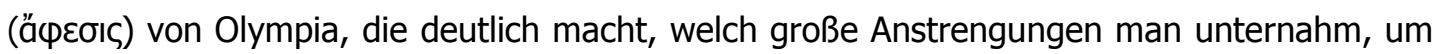
die Chancengleichheit der Teilnehmer an den hippischen Agonen gewährleisten zu können.“ ${ }^{63}$ Vgl. J. H. HUMPHREY, Circuses, $132 \mathrm{ff}$.
} 


\section{$3.4 \quad$ Leistung}

Leistung und deren Erfassung war in der griechisch-römischen Antike in allen Bereichen des Lebens stets präsent und auf vielfältige Weise möglich. ${ }^{1} \mathrm{Im}$ Bereich des Sports gab es zwei verschiedene Formen der Leistungsmessung.

Für die Leistungsmessung nach Weite, Gewicht und Geschwindigkeit, dem c(entimeter)-g(ram)-s(econd)-System, kamen im Sport der griechischrömischen Antike die Leichtathletik, Gewichtheben und das wettkampfmäßig betriebene Bogenschießen in Frage. ${ }^{2}$

Im Bereich der Athletik sind es die drei technischen Disziplinen Weitsprung, Speer- und Diskuswurf, die uns genaue Zahlenangaben zu erzielten Weiten liefern, indem man die erzielten Weiten mittels Markierung mit einem Pflock festhielt. ${ }^{3}$ Auf zahlreichen Vasenbildern sehen wir, wie Akteure (Sportler, Trainer, Funktionär) die erzielte Weite markieren ${ }^{4}$. Anschließend konnten die erzielten Weiten von der Absprung- bzw. Abwurfstelle bis zur Markierung genau vermessen werden. ${ }^{5}$ Wir kennen Sportanlagen, bei denen an einer Seite des Stadions Weitenmarkierungen angebracht waren, die das Vermessen der Weite erleichterten. ${ }^{6}$ Besonders viel diskutiertes Interesse

\footnotetext{
${ }^{1}$ F. BERNSTEI N, Ludi publici, Stuttgart 1998, 268: „Besser, eher noch „der Beste“ zu sein, war als immanentes Wesensmerkmal des Leistungsethos das natürliche Ziel des nobiles. Doch in diesem ,agonistischen` Momentz lag eine besondere Sprengkraft für die weitere Entwicklung des öffentlichen Spielwesens.“
}

${ }^{2}$ Vgl. H. ALTROCK, Die geistigen Bindungen des Sports in der abendländischen Kultur, in: Stud Gen 13, 1960, 25: „Im Sport wird die körperliche Leistung gemessen, absolut imC-G-SSystem oder relativ nach einer Punktwertung."

H. EICHBERG, Leistung, 17 ff; A. GUTTMANN, Ritual, 48.

${ }^{3}$ Vgl. R. PATRUCCO, Sport, 134: „Non solo infatti l'uso di ońnata dimostra la nozione acquisita di misurazione del lancio, di una valutazione relative e assoluta, in definitiva del record sportivo."

${ }^{4}$ Vgl. J. JÜTHNER, $A L$ 2, 220 f.; R. PATRUCCO, Sport, Fig. 74; V. OLIVOVÁ, Sport, 115.

${ }^{5}$ Vgl. R. PATRUCCO, Sport, 77.

${ }^{V}$ gl. E. K. BORTHWICK, in: JHS 84, 1964, 50. 
erlangte eine inschriftliche Weitenangabe von 55 Fuß $(\sim 15 \mathrm{~m})$ beim Weitsprung, die jedoch realistisch erscheint, wenn man darunter die Gesamtweite eines in Folge durchgeführten fünffachen Standsprunges versteht. ${ }^{7}$ Da aber die drei technischen Disziplinen gewöhnlich nur im Fünfkampf (Pentathlon) praktiziert wurden, wobei nicht in erster Linie die Weite, sondern die Platzierung für ein Weiterkommen bzw. den Gesamtsieg entscheidend war, wurden nur außerordentliche Leistungen gemessen und als rekordverdächtig der Nachwelt überliefert. ${ }^{8}$ Dass man generell an hohen Weiten interessiert war, zeigt die Verwendung von Sprunggewichten

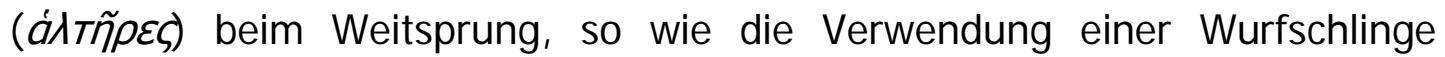
(áyúd $\eta$ ) beim Speerwurf und einer stimulierenden Musikbegleitung. ${ }^{9}$

Das Gewichtheben war bei Griechen und Römern kein eigenständiger Wettkampfsport. Man kannte aber Veranstaltungen, bei denen man durch Heben oder Bewegen großer Gewichte nicht nur den Nachweis physischer Kraft demonstrieren konnte, sondern bei denen auch der Stärkste ermittelt werden sollte. ${ }^{10} \mathrm{Im}$ Training für unterschiedliche Sportarten wurden zur Verbesserung der Physis mit z. T. genormten Gewichten geübt. ${ }^{11}$

Beim Bogenschießen kannte man Wettbewerbe im Zielschießen auf Figuren und Scheiben (z. T. vom galoppierenden Pferd aus), wobei die Präzision des Schusses zur Siegerermittlung entscheidend war. Beim wettkampfmäßig betriebenen Weitschießen, bei dem man spezielle Bögen und Pfeile benutzte, wurden die erzielten Distanzen genau gemessen, in Ranglisten erfasst und somit der Sieger ermittelt. ${ }^{12}$ Die Messung der Geschwindigkeit kam beim

\footnotetext{
${ }^{7}$ Vgl. J. EBERT, Pentathlon, $61 \mathrm{ff}$.

${ }^{8} \mathrm{Vgl}$. I. WEI LER, Sport, $157 \mathrm{ff}$.

'Vgl. W. DECKER, Sport, 152.

${ }^{10}$ Vgl. N. B. CROWTHER, in: G\&R 24, 1977, 111 ff. (=Athletika 269 ff.); W. E. SWEET, Sport, 104 ff.; W. DECKER, Sport, 122.

${ }^{11}$ Vgl. N. B. CROWTHER, in: G \& R 24, 1977, 115 ff. (=Athletika 274 ff.).

${ }^{12} \mathrm{Vgl}$. L. MORETTI, IAG, 82 ff.; I. WEI LER, Sport, 198.
} 
Laufsport und theoretisch beim Bogenschießen vor.Beim Laufsport haben wir trotz der Vielfalt an Laufkonkurrenzen nur bedingt Zeitangaben über erbrachte Leistungen. Zur Ermittlung der Leistung bei Kurz- und Mittelstrecken verfügte man nicht über entsprechende Zeitmessgeräte. ${ }^{13}$ Was die Siegerermittlung bei den meisten Konkurrenzen anbelangt, so erfolgte sie durch Augenschein des Kampfgerichts. Die Überlegenheit des Siegers drückte sich im sichtbaren Abstand zu seinen Konkurrenten aus; bei einem äußerst knappen Entscheid konnte es zu Wiederholungsläufen kommen. Anders verhält es sich bei Angaben zu Laufleistungen über längere Distanzen. Wir haben einige Leistungsangaben, bei denen wir sowohl die Distanz als auch die dafür benötigte Zeit kennen. So ist ein Läufer nach seinem Sieg in Olympia nach dem Wettkampf noch zu seinem $100 \mathrm{~km}$ entfernten Heimatort gerannt. In einem anderen Fall war es ein Zuschauer, der bei den Eröffnungsfeierlichkeiten in Olympia dabei sein durfte, berichtete am selben Abend davon seinen Landsleuten im 140 km entfernten Epidauros. In Rom war ein Erwachsener von morgens bis abends durch den Circus Maximus gelaufen und hatte eine Strecke von "CLX passuum" (= $237 \mathrm{~km})$ zurückgelegt. $^{14}$

Die Messung der Geschwindigkeit kam beim Laufsport und beim Bogenschießen in Betracht.

Beim Bogenschießen stellte man zur Ermittlung der Fluggeschwindigkeit eines Pfeils geradezu wissenschaftliche Überlegungen zu einem Experiment an: Eine bestimmte Anzahl von schussbereiten Bogenschützen wurde in bestimmten Abständen hintereinander postiert. Sobald der Pfeil des hinten stehenden Schützen den Vordermann passierte, gab dieser seinen Pfeil frei u. s. w. Bei Berechnung der Relation von Zeit und Entfernung wollte man so die Geschwindigkeit ermitteln. ${ }^{15}$

\footnotetext{
${ }^{13} \mathrm{Vgl.} \mathrm{J} \mathrm{.} \mathrm{JÜTHNER,} A L$ 1, 129.

${ }^{14} \mathrm{Vgl}$. L. FRIEDLÄNDER, SG 2, 24; Y. KEMPEN, Krieger, $91 \mathrm{ff}$.

${ }^{15} \mathrm{Vgl}$. O. v. FLESCHENBERG, in: WS 60, 1942, 52.
} 
Die Darstellung persönlicher Qualitäten wie auch die überzeugende Art und Weise von Erfolgen oder Siegesserien führten die Spitzensportler in vielfältiger Weise an. ${ }^{16}$ So brüsteten sich manche Sportler mit ihren physischen oder psychischen Qualitäten ${ }^{17}$ oder hielten sich in beiden Belangen der Konkurrenz für überlegen. Auch ihre besonders gute Technik ${ }^{18}$ und ihr überaus kluges taktisches Verhalten hoben sie hervor. Im konkreten Fall begann die Auflistung der Leistungen mit der Erwähnung der Teilnahme bzw. bei bedeutenden Festen der erfolgreichen Qualifikation für das Hauptfeld. Man führte weiter an, dass man eine oder mehrere Runden ${ }^{19}$ überstanden bzw. Gegner ${ }^{20}$ bezwungen hatte. Das Erreichen des Finales konnte den Sportler schon mit Stolz erfüllen, besonders wenn er im Verlauf der Veranstaltung nicht durch ein Freilos begünstigt worden war. Eine sehr knappe, vielleicht auch sehr unglückliche oder sogar durch eine Kampfrichterfehlentscheidung erlittene Finalniederlage musste nicht als Schmach empfunden werden. Zwar galt das ganze Streben dem Sieg, doch war ein zweiter Platz nicht nur ein gewisser Beweis eigener Leistungsstärke, sondern wurde bei einigen Veranstaltungen mit einer entsprechenden Belohnung bedacht. Bei verschiedenen Veranstaltungen konnten neben den Siegern und Zweitplatzierten auch die auf die folgenden Plätze verwiesenen Sportler noch ansehnliche Preise einstreichen. Ein Finale mit unentschiedenem Ausgang konnte nicht nur Anerkennung finden, sondern auch mit Gewinn verbunden sein. Hatte man in den Anfängen bei einem unentschiedenen Finalkampf den ausgesetzten Siegpreis der Gottheit

\footnotetext{
${ }^{16}$ Vgl. H.-W. PLEKET, GL 2, 302: „Worauf es vor allem ankommt, ist die Tatsache, daß schon in Pindars Oden (s. Ol. 13, 37-38; 11,92) die Tendenz belegt wird, daß man mit dem Sieg allein noch nicht zufrieden war, sondern daß man inn mit anderen Werten kombinierte.“

${ }^{17}$ Vgl. B. SCHRÖDER, Sport, 147; W. RUDOLPH, Kampfsport, $27 \mathrm{f}$.

${ }^{18}$ Vgl. H.-W. PLEKET, GL 2, 304: „Das Wort techne ... bezieht sich hier auf einen Boxer oder einen Ringer, der sich mehr auf technisches Geschick als auf brutale Stärke verläßst.“

${ }^{19}$ Vgl. N. B. CROWTHER, in: Stadion 18.1, 1992, 68 ff. (=Athletika 215 ff.).

${ }^{20}$ Vgl. N. B. CROWTHER, in: Nikephoros 6, 1993, 39 ff. (=Athletika 171 ff.);
} 
geweiht, so wurde später eine Aufteilung unter den Finalisten vorgenommen. $^{21}$

Den Berufssportlern konnte nicht daran gelegen sein, sich bis zur völligen Erschöpfung zu verausgaben und besonders in der Schwerathletik erhebliche Blessuren oder Verletzungen zu riskieren, was eine Unterbrechung oder das Ende ihrer Wettkampftournee hätte bedeuten können. Dadurch kam es (ohne Bestechung) zunehmend häufiger zu unentschieden endenden Finalkämpfen.

Allein der Sieg im Finale einer bedeutenden Veranstaltung brachte schon für sich Ruhm und Ehre. Dennoch war man bemüht, ihm zusätlich besonderen Glanz zu verleihen. So führte man an, dass man alle Runden hatte bestreiten müssen und erwähnte namentlich den bezwungenen Finalgegner, wenn dieser zu den Spitzenathleten zählte. Besondere Anerkennung beanspruchten Sportler, die - sei es aus freien Stücken oder auf Beschluss des Kampfgerichts - in einer höheren Altersklasse antraten oder dazu bestimmt waren, und dennoch sich gegen die gesamte Konkurrenz hatten durchsetzen können. ${ }^{22}$

Innerhalb der einzelnen Sportarten und -disziplinen kannte man eine Vielzahl von Begriffen und Formulierungen, durch die man seine Klasse und Dominanz zum Ausdruck bringen konnte. ${ }^{23}$ So führten erfolgreiche Ringkämpfer an, dass sie nicht auf die Knie oder Schulter gezwungen worden waren, dass man sie nicht um die Hüfte habe fassen können oder nicht in eine kritische Situation gebracht hatte. ${ }^{24}$ Überhaupt hatten sie ganz souverän

\footnotetext{
${ }^{21}$ Vgl. L. MORETTI, IAG, 53; N. B. CROWTHER, in: Nikephoros 13, 2000, 130 ff. (=Athletika 302 ff.).

${ }^{22}$ Vgl. N. B. CROWTHER, in: Phoenix 42.4, 1988, 304 ff. (=Athletika 87 ff.).

${ }^{23}$ Vgl. H.-W. PLEKET, in: Stadion 1, 1975, 79: "There is a tendency to add, so to speak, a "Surplus-value" to that of the victory in itself."

${ }^{24} \mathrm{~J}$. JÜTHNER, $A L$ 1, 130, sieht in Erfolgen, die auf bemerkenswerte Weise (ákovití und

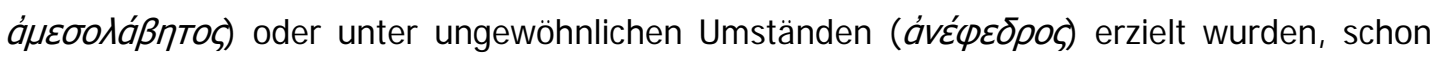
Rekorde.
} 
ihre Kämpfe in nur drei von fünf möglichen Runden gewinnen können. Boxer triumphierten mit ihren Defensivkünsten, die ihre Gegner schier zur Verzweiflung brachten oder verglichen ihr Aussehen mit dem von Läufern, da sie keine schweren Schläge hatten einstecken müssen. Pankratiasten prahlten mit einer Anzahl von Aussagen in einer weder-noch-Formel ( $\mu \eta^{\prime} \varepsilon$... $\mu \eta^{\prime} \tau$ ) über ihre Dominanz. ${ }^{25}$

Ein kampfloser Sieg wurde mit dem Zusatz ákoviti ${ }^{26}$, d. h. ohne Staub, angezeigt. Diese Aufgabe konnte sich auf einen Kampf, eine Veranstaltung oder eine Siegesserie beziehen. Ein Kuriosum stellen die ákovití-Siege eines uns unbekannten Schwerathleten dar: Er muss von solch stattlicher, ja geradezu Furcht erregender Statur gewesen sein, dass, nachdem er sich entkleidet hatte, die gesamte Konkurrenz vom Wettkampf Abstand nahm. Somit hatte er ohne Kampf (ákoviti) gewonnen. ${ }^{27}$ Ebenso konnte mit den

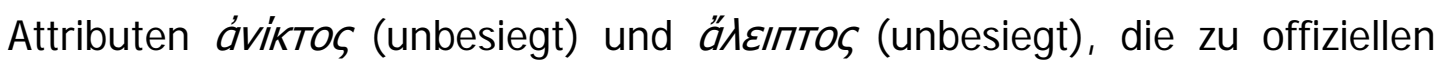
Epitheta (Titel) geworden waren, manifestiert werden, dass man keine Niederlage erlitten hatte. Ein weiterer Aspekt einer erfolgreichen Sportlerlaufbahn war die Angabe von Siegesserien, was mit dem Epitheton ката́ то $\dot{X} \tilde{\eta} \varsigma$ (nacheinander) ${ }^{28}$ angezeigt wurde. Solche Siegesserien konnten sich auf ein und dasselbe Fest beziehen, das man über Jahre oder Jahrzehnte erfolgreich besucht hatte, was somit auch eine Serie bezüglich der Altersklassen mit sich bringen konnte. Die Serienangabe konnte aber auch Bezug haben auf siegreich bestrittene Wettkämpfe bei Spielen, die man im Rahmen der Wettkampftournee unmittelbar nacheinander aufgesucht hatte.

\footnotetext{
${ }^{25}$ Vgl. J.-Y. STRASSER, zu M. Aur. Dém. Damas, in: BCH 127, 2003, 251 ff.; W. DECKER, Sport, 116.

${ }^{26}$ Vgl. H.-W. PLEKET, GL 2, 302: „... Athleten, die akoniti gewannen, d. h. ohne gekämpft zu haben, weil alle Gegner vor Beginn der Spiele aus Angst vor dem ,Superstar' einen Rückzug machten.“
}

${ }^{27}$ Vgl. J. JÜTHNER, $A L$ 1, 131.

${ }^{28}$ Vgl. M. LÄMMER, Olympien, 60. 
Die Aufwertung der Siege durch zahl- und variantenreiche Epitheta ornantia $^{29}$ war bis zum Ausgang der Spätantike auffällig häufig präsent. ${ }^{30}$

${ }^{29}$ Vgl. L. ROBERT, in: RPh 1967, 17; M. POLIAKOFF, Kampfsport, 147.

${ }^{30}$ Vgl. H.-W. PLEKET, GL 2, 303: ,... mögen in römischer Kaiserzeit ... die Worte neu und das Vokabular reicher geworden sein. Aber die Haltung, die in den Worten zum Ausdruck kommt, ist die gleiche wie die in Pindars Oden." 
Zahlen waren für die Welt der griechisch-römischen Antike von allergrößter Bedeutung. ${ }^{1}$ Nahezu alle Wissenschaften standen in einem mehr oder weniger starken Abhängigkeitsverhältnis bzw. basierten auf dem Numerus. ${ }^{2}$ Auch die meisten gesellschaftspolitischen Lebensbereiche waren von der Zahlenwelt durchdrungen oder beherrscht: Politiker führten gern in Inschriften die Zahl ihrer politischen und kultischen Ämter an, Militärs machten nach Kriegen Angaben über die Menge der Kriegsbeute und der Zahl der Gefangenen, und das prosperierende Wirtschaftsleben fand seinen Niederschlag in entsprechenden Tabellen und Statistiken. Religionen waren geprägt von mystischen Zahlen. Kunst und Musik waren von numerischen Werten durchzogen. Spielgeber ${ }^{3}$ nannten in ihren Tatenberichten die Zahl der Feste bzw. Festspieltage, der Teilnehmer oder dargebotenen (exotischen) Tiere. Die Jahreszählung erfolgte nach Olympiaden, ${ }^{4}$ zu Beginn unserer christlich-abendländischen Zeitrechnung fand eine Volkszählung ${ }^{5}$ statt, und im Privatleben kannte man das Durchzählverfahren bei den männlichen Nachkommen. ${ }^{6}$

\footnotetext{
${ }^{1}$ W. TSCHI RK, Vom Spiegel des Universums. Eine Geistesgeschichte der Mathematik, Wien 2007, 11: „Für uns begann die Mathematik mit den Griechen.“; S. CUOMO, Ancient Mathematics, London/New York 2001, bes. 143 ff.; H. GERICKE, Mathematik im Abendland. Von den römischen Feldmessern bis zu Descartes, Wiesbaden 1990, bes. 37 f.

${ }^{2}$ Vgl. E. REICHMANN, Herrschaft der Zahl. Quantitatives Denken in der deutschen Aufklärung, Stuttgart 1968, 9; J. McCLELLAND, The Number of Reason: Luck, Logic, and Art in Renaissance Conceptions of Sport, in: J. M. CARTER/A. KRÜGER (Hrsg.), Ritual, 53 ff.

${ }^{3}$ Augustus, Res gestae, 23.

${ }^{4}$ W. DECKER, Sport, 58: „Wenn in der antiken Geschichtsschreibung Olympiaden (d. h. der Zeitraum von vier Jahren zwischen der Abhaltung zweier Olympien) zu Datierungszwecken aufgeführt werden, ist der Erste im Stadionlauf der eponyme Sieger.“

${ }^{5}$ Evangelium Lukas, 2, $1 \mathrm{f}$.

${ }^{6}$ R. HARDER, Eigenart, 5: „In Rom kann man die Kinder einfach numerieren: Quintus, der Fünfte, Decimus, der Zehnte, Postumus, der Nachgeborene.“
} 
Diese fast überall praktizierte Form der zahlenmäßigen Erfassung und Darstellung von Gegebenheiten und Leistungen fand einen besonders intensiven Niederschlag im Sport. ${ }^{7}$ Wo immer es möglich erschien, wurde die Übertragung von der Qualität der sportlichen Leistung in die Quantität der Zahl vollzogen. ${ }^{8}$ Dieses ermöglichte nach konkreter bzw. präziser Leistungserfassung - trotz gewisser zeitlicher und lokaler Unterschiede - eine objektive Vergleichbarkeit. ${ }^{9}$

In der Athletik waren die Zahlenangaben nach Erfolgen in Periodosspielen, Heiligen Spielen, die mit einem Siegpreis ausgestattet waren, reinen Geldoder Sachpreisspielen, Spielen, die in bestimmtem zeitlichen Abstand ausgetragen wurden, außerordentlichen Veranstaltungen und zuweilen auch weniger bedeutenden, meist lokalen Festen, gegliedert. ${ }^{10}$ Die Angaben konnten in knapper und prägnanter Form oder in ausführlichen, teils mit Epitheta ornantia aufgewerteten, Auflistungen erfolgen. Die Angabe bzw. Aufteilung der Erfolge konnte auch ganz speziellen Aspekten unterworfen sein. Man führte gesondert die Zahl seiner Heimsiege an oder nannte die Erdteile, in denen man siegreich gewesen war. Die Summe der Siege konnte in Bezug bzw. Verbindung zu Trainer, Geschwistern und dem Elternhaus wie auch der Heimatgemeinde oder der ethnischen Gruppe, der man angehörte, dargeboten werden. ${ }^{11}$

Die Verfasser von Siegesinschriften forderten Wanderer auf, vor dieser Inschrift zu verharren und die Siege zu zählen, auch wenn es mühsam ist. ${ }^{12}$ Eine weitere Form des Ausdrucks für die Wertschätzung besonders hoher

\footnotetext{
${ }^{7}$ Vgl. A. GUTTMANN, Ritual, 57.

${ }^{8}$ Vgl. H. EICHBERG, Der Weg des Sports in die industrielle Zivilisation, Baden-Baden 1973, 11; A. GUTTMANN, Ritual, 171.

${ }^{9}$ Zu Parallelen von modernen und antiken Siegverzeichnissen K. KRAMER, Studien, 3: „Die Ähnlichkeit ist nicht beabsichtigt, sondern im Wesen der Sache begründet.“

${ }^{10}$ Vgl. H. W. PLEKET, in: Stadion 1, 1975, 49 ff.

${ }^{11} \mathrm{Vgl}$. H. BUHMANN, Sieg, $53 \mathrm{ff}$.

${ }^{12} \mathrm{Vgl}$. J. EBERT, Epigramme, Nr. 79 (M. Aurelius Heras aus Chios).
} 
Erfolgszahlen liegt in Äußerungen, die nicht konkret fassbar sind, aber auf eine erwünschte hohe Summe an Siegen schließen lassen sollen. So finden sich vage Angaben wie etwa, dass man oft gesiegt habe, und man überhaupt so viele Triumphe hatte feiern können, für die der Platz nicht ausreiche, alle anzuführen. Ferner spricht man von unzähligen Erfolgen oder aber, dass die Siege so zahlreich seien wie die Kiesel am Strand. ${ }^{13}$

Man kannte auch bezüglich der Leistungen und Erfolge gemischte Auflistungen, wie sie uns beispielhaft die Gladiatoreninschriften liefern. Neben Namen und Kampfgattung des Gladiators werden wir über Zahl der Kämpfe, Siege, Unentschieden und Niederlagen - die ohne (tödliche) Konsequenzen für den Unterlegenen geblieben waren - detailliert informiert. ${ }^{14}$ Auf Gladiatorenreliefs findet sich gelegentlich um den meist mit Palmenzweig dargestellten Gladiator eine Anzahl von Siegeskränzen. Bei gleichzeitiger Inschrift mit Zahlenangabe ist diese meist deckungsgleich mit der Zahl der dargestellten Siegessymbole. ${ }^{15}$

Bei der Mehrzahl der Ballspiele waren Spielidee und Siegermittlung ohne Zählen oder konkrete Zahlenwerte nicht durchführbar. Man nutzte sogar zum Zählen des aktuellen Spielstandes und zur Feststellung des Siegers speziell dafür abgestelltes Personal. ${ }^{16}$

Beim zahlenmäßigen Nachweis der Leistungen und/oder Erfolgen bei den hippischen Agonen finden wir eine Mehrzahl von Parallelen zu Angaben aus dem Bereich der Athletik. Siegerkataloge gaben Festort, Status der

\footnotetext{
${ }^{13}$ J. EBERT, Epigramme, 18: „J etzt findet sich auch schon die ,Abbruchformel', offenbar dem Epinikion, d.h. der chorlyrischischen Siegerdichtung, entlehnt, durch die sich der Epigrammdichter die Aufzählung aller, zumal der unbedeutenderen Agone ersparte, den Ausfall aber meist durch hyperbolische Fassung der Formel ausglich.“

${ }^{14}$ Vgl. P. SABBATINI TUMOLESI, Gladiatorum, 96.

${ }^{15}$ Vgl. P. SABBATINI TUMOLESI, Gladiatorum, 104.

${ }^{16}$ Vgl. S. MENDNER, Ballspiel, $85 \mathrm{ff}$.
} 
Veranstaltung und Disziplin an, bevor die Zahl der Siege folgte. Die Zahlenangaben konnten sich auf Einzelpersonen, wie auch auf Familien oder ethnische Gruppen beziehen. ${ }^{17}$

Geradezu eine Manie ${ }^{18}$ für tabellarische Leistungsnachweise finden wir in den Inschriften römischer Wagenlenker. ${ }^{19}$ Zuerst führten sie die Zahl ihrer Starts - chronologisch aufgelistet boten sie einen guten Einblick in den Verlauf ihrer Karriere - oder schlicht in einer Gesamtzahl an. Dieser Angabe konnte die Zahl der Siege, zweiter, dritter und vierter Platzierungen folgen. Sogar Wiederholungsläufe und Rennen, die man erfolglos, d. h. ohne Preisgeld absolviert hatte, wurden aufgezählt. Die meisten Zahlenangaben konnten der Unterteilung in die der vier Rennparteien (factio albata, prasina, russea und veneta) unterliegen, für die fast alle namhaften agitatores gefahren waren. Ein weiteres Kriterium der Unterteilung der Leistungen war die Gespannform, wobei Erfolge mit dem Zweigespann (biga) dem Nachwuchs vorbehalten, die Rennen mit dem Viergespann (quadriga) üblich waren, und Rennen von Gespannformen mit mehr als vier Pferden (septemiuge, octoinge und decemiuge) eine Ausnahme darstellten. Eine weitere Zahlenangabe teilte die Siege bezüglich der am Rennen teilnehmenden Wagen pro factio ein. Meist war für jede der vier Rennparteien ein Gespann am Start (certamen singularum), Läufe mit vier Wagen pro Partei (certamen quaternarum) stellten eine absolute Ausnahme dar. Auch die Art und Weise, in der man gesiegt hatte, wurde differenziert aufgeführt: Von einem Start-Ziel-Sieg bis hin zu einem äußerst knappen Triumph im Finish wurden alle Rennverläufe bzw. -ausgänge exakt aufgelistet. Ein weiterer Aspekt der Zahlenangaben konnte sich auf die Höhe der Gewinnsummen beziehen. Hierbei erwähnte man nicht die gewöhnlichen Siegespreise, sondern konzentrierte sich auf die außerordentlichen Prämien - wahrscheinlich nur für besondere Rennen mit

\footnotetext{
${ }^{17}$ Vgl. J. EBERT, Epigramme, 62.

${ }^{18} \mathrm{Vgl}$. D. RAMBA, Recordmania, $34 \mathrm{f}$.

19 H. A. HARRIS, SGR, 194: "From the beginning of the Christian era statistics of performances in the Circus were as carefully kept as they are on the modern Turf."
} 
einem exklusiven Aufgebot an Fahrern. Die praemia maiora bewegte sich zwischen 30.000 und 60.000 Sesterzen und machte erfolgreiche Lenker zu Berühmtheiten der antiken Sportszene und zu sehr wohlhabenden Männern. Da die Inschrift zeitnah die sportlichen Leistungen dokumentierte, gibt es über die Korrektheit der konntenn keine Zweifel. ${ }^{20}$ Nicht nur die Veranstalter oder Leiter der Wettkämpfe erfassten die Sieger, auch von mehreren Organisationen, Personen und Gemeinschaften wurden auf unterschiedlichste Weise die Leistungen und/oder Siege der Sportler festgehalten. So führten Athletenverbände Buch über die Erfolge ihrer Mitglieder, was sich deutlich in den Diplomen ${ }^{21}$ der Vorstandsmitglieder ablesen lässt. Rennparteien ${ }^{22}$ führten akribisch Listen über die Leistungen von Wagenlenkern und allen am Rennen beteiligten Pferde. ${ }^{23}$ So hielten auch der Sportler selbst und sein Trainer die Leistungen fest, wie auch die Familien und Heimatgemeinden ${ }^{24}$ mit Stolz die Leistungen ihrer Söhne aufzeichneten. Auch Prosaisten (z. B. Dio Cassius), Satiriker (z. B. Lucillius) und Exegeten (z. B. Pausanias) lieferten uns dienliches Zahlenmaterial. Schließlich waren es - wenn auch auf ganz unterschiedliche Art und Weise - die Panegyriker (z. B. Pindar ${ }^{25}$ ) und die Verfasser von Graffiti ${ }^{26}$, deren Werke weitere numerische Quellen darstellen.

\footnotetext{
${ }^{20}$ Vgl. L. ROBERT, in: AE 1966, 112 ff.; DERS., Lucillus, 183 n. 2; H. W. PLEKET, GL 2, 302.

${ }^{21} \mathrm{Vgl}$. H. W. PLEKET, Some Aspects of the History of the Athletic Guilds, in: ZPE 10, 1973, $197 \mathrm{ff}$.

${ }^{22} \mathrm{CIL}$ 6, 10048=ILS 5287: Z 13 (Actis continetur ...); vgl. A. CAMERON, Porphyrius, 52.

${ }^{23}$ Vgl. G. BIANCO, in: RIL 111, 1972, $317 \mathrm{ff}$.

${ }^{24} \mathrm{Vgl}$. H. BUHMANN, Sieg, $53 \mathrm{f}$.

${ }^{25}$ E. WELSKOPF, Krise, 1486: „Es ist erfreulich, wie er (der Dichter Pindar, d. Verf.) es selbst herauszubringen weiß, daß ihm die Aufzählung lästig ist, die er doch nicht vermeiden darf.“

${ }^{26}$ Vgl. P. SABBATI NI TUMOLESI, Gladiatorum, 117.
} 
Allgemein bot der Bereich speziell des Leistungs- und Wettkampfsports ein interessantes Spektrum an Zahlenangaben, Statistiken aller Art und sogar Zahlenspielereien ${ }^{27}$.

27 Der zweite Olympiasieg des Schwerathleten Theogenes veranlasste ihn, seinen Sohn Disolympios (zweifacher Olympiasieger) zu nennen, vgl. L. MORETTI, Olympionikai, Nr. 201 u. 215; J. EBERT, Siegerepigramme, 122, n. 1. 


\subsection{Titel}

Der Wettkampfsport implizierte für die Teilnehmer immer das Streben nach erfolgreichem Abschneiden, nach Endkampfteilnahme und Finalsieg, ${ }^{1}$ und bei bedeutenden Festen war ein ganz besonderes Augenmerk auf die begehrten Titel gerichtet ${ }^{2}$.

Aus der Vielzahl der Feste mit sportlichem Programm ${ }^{3}$ waren es die panhellenischen (gesamtgriechischen) Spiele $^{4}$ der vier Festspielorte Olympia ${ }^{5}$, Delphi ${ }^{6}$, am Isthmos $^{7}$ und Nemea $^{8}$, die allen anderen Veranstaltungen, was Ruhm und Wertigkeit betraf, voranstanden ${ }^{9}$, obwohl es lediglich einen Kranz zu gewinnen gab. ${ }^{10}$ Alle Spiele dieser vier Austragungsorte standen unter dem Schutz einer bestimmten Gottheit: Die

\footnotetext{
${ }^{1}$ Vgl. E. N. GARDI NER, AAW, 101.

${ }^{2}$ Vgl. M. I. FINLEY/H. W. PLEKET, Spiele, 77.

${ }^{3}$ Vgl. T. KLEE, Geschichte, 29 ff.; I. WEI LER, Sport, 137 ff.

${ }^{4}$ Vgl. H. W. PLEKET, in: Stadion 1, 1975, 49 ff.
}

${ }^{5}$ Vgl. L. DREES, Olympia. Götter, Künstler und Athleten, Schorndorf 1967; H.-V. HERRMANN, Olympia. Heiligtum und Wettkampfstätte, München 1972; I. WEI LER, Sport, $105 \mathrm{ff.}$ W. DECKER, Sport, 34ff. ff.

${ }^{6}$ I. WEI LER, Sport, 128 ff., M. MAASS, Das antike Delphi. Orakel, Schätze und Monumente, Stuttgart 1997; S. PERROT, Les premiers concours des Pythia, in: Nikephoros 22, 2009, $7 \mathrm{ff}$.; W. DECKER, Sport, $41 \mathrm{ff}$.

7 I. WEILER, Sport, $131 \mathrm{ff} .$, M. KAJAVA, When did the Isthmian Games Return to the Isthmus?, in: CPh 97, 2002, 168 ff.; P. D. BALABANËS, Games and Sanctuaries in ancient Greece: Olympia, Delphi, Isthmia, Nemea, Athens, Los Angeles 2004; W. DECKER, Sport, $44 \mathrm{ff}$.

${ }^{8}$ I. WEILER, Sport, 134 ff., P. D. BALABANES, Games, 304 ff.; S. D. LAMBERT, Parerga //: The Date of the Nemean Games, in: ZPE 139, 2002, 72 ff.; S. G. MI LLER, Nemea. The Site and Museum, Athen 2004; W. DECKER, Sport, $46 \mathrm{ff}$.

${ }^{9}$ I. WEILER, Sport, 103 f., W. DECKER, Sport, 34.

10 W. DECKER, Sport, 33: „In Olympia war er vom heiligen Olivenbaum des Herakles, in Delphi vom Lorbeer Apollons geschnitten; in Nemea und am Isthmos vergab man Kränze aus Eppich (Sellerie) bzw. der Fichte Poseidons.“ 
Sieger bei diesen Spielen konnten sich mit dem Titel eines Olympioniken ${ }^{11}$, Pythioniken ${ }^{12}$, Isthmioniken ${ }^{13}$ und Nemeoniken ${ }^{14}$ schmücken.

Herausragend unter den Siegern dieser vier bedeutendsten Spiele, die man zusammenfassend $\pi \varepsilon \rho i o \delta o \zeta^{15}$ nannte, waren jene Sportler, die bei allen (vier) Veranstaltungen in derselben Disziplin und Altersklasse ${ }^{16}$ wenigstens einmal mit dem Kranz ausgezeichnet worden waren ${ }^{17}$. Sie konnten sich als

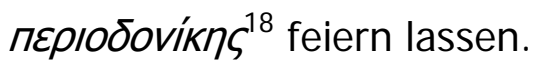

Der Periodonikentitel wurde erst im zweiten nachchristlichen Jahrhundert verliehen, obgleich man solch bemerkenswerte Leistungen schon seit dem 6 . J ahrhundert v. Chr. in der Veranstaltungschronik vermerkt hatte ${ }^{19}$.

Die Reihenfolge und der zeitliche Abstand der Erfolge bei den Periodosspielen war für die Erlangung des Periodoniken-Titels unbedeutend ${ }^{20}$, es war jedoch ${ }^{11}$ L. MORETTI, Olympionikai, I vincitori negli antichi agoni olimpici, Rom 1957; DERS.,
Supplemento al catalogo degli olympionikai, in: Klio 52, 1970, 295-303; DERS., Nuovo supplemento al catalogo degli olympionikai, in: Miscellanea Greca e Romana XII. Studi pubblicati dall'istituto italiano per la storia antica. Fasc.39, 1987, 67-91. W. DECKER, in: DNP 8, (1999), s. v. Olympioniken, $1188 \mathrm{f}$.

12 W. DECKER, in: DNP 10, (2001), s. v. Pythioniken, 667 f; E. MAROTI, Delphoi Pythia sportversenyeinek gyöztesei, Budapest 2000

${ }^{13}$ W. DECKER, in: DNP5, (1998), s. v. Isthmioniken, 1147 f; A. FARRINGTON, Isthmionikai: A Catalogue of Isthmian Victors (Nikephoros Beihefte 21), Hildesheim 2012.

${ }^{14}$ W. DECKER, in: DNP 8, (2000), s. v. Nemeoniken, 815 f; vgl. zu der Nemeonikenliste des GEORGI OS P. KOSTOUROS die Rezension von W. DECKER in: Nikephoros 22, 2009, 244-246 ${ }^{15}$ R. KNAB, Periodoniken, 4 f.; L. MORETTI, IAG, 34; E. MARÓTI, in: AAA 31, 1985, 335355; W. DECKER, Sport, 32: „Im modernen Tennissport kennt man Vergleichbares; hier ist demjenigen, der die vier wichtigsten Turniere in einer Saison (Wimbledon, Paris, New York, Melbourne) als Sieger beendet, der grand slam gelungen. “

${ }^{16}$ Bei den hippischen Agonen kannte man die Unterscheidung zwischen Pferden und Fohlen, das Alter des Reiters oder Lenkers war ohne Bedeutung.

${ }^{17}$ W. DECKER, Sport, 32.

18 J. JÜTHNER, $A L$ 1, 128; E. N. GARDENER, $A A W$, 106, sieht in einem Periodoniken einen „All-round champion“; H. SCHÖBEL, Olympia, 84 und F. BREIN, GL 2, 96, vergleichen Periodoniken mit heutigen Weltmeistern.

${ }^{19}$ Vgl. I. WEILER, Sport, 103. 
etwas Besonderes, wenn man den Titel in der kürzest möglichen Zeit

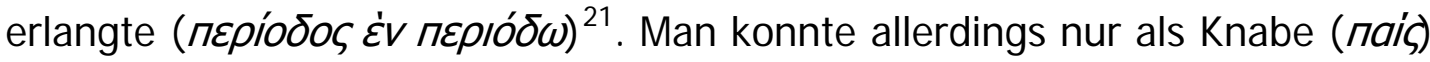
oder als Erwachsener (ávńp) Periodonike werden, da die Altersklasse der

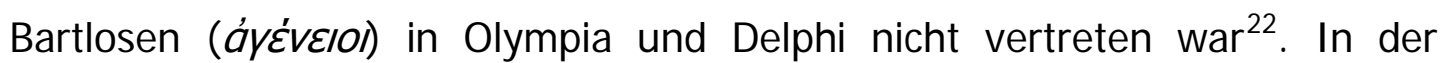
römischen Kaiserzeit wurde die Zahl von vier Periodosspielen durch Gründung neuer Festspiele auf sieben erweitert ${ }^{23}$. Kaiser Augustus gründete 27 v. Chr. die Aktischen Spiele zu Nikopolis ${ }^{24}$ und fügte $2 \mathrm{n}$. Chr. noch die Sebasta $^{25}$ in Neapel hinzu. Abgeschlossen wurde der Zyklus durch die Einführung der Kapitolien in Rom $^{26}$ im Jahre $86 \mathrm{n}$. Chr. durch Kaiser Domitian. Die Erweiterung des Zyklus schwächte das hohe Ansehen des Periodonikentitels ${ }^{27}$, da es wesentlich leichter für die Athleten war, vier Siege aus den nun angebotenen sieben Agonen zu gewinnen ${ }^{28}$. So kam besonderer Ruhm dem Ringer T. Aelius Aurelius Maro aus Seleukaia zu, der Mitte des

\footnotetext{
${ }^{20}$ R. KNAB, Periodoniken, 8.

${ }^{21}$ R. KNAB, Periodoniken, 9.

${ }^{22}$ R. KNAB, Periodoniken, 8.

23 J. u. L. ROBERT, in: REG 67, 1954, 114 f.; H. W. PLEKET, GL 2, 294; W. DECKER, Sport, $32 \mathrm{f}$.

${ }^{24}$ Suet. Aug. 18, 2; Dio Cass. 51, 1, 2; vgl.J. GAGÉ, Actiaca, in: MEFRA 53, 1936, 36 ff.; M. LÄMMER, Die Aktischen Spiele von Nikopolis, in: Stadion 12-13, 1986-1987, 27 ff.; O. PAVLOGIANNIS/E. ALBANIDIS/M. DIMITRIOU, The Aktia of Nikopolis: New Approaches, in: Nikephoros 22, 2009, $79 \mathrm{ff}$.
}

${ }^{25}$ Dio Cass. 54, 34, 1 f.;vgl. R. M. GEER, The Greek Games at Naples, in: TAPhA 66, 1935, 208 ff.; L. MORETTI, IAG, 175 f.; F. BREIN, GL 2, 136; H. A. HARRIS, SGR, 55; S. MÜLLER, Volk, 215; E. MARÓTI, Zur Regelung der Sportwettkämpfe der Sebasta in Neapel, in: Acta Antiqua 38, 1998, $211 \mathrm{ff}$.

${ }^{26}$ Vgl. I. LANA, I ludi capitoli Domiciano, in: RFIC 29, 1951, 145-160; B. RIEGER, Die Capitolia des Kaisers Domitian, in: Nikephoros 12, 1999, 171-203. H. A. HARRIS, SGR, 41, mit dem Hinweis auf Frauenwettbewerbe bei den Kapitolien.

${ }^{27}$ W. DECKER, Sport, 33.

${ }^{28} \mathrm{~J}$. EBERT, Epigramme, 234: „Im allgemeinen scheint man Siege an Festen der neuen Periodos dazu verwendet zu haben, die in der alten Periodos unvollständige Siegesserie zu ergänzen und dadurch den Titel пєрıобоviкnৎ zu erwerben." 
2. J ahrhunderts n. Chr. als erster die erweiterte Periodos gewinnen konnte ${ }^{29}$. Bei den hippischen Agonen wurde der Periodonikentitel den Besitzern der Pferde und nicht den Akteuren verliehen. ${ }^{30}$

Einen Titel, den Periodoniken mehrfach beanspruchen konnten, da alle Feste der periodos auch darunter fielen, war der des Hieroniken, d. h., eines

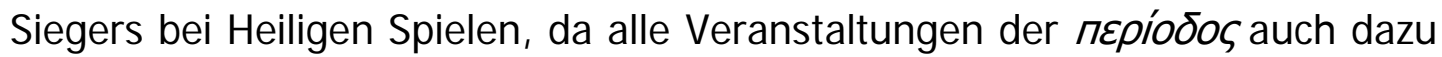
zählten. ${ }^{31}$ Auch hatten Sieger bei áy $\tilde{\omega} v \varepsilon \varsigma$ ispoí, da diese zu der nicht Titel

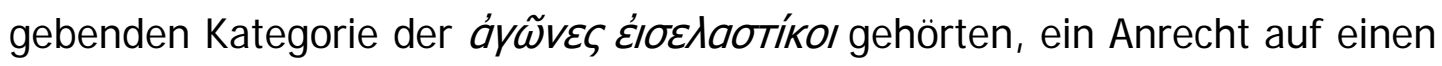
triumphalen Einzug in die Heimatgemeinde. ${ }^{32}$ Unter den zahlreich bezeugten Triumphzügen ragte der des Exainetos aus Agrigent heraus: ${ }^{33}$ Sein Einzug in die Heimatstadt war begleitet von 300 Persönlichkeiten, die inm auf 300 Schimmelzweigespannen ein Ehrengeleit boten. ${ }^{34}$

Ein später eingeführter, höherwertigerer Ehrentitel ist der des Pleistoniken ${ }^{35}$. Bei einer inflationären Vergabe von Heiligen Spielen und gleichzeitiger Abwertung des Titels Hieronikes ${ }^{36}$ schuf man mit dem Pleistonikentitel eine

\footnotetext{
${ }^{29}$ P. FRISCH, Der erste vollkommene Periodonike, in: EA 18, 1991, $71 \mathrm{ff}$.

${ }^{30}$ R. KNAB, Periodoniken, 8.

${ }^{31}$ Vgl. K. KRAMER, Studien, 133; H. W. PLEKET, in: MNIR 36, 1974, 57 ff.; I. WEI LER, Sport, 103.

${ }^{32}$ Vgl. J. u. L. ROBERT, in: REG 74, 1961, 149 f., Nr. 221; P. HERRMANN, Eine Kaiserurkunde der Zeit Marc Aurels aus Milet, in: MDAl (I) 25, 1975, $149 \mathrm{ff} .$, bes. 156 n. 23; H. S. VERSNEL, Triumphus, Leiden 1970, 155; R. MERKELBACH/J. STAUBER,
} Steinepigramme, Bd. 5, 331.

33 Diod. 13, 34; L. MORETTI, Olympionikai, Nr. 346.

${ }^{34}$ Vgl. E. N. GARDENER, AAW, 99.

${ }^{35}$ L. MORETTI, IAG, 163: ,... il titulo di пعрıоסоviкnৎ di cui si fregiava normalmente l'atleta chem, pur senza raggiungere la vittoria nella periodos, aveva nondimeno conseguito un rilevante numero di vittorie.“

${ }^{36}$ Vgl. R. MERKELBACH, in: ZPE 14, 1974, $95 \mathrm{f}$. 
neue qualitativ höherwertige Kategorie ${ }^{37}$, die auch entsprechend mehr an Vergünstigungen mit sich brachte ${ }^{38}$.

Ein Titel von hohem Ansehen ${ }^{39}$, verbunden mit bestimmten Vorrechten ${ }^{40}$,

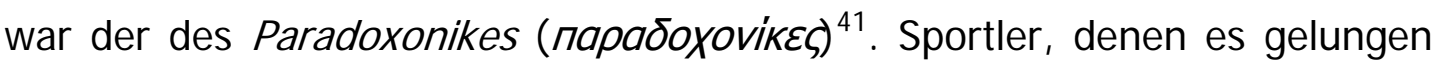
war, an einem Tag bei einem Fest einen Doppelerfolg zu erzielen, sei es in zwei Disziplinen, sei es in zwei Altersklassen, durften diesen Titel führen ${ }^{42}$.

Eine Sonderform des Paradoxonikes war der des Heraklessiegers ${ }^{43}$. Hier hatten es Schwerathleten geschafft, ihrem heroischen Vorbild nacheifernd, in Olympia an einem Tag Ringkampf und Pankration zu gewinnen ${ }^{44}$. Kapros von Elis $^{45}$, dem als erstem diese bewundernswerte Leistung 212 v. Chr. gelang, wurde ausgezeichnet durch den Zusatz "der Zweite nach Herakles“46. Diese fortlaufende Zählung der Heraklessieger endete mit dem siebten Sieger namens Nikostratos ${ }^{47}$ aus Kilikien, (Ol. 204=37 n. Chr.). Obwohl weitere Athleten diesen Titel hätten erlangen können, wurde dessen Vergabe von den Offiziellen von Olympia eingestellt. ${ }^{48}$

\footnotetext{
${ }^{37}$ Vgl. R. KNAB, Periodoniken, 14; K. KRAMER, Studien, 133; M. LÄMMER, Olympien, 32.

${ }^{38} \mathrm{Vgl}$. R. MERKELBACH, in:ZPE 18.2, 1975, $125 \mathrm{ff}$.

${ }^{39}$ Vgl. L. MORETTI, IAG, 189: „Col termine параסохомікєৎ si allude vagamente al carattere straordinario ed eccezionale di successi dell'atleta che si fregia die quel titulo."

${ }^{40}$ Vgl. $\square$ R. MERKELBACH, Über ein ephesisches Dekret für einen Athleten aus Aphrodisias

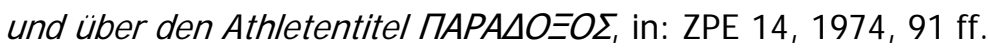

${ }^{41}$ Vgl. E. N. GARDI NER, $A A W, 105$; J. JÜTHNER, $A L$ 1, 129; QGAA s. v. Pankration, $187 \mathrm{f}$.

${ }^{42}$ L. MORETTI, IAG, 189; R. MERKELBACH, in: ZPE 14, 1974, $94 \mathrm{f}$.

${ }^{43}$ Vgl. L. FRIEDLÄNDER, SG2, 158 f.; E. N. GARDENER, GASF, 174; R. KNAB, Periodoniken, $13 \mathrm{f}$.

${ }^{44}$ Vgl. J. JÜTHNER, $A L 1,129$.

${ }^{45}$ PAUS. 5, 21, 10; L. MORETTI, Olympionikai, Nr. 587 f.;vgl. J. JÜTHNER, $A L$ 1, 129.

${ }^{46}$ Vgl. E. N. GARDI NER, AAW, 105.

${ }^{47}$ PAUS. 5, 21, 10; L. MORETTI, Olympionikai, Nr. $762 \mathrm{f}$.

${ }^{48}$.L. MORETTI, Olympionikai, Nr. 703.
} 
Athleten, die an einem Tag drei Konkurrenzen gewannen, durften sich mit dem Titel Dreifachsieger (трıабт́́) ${ }^{49}$ schmücken. Mit Siegen in den drei Kurzstrecken war dies schon 512 v. Chr. in Olympia dem Phanas aus Pellene $^{50}$ gelungen, was in der Folgezeit mehrere Athleten schafften ${ }^{51}$. Überragend in dieser Hinsicht war Leonidas von Rhodos, der bei vier Olympien hintereinander (164-152 v. Chr.) jeweils in den Laufdisziplinen Stadion, Diaulos und Waffenlauf siegte, was kein Zweiter mehr zustande brachte ${ }^{52}$. Eine Ausnahme stellte Polites von Keramos dar, der neben zwei Kurzstrecken auch den Dolichos gewann ${ }^{53}$. Durch seinen dreifachen Triumph bei den Isthmischen Spielen ging Kleistomachos von Theben als einziger Triastes der Schwerathletik bei einem bedeutenden Fest in die antike Sportgeschichte ein ${ }^{54}$.

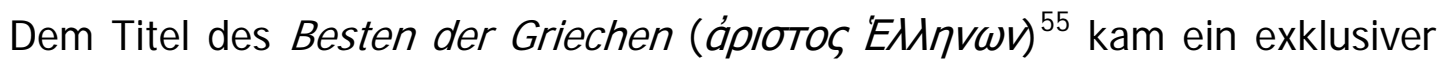
Anspruch zu. Er wurde ausschließlich den Siegern des extrem schwierigen Waffenlaufes von Plataiai verliehen ${ }^{56}$.

Auch im römisch geprägten Sportbetrieb kannte man zur Kenntlichmachung und Erhöhung der Leistung die Vergabe und Präsentation von Titeln: ${ }^{57}$

Im Gladiatorenwesen gab es verschiedene Klassifizierungen innerhalb der Kämpferschar, die auch die Form eines Titels annehmen ${ }^{58}$ konnten. Es gab

\footnotetext{
${ }^{49}$ Vgl. J. JÜTHNER, $A L$ 1, 128; H. SCHÖBEL, Olympia, 84.

${ }^{50} \mathrm{Vgl.} \mathrm{L.} \mathrm{MORETTI,} \mathrm{Olympionikai,} \mathrm{Nr.} \mathrm{142-144.}$

${ }^{51} \mathrm{Vgl.} \mathrm{J} \mathrm{.} \mathrm{JÜTHNER,} A L$ 1, $128 \mathrm{f}$.

${ }^{52} \mathrm{Vgl.}$ L. MORETTI, Olympionikai, Nr. 618-620; 622-624; 626-628; 633-635; vgl. J. JÜTHNER, AL 1, 129.

${ }^{53} \mathrm{Vgl.}$ L. MORETTI, Olympionikai, Nr. 796-798

${ }^{54}$ Vgl. L. MORETTI, Olympionikai, Nr. 584.

${ }^{55}$ Vgl. L. ROBERT, REA 31, 1929, 13 ff.; DERS., Hellenica 7, 1949, 80; R. KNAB, Periodoniken, 14; K. KRAMER, Studien, 52.

${ }^{56}$ Vgl. H. A. HARRIS, GAA, 75.

${ }^{57}$ Vgl. L. FRIEDLÄNDER, SG2, 54.
} 
eine Unterscheidung zwischen Anfängern (tirones) ${ }^{59}$ und wettkampferprobten Gladiatoren (veteranes) ${ }^{60}$. Zur Durchsetzung von Sparmaßnahmen kam es später zu einer Qualitätseinteilung der an einem munus Beteiligten. Die Gladiatoren wurden nach pekuniärer Wertschätzung in fünf Kategorien eingeteilt. Besonders interessant ist die Rangordnung innerhalb des Gladiatorenwesens, die man nach dem Übungspfahl palus bzw. пáł $\omega \varsigma$, der mit einer zusätlichen Zahlenangabe versehen war, bestimmte.

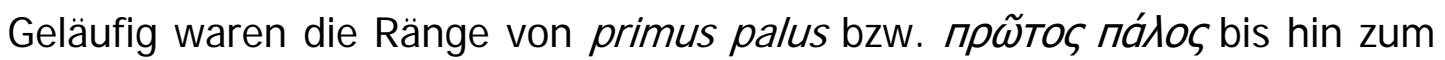
quartus palus bzw. те́тра пádo $\varsigma^{61}$. Da sich besonders erfolgreiche und

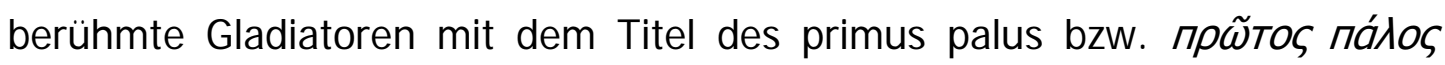
schmückten, wie etwa Kaiser Commodus ${ }^{62}$, kann man davon ausgehen, dass die Gladiatoren mit den Rängen zwei bis vier diesen nachgeordnet waren. Neuere archäologische Funde liefern Inschriften mit den Gladiatorenrängen von Palus fünf bis acht. Dieses wäre nicht verwunderlich, wenn nicht die häufig beigefügte Zahl an Kämpfen und/oder Siegen einen höheren Rang hätte erwarten lassen. Gründe dafür dürften in der erhöhten Zahl an Gladiatoren bzw. an munera gladiatoria gelegen haben.

Im römischen Pferdesport führten Wagenlenker, die tausend und mehr Siege herausgefahren hatten, den Titel eines miliarius ${ }^{63}$. Ebenso erhielten auch überdurchschnittlich erfolgreiche Rennpferde ${ }^{64}$ - über alle wurde exakt

\footnotetext{
${ }^{58}$ Vgl. Vgl. P. SABBATINI TUMOLESI, Gladiatorum, 33.

${ }^{59}$ Vgl. M. GRANT, Gladiators, 34.

${ }^{60}$ Vgl. M. J UNKELMANN, Spiel, 117.

${ }^{61}$ Vgl. L. ROBERT, Gladiateurs, $27 \mathrm{f}$.

62 Dio Cass., 72.

${ }^{63} \mathrm{Vgl}$. I. WEILER, Sport, $246 \mathrm{f}$.

${ }^{64}$ Vgl. G. BIANCO, in: RIL 111, 1977, 314 ff.
} 
Buch $^{65}$ geführt - bei hundert bzw. zweihundert oder mehr Erfolgen den Zusatz centenarius bzw. ducenarius ${ }^{66}$.

Für alle Titel galt, dass sie unabhängig von der Altersklasse und Geschlecht erzielt werden konnten. Im Speziellen unterschieden sie sich nach den Kriterien der Örtlichkeit, des Status des Festes, der Zahl der Erfolge, der Kombination der Siege, sowie außerordentlicher Wettkampfumstände. Die meisten Titel brachten nicht nur Ruhm und Ehre und finanziell-materielle Gewinne, sondern konnten auch mit Rechten und/oder Privilegien verbunden sein.

\footnotetext{
${ }^{65}$ Vgl. H. A. HARRIS, SGR, $203 \mathrm{f}$.

${ }^{66} \mathrm{Cl}$ 6, 10048; vgl. L. FRIEDLÄNDER, SG 2: „Auch die Dauerhaftigkeit berühmter Rennpferde war erstaunlich. Wenn der Renner Tuscus als Leitpferd des Fortunatus von der grünen Partei 386 mal, der Victor des Gutta Calpurnianus 429 mal siegte, so müssen sie nach allen überlieferten Zahlenverhältnissen wenigstens viermal so oft am Viergespann gerannt sein, also etwa 1600 bis 1700 mal, im großen Zrkus weit mehr als ebensoviel Meilen.“
} 


\section{7}

Rekorde

Der Wunsch der Menschen, sich mit Bestleistungen, sich mit Höchstwerten und Rekorden aller Art zu messen, sie einzustellen oder möglichst zu übertreffen, war ein allgemein weit verbreitetes Phänomen in der griechischrömischen Antike. ${ }^{1}$ Politiker ${ }^{2}$ und Militärs wollten an erster Stelle stehen, Männer aus Wirtschaft und Kultur strebten nach der Spitzenstellung, und Spielgeber ${ }^{3}$ wetteiferten um die Überbietung ihrer Vorgänger.

Von dem Wunsch geprägt, die Konkurrenz in irgendeiner Art und Weise als Ausnahmeerscheinung zu überragen, war der Wettkampfsport bei Griechen und Römern besonders geeignet.

Einige Autoren schreiben Leistungen unterschiedlichster (sportlicher) Art den Status des Rekordes zu. So wird schon im Titel von Beiträgen zum Weitsprung des Phayllus vom Rekordsprung geschrieben, ${ }^{4}$ die Laufleistung des Philonides wird als fantastischer Sportrekord gepriesen, ${ }^{5}$ und dass man

\footnotetext{
${ }^{1}$ Vgl. I. WEILER, AIEN APICTEYEIN, Ideologiekritische Bemerkungen zu einem vielzitierten Homerwort, in: Stadion 2, 1975, 199 ff., DERS., Wider und für das agonale Prinzip - eine griechische Eigenart?, in: Nikephoros 19, 2006, 81 ff. Vgl. M. KLOEREN, Sport und Rekord. Kultursoziologische Untersuchungen zum England des 16. bis 18. Jahrhunderts, Leipzig 1935 (ND Münster 1985)

${ }^{2}$ Vgl. P. VEYNE, August de l'an I. - Premier pontarque, in: BCH 90, 1966, 141 ff., bes. 146 f., n. 4.

${ }^{3}$ W. BEHRI NGER, Kulturgeschichte, 61: „Während Kaiser Augustus während seiner ganzen, 41 Jahre langen Regierungszeit etwa 3500 Tiere in die Kämpfe schickte, verbrauchte Kaiser Titus allein für die Einweihungsfeier des Kolosseums nicht weniger als 5000 Tiere. Kaiser Trajan ließ im J ahre 106 zur Feier seines Sieges über die Daker angeblich 11000 Tiere in das Amphitheater schicken, diese Mammutveranstaltung stellte den Höhepunkt der Sportart dar."

${ }^{4}$ Vgl.E. N. GARDENER, Phayllus and his Record Jump, in: JHS 24, 1904, 70-80; R. L. HOWLAND, Phayllus and the Long-Jump Record, Anth. Pal. Append. 297), in: PCPS 181, 1950-1951,30;

${ }^{5} \mathrm{Vgl}$. B. BILINSKI, in: Eos 50.1, 1959-1960, „... connu par son extraordinaire et presque fantastique record sportiv."
} 
beim Ringkampf nie auf die Knie gezwungen worden war, galt als Rekord. ${ }^{6}$

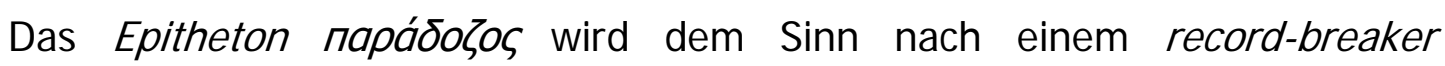
gleichgestellt, und auch der Titel трıaorís wird schlichtweg dem Rekordwesen zugeordnet. ${ }^{7}$

Die meisten uns überlieferten Rekorde finden wir auf bis zum heutigen Tag erhaltenen Originalen oder in zitierter Form beim Exegeten Pausanias. Auch die Verfasser von Siegeroden geizen nicht mit Superlativen. Manche Rekorde wurden von antiken Historikern und Annalisten erst nach Jahrhunderten eingefordert. Manche Bestleistung wird erst in neuerer Zeit als solche erkannt, erklärt und geehrt. Ein Weg zur Proklamation einer (vermeintlichen) Bestmarke war der Vermerk auf die kontinuierliche Folge von Siegen, was sowohl die Erfolge bei ein und demselben Fest bzw. der aufeinander folgenden Altersklassen wie auch Triumphe bei Festen, die man nacheinander im Rahmen seiner Wettkampftournee erzielt hatte. ${ }^{8}$ Seriensieger finden wir mehrfach bei den Läufern. So siegte ein Läufer aus Argos in Olympia viermal hintereinander im Diaulos der Männer, ${ }^{9}$ der berühmte Leonidas aus Rhodos war als ávń Olympionike im Stadion-, Doppel- und Waffenlauf. ${ }^{10}$ Nikoladas aus Korinth

\footnotetext{
${ }^{V}$ gl. M. I. FI NLEY/H. W. PLEKET, Spiele, 52: „Die Griechen [...] hatten andere Rekorde, [...] die Tatsache, daß er bei einem Ringkampf nie in die Knie gezwungen worden war. “

7 E. N. GARDENER, AAW, 106: „Paradoxos, ,Marvellous' or ,Record-Breaker', a title that suggests the advertisements of a music hall.“; F. BREIN, GL 2, 105: ,..., wenn wir von antiken Rekorden sprechen wollen ... ist der Ehrentitel Triastés überliefert.“.

8 J. EBERT, Epigramme, $93 \mathrm{f}$. : „Zwar lassen die in agonistischen Inschriften häufig

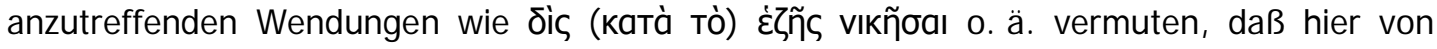
Erfolgen die Rede ist, die an mehreren, unmittelbar aufeinanderfolgenden Panathenäen erzielt worden sind, doch muß ein solcher Zusammenhang zwangsläufig dunkel bleiben, solange nicht eine zahlenmäßige Angabe der Siege erläuternd hinzutritt (z. B. тpiৎ

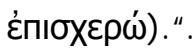

${ }^{9}$ Vgl. L. MORETTI, Olympionikai, Nr. 592; 595; 599; 605.

${ }^{10} \mathrm{Vgl.} \mathrm{L.} \mathrm{MORETTI,} \mathrm{Olympionikai,} \mathrm{Nr.} \mathrm{618-620,} \mathrm{622-624;} \mathrm{626-628;} \mathrm{633-635.}$
} 
siegte an fünf Panathenäen hintereinander, wobei zwei dieser fünf Siege als

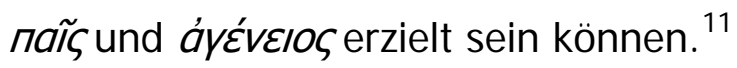

Dem Ansinnen, seiner Leistung den Charakter der Ausnahmeleistung

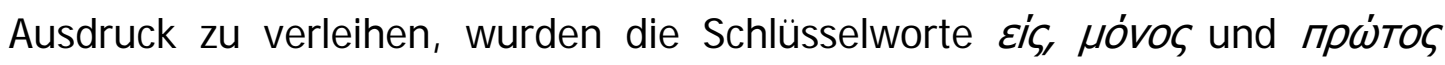
bzw. semel, unus und primus verwandt. ${ }^{12}$

Rekorde waren nicht nur aufgrund von Vergleichen mit anderen Leistungen möglich, sondern auch durch die Einmaligkeit der Leistung oder der Umstände. So führte Arrhichion an, der erste Athlet gewesen zu sein, der sich einen Trainer nahm bzw. sich einem wissenschaftlich fundierten Training unterwarf. ${ }^{13}$ Die Erstmaligkeit konnte auch beansprucht werden, wenn man bei einer Veranstaltung gesiegt hatte, die zum ersten Mal ausgetragen worden war, ${ }^{14}$ so wie der Periodonike T. Flavius Artemidoros aus Ardana in Kilikien der erste Pankrationsieger bei der Eröffnung der Kapitolinischen Spiele, die von Domitian im J ahre 86 n. Chr. eingeführt wurden, ${ }^{15}$ oder wenn es wie Fuscus, einem Wagenlenker der factio prasina, bei einer traditionellen Veranstaltung gelungen war, bei seiner ersten Teilnahme an einem Rennen auch gleich zu gewinnen. ${ }^{16}$ Die Einmaligkeit ihres Sieges mit dem Viergespann bei den Olympischen Spielen (nicht vor 360 v. Chr.) strich

\footnotetext{
${ }^{11} \mathrm{Vgl}$. J. EBERT, Epigramme, Nr. 26.

${ }^{12}$ M. N. TOD, in: CQ 43, 1949,110 f.: "The idea was usually expressed in very simple and

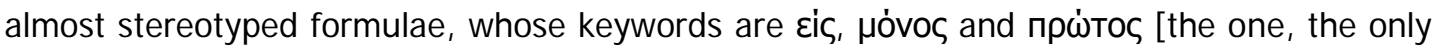
and the first], varied by combination and by the addition of phrases lending emphasis and expressiveness."

${ }^{13}$ Vgl. L. MORETTI, Olympionikai, Nr. 102.

${ }^{14}$ Dies betraf alle neuen Spielstiftungen, sowie alle Sportarten und -disziplinen, die im Laufe eines schon länger bestehenden Festes neu ins Programm aufgenommen wurden. Zur Aufnahme neuer Sportarten und -disziplinen in das Programm von Olympia vgl. W. DECKER, Sport, 36.

${ }^{15}$ Vgl. R. KNAB, Periodoniken, 40.

${ }^{16} \mathrm{CIL}$ 6, 33950; vgl. H. A. HARRIS, SGR, 206: „There is the record of Fuscus of the Green, who in $A D 35$ became the first man ever to win on his first day of racing."
} 
Kyniska, Tochter des Königs Archidamos II., aufgrund ihres Geschlechts heraus. ${ }^{17}$ Dromeus von Arkadien erzielte einen einmalig kuriosen Sieg: Sein Finalgegner Theogenes von Thasos konnte wegen Erschöpfung nicht antreten. ${ }^{18}$ Hieron von Syrakus stellte gleich bei einem Rennen zwei Bestmarken auf: Er ließ zusammen acht Gespanne von sich an den Start gehen und konnte drei der ersten vier Plätze belegen. ${ }^{19}$ Einen Sonderfall stellt der Doppelerfolg des Kapros dar. Er war der erste Athlet (при́то

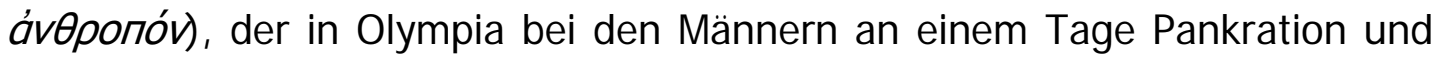
Ringkampf gewann. Er war somit der zweite nach dem Heroen Herakles, was somit nicht zu einer neuen Bestleistung, sondern lediglich zu deren Egalisierung führte. Gleiches galt für die inm folgenden Herakles-Sieger. ${ }^{20}$

Der Vermerk der Erst- und Einmaligkeit bezog sich meist auf die sportliche Leistung. Die mit Abstand wichtigste Informationsquelle bezüglich des Leistungsnachweises sind die Siegerepigramme, die vorab den Namen des Athleten, seines Vaters und der Heimatgemeinde nennen. Mit der Angabe von Ort und Charakter bzw. Wertigkeit des Festes wurde die erste Angabe zur Eingrenzung der Leistung gemacht, was sich erübrigen konnte, wenn die Inschrift am Austragungsort vorgefunden wurde. Der nächste Aspekt war die Angabe zur Sportart bzw. -disziplin, was sich auch erübrigen konnte, wenn die Inschrift sich an einer Statue befand, die den Sportler einer Sportart bzw. -disziplin eindeutig zuordnen ließ, wie etwa einen Boxer anhand seiner Faustwehr, einen Pankratiasten durch seine spezielle Kampfposition oder Technik und einen Pentathleten durch sein Sportgerät (z. B. Halteren, Speer,

${ }^{17}$ J. EBERT, Epigramme, Nr. 33 ; vgl. H. A. HARRIS, SGR, 178; D. C. YOUNG, in: Nikephoros 9, 1996, 181: „Inscription states a gender record: the only woman to the Olympic chariot contest." Nach W. DECKER, Nil, 79, hat Berenike I durch den Verweis auf die Erst-und Einmaligkeit ihres Triumphes den alten Ruhm Kyniskas in Sparta ausgelöscht.

${ }^{18}$ Paus. 6. 6. 5 f.; L. MORETTI, Olympionikai, Nr. 202; vgl. D. C. YOUNG, in: Nikephoros 9, 1996, 182.

${ }^{19}$ Bacchylides 3, 15; vgl. D. C. YOUNG, in: Nikephoros 9, 1996, 182, n. 22.

${ }^{20}$ Paus. 5, 21, 10; L. MORETTI, Olympionikai, Nr. 587-588. 
Diskus). Gleiches galt auch für die Einordnung in eine der Altersklassen anhand von Körperbau bzw. Barttracht. ${ }^{21}$ So waren es in erster Linie die geographischen Angaben, die eine Inanspruchnahme eines Rekords in Form der Erst- und/oder Einmaligkeit möglich machten. So hatte Mykon, des Taureas Sohn, als einziger der Ionier bei den Pythien im Boxen der Knaben gesiegt. ${ }^{22}$ Agias war der erste aus Tessalien, der in Olympia im Pankration der Männer gewann. ${ }^{23}$ Xenombrotos, Sohn des Xenodikos, war der erste Koer, der mit dem Reitpferd Pisas Siegespreis erringen konnte. ${ }^{24}$ Für die Inanspruchnahme der Erst- und Einmaligkeit waren Sportart und -disziplin sowie Altersklasse - beim Pferdesport spielte die Altersklasse keine Rolle nicht von Bedeutung. Es wurde auch die tautologische Formel des $\mu$ óvo $\mathrm{kaI}$ прஸ́тоৎ gewählt. Relativierend wirkt dann die Reduktion der Inanspruchnahme der Erst- und/oder Einmaligkeit einer Leistung, wenn man sie im Kontext mit den weiteren Informationen betrachtet.

Rekorde konnte man auch nach bestimmter Konstellation der Siege einfordern. In Delphi befanden sich die Statuen von drei Brüdern, die alle bei den Isthmischen Spielen - jeweils in Pankration, Ringen und Stadionlauf ${ }^{25}$ erfolgreich gewesen waren, was in dieser Zusammenstellung einmalig war. ${ }^{26}$ Besondere Umstände eines Sieges konnten auch dazu verleiten, sei es für die Kommentatoren, sei es für die Sportler selbst, als Novität ein einmaliges

\footnotetext{
${ }^{21} \mathrm{Vgl}$. J. EBERT, Epigramme, $18 \mathrm{f}$.

${ }^{22} \mathrm{Vgl}$. J. EBERT, Epigramme, 31.

${ }^{23}$ Vgl. L. MORETTI, Olympionikai, Nr. 192; J. EBERT, Epigramme, Nr. 43; vgl. D. C. YOUNG, in: Nikephoros 9, 1996, 180.

${ }^{24}$ Paus. 6, 14, 12; J. EBERT, Epigramme, Nr. 49.

${ }^{25} \mathrm{Vgl}$. J. EBERT, Epigramme, Nr. 43; Nr. 44; Nr. 45.

${ }^{26}$ Vgl. D. C. YOUNG, in: Nikephoros 9, 1996, $180 \mathrm{f}$. Zu einem dynastischen Rekord im Pferdesport; W. DECKER, Nil, $84 \mathrm{f:}$ „Ptolemaios II, der hier spricht, nennt einleitend die olympischen Siege im Viergespann, die sein Vater Ptolmaios I., seine Mutter Berenike I. und er errungen haben in doppelter Qualität, ,erstmalig und einzigartig'. Diese Konstellation kann den drei Königen niemand mehr streitig machen. Sie bildet einen Rekord, wie er typisch ist für griechisches Empfinden.“
} 
Ereignis herauszustreichen. Der lusitanische Wagenlenker Diocles siegte an einem Tag gleich zweimal mit dem Sechsgespann zu je 60.000 Sesterzen, sowie er einmal mit dem Siebengespann gewann und das ganze ohne Zuhilfenahme der Peitsche. ${ }^{27}$ Eine rekordverdächtige Gewinnsumme von fünfzehn Sack Gold soll laut Martial der Wagenlenker Scorpus in einer Stunde verdient haben. ${ }^{28}$ Eine außerordentliche, rekordgleiche Leistung wird dem Laufolympioniken Polites von Karien zugeschrieben: Aufgrund seiner Vielseitigkeit gewann er neben zwei Kurzstreckendisziplinen auch den ,dolichos', was noch keinem vorher gelungen war. ${ }^{29}$

In der Inschrift eines uns unbekannten Milesiers ${ }^{30}$ finden wir ein geradezu übersteigertes Bedürfnis ${ }^{31}$, die Erfolge mit dem Prädikat der Erst- bzw. Erstund Einmaligkeit zu erhöhen.

Dieser zweifelsfrei außergewöhnlich erfolgreiche Läufer - u. a. zwei Mal als

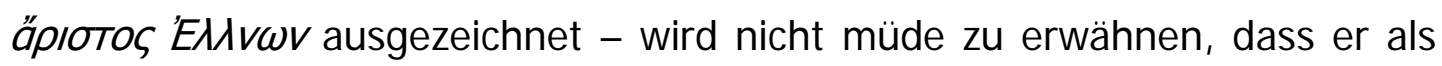

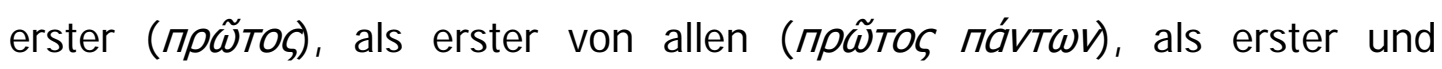

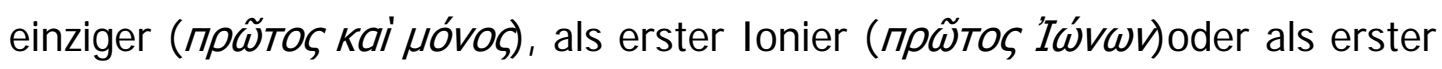

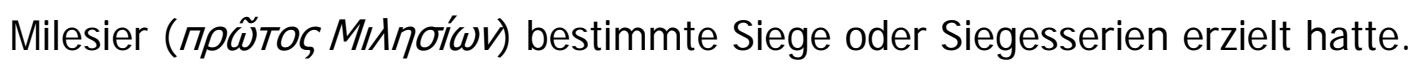
Als Beispiel für eine Inschrift mit einer ganzen Anzahl rühmender und viel diskutierter Epitheta $^{32}$ kann die hochinteressante ${ }^{33}$ Inschrift des

${ }^{27}$ CIL 6, 10048=ILS 5287; J.-P. THUI LLIER, Rom, 105: „Diocles spricht selbst von neuen Gespannformen (novae coactiones) und präzisiert dahingehend, daß ein Gespann mit sieben Pferden noch nie zu sehen war."

${ }^{28}$ Martial 10, 74, 5: „Cum Scorpus una quindecim graves hora ferventis auri victor auferat saccos."

${ }^{29}$ Vgl. D. C. YOUNG, in: Nikephoros 9, 1996, 177: „Polites' diversity at running seemed

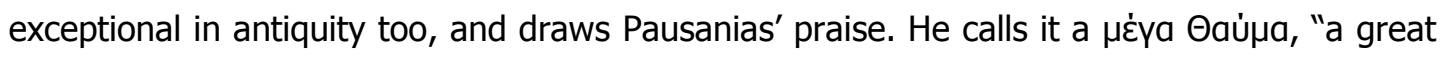
marvel".".

${ }^{30}$ Vgl. L. MORETTI, IAG, Nr. 59.

${ }^{31}$ H. A. HARRIS, GAA, 126: "There is an inscription of the first century A. D. about a runner from Miletus, whose name is losts, which shows an amusing anxiety to establish some category of priority for all his successes." 
Schwerathleten M. Aurelius Asklepiades gesehen werden ${ }^{34}$. Denn diese komplett erhaltene Inschrift wird gern als Beispiel für die Zurschaustellung von Leistung und Eigenwerbung, mehr noch, als Beleg für die Vorliebe antiker Athleten, Rekorde zu brechen, präsentiert. ${ }^{35}$ So erfahren wir von dem als Pankratiasten unbesiegten Periodoniken ${ }^{36}$, dass er nie unentschieden gekämpft habe, dass er nie selbst einen Gegner herausgefordert habe, noch jemand es gewagt habe, ihn herauszufordern, dass er nie einen Kampf durch kaiserliche Gunst gewonnen habe, dass er alle Wettkämpfe, zu denen er sich gemeldet hatte, als Sieger abschloss u. v. . $^{37}$

Ein schneller und relativ einfacher Weg, einen Rekord für sich zu beanspruchen, war die Kombination von Siegen, die es in dieser Form zuvor noch nicht gegeben hatte. Geradezu ein Paket von Rekorden ${ }^{38}$ bietet uns die Inschrift des Schwerathleten Theogenes von Thasos. ${ }^{39}$ Dieser versucht in

${ }^{32}$ Vgl. M. POLIAKOFF, Kampfsport, 245, n. 21: „...die von Asklepiades gebrauchten Ausdrücke haben zu erheblichen Meinungsverschiedenheiten Anlaß gegeben."

${ }^{33} \mathrm{Vgl}$. L. MORETTI, IAG, 230: "Questo è certo la più interessante tra tutti le iscrizioni agonistiche, non tanto per la lunghezza o il numero delle vittorie o degli agonic he vi sono redordati (quasi tutti già noti), quanto per l'abbondanza delle espressioni proprie del mondo degli atleti e, se si vuole, perchè sembra, più che in ogni altro documento del genere, scorgere, oltre che l'atleta, l'uomo.“

${ }^{34}$ IG 14,1102; L. MORETTI, IAG, Nr. 79; vgl. H. A. HARRIS, GAA, 127 f. W. DECKER, Sport, 115 ff.; DERS., Olympiasieger aus Ägypten, in: E. GRAEFE/U. VERHOVEN (Hrsg.), Religion und Philosophie im Alten Ägypten. Festgabe für Philippe DERCHAIN zu seinem 65. Geburtstag am 24. Juli 1991, Leuven 1991, 93-105.

${ }^{35} \mathrm{Vgl}$. E. N. GARDENER, $A A W, 112$ : „The spirit of ostentaion and advertisement, and the love of record-breaking ... in the inscription on ... Asclepiades of Alexandria."

${ }^{36}$ Vgl. E. N. GARDENER, $A A W, 112:$ “... follows the description of his unbeaten record as a 'pancratiast invincible, immovable, univalled'."

${ }^{37}$ Zu einer hier geänderten Anordnung der Aspekte vgl. auch W. DECKER, Sport, 115.

${ }^{38}$ Vgl. D. C. YOUNG, in: Nikephoros 9, 1996, 183.

${ }^{39}$ Vgl. L. MORETTI, IAG, Nr. 21; J. EBERT, Epigramme, Nr. 37; W. DECKER, Sport, 125 ff.; DERS., in: DNP 12, 2001, 350, s. v. Theogenes (1). 
vielfältiger Form Rekorde darzustellen, ${ }^{40}$ geflissentlich nach Wertigkeit der Austragungsstätte aufgereiht, gipfelt sein Katalog mit den Bestmarken seiner Lebensleistung: ${ }^{41}$

Seinen fest angestrebten Doppelsieg im Faustkampf und Pankration hatte noch kein anderer Sterblicher je geschafft. Noch nie wurde in Olympia je ein und derselbe Mann bekränzt als Sieger sowohl im Faustkampf als auch im Pankration. ${ }^{42}$ Bei den pythischen Spielen gelang inm, was keinem Mann zuvor vergönnt war: Er gewann alle drei schwerathletischen Konkurrenzen, und von diesen drei Kränzen gewann er einen kampflos. ${ }^{43}$

Es folgte sein isthmischer Rekord ${ }^{44}$ mit zehn Siegen bei der Teilnahme an neun Festen. Sein Doppelsieg im Faustkampf und Pankration wurde vom Herold als Weltrekord ${ }^{45}$ verkündet. Der Reigen an Superlativen in der Feststellung der Gesamtzahl seiner Triumphe: Theogenes von Thasos hatte 1.300 Siege ${ }^{46}$ vorzuweisen. Solch eine hohe Zahl an Siegen scheint bei einem solch außergewöhnlichen und vielseitigen Spitzensportler $^{47}$ als absolut realistisch akzeptiert zu werden, zumal seine aktive Teilnahme am

\footnotetext{
${ }^{40}$ Vgl. D. C. YOUNG, in: Nikephoros 9, 1996, 183: "Theogenes' inscription provides, in fact, a model for the vocabulary of Greek record-breaking."

${ }^{41}$ Vgl. L. MORETTI, IAG, 51; J. EBERT, Epigramme, 119.

42 D. C. YOUNG, in: Nikephoros 9, 1996, 183: „That victory (in Pankration, d. Verf.), coupled to his earlier boxing crown, made him the first man ever to combine victories in both those Olympic combative events. He thus set a new record."

${ }^{43}$ Vgl. L. MORETTI, IAG, 51; J. EBERT, Epigramme, 120; D. C. YOUNG, in: Nikephoros 9, 1996, 183: „That Olympic record naturally comes first. Next comes Theogenes' record at the Pythians at Delphi."

${ }^{44}$ Vgl. L. MORETTI, IAG, Nr. 21; J. EBERT, Epigramme, Nr. 37.

45 D. C. YOUNG, in: Nikephoros 9, 1996, 183: „For twice the herald proclaimed him world record holder, the 'only man on earth to win both boxing and no-holds-barred in a single day'."

${ }^{46}$ Paus. 6, 11, 5 führt 1.400 Siege an; nach Plut. Praec. Publ. ger. 15,7 sind es 1.200 Siege.

47 J. EBERT, Epigramme, S. 120: ,..., und somit ist die Vermutung gestattet, daß sich Theogenes - vielleicht in seiner Jugendzeit - in allen schwerathletischen Disziplinen und auch in mehreren Laufkonkurrenzen mit Erfolg versucht hat."
} 
Wettkampfgeschehen $22 \mathrm{~J} \mathrm{ahre}^{48}$ dauerte, und somit auch rekordverdächtig erscheint. Schließlich und endlich wird mit Nachdruck darauf verwiesen, dass er in seiner gesamten Karriere im Faustkampf unbezwungen blieb. ${ }^{49}$

Nahezu in die Nähe einer übersteigerten Suche nach Rekorden ${ }^{50}$ kann man die Aussagen und konstruierten Vergleiche in den Inschriften römischer - wie später byzantinischer ${ }^{51}$ - Wagenlenker rücken. Die Inschrift des lusitanischen ${ }^{52}$ Wagenlenkers Gaius Apuleius Diocles ${ }^{53}$ diente uns beispielhaft für die gezielte Suche nach (künstlich konstruierten) Rekorden ${ }^{54}$. Er verglich seine Leistungen und Erfolge mit anderen, wo immer sie zu seinen Gunsten ausfielen. Diese Vergleiche konnten mit einzelnen Lenkern seiner Partei oder einer anderen factio oder gleich mehreren Fahrern (verschiedener Parteien) zusammen angestellt werden. Bei einer vierundzwanzigjährigen Karriere als 48 D. C. YOUNG, Myth, 150: "Theogenes was the Olympic, Pythian and Isthmian record holder, who won more than a thousand athletic victories in his twenty-two year career."

${ }^{49}$ W. DECKER, Sport, 68: „Kaum weniger eindrucksvoll ist der Rekord des Theogenes von der Insel Thasos der nach einer in Delphi aufgestellten Inschrift während 22 Jahren im Faustkampf ohne Niederlage blieb.“

${ }^{50}$ Vgl. D. RAMBA, Recordmania, 34 f.; J.-P. THUILLIER, Rom, 130: „Aber primus zu sein, erster in seiner Kategorie, welche es auch immer sein moche, war bei den Römern (wie in einem wohl noch höheren Maße bei den Griechen) ein existenzielles Ziel, dessen zu rühmen man im Kreise seinesgleichen nicht müde wurde.“

${ }^{51}$ Zu den Rekorden byzantinischer Wagenlenker vgl. A. CAMERON, Porphyrius, 168 (Uranius, der als einziger mit einer Goldstatue geehrt wurde) und 206 ff. (Porphyrius, der als einziger schon zu Lebzeiten eine Statue bekam).

52 J.-P. THUILLIER, Rom, 132: „Der überaus berühmte Diocles (eminentissimus omnium agitatorum-das muß kaum übersetzt warden) verkündet, er sei Lusitaner, was nicht verwundert, ist doch die Iberische Halbinsel als das Eldorado der Rennpferde bekannt."

53 J.-P. THUILLIER, Rom, 109, spricht bei dem Wagenlenker Diocles von einem "unbestrittenen Superstar".

${ }^{54} \mathrm{ClL}$ 6, 10048=ILS 5287; W. DECKER, in: A. CARTER/A. KRÜGER, Record, 21 f.: "They (A. KRÜGER/A. ITO, d. Verf.) have translated the inscription on the tombstone of C. Apuleius Diocles, which records the unsurpassed career of a charioteer in the Roman Circus. The inscription lists all his victories in competition held over nearly twenty-four years; in modern terms, this can only be called a record list." 
herausragenden Lenker eröffneten sich ihm anhand einer stattlichen Basis an Erfolgen unterschiedlichster Art eine Vielzahl an Möglichkeiten des Leistungsvergleichs. Aspekte der Vergleiche, die inn wiederholt als Rekordmann dastehen ließen, waren - in Kombination mit der Anzahl der Siege - u. a. die Triumphe bei Rennen mit außerordentlichen Siegprämien, die Art und Weise des Sieges und die Erfolge mit Pferden bestimmter Herkunft. Er verglich sich mit einem Lenker der grünen Partei, der als erster seit Gründung der Stadt die außerordentliche Gewinnsumme von 50.000 Sesterzen sieben Mal gewann. Diocles gewann diese praemia maiora acht Mal. ${ }^{55}$ Diocles verglich sich mit Fortunatus von der factio prasina, der mit seinem Renner Tuscus die Gewinnsumme zu 50.000 Sesterzen neun Mal einstreichen konnte. Er aber gewann diese Summe zehn Mal und dazu ein Rennen zu 60.000 Sesterzen mit dem Pferd Pompeianus. Sein Zeitgenosse Pontius Epaphroditus war zwar erfolgreicher als er (1.467 zu 1.462 Siege), aber er übertraf inn in den Rennen eripuit et vicit (467 zu 502), die das höchste Ansehen genossen. ${ }^{56}$ Diocles rühmte sich, der erfolgreichste Wagenlenker mit Rennpferden aus Afrika zu sein. Er übertraf die bis dahin besten aurigae mit afrikanischen Pferden - Epaphroditus und Musclosus allein mit seinem Ausnahmerenner Pompeianus und dessen 152 Siegen. Bei einer anderen Gelegenheit verglich sich Diocles mit drei besonders berühmten und gefeierten aurigae verschiedener Parteien. Diese drei Lenker - unter innen der von Martial gepriesene Scorpus - hatten zusammen mehr als vier Mal so viele Siege wie Diocles allein und brachten es gemeinsam auf achtundzwanzig Siege in Rennen mit der stattlichen Gewinnsumme von 50.000 Sesterzen. Diocles aber strich allein diesen Betrag neunundzwanzig Mal ein; er war der Meister, was Rennen mit außerordentlichen Siegprämien anbelangte. ${ }^{57}$ Diocles wurde nicht müde, weitere Rekorde und Titel (novis coactionibus et numquam ante titulis scriptis Diocles eminet) für sich

${ }^{55} \mathrm{CIL}$ 6,10048 ;vgl. F. DREXEL, Inschriften, in: SG 4, 194, H. A. HARRIS, SGR, 199: "Diocles gives statistics about prize money."

${ }^{56}$ CIL 6,10048; vgl.H. A. HARRIS, SGR, 199.

${ }^{57}$ Vgl. H. A. HARRIS, SGR, 200. 
einzufordern. So startete er an einem Tag zweimal mit dem Sechsgespann und gewann beide Rennen zu je 60.000 Sesterzen, oder er gewann ein Rennen mit dem Siebengespann (septem equis in se iunctis), ohne die Peitsche zu benutzen (sine flagello). Diocles brach Rekorde von Wagenlenkern seiner eigenen factio wie auch die von agitatores anderer Parteien und setzte gleichzeitig neue Maßstäbe für alle.

Es ist nicht von der Hand zu weisen, dass einige angeführte prägende Merkmale des antiken Sports den Rekord gefördert und erst ermöglicht haben. Professionalismus und Leistungsmessung, Zahlenwelt und Titel, all dieses erschien gebündelt im Rekordwesen. Der Rekord stellte somit eine nahezu geniale Abstraktion eines Leistungsvergleichs zwischen Lebenden und Toten und in gewisser Weise eine einzigartige Form der Unsterblichkeit dar. ${ }^{58}$

Wir haben feststellen können, dass es für Sportler in der griechischrömischen Antike nicht nur wichtig war zu gewinnen, sondern, dass sie auch Wert darauf legten, eine außergewöhnliche Leistung zu erbringen, die möglichst vorherig erzielte Bestmarken überbot.

Daher ist es nicht nachvollziehbar, ${ }^{59}$ dass einige Wissenschaftler den Rekord für eine Erfindung des demokratischen Industriezeitalters halten, ${ }^{60}$ und sogar

\footnotetext{
${ }^{58}$ A. GUTTMANN, Ritual, $51 \mathrm{f}$. ."It is the marvelous abstraction that permits competition not only among those gathered together on the field of sport but also among them and others distant in time and space." Vgl. ebd., 55 ("uniquely modern form of immortality").

${ }^{59}$ Vgl. W. DECKER, Der Rekord des Rituals, in: G. SPITZER/D. SCHMIDT (Hrsg.), Sport zwischen Eigenständigkeit und Selbstbestimmung: Pädagogische und historische Beiträge aus der Sportwissenschaft, FS für HAJO BERNETT, Bonn 1986, 66: „Bei allem Respekt vor der Brillanz, Belesenheit und analytischen Schärfe der Anhänger der These vom neuzeitlichen Entstehen des Rekordbegriffes hätte man sich gewünscht, daß diese sich mehr als geschehen durch eine profunde Betrachtung der antiken Verhältnisse abgesichert hätten."

${ }^{60} \mathrm{Vgl}$. R. D. MANDELL, The Invention of the Sport Record, in: Stadion 2, 1976, 250-264.
} 
die Existenz des Rekordes bzw. das Streben nach Rekorden der grieschischrömischen Antike absprechen. ${ }^{61}$

${ }^{61}$ Vgl. A. GUTTMANN, Ritual, 54 ("The Characteristics of Sports in Various Ages"). 
Zum Abschluss sollen noch einmal alle sieben Charakteristika, die den Sport der griechisch-römischen Antike entscheidend geprägt haben, in komprimierter Form herausgestellt werden.

Seit archaischer Zeit waren Sportveranstaltungen zur Unterhaltung der griechisch-römischen Bevölkerung der Antike ein elementarer Bestandteil des öffentlichen Lebens. An bedeutenden Kultstätten (z. B. Olympia) wie in profanen Prachtbauten (z. B. Circus Maximus), die enorme Zuschauermengen aufnehmen konnten, wurde für vielseitige Unterhaltung gesorgt. Es waren die sportlichen Wettkämpfe auf höchstsem Niveau, die neben einem abwechlungsreichen Beiprogramm bzw. Umfeld -für hohe Anziehungskraft sorgten.und ein prägendes Moment der antiken Gesellschaft darstellten.

Die Protagonisten, die Wettkämpfer, konnten nicht nur durch gute Leistungen und Erfolge Ruhm und Ehre sowie finanzielle/materielle Gewinne erzielen, sondern vor aller Welt gleichzeitig Werbung für sich und die eigene Heimatgemeinde (z. B. Pferdezucht) machen. Für aufstrebende Politiker wie für Amtsinhaber, aber auch für unumstrittene Herrscher, boten die Veranstaltungen mannigfaltige Möglichkeiten der Kontaktaufnahme und Selbstdarstellung. Von (demagogischen) Reden über (z. T. verbotene) Zurschaustellung ihrer Großzügigkeit, von der Demonstration der Macht bis zur Präsentation potentieller Nachfolger war ein vielfältiges Spektrum politischer Aktivitäten möglich. Das überweigend sachkundige Publikum nutzte die Gelegenheit, nicht nur zu den sportlichen Darbietungen seine Meinung kundzutun. Von Zustimmung und Anerkennung bis zu harscher Kritik und massiven Forderungen - die sowohl politischen wie ökonomischen und privaten Charakter haben konnten - reichten die öffentlichen Demonstrationen, die schon für einen Ersatz von Volksversammlungen gesehen werden konnten. 
Der Wettkampfsport bei Griechen und Römern war von Anfang an eine ernste und strapazenreiche Tätigkeit. Eine Trennung von Amateueren und Professionals kannte man nicht. Allein Können und Leistung entschieden über den Sieg.

Der Sieg, der erste Platz, zählte, man wollte der Beste sein. Diesem Streben nach höchsten sportlichen Ehren wurde das persönliche Leben unterworfen und das gesamte Umfeld danach ausgerichtet. Unter der Anleitung von professionellen Trainern wurde nach ausgeklügelten Trainingsplänen bzw. zyklen geübt. Aufbau und Verbesserung von Physis und Technik standen im Vordergrund, aber auch taktische und psychologische Aspekte flossen in die Trainingsarbeit ein. Auch die spezielle Ernährung sowie medizinische und physiotherapeutische Betreuung verliehen dem Sport der griechischrömischen Antike sportwissenschaftliche Züge.

Sportliche Erfolge brachten dem siegreichen Athleten - wie auch seinem Trainer, seiner Familie und der Heimatgemeinde - Ehre und Anerkennung. Für den Sportler selbst war ein siegreiches Abschneiden bei bedeutenden Veranstaltungen meist mit lukrativen finanziellen/materiellen Gewinnen verbunden. Ferner ging oft ein sozialer Aufstieg und eine Anzahl von Vergünstigungen und Annehmlichkeiten damit einher.

Die meisten Athleten waren in Verbänden organisiert, die sich sowohl um die Ausrichtung der Veranstaltungen kümmerten als auch die Interessen ihrer Mitglieder wahrnahmen.

Von den meisten Sportlern wissen wir, dass sie mit ihren Kameraden gemeinsam lebten und trainierten und trotz manch überlieferter Skandale fair miteinander umgingen.

Der Wettkampfsport der griechisch-römischen Antike war in einem sehr hohen Maße vom Streben nach größtmöglicher Chancengleichheit geprägt. Für die Wettkämpfe galt allgemein eine Einteilung der Sportler (und Tiere) in 
Altersklassen. Das gesamte Wettkampfwesen wurde von einem genau vorgegebenen Regelwerk geordnet. Die Leitung der Kämpfe und Kontrolle bezüglich der Einhaltung der Wettkampfregeln oblag gut geschulten und hoch angesehenen Schiedsrichtern. Für die technischen Disziplinen galt, dass an dem bestimmten Austragungsort alle Teilnehmer dieselben Sportgeräte benutzten. Bei den Gladiatorenkämpfen kannte man Gefechte zwischen Vertretern derselben Kämpfergattung und solchen, bei denen die Kontrahenten völlig unterschiedlich gerüstet und bewaffnet waren, wobei sich die Vor- und Nachteile des einen mit den Vor- und Nachteilen des anderen regelrecht neutralisierten. Für die Laufkonkurrenzen nutzte man ein System von Ablaufschranken, deren Zweck vor allem darin bestand, Frühstarts zu verhindern. Dem gleichen Zweck dienten auch die ausgeklügelten Startanlagen bei den Wagenrennen. Von der Athletik über die Gladiatorenkämpfe bis hin zu den hippischen Agonen und Wagenrennen wurde im gesamten Wettkampfsport - ganz in Gegensatz zu einer eklatanten Chancenungleichheit im gesellschaftlichen Leben- ein ausgesprochen hoher Stellenwert beigemessen.

Die antike Gesellschaft war in allen Bereichen stark auf Leistung fixiert. Stets und überall wurde von allen Mitgliedern Leistungen, die auf unterschiedliche Art und Weise gemessen, bewertet und verglichen werden konnten, erwartet und gefordert. Der Sport wurde als Vorbild für dieses Leistungsprinzip gesehen. Hier spielten jedoch die Sportarten und -disziplinen, bei denen sich die Leistung in Weite, Gewicht oder Zeit bestimmen ließ, eine untergeordnete Rolle, wobei auch hier rekordverdächtige Bestmarken ihre Aufmerksamkeit fanden.

Bei den technischen Disziplinen Weitsprung, Speer- und Diskuswurf wurden die erzielten Weiten erfasst, spielten aber für die Siegerermittlung keine Rolle. Für die Kurzstreckenläufe sind mangels technischer Möglichkeiten keine Zeiten überliefert, lediglich von Läufen über größere Distanzen kennen wir Strecke und Zeit, was Rückschlüsse auf die Leistung zulässt. 
Eine andere Form der Leistungsmessung im Sport war die Wertung eines Sieges hinsichtlich seiner Qualität, was vor allem in der Schwerathletik zur Geltung kam. Hier kannte man je nach Sportart und -disziplin verschiedene Formen des Ausdrucks zur Aufwertung eines schon beachtlichen Erfolges. Manche dieser Epitheta galten nur für Ringer, andere für Faustkämpfer, und allgemein wurde ein kampfloser Sieg besonders hoch geschätzt.

Zahlen hatten für die Welt der griechisch-römischen Antike eine immense Bedeutung. Sie waren nahezu in allen Bereichen der antiken Welt präsent und oft prägend. Besonders im Bereich des Sports hatte der Umgang mit Zahlen und Statistiken einen enorm hohen Stellenwert. Fast alle Mitteilungen über sportliche Erfolge waren mit Zahlenangaben verbunden. Wo immer es möglich erschien, wurde die Übertragung von der Qualität einer sportlichen Leistung in die Quantität vollzogen. Die Auflistungen begannen mit Zahlenangaben zu Erfolgen bei den großen Festen, gefolgt von Siegen bei weniger bedeutenden Veranstaltungen, konnten sich aber auch auf Heimsiege oder Triumphe in anderen ,Erdteilen' beziehen. Siege konnten auch zahlenmäßig zusammengefasst werden mit Bezug auf den Trainer, Geschwister oder das Elternhaus. Man kannte auch gemischte Auflistungen, wie wir sie bei den Gladiatoren finden, wo neben der Zahl der Kämpfe, Siege und Unentschieden, auch die Zahl der Niederlagen aufgelistet waren, die keine (tödlichen) Konsequenzen für den Besiegten zur Folge gehabt hatten. Gelegentlich wurden von den Verfassern der Siegverzeichnisse die Betrachter zum Zählen aufgefordert. Gerne wurden Aussagen gemacht, die zahlenmäßig nicht konkret fassbar waren, aber scheinbar auf eine erwünschte, besonders hohe Summe schließen lassen sollten. So finden sich Bemerkungen wie etwa, dass die Siege so zahlreich waren wie Kiesel am Strand, oder man so viele Triumphe hatte feiern können, für die der Platz nicht reiche alle aufzulisten. Eine ausgeprägte Vorliebe für tabellarische Erfolgsnachweise finden wir in den Inschriften römischer Wagenlenker. Zuerst führten sie die Zahl ihrer Starts, Siege und Platzierungen an. Diese Angaben konnten aufgeschlüsselt 
sein nach Parteien (factiones), für die sie gefahren waren. Ferner konnte eine Unterteilung hinsichtlich der Zahl der am Rennen beteiligten Gespanne (z. B. certamen singularum) erfolgen. Ein weiterer Aspekt der Zahlenangaben konnte sich auf die Höhe der Gewinnsummen beziehen, wobei Siege in außerordentlich hoch dotierten Rennen gesondert vermerkt wurden. Auch die Aufschlüsselung der Erfolge mit bestimmten Pferden fand seinen Niederschlag in den Statistiken. Man kann sagen, dass der antike Sport ein breites Feld für zahlenmäßige Erfassung und Wiedergabe von Leistungen und Erfolgen bereithielt, das von schlichtem Zahlwerk über umfassende Statistiken verschiedener Art bis hin zu Zahlenspielereien (z.B.Namensgebung des Sohnes nach Zahl der eigenen Olympiasiege)alles bereit hielt.

Der Wettkampfsport implizierte für die Teilnehmer das Streben nach Sieg und vor allem nach Titeln. Diese konnten finanzielle/materielle Gewinne mit sich bringen, wie auch mit Rechten und Privilegien verbunden sein. Manche Titel galten nur für bestimmte Feste bzw. Austragungsorte, andere hatten Allgemeingültigkeit. Die Sieger bei den Olympischen Spielen oder den Festen zu Delphi nannten sich Olympioniken bzw. Pythioniken, also entsprechend des Festortes oder der dort verehrten Gottheit. Gleiches galt für die anderen Periodosspiele. Für Sieger des gesamten Zyklus kannte man den Titel des Periodoniken. Einzigartig war der Titel des aristos Hellenon, der ausschließlich den Siegern des äußerst schwierigen Waffenlaufes von Plataiai verliehen wurde. Allgemeingültigkeit hatten Titel wie die des Hieroniken (Sieger in Heiligen Spielen), Pleistoniken (Sieger bei zahlreichen Spielen), Paradoxoniken (Doppelsieger) und Triastes (Dreifachsieger). Die Titelvergabe war unabhängig von Sportart und -disziplin und galt auch für alle Altersklassen.

Im Gladiatorenwesen kannte man eine Trennung von Anfängern (tirones) und Altgedienten (veteranes). Innerhalb der erprobten Kämpfer galt eine 
Einteilung, die wie der Übungspfahl náłos/palus benannt wurde und mit Zahlzeichen versehen eine Unterteilung vom ersten (wie etwa Kaiser Commodus) bis achten Rang kannte.

Bei den Wagenlenkern unterschied man zwischen Lenkern, die mit dem Zweigespann (biga) oder dem Dreigespann ( triga) fuhren, die aurigae hießen und den agitatores, die ihre Rennen mit Vier- und Mehrgespannen (quadriga etc.) bestritten. Wagenlenker, die tausend und mehr Siege herausgefahren hatten, schmückten sich mit dem Titel eines miliarius. Pferde, die an hundert bzw. zweihundert Siegen beteiligt gewesen waren, nannte man centenarii bzw. ducenarii.

Von großem Interesse war es für den/die antike/n Wettkampfsportler/in, sich in irgendeiner Weise als Rekordmann/-frau präsentieren zu können. Meist wurde dies durch die Anbgbe der Erst- und/oder Einmaligkeit seines/ihres Triumphes gemacht. Nachdem durch Angbe des Austragungsortes, der Disziplin und der Altersklasse der Erfolg schon eingegrenzt wurde, reduzierte man durch eine geographische oder ethnische Einschränkung noch einmal die Erst- und/oder Einmaligkeit des Sieges. So ist es nicht verwunderlich, dass wir diese Inanspruchnahme eines Rekordes so zahlreich, geradezu inflationär oft vorfinden.

Eine andere Möglichkeit, Rekorde aufzustellen, strebte man durch spezielles Training und bewusste Auswahl der Disziplinen an. Nach gründlichem Studium der bestehenden Rekorde, wobei man feststellen musste, dass einige nicht zu überbieten waren, suchte man gezielt nach Kombinationssiegen, die in dieser Form noch nicht existierten.

Eine weitere Variante, Rekorde für sich in Anspruch zu nehmen, machten römische Wagenlenker durch konstruierte Vergleiche. Die Zahl der Siege und die Höhe der Preisgelder, sowie Art und Weise der Siege, als auch Erfolge mit bestimmten Rennpferden und Triumphe bei besonderen Veranstaltungen 
waren Gegenstand des Vergleichs. Teils verglich man seine Leistungen mit denen einzelner Kollegen, teils mit den zusammengefassten Erfolgen mehrer Berühmtheiten. Es erweckte schon den Eindruck von Rekordmanie, wenn man sehen musste, wie die teilweise kuriosen und grotesken Vergleiche so gedreht wurden, bis es eine Bestmarke darstellte und man somit als Rekordmann sich rühmen konnte.

Die Auswertung der Sportarten und -disziplinen, die nach unserem Verständnis den Kanon des antiken Sports bilden, erbrachten die Herausstellung von sieben Wesenszügen, die den antiken Sport charakterisieren. Sie sind nicht nur für ihre Epoche hinsichtlich Sport und Gesellschaft prägende Wesenszüge, sondern zeigen auch auffällig Gemeinsamkeiten zum Sport anderer Kulturkreise und Epochen auf.

Einige Charakteristika wirken geradezu deckungsgleich mit Wesenszügen, die uns aus unserem täglichen Sportbetrieb geläufig sind (großes öffentliches Interesse, hohe Preisgelder, Starkult u. v. m. bis zu übersteigertem Rekordstreben 
5.

5.1

AAW = E. N. GARDINER, Athletics of the Ancient World, Oxford 1930 (ND Chicago 1979)

Anth. Pal. = Anthologia Palatina

CAF $\quad=$ Citius Altius Fortius

ClG $=$ Corpus I nscriptionum Graecarum

$\mathrm{Cl}=$ Corpus Inscriptionum Latinarum

C HSPE $=$ Canadian J ournal of History of Sport and Physical Education

DNP $\quad=$ Der Neue Pauly

D-S $=$ Ch. DAREMBERG/E. SAGLIO, Dictionnaire des Antiquités Grecques et Romaines

DTZ = Deutsche Turn-Zeitung

GAA $=$ H. A. HARRIS, Greek Athletes and Athletics, London 1964

GASF = E. N. GARDINER, Greek Athletic Sports and Festivals, Dubuque 1902

GIBM = Ancient Greek Inscriptions in the British Museum

$\mathrm{GL}=\mathrm{H}$. ÜBERHORST (Hrsg.), Geschichte der Leibesübungen, Berlin/München/Frankfurt 1972-1988

$\mathrm{HfL}=$ Hochschulblatt für Leibesübungen

IAG = L. MORETTI, Iscrizioni agonistiche greche, Rom 1953

IG = Inscriptiones Graecae

IGR = Inscriptiones Graecae ad res Romanas pertinentes

IK $=$ Inschriften griechischer Städte aus Kleinasien

ILS $=\mathrm{H}$. DESSAU, Inscriptiones Latinae selectae, 3 Bde., Berlin $1923 \mathrm{ff}$.

IVM = O. KERN, Die Inschriften von Magnesia am Maeander, 
Berlin 1900

IVO = W. DITTENBERGER/K. PURGOLD, Die Inschriften von Olympia, Berlin 1896

$\mathrm{JSH}=$ J ournal of Sport History

KBSW $=$ KöIner Beiträge zur Sportwissenschaft

LkE $\quad=\quad$ Leibesübungen und körperliche Erziehung

MAMA $=$ Monumenta Asiae Minoris Antiqua

OF $\quad=$ Olympisches Feuer

OMS = ROBERT, L., Opera minora selecta: épigraphie et antiquités grècques, 7 Bde., Amsterdam 1969-1990

POxy = B. P. GRENFELL/A. S. HUNT (Hrsg.), The Oxyrhynchus Papyri

QGAA = WEILER, I. (Hrsg.) Quellendokumentation zur Gymnastik und Agonistik im Altertum, 7 Bde., Wien/Köln/Weimar 1991-2002

RE $\quad=$ Paulys Real-Encyklopädie der classischen Altertumswissenschaft, Stuttgart 1893-1980

SEG $=$ Supplementum Epigraphicum Graecum

SG $=$ L. FRIEDLÄNDER, Darstellungen aus der Sittengeschichte Roms, Bd. 1-4, Leipzig $1922^{10}$

SGR $=$ H. A. HARRIS, Sport in Greece and Rome, Ithaca 1972

Syll. $^{3}=$ W. DITTENBERGER, Sylloge inscriptionum Graecarum ${ }^{3}$, Leipzig 1915-1924 (ND 1960)

TAM $=$ Tituli Asiae Minoris 
AlGNER, $\mathrm{H}$.

AlGNER, $\mathrm{H}$.

AJ OOTIAN, A.

ALBANIDIS, E. u. a.

ALBERS, V. von

ALBRECHT, M. V.I

SCHUBERT, W. (Hrsg.)

ALFÖLDI, A.

ALTROCK, $\mathrm{H}$.

ALTROCK, $\mathrm{H}$.

AMELOTTI, M.

ANASTASIOU, A.

ANDERSON, J. G. C

ANDERSON, J. G. C.
Zur gesellschaftlichen Stellung von Henkern, Gladiatoren und Berufsathleten, in: I. WEILER (Hrsg.), Randgruppen , 200-220 Zur Organisation des Berufsathletentums in der Antike, in: Civitas 40.11, 1985, 295-298

Heroic and Athletic Sortition at Ancient Olympia, in: G. P. SCHAUS/S. R. WENN (Hrsg.), Onward, 115-129 The Joint Participation of Greeks and Barbarians in Athletic Activities during the Hellenistic and Roman Times, in: Nikephoros 19, 2006, 187-226 Das süße Brot der Helden. Wettkämpfe als Teil der Unterhaltungsindustrie, in:

E. KÖHNE/C. EWIGLEBEN (Hrsg.), Caesaren,149 159

Musik in Antike und Neuzeit, Frankfurt 1987

Die Kontorniat-Medaillons, 2 Bde., Berlin 1976

Die geistigen Bindungen des Sports in der abendländischen Kultur, Stud Gen 13, 1960, 21-28

Die kulturellen Aufgaben des Deutschen Sports, Kevelaer 1949

La posizione degli atleti di fronte al diritto romano, in: SDHI 21, 1955, 123-156

On the Original Meaning of the Gladiatorial Games, in: Nikephoros 23, 2010, 7-17

Festivals of Mên Askaênos in the Roman Colonia at Antioch of Pisida, in: JRS 3, 1913, 267-300

A Summer in Phrygia II, in: J HS 18, 1898, 81-128 
ANDRÉ, J.-M.

ANDRÉ, J.-M.
Griechische Feste, römische Spiele. Die Freizeitkultur der Antike, Stuttgart 1994

Die Zuschauerschaft als sozialpolitischer Mikrokosmos zur Zeit des Hochprinzipats, in:

J. BLÄNSDORF (Hrsg.), Theater, 165-173

ANGELI BERNARDINI, P. Esaltazione e critica dell'atletismo nella poesia greca dal VII al V sec. a. C. Storia di un'ídeologia, in: Stadion 6, 1980, 81-111

ANGELONE, R. L'agenzia di un lanista in Pompei allinsegna di un famoso combattimento gladiatorio, in: AAP 38, 1989, 339-359

ANGELOV, A./

CONRAD, S./

LUPPE, W.

ARAPOGIANNE, $X$.

ARIETI, J. A.

ARRIGONI, G.

ARRIGONI, G. (Hrsg.) Le donne in Grecia, Rom 1985

AUGUET, R.

AURI GEMMA, S.

AYDAŞ, $M$.

BACKHAUS, W.

BALABANĒS, P. D.

BALIL, A.
Cruauté et civilisation. Les jeux Romains, Paris 1970

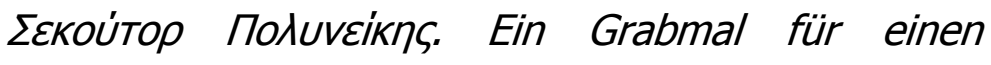
erfolgreichen Gladiator aus Marcianopolis, in: Nikephoros 9, 1996, 135-144

Olympia: The Cradle of the Olympic Games, Athen 2004

Nudity in Greek Athletics, in: CW 68, 1975, 431-436

Donne e sport nel mondo greco: Religione e società, in: G. ARRIGONI, (HRSG.) Donne, 55-201 I mosaici di Zliten, Rom/Mailand 1926

Sechs Grabstelen für Gladiatoren, in: EA 39, 2006, 105-110

Öffentliche Spiele, Sport und Gesellschaft in der römischen Antike, in: GL, Bd. 2, 200-249

Games and Sanctuaries in Ancient Greece: Olympia, Delphi, Isthmia, Nemea, Athens, Los Angeles 2004 La ley gladiatora de Italica, Madrid 1961 
BALIL, A.

BALSDON, J. P. V. D. Life and Leisure in Ancient Rome, London $1976^{2}$

BALTRUSCH, E.

BALTRUSCH, E.

BALTRUSCH, E.

BARTON, C. A.

BEAN, G. E.

BEARD, $M$.

BECK, $\mathrm{H}$.

BECK, $\mathrm{H}$.

BECK, H. (Hrsg.)

BEHRINGER, W.

BEHRWALD, $R$.

BELL, S. (Hrsg.)
Sugli spettacoli di anfiteatro, in: RAYMOND CHEVALIER (Hrsg.), Mélanges d'archéologie et d'histoire offerts à André Piganiol, Paris 1966, 357368

Politik, Kommerz, Doping: Zum Sport in der Antike, in: Gymnasium 104, 1997, 509-521

Regimen morum (=Vestigia, Beiträge zur Alten Geschichte, Bd. 41), München 1988

Die Verstaatlichung der Gladiatorenspiele, in: Hermes 116, 1988, 324-337

The Sorrows of the Ancient Romans. The Gladiator and the Monster, Princeton/New J ersey 1992 Victory in the Pentathlon, in: AJ A 60, 1956, 361-368 Pompeji: The Life of a Roman Town, London 2008 Ephebie - Ritual - Geschichte. Polisfest und historische Erinnerung im klassischen Griechenland, in: H. BECK (Hrsg.), Feiern, 55-82

Feiern und Erinnern - eine Einleitung, in: H. BECK (Hrsg.), Feiern, 9-54

Feiern und Erinnern: Geschichtsbilder im Spiegel antiker Feste, Berlin 2009

Kulturgeschichte des Sports vom antiken Olympia bis ins 21. Jahrhundert, München 2012

Festkalender der frühen Kaiserzeit als Medien der Erinnerung, in: H. BECK (Hrsg.), Feiern, 141-166 Games and Festivals in Classical Antiquity: Proceedings of the Conference Held in Edinburgh 1012 J uly 2000, Oxford 2004 
BELLEN, H./

HEI NEN, H. (Hrsg.)

BENGSTON, $\mathrm{H}$.

BENGTSON, $\mathrm{H}$.

BENTZ, M.

BERGER, E.

BERGER-HAAS, L.

BERGMANN, M.

BERGMANN, B./

KONDOLEON,

(Hrsg.)

BERLAN-BAJ ARD, A. BERNAND, A.

BERNETT, $\mathrm{H}$.
Fünfzig Jahre Forschungen zur antiken Sklaverei an der Mainzer Akademie 1950-2000. Miscellanea zum Jubiläum (=Forschungen zur antiken Sklaverei 35), Stuttgart 2001

Die Olympischen Spiele in der Antike, München $1983^{3}$ Aus der Lebensgeschichte eines griechischen Distanzläufers, in: SO 32, 1956, 35-39

Panathenäische Preisamphoren. Eine athenische Vasengattung und ihre Funktion vom 6.-4. Jahrhundert v. Chr., (18. Beih. Antike Kunst), Basel 1998

Das Basler Arztrelief: Studien zum Griechischen Grabund Votivrelief um $500 \mathrm{v}$. Chr. und zur vorhippokratischen Medizin (Robert Käppeli zum 70. Geburtstag am 21. J uli 1970), Basel 1970 Gladiatores tunicati, in: M. ROHDE-LIEGLE (Hrsg.), Gestalt und Geschichte, Festschrift für Karl Schefold, Bern 1967, 36-83

Der Koloss Neros, die Domus Aurea und der Mentalitätswandel im Rom der frühen Kaiserzeit, Mainz 1994

The Art of Ancient Spectacle (=Studies in the History C. of Art 56), New Haven/London 1999

Les spectacles aquatiques romains, Rom 2006 The Road to Olympia: Origins of the Olympic Games, London 2003

Die pädagogische Neugestaltung der bürgerlichen Leibesübungen durch die Philanthropen, (=Beiträge zur Lehre und Forschung der Leibeserziehung, Bd. 6), Schorndorf $1971^{3}$ 
BERNSTEIN, F.

BERVE, $\mathrm{H}$.

BESTE, H.-J.

BEITINALI-

GRAEBER, D.

BIANCO, G.

BIERS, W. R./

GEAGAN, D. J .

BILIŃSKI, $B$.

BILIŃSKI, B.

BILIŃSKI, B.

BINSFELD, W.

BLÄNSDORF, J. (Hrsg.)

BJ 160, 1960, 161-168

Un antico cavallo di razza nella storia delle gare circensi, in: RIL 111, 1977, 313-333

A New List of the Victors in the Caesarea at Isthmia, in: Hesperia 39, 1970, 79-93

Agoni ginnici. Componenti artistiche ed intellettuali nell'antica agonistica greca, Rom 1979

L'agonistica sportiva nella Grecia antica. Aspetti sociale e ispirazioni letterarie, Rom 1961

L'hemérodrome Philonidès, son record et la nouvelle inscription d'Aigion, in: Eos 50, 1959-60, 69-80

Zwei neue Inschriften zum Kölner Amphitheater, in:

Theater und Gesellschaft im Imperium Romanum (=Mainzer Forschungen zu Drama und Theater 4), Tübingen 1990

BLAKE, M. E.

Mosaics of the Late Empire in Rome and Vicinity, in: MAAR 17, 1940, 108-130

BLAKE, M. E.
Roman Mosaics of the Second Century in Italy, in:

MAAR 13, 1936, 67-214 
BLÁZQUEZ, J. M.

BLECH, M.

BLÜMEL, C.

BLÜMEL, C.

BOEGEHOLD, A. L.

BÖHM, J.

BOEHRI NGER, F.

BOESLAGER, D.

BÖHME, G.

BOHNE, A.

BOLLINGER, T.

BOMGARDNER, D. L.

BOMGARDNER, D. L.

BOMGARDNER, D. L.
Representaciones de gladiadores en el Museo Arqueológico Nacional, in: Zephyrus 9, 1958, 79-94 Studien zum Kranz bei den Griechen, (=RW, Bd. 38), Berlin/New York 1982 Sport der Hellenen, Berlin 1936 Sport und Spiel bei Griechen und Römern, Berlin 1934

Group and Single Competitions at the Panathenaia, in: J. NEI LS (Hrsg.), Worshipping Athena, 95-105 Die Leibesübungen im Dionysosepos des Nonnos von Panopolis, Diss. Wien 1947

Cultes d'athlètes en Grèce classiques: Propos politiques, discours mythiques, in: REA 81, 1979, 518

Das Gladiatorenmosaik in Köln, in: KJ 20, 1987, 111128

Zeit und Zahl, Frankfurt 1974

Bilder vom Sport: Untersuchungen zur Ikonographie römischer Athleten-Darstellungen, (=Nikephoros Beihefte 19), Hildesheim 2010

Theatralis licentia. Die Publikumsdemonstrationen an den öffentlichen Spiele im Rom der früheren Kaiserzeit und ihre Bedeutung im politischen Leben, Winterthur 1969

Amphitheatres on the Fringe, in: JRA 4, 1991, 282294

A New Era for Amphitheatre Studies, in: JRA 6, 1993, 375-390

The Story of the Roman Amphitheatre, London/New York 2000 
BONFANTE, L.

BORSARI, L.

BOULEY, E.

BOUSQUET, J .

BOWES, K. u. a.

BOWRA, C. M.

BRADLEY, K. R.

BRANDT, A.

BRANTLINGER, R.

BREIN, F.

BRIERS, A.

BRÖDNER, E.

BROPHY, R. H.

BROPHY, R. H./

BROPHY, M.

BROWN, Sh.

BROWN, Sh.
Nudity as a Costume in Classical Art, in: AlA 93, 1989, 543-570

L'epigrafe onorario di Avilius Teres agitatore circense, in: BCAR 30, 1902,177-185

Jeux romains dans les provinces balkanodanubiennes du // siècle après J. - C., Besançon 2001 Inscriptions de Delphes, in: BCH 116, 1992, 177-196

An Amphitheatre and its Afterlives, in: JRA 16, 2003, 380-394

Pindar, Oxford 1964

The Chronology of Nero's Visit to Greece $A D$ 66/67, in: Latomus 37, 1978, 61-72

Moralische Werte in den Res gestae des Ammianus Marcellinus (=Hypomnemata 122), Göttingen 1999 Bread and Circuses. Theories of Mass Culture as Social Decay, Ithaka, NY, 1983

Die Leibesübungen im alten Griechenland, in: GL 2, 82-167

Sporting Success in Ancient Greece and Rome, Oxford 1994

Die römischen Thermen und das antike Badewesen, Darmstadt 1983

Deaths in the Panhellenic Games. Arrichion and Creugas, in: AJPh 99, 1978, 363-390

Deaths in the Panhellenic Games, I/: All combative sports, in: AJPh 106, 1985, 171-198

Death and Decoration: Scenes from the Arena on Roman Domestic Mosaics, in: RICHLIN, A. (Hrsg.), Pornography, 180-211

Explaining the Arena: Did the Romans "need" Gladiators?, in: J RA 8, 1995, 376-384 
BROWNE, R. B.

BUCKLER, H. W.

BUHMANN, $\mathrm{H}$.

BUHMANN, $\mathrm{H}$.

BULANDA, E.

BUONOCORE, M.

BURNETT, A. P.

CALDELLI, M. L.

CALDELLI, M. L.

CALDELLI, M. L.

CAMERON, A.

CAMERON, A.

CAMERON, A.

CAMERON, A.

CANALI DE ROSSI, F.

CARTER, J. M.
Rituals and Ceremonies in Popular Culture, Ohio 1980 Lydian Records, in: JHS 37, 1917, 88-115

Die Olympischen Spiele im Altertum, in: Anregung 30, 1984, 406-414

Der Sieg in Olympia und in den anderen panhellenischen Spielen, München $1975^{2}$

Bogen und Pfeil bei den Völkern des Altertums, Wien/Leipzig 1913

Epigrafia anfiteatrale dell'occidente romano, I/l. Regiones Italiae I/-V, Sicilia, Sardino et Corsica (=Vetera 6), Rom 1992

Odes for Victorious Athlets, Baltimore 2010

L'agon Capitolinus. Storia e protagonisti dall'istituzione domizianea al IV secolo, Rom 1993

Gli agoni alla greca nelle regioni occidentali dell'impero: La Gallia Narbonensis, Rom 1997

Curia athletarum, hiera xystike synodos $e$ organizzazione delle terme a Roma, in: ZPE 93, 1992, 75-87

Circus Factions. Blues and Greens at Rome and Byzantium, Oxford 1976

Consular Diptychs in Their Social Context. New Eastern Evidence, in: JRA 11, 1998, 385-403

Porphyrius the Charioteer, Oxford 1973

Das späte Rom 284-430 n. Chr., München 1994

Hippiká/Corse di cavalli e di carri in Grecia, Etruria e Roma, I. - La gara delle quadrighe nel mondo greco, (=Nikephoros Beihefte 18), Hildesheim 2011

Sports and Pastimes of the Middle Ages, Lauham/New York/London 1988 
CARTER, M.

CARTER, M.

CARTER, M.

CARTER, M.

CARTER, $M$.

CARTER, J. M./

KRÜGER, A. (Hrsg.)

CARY, M./

HAARHOFF, T. J .

CAVALLARO, A. M.

CAZES, D. (Hrsg.)

CERUTTI, S.

CHAMBERLAND, G.

CHAMBERLAND, G.

CHAMBERS, $R$.

CHAMPLIN, E.
Archiereis and Asiarchs: A Gladiatorial Perspective, in: GRBS 44, 2004, 41-68

A Doctor Secutorum and the Retiarius Draukos from Corinth, in: ZPE 126, 1999, 262-268

Gladiatorial Ranking and the "SC de Pretiis Gladiatorum Minuendis" (CIL II 6278 = ILS 5163), in: Phoenix 57, 2003, 83-114

Gladiators and Monomachoi: Greek Attitudes to a Roman ,Cultural Performance', in:

Z. PAPAKONSTANTINOU (Hrsg.), Cultures, 150-174 The Roman Spectacles of Antiochus IV Epiphanes at Daphne 166 B. C., in: Nikephoros 14, 2001, 45-62 Ritual and Record. Sports Records and Quantification in Pre-Modern Societies, New York, Westport (Connecticut), London 1990

Life and Thought in the Greek and Roman World, London $1946^{4}$

Spese e spettacoli. Aspetti economici - strutturali degli spettacoli nelle Roma giulio-claudia, (=Antiquitas 1. Abhandlungen zur Alten Geschichte 34), Bonn 1984

Le cirque romain, Toulouse 1990

The Seven Eggs of the Circus Maximus, in: Nikephoros 6, 1993, 167-176

The Organisation of Gladiatorial Games in Italy, in: JRA 12, 1999, 613-616

Remarques sur quatre inscriptions „amphithéâtrales" italiennes, in: ZPE 156, 2006, 284-292

Greek Athletics and the Jews, Oxford, Ohio 1980 Nero, Cambridge, MA 2004 
CHARLESWORTH, M. P. Die Tugenden eines römischen Herrschers, in: H. KLOFT (Hrsg.), Ideologie und Herrschaft in der Antike, Darmstadt 1979, 361-387

CHESKA, A. The Anthropology of Sport: An Introduction, S. Hadley, Mass. 1985

CHEVALIER, R. (Hrsg.) Mélanges d'archéologie et d'histoire offerts à André Piganiol

CHRISTESEN, P.

Whence 776? The Origin of the Date for the First Olympiad, in: Z. PAPAKONSTANTINOU (Hrsg.), Cultures, 13-34

CHRISTOPOULOS, L. Early Combat Sports Rituals in China and the Rise of Professionalism (475 BC-220 AD), in: Nikephoros 23, 2010, 19-41

CHRONĒS, M. Olympiakoi Agōnes, Athen 2002

CLAUSS, $M$.

Kaiser und Gott: Herrscherkult im Römischen Reich, Stuttgart 1999

CLAVEL-LÉVÊQUE, M. L'espace des jeux dans le monde romaine: hégémonie, symbolique et practique sociale, in: ANRW II 16.3 (1986), 2405-2563

COARELLI, F. // rilievo con scene gladiatorie, in: Stud Misc 10, 1967, 85-99

COLEMAN, K. M. Fatal Charades: Roman Executions Stages as Mythological Enactements, in: JRS 80, 1990, 44-73

COLEMAN, K. M. Launching into History: Aquatic Displays in the Early Empire, in: JRS 83, 1993, 48-74

COLEMAN, K. M. Ptolemy Philadelphus and the Roman Amphitheater, in: W. J. SLATER, (Hrsg.), Roman Theater, 49-68

COLIN, J. Affiches et invitations pour les spectacles de gladiateurs, in: Pallas 4, 1956, 51-57

COLINI, A./COZZA, L. L Ludus Magnus, Rom 1962

CONFORTO, M. L. u. a. Anfiteatro Flavio, Rom 1988 
CONNOLLY, P.

CORBEILL, A.

CROSBY, A.

CROWTHER, N. B.

CROWTHER, N. B.

CROWTHER, N. B.

CROWTHER, N. B.

CROWTHER, N. B.

CROWTHER, N. B.

CROWTHER, N. B.

CROWTHER, N. B.

CROWTHER, N. B.

CROWTHER, N. B.
Colosseum. Arena der Gladiatoren, Mainz 2005

Thumbs in Ancient Rome: Pollex as Index, in: MAAR $42,1997,1-21$

The Measure of Reality: Quantification and Western Society 1250-1600, Cambridge 1997

"Sed quis custodiet ipsos custodes?" The Impartiality of the Olympic Judges and the Case of Leon of Ambracia, in: Nikephoros 10, 1997, 149-160 (= Athletika 71-81)

The Age-Category of Boys at Olympia, in: Phoenix 42.4, 1988, 304-308 (= Athletika 76-92)

The Ancient Olympics and Their Ideals, in: G. P. SCHAUS/S. R. WENN (Hrsg.), Onward, 2007, $69-80$

Athlete and State: Qualifying for the Olympic Games in Ancient Greece, in: JSH 23.1, 1996, 34-43 (= Athletika 23-33)

Athletic Dress and Nudity in Greek Athletics, in. Eranos 80, 1982, 163-168 (=Athletika 135-140)

Athletika. Studies on the Olympic Games and Greek Athletics, in: DECKER, W./WEILER, I. (Hrsg.), Nikephoros Beihefte Bd. 11, Hildesheim 2004 Boy Victors at Olympia, in: AC 58, 1989, 206-210 (=Athletika 109-113)

The Evidence for Kicking in Greek Boxing, in: AJ Ph 111, 1990, 176-181 (= Athletika 223-227)

The Finish in the Greek Foot-Race, in: Nikephoros 12, 1999, 131-142 (= Athletika 203-213)

Flogging as a Punishment in the Ancient Games, in: Nikephoros 11,1998, 51-82 (= Athletika 141-168) 
CROWTHER, N. B.

CROWTHER, N. B.

CROWTHER, N. B.

CROWTHER, N. B.

CROWTHER, N. B.

CROWTHER, N. B.

CROWTHER, N. B.

CROWTHER, N. B.

CROWTHER, N. B.

CROWTHER, N. B.

CROWTHER, N. B.

CROWTHER, N. B.
Greek Equestrian Events in the Late Republic and Early Empire: Africanus and the Olympic Victory Lists, in: Nikephoros 8, 1995, 111-123 (=Athletika 121-131)

Greek Games in Republican Rome, in: AC 52, 1983, 268-273 (=Athletika 381-385)

Nudity and Morality: Athletics in Ancient Italy, in: C] 76.2, 1980-1981, 119-123 (=Athletika 375-379)

Numbers of Contestants in Greek Athletic Contest, in: Nikephoros 6, 1993, 39-52 (=Athletika171-182) Observations on Boys, Girls, Youths and Age Categories in Roman Sports and Spectacles, in: Z. PAPAKONSTANTINOU (Hrsg.), Cultures, 195-216 The Olympic Training Period, in: Nikephoros 4, 1991, 161-166 (= Athletika 65-70)

Reflections on Greek Equestrian Events: Violence and Spectator Attitudes, in: Nikephoros 7, 1994, 121-133 (=Athletika 229-240)

The Role of Heralds and Trumpeters at Greek Athletic Festivals, in: Nikephoros 7, 1994, 135-155 (=Athletika 183-201)

Rounds and Byes in Greek Athletics, in: Stadion 18.1, 1992, 68-74 (= Athletika 215-220)

The Sebastian Games at Naples (IvO 56), in: ZPE 79, 1989, 100-102 (=Athletika 93-96)

Victories without Competition in the Greek Games, in: Nikephoros 14, 2001, 29-44 (= Athletika 281295)

Visiting the Olympic Games in Ancient Greece: Travel and Conditions for Athletes and Spectators, in: IJHS 18.4., 2001, 37-52 (= Athletika 35-50) 
CROWTHER, N. B. Weightlifting in Antiquity: Achievement and Training, in: G \& R 24, 1977, 111-120 (=Athletika 269-277)

CROWTHER, N. B. The Ancient Olympics and Their Ideals, in: G. P. SCHAUS/S. R. WENN (Hrsg.), Onward, 69-80

CROWTHER, N. B. Slaves and Greek Athletics, in: QUCC 40.1, 1992, 3542 (=Athletica 247-253)

CURRIE, B. Pindar and the Cult of Heroes, Oxford 2005

DALBY, A.

DECKER, W. Empire of Pleasures. Luxury and Indulgence in the Roman World, London 2000

DECKER, W.

Bibliographie zum Sport im Altertum, Köln 2005

Der Rekord des Rituals. Zum sportlichen Rekord im Alten Ägypten, in: G. SPITZER/D. SCHMI DT (Hrsg.), Eigenständigkeit, 66-74

(= The Record of the Ritual: The Athletic Records of Ancient Egypt, in: J. M. CARTER/A. KRÜGER (Hrsg.), Ritual, 21-30)

DECKER, W. In: DNP 11, s. v. Sport, 2001, 838-846

DECKER, W. Sport in der griechischen Antike: vom minoischen Wettkampf bis zu den Olympischen Spielen, Hildesheim 2012

DECKER, W. In: DNP 11, s. v. Sportfeste, 2001, 847-855

DECKER, W. u. a. Jahresbibliographie zum Sport im Altertum, in: Nikephoros 2 ff., 1989 ff.

DECKER, W. Neue Olympiasieger aus Ägypten, in: W. WAITKUS(Hrsg.), Diener des Horus, Aegyptiaca Hamburgensia Bd. 1, Gladbeck 2008, 67, n. 2 
DECKER, W.

Olympiasieger

aus

Ägypten,

in:

E. GRAEFE/U. VERHOEVEN (Hrsg.), Religion und Philosophie im Alten Ägypten. Festgabe für PHILIPPE DERCHAIN zu seinem 65. Geburtstag am 24. Juli 1991, Leuven 1991, 93-105

DECKER, W.

Zur Vorbereitung und Organisation griechischer Agone, in: Nikephoros 10, 1997, 77-102

DECKER, W./

Le sport dans l'antiquité. Égypte, Grèce, Rome,

THUI LLIER, J.-P.

Paris 2004

DEUBNER, L.

Kult und Spiel im alten Olympia, Leipzig 1936

DIEBNER, S.

Asernia-Venafrum. Untersuchungen zu den römischen Steindenkmälern zweier Landstädte Mittelitaliens, Rom 1979

DIEM, C.

DIEM, C. Weltgeschichte des Sports und der Leibeserziehung, Stuttgart $1971^{3}$

Wesen und Lehre des Sports und der Leibeserziehung, Berlin u.a. $1964^{2}$

DODGE, $\mathrm{H}$.

Amusing the Masses: Buildings for Entertainment and Leisure in the Roman World, in:

POTTER, D./MATTINGLY, D. (Hrsg.), Life, 205-255

DOLCH, M. Wettkampf, Wasserrevue oder diätetische Übungen? Das Mosaik mit den zehn Mädchen in der römischen Villa bei Piazza Armerina auf Sizilien, in: Nikephoros 6.5, 1992, 173-189

DOMERGUE, E. C./ Spectacula I. Gladiateurs et Amphithéâtres, Lattes LANDES, Ch./ 1990

PAI LLIER, J.-M. (Hrsg.)

DÖRFELD, W.

Metrologische Beiträge, V. Das äginäisch-attische Maßsystem, in: MDAI (A) 15, 1890, 167-187

DREES, L. Olympia. Götter, Künstler und Athleten, Schorndorf 1967 
DREES, L.

DREXEL, F.

DUNBABIN, K. M. D.

DUNNING, E. (Hrsg.)

DUNST, G.

DUNST, G.

DURAND, M.

EBERT, J .

EBERT, J.

EBERT, J.

EBERT, J .

EBERT, J .

EBERT, J .
Der Ursprung der Olympischen Spiele, Schorndorf 1962

Drei Inschriften von Wagenlenkern, in:

L. FRIEDLÄNDER, SG Bd. 4, 179-196

The Mosaics of Roman North Africa. Studies in Iconography and Patronage, Oxford 1978

Sociology of Sport: A Selection of Readings, London 1971

Die Inschrift des Periodoniken Leon, in: ZPE 3, 1968, 139-148

Die Siegerliste der samischen Heraia, in: ZPE 1, 1967, 225-239

La compétition en Grèce antique. Généalogie, évolution, interprétation, Paris 1999

Agonismata.Kleine philologische Schriften zur Literatur, Geschichte und Kultur der Antike Stuttgart 1997

Zum Epigramm auf den Schwerathleten Aurelios Achilleus aus Aphrodisias, in: Stadion 7, 1981, 203210

Zu Fackelläufen und anderen Problemen in einer griechischen agonistischen Inschrift aus Ägypten, in: Stadion 5, 1979, 1-19

Griechische Epigramme auf Sieger an gymnischen und hippischen Agonen, Berlin 1972

Zu griechischen agonistischen Inschriften, in: WZ Halle 15, 1966, 375-387

Zur neuen Bronzeplatte mit Siegerinschriften aus Olympia (Inv. 1148), in: Nikephoros 10, 1997, 217233 
EBERT, J .

EBERT, J .

EBERT, J.

EBERT, J .

EBNER, M./ESCH-

WERMELING (Hrsg.)

EDMONDSON, J. C.
Neues zu den Inschriften für Aurelios Heras aus Chios, in: Nikephoros 1, 1988, 85-102

Neues zum Hippodrom und zu den hippischen Konkurrenzen in Olympia, in: Nikephoros 2, 1989, 89-108

Noch einmal zum Sieg im Pentathlon, in: ZPE 13,1974, 257-262

Zum Pentathlon der Antike, Berlin 1963

Kaiserkult, Wirtschaft und Spectacula: Zum politischen und gesellschaftlichen Umfeld der Offenbarung, Göttingen 2010

Dynamic Arenas: Gladiatorical Presentations in the City of Rome and the Construction of Roman Society during the Early Empire, in: W. J. SLATER (Hrsg.), Roman Theater, 69-112

EDWARDS, C. The Politics of Immorality in Ancient Rome, Cambridge 1993

EGELHAAF-GAISER, U./ Spektakuläre Spiele im Amphitheater, Göttingen FUCHS, TH. (Hrsg.) 2006

EICHBERG, $\mathrm{H}$.

Der Beginn des modernen Leistens, in: Sportwissenschaft 4, 1974, 21-48

EICHBERG, $\mathrm{H}$.

EICHBERG, $\mathrm{H}$. Zur historisch-kulturellen Relativität des Leistens in Spiel und Sport, in: Sportwissenschaft 6, 1976, 9-34 Leistung, Spannung, Geschwindigkeit. Sport und Tanz im gesellschaftlichen Wandel des 18./19. Jahrhunderts (=Stuttgarter Beiträge zur Geschichte und Politik 12), Stuttgart 1978

EICHBERG, $\mathrm{H}$.
Recording and Quantifying Performance is Not Natural. A Reply to Krüger and Ito, in: Stadion 3, 1977, 253-256 
EICHBERG, $\mathrm{H}$.

EICHBERG, $\mathrm{H}$.

EICHBERG, $\mathrm{H}$.

ELIAS, N.
Die Veränderung des Sports ist gesellschaftlich, Münster 1986

Der Weg des Sports in die industrielle Zivilisation, Baden-Baden 1973

Auf Zoll und Quintlein. Sport und

Quantifizierungsprozeß in der frühen Neuzeit, in:

Archiv für Kulturgeschichte 56, 1974, 141-176

Über den Prozeß der Zivilisation. Wandlungen der Gesellschaft: Entwurf zu einer Theorie der Zivilisation, Bd. 2, Frankfurt a. M. 2009

ELIAS, N./DUNNING, E. Sport im Zivilisationsprozeß. Studien zur Figurationssoziologie, Münster $1984^{2}$

EWIGLEBEN, C. „Der blanke Stahl ist's den sie lieben“: die Akteure und ihr Publikum, in: E. KÖHNE/C. EWIGLEBEN (Hrsg.), Caesaren, 131-148

FACCENA, D. Rilievi Gladiatori, in: BCAR 76, 1959, 37-75

FAGAN, G. Bathing in Public in the Roman World, Ann Arbor 1999

FARRINGTON, A.

Olympic Victors and the Popularity of the Olympic Games in the Imperial Period, in: Tyche 12, 1997, 15-46

FARRINGTON, A.

Isthmionikai. A Catalogue of Isthmian Victors, (=Nikephoros Beihefte 21), Hildesheim 2012

FEAR, A. T. Status Symbol or Leisure Pursuit? Amphitheatres in the Roman World, in: Latomus 59, 2000, 82-87

FINLEY, M. I. The Ancient Economy, London 1973

FINLEY, M. I./ Die Olympischen Spiele der Antike, Tübingen 1976 PLEKET, H. W.

FLAIG, E.
In: DNP 4, s. v. Gladiator, 1998, 1075-1078 
FLAIG, E.

Ritualisierte Politik. Zeichen, Gesten und Herrschaft im alten Rom (=Historische Semantik 1), Göttingen 2003

FLAIG, E.

Roman Gladiatorial Games. Ritual and Political Consensus, in: JRA Supplementary Series 66, 83-92

FLESCHENBERG, O. von Spätantike Anleitung zum Bogenschießen, in: WS $60,1942,43-70$

FLORIANI

Circhi e spettacoli circensi nelle province romane

SQUARCIAPINO, $M$. d'Africa, in: RAL 34, 1979, 275-290

FORBES, C. A.

Accidents and Fatalities in Greek Athletics, in: Classical Studies in Honour of W. A. Oldfather, Urbana 1943, 50-59

FORBES, C. A. Ancient Athletic Guilds, in: CPh 50, 1955, 238-252

FORBES, C. A. Crime and Punishment in Greek Athletics, in: CJ 47, 1952, 169-173 u. 202-203

FORNÉS PALLICER, Los gestos con el pulgar en los combates de M. A./ PUIG gladiatores, in: Latomus 65, 2006, 263-971

RODRÍGUEZ

ESCALONA, $M$.

FORTUIN, R. W.

Der Sport im augusteischen Rom. Philologische und sporthistorische Untersuchungen (=Palingenesia 57), Stuttgart 1996

FRANCHI, L. Rilievo con pompa funebre e rilievo con gladiatori al museo dell'Aquila, in: Stud Mis 10, 1967, 23-32, Tav. 11,12

FRASS, $M$ Gesellschaftliche Akzeptanz "sportlicher" Frauen in der Antike, in: Nikephoros 10, 1997, 119-133

FREEMAN, Ch. Egypt, Greece and Rome. Civilizations of the Ancient Mediterraneum, Oxford 1996

FRENCH, D./ Two Gladiatorial Texts from Claudiopolis in Bithynia, ÜNDEMIŞ, $M$. in: EA 13, 1989, 91-97 
FREVEL, CH./

HESBERG, H. V. (Hrsg.) der Antike, Wiesbaden 2007

FRIEDLÄNDER, L.

FRISCH, P.

FRISCH, P.

FUCHS, $\mathrm{M}$.

FURTWÄNGLER, A.

FUTRELL, A.

GAGER, J. G. (Hrsg.) Curse Tablets and Binding Spells from the Ancient World, Oxford $1999^{2}$

GARCÍA ROMERO, F. El deporte en los proverbios griegos antiguos, Hildesheim 2001 (=Nikephoros Beihefte, Bd. 7)

GARCÍA Y BELLIDO, A. El español C. Apuleius Diocles, el más famoso corredor de carros de la antigüedad, in: CAF 14, 1972, 5-17

GARCÍA Y BELLIDO, A. Lapidas funerarias de gladiadores de Hispania, in: AEA 33, 1960, 123-144

GARDINER, E. N. Athletics of the Ancient World, Oxford 1930 (ND Chicago 1978)

GARDINER, E. N. Greek Athletic Sports and Festivals, London 1910 GARDI NER, E. N.

GARDNER, P. $1903,54-70$

Boat-Races among the Greeks, in: JHS 2, 1881, 9097

GARDNER, P. 
GARDNER, P.

GARRAFFONI, R. S

GEESE, R.

GEHRKE, H.-J .

GEOMINY, W./

LEHMANN, $\mathrm{S}$.

GERBER, D. E.

GIANNELLI, G.

GIGLIOLI, C. Q.

GILBERT, R.

GLADER, E. A.

GÖLLMANN, C.

GOLDEN, M.

GOLDEN, M.

GOLDEN, M.

GOLDSTEIN, J .
A Stele Commemorating a Victory in a Boat Race, in: JHS 11, 1890, 146-150

Gladiators' Daily Lives and Epigraphy: A Social Archaeological Approach to the Roman munera During the Early Principate, in: Nikephoros 21, 2008, 223-241

Warum sprangen die alten Griechen mit Halteren?, in: Sport Zeiten 5.3, 2005, 57-66

Die Olympischen Spiele im Rahmen des antiken Sports, in: AU 28.5, 1985, 14-31

Zum Bronzebild des sitzenden Faustkämpfers im Museo Nazionale Romano, in: Stadion 15, 1989, 139165

A Commentary on Pindar Olympian Nine, Stuttgart 2002

Culti e miti della Magna Grecia, Florenz $1963^{2}$

La corsa della fiaccola ad Atene, in: RAL 31, 1922, 315-335

Die Beziehungen zwischen Princeps und stadtrömischer Plebs im frühen Prinzipat, Bochum 1976

Amateurism and Athletics, West Point N. Y. 1978

Zur Beurteilung der öffentlichen Spiele Roms bei Tacitus, Plinius d. J. und Juvenal, Münster 1942

Sport and Society in Ancient Greece, Cambridge 1998

Sport in the Ancient World from A to Z, London 2004 Inventing Ancient Culture. Historicism, Periodization and the Ancient World, London 1997

Sports Violence, New York 1983 
GRANT, M.

GREGORI, G. L.

GRENZMANN, L./

GRUBMÜLLER, K./

RÄDLE, F./

STAEHELIN, M.

GRODDE, O.

GRÖSSING, St.

GROOT, $\mathrm{H}$.

GROßSCHMIDT, K./

KANZ, F. (Hrsg.)

GRÜNDEL, L.

GUNDERSON, E.

GÜNTHER, L.-M.

GÜNTHER, R.

GUTTMANN, A.

GUTTMANN, A.
Gladiators, London 2000

Epigrafia anfiteatrale dell'occidente romano. II. Regiones Italiae VI-XI Rom (=Vetera 4), Rom 1989 Die Präsenz der Antike im Übergang vom Mittelalter zur frühen Neuzeit (=AAWG, phil.-hist. Kl. 3. F., Bd. 263), Göttingen 2004

Sport bei Quintilian (=Nikephoros Beihefte Bd. 3), Hildesheim 1997

Rivalität - Ritual - Rekord. Überlegungen zur Kulturgeschichte der menschlichen Bewegung (=Salzburger Universitätsreden: 74), Salzburg 1984 Zur Bedeutung der öffentlichen Spiele bei Tacitus, Sueton und Cassius Dio: Überlegungen zur Selbstbeschreibung der römischen Gesellschaft, Berlin u. a. 2008

Gladiatoren in Ephesos. Tod am Nachmittag. Katalog der Ausstellung im Ephesos Museum Selçuk, Wien 2002

Die Darstellung des Laufes in der griechischen Kunst, Würzburg 1934

The Ideology of the Arena, in: Cl Ant 15.1, 1996, 113-151

Olympia und seine Spiele: Kult - Konkurrenz Kommerz, Berlin 2004

Olympia: Kult und Spiele in der Antike, Darmstadt 2004

Forum: The Athletic, the Aesthetic and the Erotic, in:

JSH 29.3, 2002, 379-412

From Ritual to Record - The Nature of Modern Sports, New York 1978 
GUTTMANN, A.

GUTTMANN, A.

GUTTMANN, A.

HAAS, V.

HAHN, W.

HALLETT, C. H.

HARRIS, H. A.

HARRIS, H. A.

HARRIS, H. A.

HARRIS, H. A.

HARRIS, H. A.

HARRIS, H. A.

HARRIS, H. A.

HEIL, M.

HEMELRIJ K, J . M.

HERMANN, P.

HERRMANN, H.-V..

HERRMANN, H.-V.
Rituals, Records, Responses, in:

J. M. CARTER/A. KRÜGER (Hrsg.), Ritual, 153-160

Sports Spectators, New York 1986

Sports: The First Five Millennia, Amherst 2004

Kompositbogen und Bogenschießen als Wettkampf im Alten Orient, in: Nikephoros 2, 1989, 27-42

Olympiaden in Olympia, München 1992

The Roman Nude. Heroic Portrait Statuary 200 BC$A D$ 300, Oxford 2005

Greek Athletes and Athletics, London 1964 (ND 1984)

Greek Javlin-Throwing, in: G \& R 10, 1963, 26-36

The Method of Deciding Victory in the Pentathlon, in: $G \& R$ 19,1972, 60-64

Notes on Three Athletic Inscriptions, in: JHS 82, 1962, 19-24

An Olympic Epigramm. The Athletic Feats of Phayllos, in: G \& R 7, 1960, 3-8

Sport in Greece and Rome, London 1972

The Starting Gate for Chariots at Olympia, in: G \& R 15, 1968, 113-126

Die Jubilarfeiern der römischen Kaiser, in: H. BECK (Hrsg.), Feiern, 167-202

Het pentathlon, in: Hermeneus 37, 1966, 116-126

Eine Kaiserurkunde der Zeit Marc Aurels aus Milet, in: MDAl (I) 25, 1975, 149-166

Die Siegerstatuen von Olympia, in: Nikephoros 1, 1988, 119-183

Olympia und seine Spiele im Wandel der Zeiten, in: Gymnasium 80, 1973, 172-205 
HERZ, P.

HESBERG, H./

ZANKER, P. (Hrsg.)

HEUKE, C.

HIRN, A.

HÖFER, A. (Hrsg.)

HÖNLE, A.

HÖNLE, A.

HÖNLE, A./HENZE, A. HOLLENBACK, G. M.

HOPF, W.

HOPF, W.

HOPKINS, K.

HORNBLOWER, S.

HORNUM, M. B.

HÖRRMANN, M.

HORSMANN, G.
Kaiserfeste in der Prinzipatszeit, in: ANRW 16.2, 1978, 1135-1164

Römische Gräberstraßen. Selbstdarstellung - Status

- Standard, München 1987

Circus und Hippodrom als politischer Raum, Hildesheim 1994

Ursprung und Wesen des Sports, Berlin 1936

Olympische Spiele = Olympic Games = Jeux Olympiques, Sankt Augustin 2003

In: DNP 2, s. v. Circus, 1997, 1210-1220

Olympia in der Politik der griechischen Staatenwelt, Tübingen 1968

Römische Amphitheater und Stadien, Zürich 1981 Deaths in the Pan - Hellenic Games: the Case of Arrachion Reconsidered, in: Nikephoros 23, 2010, 95104

The Development of Public Entertainment Venues in Rome and Italy, in: K. LOMAS u. a. (Hrsg.), Bread, 46-60

Kritik der Sportsoziologie, Münster $1984^{2}$

Soziale Zeit und Körperkultur, Münster 1981

Death and Renewal, Cambridge/London 1985

Thucydides and Pindar: Historical Narrative and the World of Epinikian Poetry, Oxford u. a., 2004

Nemesis. The Roman State and the Games, Leiden 1993

Religion der Athleten, Stuttgart 1968

Die Bescholtenheit der Berufssportler im römischen Recht, in: Nikephoros 7, 1994, 207-227 
HORSMANN, G. Die Wagenlenker der römischen Kaiserzeit. Untersuchungen zu ihrer sozialen Stellung. (=Forschungen zur antiken Sklaverei 29), Stuttgart 1998

HUBER, $K$.

Theorie der gymnischen Erziehung bei den Römern, Langensalza 1934

HUMPHREY, J. H. Roman Circuses: Arenas of Chariot-Racing, London 1986

HURSCHMANN, R. In: DNP 11, s. v. Sportgeräte, 2001, 855-858

HYDE, W. W. Olympic Victor Monuments and Greek Athletic Art, Washington 1921

HYLAND, A.

Equus: The Horse in the Roman World, London 1990

ISIDORI FRASCA, R. Ludi nell'antica Roma (=Attivista Motorie 3), Bologna 1980

ITGENSHORST, T. Tota illa pompa: Der Triumph in der römischen Republik [mit CD-ROM, Triumphe 340 v. Chr. bis 19 v. Chr.], Göttingen 2005

JACOBELLI, L. Gladiatori a Pompeii.Protagonisti,luoghi,immagini Rom 2003

J OHNSON, P./LING, R./ Ancient Mosaics (=J RA Suppl. 9), London 1994 SMITH, D. J.

JONES, C. P. Gladiator Epigrams from Beroea and Stratonikeia (Caria), in: ZPE 163, 2007, 45-48

JONES, C. P. Inscription to Aurelius Achilleus, in: HSt 85, 1981, 119-129

JONES, C. P. The Pancratiasts Helix and Alexander on an Ostian Mosaic, in: JRA 11, 1998, 293-298

J ONES, M. W. Designing Amphitheatres, in: MDAl (R) 100, 1993, 392- 442, pl. 82-90

JÜTHNER, J. Über antike Turngeräthe, Wien 1896 
JÜTHNER, J .

JÜTHNER, J .

JÜTHNER, J.

JÜTHNER, J./MEHL, E.

JUNKELMANN, $M$.

J UNKELMANN, M.

JUNKELMANN, M.

JUNKELMANN, M.

JUNKELMANN, M.

JUNKELMANN, M.

JUNKELMANN, M.

KANTZIOS, I.

KANZ, F./

GROSSSCHMIDT, K.

KAYSER, F.
Die athletischen Leibesübungen der Griechen, in: F. BREIN (Hrsg.), Bd. 2, Graz/Wien/Köln 1965-1968 Philostratos über Gymnastik, Leipzig/Berlin 1909 Zu Pindar Nem. 7, 70 ff., in: WS 50, 1932, 166-170 RE Suppl. 9 (1962), s. v. Pygme, 1306-1352 Mit Ben Hur am Start. Wagenrennen im Circus Maximus, in: E. KÖHNE/C. EWIGLEBEN (Hrsg.), Caesaren, 91-108

Bewaffnung und Kampftechnik der Gladiatoren, in: K. GROßSCHMIDT/F. KANZ (Hrsg.), Gladiatoren, 2542

Familia gladiatoria. Die Helden des Amphitheaters, in: E. KÖHNE/C. EWIGLEBEN (Hrsg.), Caesaren , 3980

Griechische Athleten in Rom. Boxen, Ringen und Pankration, in: E. KÖHNE/C. EWIGLEBEN (Hrsg.), Caesaren, 81-90

Hollywoods Traum von Rom: "Gladiator" und die Tradition des Monumentalfilms, Mainz 2004 Die Reiter Roms, Teil I: Reise, Jagd, Triumph und Circusrennen, (=Kulturgeschichte der antiken Welt Band 45), Mainz 1990

Das Spiel mit dem Tod. So kämpften Roms Gladiatoren, Mainz 2000

Victory, Fame and Song in Pindar's Odes, in: IJSH 21, 2004, 67-79

Stand der anthropologischen Forschungen zum Gladiatorenfriedhof in Ephesos, in: JÖAl 74, 2005, 103-123

La gladiature en Égypte, in: REA 102, 2000, 459-478 
KEFALIDOU, E.

KEMPEN, Y.

KENNELL, N. M.

KENNELL, N. M.

KENNELL, N. M.

KIPPENBERG, H. G.

KLEE, M.

KLEE, T.

KLOEREN, M.

KLOFT, $\mathrm{H}$.

KLOFT, $\mathrm{H}$.

KLOFT, $\mathrm{H}$.

KNAB, $R$.

KNOCHE, U.
Ceremonies of Athletic Victory in Ancient Greece: An Interpretation, in: Nikephoros 12, 1999, 95-119

Krieger, Boten und Athleten. Untersuchungen zum Langlauf in der griechischen Antike (=Studien zur Sportgeschichte 1), St. Augustin 1992

,NEPSN ПEPIOLONIKH $\Sigma$ ', in: AJPh 1093, 1988, 239251

The Greek Ephebate in the Roman Period, in: Z. PAPAKONSTANTINOU (Hrsg.), Cultures, 175-194 Ephebeia: A Register of Greek Cities with Citizen Training Systems in the Hellenistic and Roman Periods, (=Nikephoros Beihefte 12), Hildesheim 2006 Die Entstehung der antiken Klassengesellschaften, Frankfurt 1977

Lebensadern des Imperiums: Straßen im Römischen Reich, Darmstadt 2010

Zur Geschichte der gymnischen Agone an griechischen Festen, Leipzig/Berlin 1918

Sport und Rekord. Kultursoziologische Untersuchungen zum England des 16. bis 18. Jahrhunderts, Leipzig 1935 (ND Münster 1985)

Liberalitas principis, Bonn (Köln) 1970

Mysterienkulte der Antike. Götter - Menschen Rituale, München 1999

Die Wirtschaft der griechisch-römischen Welt, Darmstadt 1992

Die Periodoniken, Gießen 1934 (ND Chicago 1980)

Der römische Ruhmesgedanke, in: Philologus 89, 1934, 102-124 (= Römische Wertbegriffe, in: WdF 34, 1967, 420-445) 
$\mathrm{KOCH}, \mathrm{A}$.

KOCKEL, V.

KÖHNE, E.

KÖHNE, E./

EWIGLEBEN, C. (Hrsg.) KÖNEN, L.

Die Leibesübungen im Urteil der antiken und frühchristlichen Anthropologie, (=Beiträge zur Lehre und Forschung der Leibeserziehung, Bd. 20), Schorndorf 1965

Die Grabbauten vor dem Herkulaner Tor in Pompeji, (=Beiträge zur Erschließung hellenistischer und kaiserzeitlicher Skulptur und Architektur, Bd. 1), Göttingen 1983

Brot und Spiele. Die Politik der Unterhaltung, in: E. KÖHNE/C. EWIGLEBEN (Hrsg.), Caesaren 13-38 Caesaren und Gladiatoren [Begleitbuch zur Ausstellung], Mainz 2000

Eine agonistische Inschrift aus Ägypten und frühptolemäische Königsfeste, Meisenheim 1977 (=Beiträge zur klassischen Philologie, Heft 56)

KÖNIG, A./KÖNIG, I. Der römische Festkalender der Republik: Feste, Organisationen und Priesterschaften, Stuttgart 1991

KÖNIG, J . Athletics and Literature in the Roman Empire, Cambridge/N.Y. 2005

KRAMER, K. Studien zur griechischen Agonistik nach den Epinikien Pindars, Köln 1970

KRATZMÜLLER, B. Synoris - Apene. Zweigespannrennen an den Großen Panathenäen, in: Nikephoros 6, 1993, 75-91

KRATZMÜLLER, B./ Sport and the Construction of Identities, Wien 2007

MARSCHIK, M./

MÜLLER, R. u. a.

(Hrsg.)

KRAUSE, J.H.

Die Gymnastik und Agonistik der Hellenen, Leipzig 1841 (ND Wiesbaden 1971)

KROCKOW, Ch. v. Sport. Eine Soziologie und Philosophie des Leistungsprinzips, Hamburg 1974 
KROPPEN, T.

KRÜGER, A.

KRÜGER, A.

KRÜGER, M.

KRÜGER, A.

KRÜGER, A./

MC CLELLAND, J.

(Hrsg.)

KÜNZL, E.

KUHNEN, H.-P. (Hrsg.)

KUNKEL, W.

KYLE, D. G.
Mortis dolorisque contemptio: Athleten und Gladiatoren in Senecas philosophischem Konzept, (=Nikephoros Beihefte, Bd. 15), Hildesheim 2008 ,Vom Ritual zum Rekord'. Auf dem Weg zur Sportleistungsgesellschaft, in: H. SARKOWICZ (Hrsg.), Schneller, 82-95

Schwimmen. Der Wandel in der Einstellung zu einer Form der Leibesübungen, in:

A. KRÜGER/J. Mc CLELLAND, J. (Hrsg.), Anfänge, $19 \mathrm{ff}$.

On the Limitations of Eichberg's and Mandell's Theory of Sports and Their Quantification in View of Chikaraishi, in: Stadion 3, 1977, 241-252 $=$ J. M. CARTER/A. KRÜGER (Hrsg.), Ritual, 103-114 Einführung in die Geschichte der Leibeserziehung und des Sports. Teil 1: Von den Anfängen bis ins 18. Jahrhundert, Schorndorf 2004

The Ritual in Modern Sport: A Sociobiological Approach, in: J. M. CARTER/A. KRÜGER (Hrsg.), Ritual, 135-151

Die Anfänge des modernen Sports in der Renaissance, London 1984

Der römische Triumph. Siegesfeiern im antiken Rom, München 1988

Morituri. Menschenopfer. Todgeweihte. Strafgerichte (=Schriftenreihe des Rheinischen Landesmuseums Trier 17) Trier 2000 Auctoratus, in: Eos 48, 3, 1957, 207-229 Athletics in Ancient Athens, (Mnemosyne Suppl.95) Leiden/New York/Boston 1993 
KYLE, D. G.

Directions in Ancient Sport History, in: JSH 10, 1983, 7-34

KYLE, D. G.

Games, Prizes and Athletes in Greek Sport: Patterns and Perspectives (1975-1997), in: CB 74.2, 1998, 103-127

KYLE, D. G.

Sport and Spectacle in the Ancient World, Oxford 2007

KYLE, D. C.

The First Hundred Olympiads: A Process of Decline or Democratization, in: Nikephoros 10, 1997, 53-75

KYLE, D. C. The Only Woman in All Greece: Kyniska, Agesilaos, Alcibiades and Olympia, in: JSH 30, 2003, 183-203

KYLE, D. G. Fabulous Females and Ancient Olympia, in: G. P. SCHAUS/S. R. WENN (Hrsg.), Onward, 131-152

KYLE, D. G. Pan-Hellenism and Particularism: Herodotus on Sport, Greekness, Piety and War, in: Z. PAPAKONSTANTINOU (Hrsg.), CulturesSport in the Cultures, 35-63

LA REGINA, A. Nike: il gioco e la vittoria, Mailand 2003

LAFAYE, G. Gladiator, in: D-S 2 (1898), 1563-1599

LAFAYE,G. Circus, in: D-S 1 (1887), 1187-1201

LÄMMER, M.

LÄMMER, M. Die Bedeutung epigraphischer Zeugnisse für die Geschichte der griechischen Gymnastik und Agonistik, Köln 1968

Griechische Wettkämpfe in Jerusalem und ihre politischen Hintergründe, in: KBSW 2, 1973, 182-227

LÄMMER, M. Die Kaiserspiele von Caesarea im Dienste der Politik des Königs Herodes, in: KBSW 3, 1974, 95-164

LÄMMER, M. Olympien und Hadrianeen im antiken Ephesos, Köln 1967

LÄMMER, M.

Paradeuo bei gymnischen Agonen, in: ZPE 1, 1967, 105-106 
LÄMMER, M.

LÄMMER, M.

LANA, 1 .

LANG, A.

LANGNER, M.

LARMOUR, D. H. J .

LAUSCHKE, G.

LEADER-NEWBY, R./

NEWBY, Z. (Hrsg.)

LEBEK, W. D.

LEE, H. M.

LEE, H. M.

LEE, H. M.
Der sogenannte olympische Friede in der griechischen Antike, in: Stadion 8-9, 1982-1983, 4783

Zum Verhalten von Zuschauern bei Wettkämpfen in der griechischen Antike, in: G. SPITZER/D. SCHMIDT (Hrsg.), Eigenständigkeit, 75-85

I ludi capitoli di Domiciano, in: RFIC 29, 1951, 145160

Die Gladiatorendarstellungen in der römischen Kunst, Wien 1937

Antike Graffitizeichnungen. Motive, Gestaltung und Bedeutung (=Palilia 11), Wiesbaden 2001

Stage and Stadium. Drama and Athletics in Ancient Greece (=Nikephoros Beihefte, Bd. 4), Hildesheim 1999

Schriftenverzeichnis von Wolfgang Decker, in Nikephoros 19, 2006, 111-134

Art and Inscriptions in the Ancient World, Cambridge u. a. 2007

Standeswürde und Berufsverbot unter Tiberius: Das ,Senatus Consultum' der Tabula Larinas, in: ZPE 81, 1990, 37-96

The Later Greek Boxing Glove and the "Roman" Caestus: A Centennial Reevaluation of Jüthner's 'Über Antike Turngeräthe', in: Nikephoros 10, 1997, 161-178

The Program and Schedule of the Ancient Olympic Games, Hildesheim 2001

The Halma: A Running or Standing Jump?, in: G. P. SCHAUS/S. R. WENN (Hrsg.), Onward, 153-165 
LEHMANN, St.

LEIBUNDGUT, A.

LENK, $\mathrm{H}$.

LENK, $\mathrm{H}$.

LENK, $\mathrm{H}$.

BEYER, E. (Hrsg.)

LEPPER, F.A.

LETZNER, W.

LEVINSON, D./

CHRISTENSEN, $\mathrm{K}$.

(Hrsg.)

LIM, R.
LENK, H./MOSER, S./

LEVACK, B. P. (Hrsg.)

Zwischen allen Stühlen? Zur Archäologie des antiken Sports, in: Nikephoros 17, 2004, 27-43

Die römischen Lampen in der Schweiz. Eine kulturund handelsgeschichtliche Studie, Bern 1977

Aktuelle Probleme der Sportphilosophie, Schorndorf 1983

Social Philosophy of Athletics, Champaign 1979

Auf der Suche nach dem Wesen des Sports, in: Sportwissenschaft 12, 1982, 202-213

Philosophie des Sports, Schorndorf 1973

Some Reflections on the ,Quinquennium Neronis', in: JRS 67, 1957, 95-103

Der römische Circus: Massenunterhaltung im Römischen Reich, Mainz 2009

The Witchcraft Sourcebook, New York 2004

Encyclopedia of World Sport: From Ancient Times to the Present, Oxford 1996

In the ,Temple of Laughter.' Visual and Literary Representations of Spectators at Roman Games, in:

B. BERGMANN/C. KANDOLEON (Hrsg.), Spectade, 343-365

LINTOTT, A. W. Violence in Republican Rome, Oxford 1968

LOVATELLI Di due rilievi gladiatorii, in: MDAI (R) 15, 1900, 99CAETANI, E. 107

LOVATELLI Di un frammento marmoreo con rilievi gladiatorii, in: CAETANI, E. LOVATELLI BCAR 23, 1895, 253-279

La iscrizione di Crescente auriga circense, in: Antichi CAETANI, E.
Monumenti Illustrati, Rom 1889, 1-21 
LUKAS, G.

LUKAS, G.

LÜSCHEN, G./WEIS, K. $\quad$ Die Soziologie des Sports, Darmstadt 1976

(Hrsg.)

LYLE, E.B.

Mc CLELLAND, J .

Mc CLELLAND, J .

Mc CLELLAND, J .

MC DONNELL, M.

Mc LEOD, W. E.

MC MULLEN, R.

MÄHL, E.

MANCIOLI, D.

MANDELL, R. D.

MANDELL, R. D.

MANDELL, R. D.
Die Körperkultur in frühen Epochen der Menschheitsentwick/ung, Berlin 1969

Der Sport im alten Rom, Berlin 1982

The Circus as a Cosmos, in: Latomus 43, 1984, 827841

Ball Games. From the Roman Gentleman to the Renaissance Warrior, in: The European Sports History Review 5, 2003, 46-64

Body and Mind. Sport in Europe from the Roman Empire to the Renaissance, London/New York 2006 Leibesübungen in der Renaissance und die Freien Künste, in: A. KRÜGER/J. Mc CLELLAND (Hrsg.), Anfänge, 85-110

The Introduction of Athletic Nudity: Thucydides, Plato and the Vases, in: JHS 111, 1991, 182-193 The Range of the Ancient Bow, in: Phoenix 19, 1965, 1-14

Judical Savagery in the Roman Empire, in: Chiron 16, 1986, 147-166

Gymnastik und Athletik im Denken der Römer, Amsterdam 1974

Giochi e spettacoli, Rom 1992

On the Limitations. A Reply to Krüger and Ito, in: Stadion 3, 1977, 257

Sport. Eine illustrierte Kulturgeschichte, München 1986

The Invention of the Sports Record, in: Stadion 2, 1976, 250-264 
MANGAN, J. A.

MANIERI, A.

MANN, C.

MANN, C.

MANODORI, A.

MANTAS, $K$.

MARÓTI, E.

MARÓTI, E.

MARÓTI, E.

MARÓTI, E./

MARÓTI, G.

MARROU, H.-I.

MATTHEWS, V.

MATTHEWS, V. J .

MATTHEWS, V. J .

MATTHEWS, V. J .
Ethnicity, Sport, Identity; Struggle for Status, London [u. a.], 2004

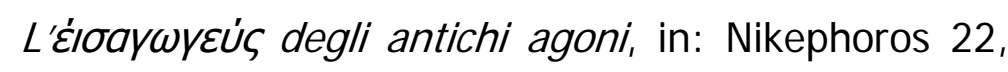
2009, 103-128

Athlet und Polis im archaischen und frühklassischen Griechenland, Göttingen 2001

Gladiators in the Greek East: $A$ Case Study in Romanization, in: Z. PAPAKONSTANTINOU (Hrsg.), Cultures, 124-149

Anfiteatri, circhi e stadi di Roma, Rom 1982

Woman and Athletics in the Roman East, in: Nikephoros 8, 1995, 125-144

Olympiasieger beider Altersgruppen in der Zeit des Aristoteles, in: Nikephoros 18, 2005, 127-135

Zum Siegerepigramm des Nikoladas, in: Nikephoros 3, 1990, 133-140

ПEPIOLONIKH $\Sigma$, in: AAA 31, 1985-1988, 335-355

Zur Frage des Pentathlon-Sieges, in: Nikephoros 6, 1993, 53-59

Geschichte der Erziehung im klassischen Altertum, München $1976^{7}$

Olympic Losers: Why Athletes Who Did Not Win at Olympia are Remembered, in:

G. P. SCHAUS/S. R. WENN (Hrsg.), Onward, 81-93

Suram dare: A Gesture in Roman Ball Playing, in: Nikephoros 3, 1990, 185-187

The Greek Pentathlon again, in: ZPE 100, 1994, 129138

The Hemerodromoi. Ultra Long-Distance Running in Antiquity, in: CW 68, 1974, 161-169 
MATTHEWS, V. J.

MATZ, D.

MATZ, D.

MAU, A.

MAURITSCH, P.

MAURITSCH, P./

PETERMANDL, $R$. (Hrsg.)

MECHIKOFF, R.

MEER, L. B. van der
Sulla and the Games of the $175^{\text {th }}$ Olympiad (80 B. C.), in: Stadion 5.2, 1979, 239-143

Charioteers and Gladiators: Some Comparisons Based on the Epigraphical Evidence, in: CB 56, 1980, 37-39

Greek and Roman Sport. A Dictionary of Athlets and Events from the Eighth Century B.C. to the Third Century A. D., Jefferson, North Carolina/London 1991

Iscrizioni gladiatori di Pompei, in: MD Al (R) 15, 1890, 25-39

Materialien zur Darstellung von Gewalt in literarischen Schilderungen von Kampfsportszenen, in: Nikephoros 19, 2006, 57-66

Antike Lebenswelten: Konstanz, Wandel, u. a. Wirkungsmacht; Festschrift für Ingomar Weiler zum 70. Geburtstag, Wiesbaden 2008

A History and Philosophy of Sport and Physical Education: From the Ancient Greeks to the Present, Madison 1993

Ludi scenici et gladiatorum munus. - A Terracotta Arula in Florence, in: BA Besch 57, 1982, 87-97, 2 Taf.

MEHL, E. Antike Schwimmkunst, München 1927

MEHL, E. Schwimmen, in: RE 5 Suppl. (1931), 847-864

MEHL, E.

MEHL, E.

MEIER, Ch.
Turnkunst, in: RE 7 A (1948), 2513-2556

Warum verzeichneten die Hellenen keine Höchstleistungen bei ihren Wettkämpfen, in: Die Körpererziehung (Schweiz) 25, 1947, 135-142 Das große Fest zu Olympia im Klassischen Altertum, in: Nikephoros 6, 1993, 61-74 
MEIER, M.

MEIER, P. J .

MEIER, P. J.

MENDNER, $\mathrm{S}$.

MENDNER, S.

MENDNER, S.

MENDNER, $\mathrm{S}$.

MERKELBACH, R.

MERKELBACH, R.

MERKELBACH, R.

MERKELBACH, R.

MERKELBACH, R./

STAUBER, J. (Hrsg.)

MERTEN, E. W.

MEZÖ, F.

MEZÖ, F.

MIE, F.
Die Abschaffung der venationes durch Anastasios im Jahr 499 und die ,Kosmische' Bedeutung des Hippodroms, in: H. BECK (Hrsg.), Feiern, 203-232 Ein Gladiatorenrelief des Museums zu Triest, in: MDAl (A) 15, 1890, 162-166

De gladiatura romana quaestiones selectae, Bonn 1881

Das Ballspiel im Leben der Völker, Münster 1956 Boxhandschuhe im Altertum, in: Gymnasium 60, 1953, 20-26

Gesellschaftsspiele, in: RLAC 10, 1978, 847-895

Das Spiel Phaininda-Harpastum, in: Gymnasium 66, 1959, 517-524

Agonistisches Epigramm aus Aphrodisias, in: ZPE 49, 1982, 282-283

Über ein ephesisches Dekret für einen Athleten aus Aphrodisias und über den Athletentitel ПAPA $\triangle O=O \Sigma$, in: ZPE 14, 1974, 91-96

Der griechische Wortschatz und die Griechen, in: ZPE 18, 1975, 101-148

Der Sieg im Pentathlon, in: ZPE 11, 1973, 261-269

Steinepigramme aus dem griechischen Osten, 5 Bde., München/Leipzig 1998-2004

Zwei Herrscherfeste in der Historia Augusta (=Antiquitas IV 5), Bonn 1968

Geschichte der Olympischen Spiele, München 1930 Das Rätsel des altgriechischen Weitsprungs, in: Altertum 4, 1958, 165-172

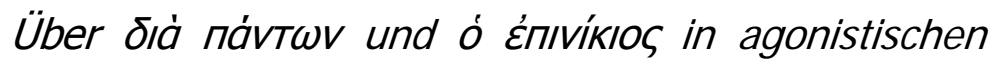
Inschriften, in: MDAl (A) 34, 1909, 1-22 
MIKROGIANNAKIS, E. Olympic Democracy. Pericles' Funural Oration Revisited, in: Nikephoros 21, 2008, 133-157

MILLER, S. G. Anchient Greek Athletics, New Haven/London 2004

MILLER, S. G. Arete. Ancient Writers, Papyri, and Inscriptions on the History and Ideals of Greek Athletics and Games, Chicago 1979

MILLER, S. G. Turns and Lanes in the Ancient Stadium, in: Al A 84, 1980, 159-166

MITSOS, M. Eine agonistische Inschrift aus Argos, in: MD Al (A) 65, 1940, 47-56

MOELLER, W. O. The Riot of A.D. 59 at Pompeii, in: Historia 19, 1970, 84-95

MOMMSEN, A. Feste der Stadt Athen im Altertum, Leipzig $1898^{2}$

MONTGOMERY, H. C: $\quad$ Periodonikes, in: RE 19 (1937), 813-815

MORETTI, L. Iscrizioni agonistiche greche, Rom 1953

MORETTI, L.

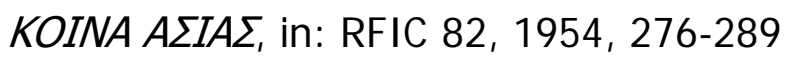

MORETTI, L. Note sugli antichi periodonikai, in: Athenaeum 32, 1954, 115-120

MORETTI, L. Nuovo supplemento al catalogo degli olympionikai, in: MGR 39, 1987, 67-91

MORETTI, L. Olympionikai. I vincitori negli antici agoni olimpici, Rom 1957

MORETTI, L. Supplemento al catalogo degli olympionikai, in: Klio 52, 1970, 295-303

MORGAN, C. Athletes and Oracles: The Transformation of Olympia and Delphi in the Eighth Century B. C., Cambridge 1990

MOSCI SASSI, M. G. I/ linguaggio gladiatorio, Bologna 1992

MOURATIDES, J.

The Origin of Nudity in Greek Athletics, in: JSH 12, 1985, 213-232 
MOURATIDIS, J .

MOURATIDIS, J .

MOURATIDIS, J .

MOURATIDIS, J .

MOURATIDIS, J.

MÜLLER, S.

MÜLLER, S.

MURRAY, S. R./

SANDS, W. A./

KECK, N. A./

O'ROARK, D. A.

MUSTILLI, D.

MUTH, R.

MUTH, R.

Mc CLELLAND, J .
Alexander The Great and His Promotion of Greek Games in the East, in: CJ HSP 13.1, 1982, 61-73

On the Jump of the Ancient Pentathlon, (=Nikephoros Beihefte 20), Hildesheim 2012

The Length of the Running Race in the Ancient Pentathlon, in: Nikephoros 23, 201,, 57-69

Nero: The Artist. The Athlete and his Downfall, in: JSH 12.1, 1985, 5-20

On the Origin of the Gladiatorical Games, in: Nikephoros 9, 1996, 111-134

"Schauspiele voller Kraft und Charakter". Die Gladiatorenkämpfe als Drama fürs Volk, in: Gymnasium 109, 2002, 21-47

Das Volk der Athleten. Untersuchungen zur Ideologie und Kritik des Sports in der griechischrömischen Antike (=Bochumer Altertumswissenschaftliches Colloquium Bd. 21), Trier 1995

Efficacy of the Ankyle in Increasing the Distance of the Ancient Greek Javelin Throw, in: Nikephoros 23, 2010, 43-55

Nike: ideologia, iconografia e feste della vittoria in età antica, Rom 2005

Olympia - Idee und Wirklichkeit, in: Serta Philologica Aenipontana 3, 1979, 161-202

Der Sieg zu Olympia: Faszination und Kritik, in: Wort im Gebirge 15, 1976, 7-39

The Numbers of Reason: Luck, Logic, and Art in Renaissance Conceptions of Sport, in: J. M. CARTER/A. KRÜGER (Hrsg.), Ritual, 53-64 
NEILS, J . (Hrsg.)

Goddess and Polis. The Panathenaic Festival in Ancient Athens (Ausstellungskatalog), Hannover/New Hampshire 1992

NELSON, M. The First Olympic Games, in: G. P. SCHAUS/S. R. WENN (Hrsg.), Onward, 47-68 NEUMANN-HARTMANN, Prosopographie zu den Epiniken von Pindar und A. Bakchylides, in: Nikephoros 21, 2008, 81-131

NEUMANN-HARTMANN, Das Wettkampfprogramm der panhellenischen Spiele A. im 5. Jh. V. Chr., in: Nikephoros 20, 2007, 113-151

NEUMANN-

HARTMANN, A. Epinikien und ihr Aufführungsrahmen, Hildesheim NEWBOLD, R. F. 2009

Dio Cassius and the Games, in: AC 44, 1975, 589604

NEWBY, Z. Greek Athletics as Roman Spectacle: The Mosaics from Ostia and Rome, in: PBSR 70, 2002, 177-203

NEWMYER, St. T. Animals in Greek and Roman Thought, London 2011

NIBLEY, $\mathrm{H}$.

Sparsiones, in: CJ 12, 1944-45, 515-543

NICOLAl, $\mathrm{K}$. Feiertage und Werktage im römischen Leben, besonders in der Zeit der ausgehenden Republik und in der frühen Kaiserzeit, in: Saeculum 14, 1963, 194220

NIELSEN, T. H.

NILSSON, M. P. Olympia and the Classical Helenic City-State Culture, Kopenhagen 2007 (=Historiske-filosofiske Meddelslser 96)

NIMAS, T. Griechische Feste, Leipzig 1906 (ND Stuttgart 1957) The Athletic Games as a Criterion for the Choice of a Husband in Greece. Myth and Reality, in: Nikephoros 13, 2000, 221-240

NITSCHKE, A. Kunst und Verhalten. Analoge Konfigurationen (=problemata 40), Stuttgart-Bad Cannstatt 1975 
NOGALES

BASARRATE, T. (Hrsg.) Mérida 2002

OLIVER, J. H.

OLIVER, J. H./

PALMER, E. A.

OLIVOVÁ, A.

OLIVOVÁ, V.

OLIVOVÁ, V.

ORLANDI, S.

ORTKEMPER, $\mathrm{H}$.

PALMIERI, R.

PAPAKONSTANTINOU,

Z. (Hrsg.)

PAPAKONSTANTINOU,

Z. (Hrsg.)

PAPALAS, A. J.

PAPALAS, A. J.

PAPAPOSTOLOU, I. A.

PAPINI, M. 67-76
Ludi Romani. Espectáculos en Hispania Romana, Symmachi, homo felix, in: MAAR 25, 1957, 7-16 Minutes of an Act of the Roman Senate, in: Hesperia 24, 1955, 320-343

Sport und Spiel im Altertum.Eine Kulturgeschichte München 1985

Chariot Racing in the Ancient World, in: Nikephoros 2, 1989, 65-88

Kalokagathia - The Greek Ideal of the Harmonious Personality, in: CJHSP 14.2, 1983, 1-15

Olympia. Mythos und Geschichte moderner Wettkämpfe, Wien 1980

Olympische Legenden: Geschichten aus dem antiken Olympia, Frankfurt a. M. 1996

Ricordi di ludi circenses a Teanum Sidicinum, in: RA AN 53, 1978, 57-65

Epilogue: Fresh Perspectives on Ancient Sport, in: Z. PAPAKONSTANTINOU (Hrsg.), Cultures, 217-219 Sport in the Cultures of the Ancient World.New Perspectives, London/New York 2010 Boy Athletes in Ancient Greece, in: Stadion 17.2, 1991, 165-192

The Development of Greek Boxing, in: AW 9, 1984,

Monuments des combats de gladiateurs à Patras, in: BCH 113, 1989, 351-401

Munera gladiatoria e venationes nel mondo delle imagini, Rom 2004 
PAPPAS, C.

PARKERSON, D.

PARLASCA, K.

PATRUCCO, R.

PATRUCCO, R.

PAUSZ, R.-D./

REITINGER, W.

PAVLOGI ANNIS, O./

ALBANIDIS, E./

PEARSON, J.

PEEK, $W$.

PEEK, W.

PEEK, W.

PEIM, O.

PEISER, B. J .
DIMITRIOU, $M$.

Die antike agonale Idee und ihre Nachwirkungen auf das heutige griechische Leben, Diss. Saarbrücken 1980

The New Sport History, in: J. M. CARTER/A. KRÜGER (Hrsg.), Ritual, 13-20

Die römischen Mosaiken in Deutschland, Berlin 1959 La psicologia dell'atleta, in: Maia 23, 1971, 245-253

Lo sport nella Grecia antica (=Arte e Archeologia. Studi e documenti), Florenz 1972

Das Mosaik der gymnischen Agone von Batten Zammour, Tunesien, in: Nikephoros 5, 1992, 119123

The Aktia of Nikopolis: New Approaches, in: Nikephoros 22, 2009, 79-102

Arena. The Story of the Colosseum, London 1973 Delische Gedichte, in: WZ Halle 6, 1956-1957, 572 Delphische Gedichte, in: MDAl (A) 67, 1942, 232269

Griechische Vers-Inschriften, I. Grab-Epigramme, Berlin 1955

Die Vorliebe des Pausanias für den Kampfsport, in: Nikephoros 13, 2000, 95-109

Das dunkle Zeitalter Olympias. Kritische Untersuchung der historischen, archäologischen und naturgeschichtlichen Probleme der griechischen Achsenzeit am Beispiel der antiken Olympischen Spiele, Frankfurt a. M. 1993

PERROT, S.
Les premiers concours des Pythia, in: Nikephoros 22, 2009, 7-13 
PERROTTET, T.

PETERMANDL, $W$.

PETERMANDL, W.

The Naked Olympics: The True Story of the Ancient Games, New York 2004

Noch einmal zum ,Zuschauerkrawall' von Pompeji, in: MAURITSCH, P./ PETERMANDL, R. u. a. (Hrsg.), Antike Lebenswelten, 179-189

Überlegungen zur Funktion der Altersklassen bei den griechischen Agonen, in: Nikephoros 10, 1997, 135147

PETZL, G.

Die Inschriften von Smyrna (=IK 23-24), Bonn 19821990

PETZL, G. Gladiatoren - Denkmäler aus den Skizzenbüchern von Josef Keil, in: ZPE 14, 1974, 285-293

PFEIJ FFER, I. L. Athletic Age Categories in Victory Odes, in: Nikephoros 11, 1998, 21-38

PFEILSCHIFTER, R. Republikanische Feste und Sinnstiftung durch aitiologischen Mythos, in: H. BECK (Hrsg.), Feiern, 109-140

PFISTER, G.

Die Erneuerung der römischen iuventus durch Augustus, Bochum 1977

PFUHL, E./MÖBIUS, H. Die ostgriechischen Grabreliefs, 2 Bde., Mainz 1979

PIERNAVIEJA, P. Corpus des inscriptiones deportivas de la España romana, Madrid 1977

PLASS, P. C. The Game of Death in Ancient Rome: Arena Sport and Political Suicide, Madison (Wisconsin) 1995

PLEKET, H. W. Athleten im Altertum. Soziale Herkunft und Ideologie, in: Nikphoros 18, 2005, 151-163

PLEKET, H. W. Einige Betrachtungen zum Thema , Geld und Sport', in: Nikephoros 17, 2004, 77-89

PLEKET, H. W. Games, Prizes, Athletes and /deology, in: Stadion 1, $1975,49-89$ 
PLEKET, H. W.

PLEKET, H. W.

PLEKET, H. W.

PLEKET, H. W.

PLEKET, H. W.

PLEKET, H. W.

POLIAKOFF, M.

POLIAKOFF, M.,

POLJ AKOV, F. B.

POPPLOW, U.

POTTER, D.

POTTER, D./

MATTINGLY, D.

PREUNER, E.

PRITCHARD, D. M.

RAMBA, D.
Olympic Benefactors, in: ZPE 20, 1976, 1-18

Some Aspects of the History of the Athletic Guilds, in: ZPE 10, 1973, 197-227

Zur Soziologie des antiken Sports, in: MNIR 36, 1974, 57-87

Zur Soziologie des antiken Sports, in: Nikephoros 14, 2001, 157-212, Addenda 2002, 209-212

Sport und Leibesübungen in der griechischen Welt des hellenistisch-römischen Zeitalters, in: GL 2, 280311

Roman Emperors and Greek Athletes, in: Nikephoros 23, 2010, 175-203

Kampfsport in der Antike, Zürich/München 1989

Studies in the Terminology of Greek Combat Sports, Königstein 1982

Die Inschriften von Tralleis und Nysa (IK 36), Bonn 1989

Leibesübungen und Leibeserziehung in der griechischen Antike, Schorndorf 1959

Death as Spectacle and Subsequent Disposal, in: JRA 14, 2001, 478-484

Life, Death and Entertainment in the Roman Empire, Ann Arbor 1999

Amphiaraia und Panathenaia, in: Hermes 57, 1922, 80-106

Sport, War and Democracy in Classical Athens, in: Z. PAPAKONSTANTINOU (Hrsg.), Cultures, 64-97 Gladiatur - Gegenstand sporthistorischer Betrachtung, in: W. BUSS/A. KRÜGER (Hrsg.), Sportgeschichte, 53-61 
RAMBA, D.

RAMBA, D.

RASCHKE, W. J .

RASCHKE, W. J .

RASCHKE, W. J. (Hrsg.) The Archaeology of the Olympics. The Olympics and

RAUBITSCHEK, A. E. The Agonistic Spirit in Greek Culture, in: Anc W 7,

RAWSON, E.

REA, R./BESTE, H. J./ // cantiere del Colosseo, in: MDAl (R) 109, 2002, LANCASTER, L. C.

REGNER, J .

REGNER, J .

REICHMANN, E.

REIS, M.

REISCH, E.

REMIJ SEN, S.

REYNOLDS, J. M. Other Festivals in Antiquity, Madison (Wisconsin)/London 1988 1983, 3-7

Chariot-Racing in the Roman Republic, in: PBSR 49, 1981, 1-16

Recordmania in Sports in Ancient Greece and Rome, in: J. M. CARTER/A. KRÜGER (Hrsg.), Ritual 31-39 Verdienstmöglichkeiten von Spitzensportlern in der Antike, in: Sozial- und Zeitgeschichte des Sports 4, 1990, 75-83

Aulos and Athlete: The Function of the Flute Players in Greek Athletics, in: Arete 2, 1985, 177-200

A Red-Figure Kylix in Malibu. The Iconography of Female Charioteers, in: Nikephoros 7, 1994, 157-180 341-374

RE Suppl. 7 (1940), s. v. Ludi circenses, 1626-1664

RE 18 (1939), s. v. Olympionikai, 232-241

Herrschaft der Zahl. Quantitatives Denken in der deutschen Aufklärung, Stuttgart 1968

Sport bei Horaz, (Nikephoros Beihefte I, 2), Hildesheim 1994

RE 2 (1896), s. v. Athletai, 2049-2058

Challenged by Egyptians: Greek Sports in the Third Century B.C., in: Z. PAPAKONSTANTINOU (Hrsg.), Cultures, 98-123

New Letters from Hadrian to Aphrodisias: Trials, Taxes, Gladiators and an Aqueduct, in: JRA 13, $2000,5-20$ 
RICCI, C.

RICHLIN, A. (Hrsg.)

RICL, M.

RIEGER, B.

RIGSBY, K. J.

RINGWOOD, I. C

ROBERT, L.

ROBERT, L.

ROBERT, L.

ROBERT, L.

ROBERT, L.

ROBERT, L.

ROBERT, L.

ROBERT, L.

ROBERT, L.

ROBERT, L.
Gladiatori e attori nella Roma giulio-claudia: studi sul senatoconsulto di Larino, Mailand 2006

Pornography and Representation in Greece and Rome, New York/Oxford 1992

The Inscriptions of Alexandria Troas (=IK 56), Bonn 1997

Die Kapitolia des Kaiser Domitian, in: Nikephoros 12, 1999, 171-203

A Victory List from Cos, in: Nikephoros 23, 2010, 171-174

Agonistic Features of Local Greek Festivals Chiefly from Inscriptional Evidence, Columbia 1927

Catalogue agonistique des Romaia de Xanthos, in: RA 1978, 277-290

Deux concours grecs à Rome, in: CR Al 1970, 6-27

Deux inscriptions agonistiques de Rhodes, in: AE 1966, 108-118

Enterrements et épitaphes, in: AC 37, 1968, 406-448 Les épigrammes satiriques de Lucillius sur les athlètes: parodie et réalités, in: Entretiens sur I'Antiquité Classique. Bd. 14. L'épigramme grecque. Genf (Fondation Hardt) 1968, 181-291 Études anatoliennes, Paris 1937 (ND Amsterdam 1970)

Études épigraphiques et philologiques, Paris 1938 Les gladiateurs dans l'Orient grec, Paris 1940 (ND Amsterdam 1971)

Inscription agonistique d'Ancyre concours d'Ancyre, in: Hellenica 11-12, 1960, 350-368

Sur des inscriptions d'Ephèse, in: RPh 41, 1967, 785 
ROBERT, L.

ROBERT, L.

ROBERT, L.

ROBERT, L.

ROBERT, L.

ROBERT, L.

ROBERT, L.

ROCCHEITI, L.

ROUCHÉ, Ch.

ROMANO, D. G.

ROMANO, M.

ROOS, P.

RUDOLPH, W.
Inscriptions grèques d'Asie mineure, in: Anatolian Studies presented to W. H. Buckler, Manchester 1939, 227-248

Monuments des gladiateurs dans /'Orient grec, in: Hellenica 3, 1946, 112-150

Monuments des gladiateurs dans /'Orient grec, in: Hellenica 5, 1948, 77-99

Monuments des gladiateurs dans /'Orient grec, in: Hellenica 7, 1949, 126-151

Monuments des gladiateurs dans /'Orient grec, in: Hellenica 8, 1950, 39-72

Opera minora selecta: épigraphie et antiquités grècques, 7 Bde., Amsterdam 1969-1990

PYKTEYEIN, in: RA 31, 1929, 24-41

// mosaico con scene d'arena al Museo Borghese, in: RIA 10, 1961, 29-115

Performers and Partisans at Aphrodisias in the Roman and Late Roman Periods: A Study Based on Inscriptions from the Current Excavations at Aphrodisias in Caria, (=Society for the Promotion of Roman Studies), London 1993

Judges and Judging at the Ancient Olympic Games, in: G. P. SCHAUS/S. R. WENN (Hrsg.), Onward, 95113

L'épigraphe atenièse à Phayllos (IG, $\mathrm{I}^{3} 2$, 823), in: ZPE 123, 1998, 105-116

The Start of the Greek Foot Race, in: OAth 6, 1965, 149-156

Antike Sportgeräte, in: Klio 48, 1967, 81-92 
RUDOLPH, W.

RÜHL, J . K.
RUDOLPH, W.

Zu Formen des Berufssports zur Zeit der Poliskrise, in: E. Ch. WELSKOPF (Hrsg.), Poleis, Bd. 3, 14721483

Olympischer Kampfsport in der Antike, Berlin 1965

Wesen und Bedeutung von Kampfansagen und Trefferzählskizzen für die Geschichte des spätmittelalterlichen Turniers, in: G. SPITZER/

D. SCHMIDT (Hrsg.), Eigenständigkeit, 86-112

RÜPKE, J./MÖLLER, A. In: DNP 12, s. v. Zeitrechnung, 2002, 717-724

SABBATINI

Epigrafia anfiteatrale dell'Occidente Romano, I.

TUMOLESI, $\mathrm{P}$. Roma (=Vetera 2), Rom 1988

SABBATINI Gladiatorum paria, Rom 1980

TUMOLESI, $\mathrm{P}$.

SANFILIPPO, C.

Gli 'Auctorati', in: Studi in Onore die Arnaldo Biscardi, Bd. 1, Mailand 1982, 181 - 192

SANSONE, D. Greek Athletics and the Genesis of Sport, Berkeley/Los Angeles/London 1988

SARKOWICZ, H. (Hrsg.) Schneller, Höher, Weiter, Eine Geschichte des Sports, Frankfurt a. M./Leipzig 1996

SAVI, F. I gadiatori. Storia, organizzazione, iconografia, Rom 1980

SAYLOR, Ch. Funural Games: the Significance of Games in the Cena Trimalchionis, in: Latomus 46, 1987, 593-602

SCANLON, Th. F. Contesting Ancient Mediterranean Sport, in: Z. PAPAKONSTANTINOU (Hrsg.), Cultures, 1-12

SCANLON, Th. F. Eros and Greek Athletics, Oxford/New York 2002 SCANLON, Th. F. Greek and Roman Athletics. A Bibliography, Chicago 1984

SCANLON, Th. F. Greek Boxing Gloves: Terminology and Evolution, in: Station 8-9, 1982-1983, 31-45 
SCHAUENBURG, K. Zirkusrennen und verwandte Darstellungen, Berlin 1995

SCHAUMBERG, A. Bogen und Bogenschießen bei den Griechen, Nürnberg 1910

SCHAUS, G. P./ WENN, Onward to the Olympics. Historical Perspectives on

S. R. (Hrsg.)

SCHIROK, E.

SCHMID, D.

SCHMID, H.

SCHMIDT, S.

SCHNEIDER, $K$.

SCHOLZ, U. W.

SCHROEDER, B.

SCHWERTHEIM, E.

SCOBIE, A.

SCULLARD, H. H.

SEGAL, E.

SEGRAVE, J. O./CHU, D. The Olympic Games in Transition, Champaign 1988

the Olympic Games, Waterloo (Kanada) 2005

Gladiatorenspiele. Die Amphitheater von Vienne. Orange und Arles, in: AU 31, 1988, 52-68

Die römischen Mosaiken aus Augst und Kaiseraugst (=Forschungen in Augst 17), Augst 1993

Zur Technik des Weitsprungs (äג $\mu a)$ in der griechischen Antike. Eine Neubewertung literarischer und bildlicher Quellen unter Berücksichtigung biomechanischer Bewegungsanalysen und volkskundlicher Tradition, Mainz 1997

Mark Aurel und Spectacula, in: Stadion 10, 1984, 21-43

RE Suppl. 3 (1918), s. v. Gladiatores, 760-784

Der römische Kalender: Entstehung und Entwicklung, Stuttgart 2011

Der Sport im Altertum, Berlin 1927

Die Inschriften von Kyzikos und Umgebung (=IK 18, 26), Bonn 1980-83

Spectator Security and Comfort at Gladiatorial Games, in: Nikephoros 1, 1988, 191-243

Römische Feste. Kalender und Kult (=Kulturgeschichte der antiken Welt, Band 25), Mainz 1985

To Win or to Die of Shame. A Taxonomy of Values, in: JSH 11.2, 1984, 25-31 
SEMMELINGER, L.

SHEAR, J . L.

SIEBLER, M.

SIEFERLE,

BREUNI NGER,

(Hrsg.)

SIMON, H.-G.

SIMONS, B.

SINN, U.

SINN, U.

R. P./ Kulturen der Gewalt: Ritualisierung und H. Symbolisierung von Gewalt in der Geschichte, Frankfurt a. M./New York 1988

Zwei außergewöhnliche reliefverzierte Gefäße aus Langenhain, Wetteraukreis, in: Germania 53, 1975, 126-137, Taf. 35-38

Cassius Dio und die Römische Republik: Untersuchungen zum Bild des römischen Gemeinwesens in den Büchern 3-35 der Rōmaïka, Berlin u. a. 2009

Das antike Olympia: Götter, Spiel und Kunst, München 2004

Olympia. Kult, Sport und Fest in der Antike, München 1996

SLATER, W. J. (Hrsg.) Roman Theater and Society, Ann Arbor 1996

SOMMELLA, A. M. u. a. Lo sport nel mondo antico (Ausstellungskatalog Rom (Hrsg.) 1987), Mailand 1987

SOURVINOUTheseus Lifting the Rock and a Cup Near the Pinthos INWOOD, Ch. SPITZER, G./ SCHMIDT, D. (Hrsg.)

SPIVEY, N. Painter, in: JHS 91, 1971, 94-109

Sport zwischen Eigenständigkeit und Selbstbestimmung: Pädagogische und historische Beiträge aus der Sportwissenschaft. Festschrift für HAJO BERNETT, Bonn 1986 The Ancient Olympics, Oxford 2004 
SPIVEY, N.

STAAB, G.

STAAB, G./PETZL, G. Vier neue Epigramme aus Lydien, in: ZPE 174, 2010,

STEI NER, D.

STEI NKOPF, G.

STERN, E. von

STEUERNAGEL, D.

STEWART, A.

STEWART, R.

STRASSER, J. Y.

STRASSER, J.-Y.

STURZEBECKER, R. L. Athletic-Cultural Archaeological Sites in the GrecoRoman World, Leiden 1985

SUHLING, L. Meßtechnik und Erfahrungswissenschaften, in: R. E. VENTE, (Hrsg.), Erfahrung und Erfahrungswissenschaft, Stuttgart/Berlin/Köln/Mainz 1974, 96-103

SWADDLING, J.

SWEET, W. E.

Understanding Greek Sculpture. Ancient Meanings, Modern Readings, New York 1996

$\mathrm{Zu}$ den neuen Gladiatorenmonumenten aus Stratonikeia in Karien, in: ZPE 161, 2007, 35-46 1-14

Moving Images: Fifth-Century Victory Monuments and the Athlete's Allure, in: Cl Ant 17, 1998, 123-149

Untersuchungen zur Geschichte des Ruhmes bei den Griechen, Halle 1937

Der Pfeilschuß des Olbiopoliten Anaxagoras, in: JÖAl 4( Beibl)., 1901, 57-60

Ritus funebres? Etruskische Bilder mythischer Zweikämpfe und der Ursprung der munera gladiatorum, in: Hephaistos 15, 1997, 69-92

Art, Desire and the Body in Ancient Greece, Cambridge 1997

The Original Olympics, Hove 1996

Les Olympia d'Alexandrie et le pancratiaste M. Aur. Asklepiades, in: BCH 128-129, 2004-2005, 421-468

Un départ de course à pied sure une lamp romaine, in: Nikephoros 23, 2010, 241-266

The Ancient Olympic Games, London $2008^{2}$

Protection of the Genitals in Greek Athletics, in: Anc W 11, 1985, 43-52 
SWEET, W. E.

TABACHOVITZ, D.

TEJ A, A.

TEJA, A.

TEYSSIER, E.

THESENVITZ, U.

THOMAS, R.

THOUVENOT, R.

THUI LLIER, J.-P.

THUILLIER, J.-P.

THUILLIER, J.-P.

THUI LLLER, J .-P.

TOD, M. N.

TOD, M. N.

TOD, M. N.
Sport and Recreation in Ancient Greece, New York/Oxford 1987

Zu den dem Wagenlenker Porphyrios gewidmeten Inschriften, in: Eranos 56, 1958, 159-172

L'esercizio fisico nell'antica Roma, Rom 1988

Sport e culture, 1. Età antica, Rom 2005

La mort en face. Le dossier gladiateurs, Nîmes 2005

Olympia in der Antike. Kommunikationszentrum der griechischen Welt, Münster 1973

Aurigae und Agitatores. $\mathrm{Zu}$ einer Wagenlenkerstatuette im Römisch-Germanischen Museum Köln, in:KJ 34, 2001, 489-522

Sur deux statuettes de gladiateurs du Maroz romain, in: Hommages à Leon Hermann (=Latomus 44), 1960, 715-721

La nudité athlétique (Grèce, Étrurie, Rome), in: Nikephoros 1, 1988, 29-48

Le programme athlétique des ludi circenses dans la Rome républicaine, in: REL 60, 1982, 105-122

Sport im antiken Rom (übers. W. Decker), Darmstadt 1999

Les jeux athlétiques dans la civilisation étrusque (BEFAR 256), Rom 1985

Greek Record-Keeping and Record-Breaking, in: CQ 43, 1949, 105-112

Teams of Ball-Players at Sparta, in: ABSA 10, 19031904, 63-77

Three new $\Sigma \Phi A / P E I$-inscriptions, in: ABSA 13, 1906-1907, 212-218

TÖLLE-KASTENBEIN, R. Pfeil und Bogen im antiken Griechenland, Bochum 1980 
TOYNBEE, J. M. C

TOYNBEE, J. M. C

TRACY, St. V.

TRAVERSARI, G.

TREMEL, J .

TREMEL, J .

TREMEL, J .

TREMEL, J .

UEBERHORST, $\mathrm{H}$.

(Hrsg.)

ULF, C.

ULF, C.

ULF, C./WEI LER, I.

UNGERN-

STERNBERG, J . von
Beasts and Their Names in the Roman Empire, in: PBSR 16, 1948, 24-37

Tierwelt der Antike, (=Kulturgeschichte der Antiken Welt) Mainz 1983

The Panathenaic Festival and Games. An Epigraphic Inquiry, in: Nikephoros 4, 1991, 133-154

Gli spettacoli in acqua nel teatro tardo-antico, Rom 1960

Die Befragung des Orakels durch Athleten, in: Nikephoros 17, 2004, 111-118

Zur Fluchscherbe gegen die Renngesellschaft der Grünen, in: Nikephoros 18, 2005, 179-183

Magica agonistica: Fluchtafeln im antiken Sport, Hildesheim 2004 (=Nikephoros Beihefte 10)

Die Steinzeile in der Laufbahn des Stadions von Olympia (=Nikephoros Beihefte, Bd. 16), Hildesheim 2009

Geschichte der Leibesübungen, Bde. 1-6, Berlin/München/Frankfurt a. M. 1972-1989

Die Einreibung der griechischen Athleten mit Öl, in: Stadion 5.2, 1979, 220-238

Die Mythen um Olympia - politischer Gehalt und politische Intention, in: Nikephoros 10, 1997, 9-51 Der Ursprung der antiken Olympischen Spiele in der Forschung. Versuch eines kritischen Kommentars, in: Stadion 6, 1980, 1-38

Die Einführung spezieller Sitze für die Senatoren bei den Spielen (194 v. Chr.), in: Chiron 5, 1975, 157163 
UNRUH, F.

UNRUH, F. u. a.

VALAVANIS, P.

VANHOVE, D. (Hrsg.)

VASILIEV, A. A.

VENSKE, W.

VERSNEL, H. S.

VEYNE, P.

VEYNE, $P$.

VEYNE, P.

VEYNE, P.

VIERECK, P.

VIEWEG, R.

VIGNERON, P.

VILLE, G.
Totenkult und Todeskämpfe. Religion, Strafe und Rausch im Amphitheater, in: H.-P. KUHNEN (Hrsg.), Morituri, 71-104

Morituri. Menschenopfer, Todgeweihte, Strafgerichte [Ausstellungskatalog], Trier 2000

Games and Sanctuaries in Ancient Greece: Olympia, Delphi, Isthmia, Nemea, Athens, Los Angeles 2004

Le sport dans la Grèce antique: du jeu à la compétition, Brüssel 1992

The Monument of Porphyrius in the Hippodrome of Constantinople, in: DOP 4, 1948, 29-49

Plato und der Ruhm, Würzburg 1938

Triumphus. An Inquiry Into the Origin, Development and Meaning of the Roman Triumph, Leiden 1970 Agustal de l'an 1. - Premier pontarque, in: BCH 90, 1966, 141-155

Brot und Spiele: Gesellschaftliche Macht und politische Herrschaft in der Antike, Frankfurt/Main 1992

Die griechische Religion: Kult, Frömmigkeit und Moral, Stuttgart 2008

Paiens et chrétiens devant la gladiature, in: MEFRA $111,1999,883-917$

Aktenstücke zum griechisch-römischen Vereinswesen, in: Klio 8, 1908, 413-426

Maß und Messen in kulturgeschichtlicher Sicht, Wiesbaden 1962

Le cheval dans l'antiquité gréco-romaine, 2 Bde., Nancy 1968

La gladiature en Occident des origines à la mort de Domitien, Paris/Rom 1981 
VILLE, G.

WACKE, A.

WACKER, C.

WAGNER, E.

WAGNER, E.

WALLNER, Ch.

WATSON, G. R.

WEBER, C. W.

WEBER-HIDEN, I.

WEEBER, K.-W.

WEEBER, K.-W.

WEEBER, K.-W.

WEEBER, K.-W.

WEGNER, E.

WEI CKERT, C.

WEI LER, I.
Les jeux de gladiateurs dans l'empire chrétien, in: MEFRA 72, 1960, 273-335

Athleten als Darlehensnehmer nach römischen Recht, in: SDHJ 44, 1978, 439-452

The Record of the Olympic Victory List, in: Nikephoros 10, 1997, 103-117

Hockeyspiel im Altertum, in: Philologus 103, 1959, 173-140

Kritische Bemerkungen zum Harpastum-Spiel, in: Gymnasium 70, 1963, 356-366

Soldatenkaiser und Sport (=Grazer Altertumskundliche Studien, Bd. 4), Frankfurt a. M 1997

Theta nigrum, in: J RS 42, 1952, 56-62

Brot und Spiele. Massenunterhaltung als Politik im antiken Rom, Herrsching 1989

Wettkampfdarstellungen auf Terra Sigillata, in: Nikephoros 5, 1992, 103-118

Luxus im alten Rom: die öffentliche Pracht, Darmstadt 2006

Panem et circenses. Massenunterhaltung als Politik im Alten Rom, Mainz $1999^{3}$

Die unheiligen Spiele. Das antike Olympia zwischen Legende und Wirklichkeit, Zürich/München 1991 Wahlkampf im alten Rom, Düsseldorf 2007 Das Ballspiel der Römer, Würzburg 1938 Gladiatoren-Relief der Münchner Glyptothek, in: Münchner J ahrbuch der bildenden Kunst, N. F. II. 1/2, 1925, 1-39

Der Agon im Mythos, Darmstadt 1974 
WEI LER, I.

WEI LER, I.

WEILER, I.

WEI LER, I.

WEILER, I.

WEI LER, I.

WEILER, I.

WEI LER, I.

WEI LER, I.

WEILER, I.

WEILER, I.

WEI LER, I. (Hrsg.)

WEI LER, I. (Hrsg.)
Der Agon, die Agonalität und das Agonale aus der Sicht des Althistorikers, in: LSB 49, 2008, 4-26 AIEN APICTEYEIN, Ideologiekritische Bemerkungen zu einem vielzitierten Homerwort, in: Stadion 2, 1975, 199-227

Einige Bemerkungen zu Solons Olympionikengesetz, in: P. HÄNDEL/W. MEID (Hrsg.), Festschrift R. Muth, $1983,573-582$

Langzeitperspektiven zur Genese des Sports, in: Nikephoros 2, 1989, 7-26

Der "Niedergang" und das "Ende" der antiken Olympischen Spiele in der Forschung, GB 12-13, 1985-1986, 235-257

Olympia - jenseits der Agonistik: Kultur und Spektakel, in: Nikephoros 10, 1997, 191-213

Der Sport bei den Völkern der alten Welt, Darmstadt $1988^{2}$

Theodosius I. und die Olympischen Spiele, in: Nikephoros 17, 2004, 53-75

Zum Verhalten der Zuschauer bei Wettkämpfen in der Alten Welt, in: E. Kornexel (Hrsg.), Spektrum der Sportwissenschaft, Festschrift zum 80. Geburtstag von F. Fetz, Wien 1987, 43-59

Der Wettkampf - ein Privileg der Griechen?, in: Wort im Gebirge 15, 1976, 40-54

Wider und für das agonale Prinzip - eine griechische Eigenart?, in: Nikephoros 19, 2006, 81-110

Quellendokumentation zur Gymnastik und Agonistik im Altertum, 7 Bde., Wien u. a. 1991-2002

Soziale Randgruppen und Außenseiter im Altertum, Graz 1988 
WEI SMANN, W.

WELCH, K. E.

WELCH, K. E.

WELCH, K. E.

WELCH, K. E.

WELSKOPF, E.

WELSKOPF, E. (Hrsg.)

WERNER, K.

WIEDEMANN, T.

WIEDEMANN, T.

WIEDEMANN, T.

WIEGARTZ, H.

WIEMER, H.-U.

WILL, W.
Kirche und Schauspiele (=Cassiciacum 27), Würzburg 1972

Greek Stadia and Roman Spectacles: Asia, Athens, and the Tomb of Herodes Atticus, in: JRA 11, 1998, 117-145

The Roman Amphitheatre. From the Origins to the Colosseum, Cambridge 2007

Roman Amphitheatres revived, in: J RA 4, 1991, 272281

The Roman Arena in Late-Republican Italy: a New Interpretation, in: JRA 7, 1994, 59-80

Die Krise des Sports im Spiegel der Literatur und der Philosophie, in: E. WELSKOPF (Hrsg.), Krise, Bd. 4, 1484-1489

Hellenische Poleis:Krise, Wandlung, Wirkung $4 \mathrm{Bd}$. ,Berlin 1974

Römische Lampen und Leuchter. Auswahlkatalog des Rheinischen Landesmuseums Trier, Trier 1997

Adults and Children in the Roman Empire, London 1989

Das Ende der römischen Gladiatorenspiele, in: Nikephoros 8, 1995, 145-159

Kaiser und Gladiatoren. Die Macht der Spiele im antiken Rom, Darmstadt 2001

Zur Startanlage im Hippodrom von Olympia, in: Boreas 7, 1984, 41-78

Neue Feste - neue Geschichtsbilder? Zur Erinnerungsfunktion städtischer Feste im Hellenismus, , in: H. BECK (Hrsg.), Feiern, 83-108 Der römische Mob. Soziale Konflikte in der späten Republik, Darmstadt 1991 
WILLCOCK, M. M.

WISSOWA, G.

WISTRAND, $M$.

WOLICKI, A.

WOLLMANN, $\mathrm{H}$.

WOLOCH, M.

WOLTERS, $\mathrm{P}$.

WOODWARD, A. M.

WOODWARD, A. M.

WÖRRLE, M.

WÜNSCHE, R. (Hrsg.)
Pindar: Victory Odes: Olympians 2, 7, 11; Nemean 4; Isthmians 3, 4, 7. Cambridge 1995 Religion und Kultur der Römer, München $1971^{2}$ Entertainment and Violence in Ancient Rome. The Attidudes of Roman Writers of the First Century A. D., Göteborg 1992

The Heralds and the Games in Archaic and Classical Greece, in: Nikephoros 15, 2002, 69-97

Retiarier - Darstellungen auf römischen Tonlampen, in: MDAl (R) 32, 1917, 147-167

Athenian Trainers in the Aeginetan Odes of Pindar and Bacchylides, in: CW 56, 1963, 102-104

Zu griechischen Agonen, Würzburg 1901

Inscriptiones Graecae 5,1: Some Afterthoughts, in: ABSA 43, 1948, 209-259

Some Notes on the Spartan spaipei', in: ABSA 46, 1951, 191-199

Stadt und Fest im kaiserzeitlichen Kleinasien (=Vestigia 39), München 1988

Lockender Lorbeer. Sport und Spiel in der Antike (=Ausstellungskatalog Staatliche Antikensammlung), München 2004

YALOURIS, N. (Hrsg.) The Olympic Games, Athen 1976

YAVETS, Z. Plebs and Princeps, Oxford 1969

YEGÜL, $F$. Bathing in the Roman World, Cambridge 2010

YOUNG, D. C. Professionalism in Archaic and Classical Greek Athletics, in: AW 7, 1983, 45-51

YOUNG, D. C., The Olympic Myth, Chicago 1985

YOUNG, D. C.
First with the Most: Greek Athletic Records and „Specialization", in: Nikephoros 9, 1996, 175-197 
ZANKER, P.

Die Barbaren, der Kaiser und die Arena. Bilder der Gewalt in der römischen Kunst, in: R. P. SIEFERLE/H. BREUNINGER (Hrsg.), Kulturen der Gewalt: Ritualisierung und Symbolisierung von Gewalt in der Geschichte, Frankfurt a. M./New York 1988, 53-86

ZEIDLER, H. Sport im römischen Köln, in: Nikephoros 5, 1992, 125-152

ZEVI, F. Des femmes-gladiateurs dans une inscription d'Ostie, in: MEFRA 88, 1976, 612-620

ZIEGLER, R. Städtisches Prestige und kaiserliche Politik. Studien zum Festwesen in Ostkilikien im 2. und 3. n. Chr. (Kultur \& Erkenntnis II), Düsseldorf 1985

ZIEHEN, L. RE 36 (1949), s. v. Panathenaia, 457-493

ZINGERLE, J . Der Steinwurf des Bybon, in: CV 2, 1936, 111-112 ZWIERLEIN-DIEHL, E. Antike Gemmen in deutschen Sammlungen, 2 Bde. (=Staatl. Mus. Preuß. Kulturbes. Antikensammlung Berlin), Berlin 1969 